\title{
LOW BIT-RATE IMAGE CODING FOR FACIAL MOVEMENT
}

\author{
'A Thesis Submitted \\ to the College of Graduate Studies and Research \\ in Partial Fulfillment of the Requirements \\ for the Degree of Master of Science \\ in the Department of Electrical Engineering \\ University of Saskatchewan \\ Saskatoon, Saskatchewan, Canada
}

by

Randal Todd Reinhardt

September 1997

(c) Copyright Randal Todd Reinhardt, 1997. All rights reserved. 


\section{PERMISSION TO USE}

In presenting this thesis in partial fulfillment of the requirements for a Postgraduate degree from the University of Saskatchewan, I agree that the Libraries of this University may make it freely available for inspection. I further agree that permission for copying of this thesis in any manner, in whole or in part, for scholarly purposes may be granted by the professor or professors who supervised my thesis work or, in their absence, by the Head of the Department or the Dean of the College in which my thesis work was done. It is understood that any copying or publication or use of this thesis or parts thereof for financial gain shall not be allowed without my written permission. It is also understood that due recognition shall be given to me and to the University of Saskatchewan in any scholarly use which may be made of any material in my thesis.

Request for permission to copy or to make any other use of material in this thesis in whole or in part should be addressed to:

Head of the Department of Electrical Engineering

57 Campus Drive

University of Saskatchewan

Saskatoon, Saskatchewan, Canada

S7N 5A9 


\section{ACKNOWLEDGMENTS}

This thesis could not have been written without the support and guidance by my supervisor Dr. Kunio Takaya. His experience and insight in this field helped develop a complete body of work.

I would like to thank TRLabs in Saskatoon for their financial assistance, and use of their facilities. As well, my thanks to the staff and students at TRLabs for their support.

I thank my parents, Grant and Joan, for their patience, encouragement, and support in my studies. I would like to thank my brother Lee for his time and support. A special thanks to my uncle Paul, and aunt Marlene for their help. 


\title{
UNIVERSITY OF SASKATCHEWAN
}

Electrical Engineering Abstract 96A454

\section{LOW BIT-RATE IMAGE CODING FOR FACIAL MOVEMENT}

\author{
Student: R.Todd Reinhardt \\ Supervisor: Prof. K. Takaya \\ M.Sc. Thesis Submitted to the \\ College of Graduate Studies and Research \\ September, 1997
}

\begin{abstract}
It is well known that video information uses large bandwidths for real-time transmission as in television broadcasting. Solving video bandwidth problems has focused on the use of image compression based on redundancy removal. However, this type of image co mpression alone is not capable of reducing the bit-rate sufficiently to allow real-time video through low bandwidth channels such as telephone lines. This method presented in this thesis takes a different approach. By limiting the type of images to facial images, this method encodes face expressions into motion parameters for the purpose of low bit-rate transmission. Through these parameters, movement of the person can be reconstructed by using a simulated face. Data requirements per frame can be reduced dramatically by the method allowing the frame rate to increase to the minimum video rate standard of 30 frames per second.

The implementation of the coding method requires an encoder and decoder. Since video is given as a sequence of images, a reference image is transmitted from the encoder to the decoder at the start. The encoder's operation is to extract and measure the fea tures of the face. This process uses edge detection, object extraction, and
\end{abstract}


object identification to find features. The extracted features are encoded with respect to the grid and the warping method used. Two methods of grid manipulating (action units and grid movement) and two method of warping (bilinear and cubic spline) are discussed in this thesis. Choice of method is based on operation speed, and algorithm complexity. The decoder was realized by the algorithms of the Attractor/Repellor for eyes, and mouth movement, 2-D motion algorithms for the head, and nonlinear scaling for head rotation.

The thesis demonstrated that the correct features can be found for 80 percent of the frames in a video clip database. It was also demonstrated that the decoder using the bilinear warping can successfully simulate eye and mouth movement as well as head motions including horizontal and vertical head turning and head rotation. 


\section{Table of Contents}

PERMISSION TO USE

ACKNOWLEDGMENTS

TABLE OF CONTENTS

LIST OF FIGURES

LIST OF TABLES

ABBREVIATIONS

1 Introduction

ii

$\mathbf{v}$

$\mathbf{x}$

xv

xvi

1

1.1 Motivation of the Research . . . . . . . . . . . . . 1

1.2 The Video Telephone Application ................ 2

1.3 Research Objective ....................... 4

1.4 Thesis Organization ................... 5

2 Facial Decoding by using Action Units 7

2.1 Introduction ........................ 7

2.2 The Model of the Face .................. 7

2.3 The Action Units . . . . . . . . . . . . . . 8

2.4 Interpretation of Node Movement . . . . . . . . . . . 11

2.4.1 Rotation .......................... 12

2.4 .2 Displacement ....................... 13

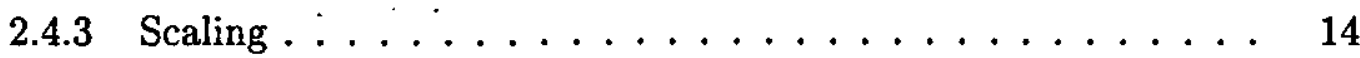


2.5 Facial Movement by the Action Unit . . . . . . . . . 15

2.6 Description of the Facial Features $\ldots \ldots \ldots \ldots \ldots$

2.7 Application of the Action Units to the Real Image . . . . . . . . 20

2.8 Evaluation of the Method $\ldots \ldots \ldots \ldots \ldots \ldots$

3 Warping Methods $\quad 25$

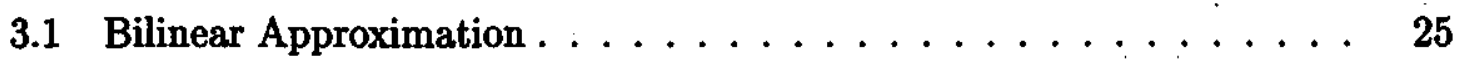

3.1.1 General Bilinear Approximation . . . . . . . . . . 25

3.1.2 New Bilinear Interpolation . . . . . . . . . . 30

3.1.3 Triangular Bilinear Interpolation using the New Algorithm . . 33

3.2 Cubic Spline Interpolation $\ldots \ldots \ldots \ldots \ldots \ldots$

3.2.1 Fant's Resampling Algorithm $\ldots \ldots \ldots \ldots \ldots$

3.3 The Application of the Warping Method . . . . . . . . . 40

4 Mesh Motion Control 41

4.1 Introduction . . . . . . . . . . . . . . 41

4.2 The Rectangular Mesh $\ldots \ldots \ldots \ldots \ldots \ldots$

4.2.1 Improved Grid Structure $\ldots \ldots \ldots \ldots \ldots \ldots$

4.3 Introduction to Mesh Movements . . . . . . . . . . 43

4.4 Attractor/Repellor Mesh Warping . . . . . . . . . . . . . 43

4.4.1 Theory of Operation $\ldots \ldots \ldots \ldots \ldots \ldots$

4.4 .2 Displacement of Grid Nodes . . . . . . . . . . . . 45

$4.5 \mathrm{X}-\mathrm{Y}$ Mesh Motion . . . . . . . . . . . . . . . . 48 
$4.5 .1 \quad X-Y$ Rotation $\ldots \ldots \ldots \ldots \ldots \ldots \ldots \ldots$

4.5.2 X-Y Displacement $\ldots \ldots \ldots \ldots \ldots \ldots \ldots \ldots$

4.5.3 X-Y Zooming (Linear Scaling) . . . . . . . . . . . . 49

4.6 Nonlinear Scaling . . . . . . . . . . . . . . . 50

5 Facial Feature Detection $\quad 54$

5.1 Digital Image Filtering $\ldots \ldots \ldots \ldots \ldots \ldots$

5.1 .1 How a Image Filter Works $\ldots \ldots \ldots \ldots \ldots \ldots$

5.1 .2 Edge Detection Filtering $\ldots \ldots \ldots \ldots \ldots$

5.1 .3 First Derivative Filters $\ldots \ldots \ldots \ldots 56$

5.1 .4 Orthogonal Gradient Generators $\ldots \ldots \ldots \ldots$

$5.1 .53 \times 3$ Gradient Generators . . . . . . . . . . . . 58

5.1.6 Edge Template Gradient Generation $\ldots \ldots \ldots \ldots$

5.1.7 2nd Order Derivative Filtering $\ldots \ldots \ldots \ldots \ldots$

5.1 .8 Laplacian Generation . . . . . . . . . . . . . . . 62

5.2 Facial Feature Extraction $\ldots \ldots \ldots \ldots \ldots$

5.2.1 Average Amplitude Projection . . . . . . . . . . 65

5.3 Directly Extractable Features $\ldots \ldots \ldots \ldots$. . . . . . 67

5.3.1 Application of Filters for Edge Detection . . . . . . . 67

5.3 .2 Finding the Objects $\ldots \ldots \ldots \ldots \ldots$

5.3 .3 Object Identification $\ldots \ldots \ldots \ldots \ldots \ldots$

5.4 Interframe Extractable Features $\ldots \ldots \ldots \ldots \ldots \ldots$ 


\section{List of Figures}

1.1 The Video Telephone System $\ldots \ldots \ldots \ldots \ldots$

2.1 The Base Mesh $\ldots \ldots \ldots \ldots \ldots \ldots \ldots$

2.2 The Action Unit 20 Muscles $\ldots \ldots \ldots \ldots \ldots$

2.3 The Simple Eye Grid $\ldots \ldots \ldots \ldots \ldots \ldots$

2.4 Rotation of a Node . . . . . . . . . . . . . . . . 12

2.5 Displacement of a Node $\ldots \ldots \ldots \ldots \ldots \ldots$

2.6 Scaling of a Node . . . . . . . . . . . . . . . . 14

2.7 (a) Reference and (b) Action Unit $20 \ldots \ldots \ldots \ldots$

2.8 The Mouth Grid $\ldots \ldots \ldots \ldots \ldots \ldots$

2.9 The Eye Grid $\ldots \ldots \ldots \ldots \ldots \ldots \ldots \ldots \ldots$

2.10 The Nose Grid $\ldots \ldots \ldots \ldots \ldots \ldots$

2.11 The Eyebrow Grid $\ldots \ldots \ldots \ldots \ldots \ldots$

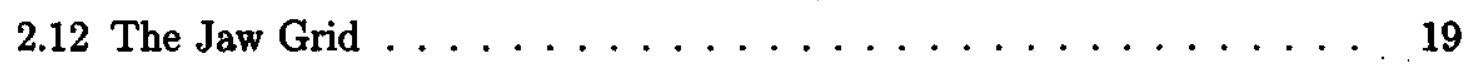

2.13 The Cheeks Grid . . . . . . . . . . . . . . . . . 19

2.14 (a)The Reference and (b)The Fitted Mesh . . . . . . . . . 20

2.15 Examples of mouth movements $\ldots \ldots \ldots \ldots 21$

2.16 Examples of Eye movements $\ldots \ldots \ldots \ldots \ldots$

2.17 Examples of Nose movements $\ldots \ldots \ldots \ldots 22$

2.18 Examples of Eyebrow movements . . . . . . . . . . . . 22 
5.3 The Gradient of a Edge $\ldots \ldots \ldots \ldots \ldots$

5.4 The Combination of the Orthogonal Gradients $\ldots \ldots \ldots \ldots 7$

5.5 The Prewitt Matrix $\ldots \ldots \ldots \ldots \ldots \ldots \ldots \ldots$

5.6 The Ideal Edge Detected Image $\ldots \ldots \ldots \ldots$

5.7 The Outline of the Head $\ldots \ldots \ldots \ldots 6$

5.8 First Sweep of the Face $\ldots \ldots \ldots \ldots \ldots \ldots$

5.9 The Combination of the Inner and Outer Sweeps to find the Objects 74

5.10 Scatter plot of Centroids from frames 0 to $9 \ldots \ldots 76$

5.11 A Typical Adaptive Head Split $\ldots \ldots \ldots \ldots$. . . . . . 78

5.12 Sweep Pattern for the Tilted Head $\ldots \ldots \ldots \ldots$

5.13 Relationship between $3-D$ and $2-D \ldots \ldots \ldots \ldots$

5.14 Example of Pattern Matching . . . . . . . . . . . 81

6.1 Test $1: 1$ Grid and 1 Mass Node . . . . . . . . . . . 84

6.2 Movement of Grid Nodes using $n$ and $\alpha \ldots \ldots \ldots \ldots$

6.3 Test 1: 2 Grid nodes and 1 Mass Node $\ldots \ldots \ldots \ldots \ldots$

6.4 Symmetrical Repellor action for 1 Mass Node $\ldots \ldots \ldots . \ldots 87$

6.5 Horizontal Symmetrical action for 1 Mass Node $\ldots \ldots \ldots$. . . . 87

6.6 Enhanced Horizontal Movement, Two Mass nodes . . . . . . . . 88

6.7 Enhanced Vertical Movement, Two Mass nodes $\ldots \ldots \ldots . \ldots 8$

6.8 Basic Position of Head Rotation Parameters . . . . . . . . . . . 89

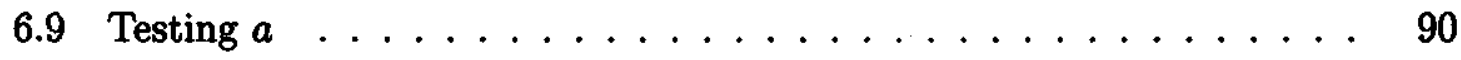


6.10 Nonlinear Motion of the Mesh . . . . . . . . . . . . . 91

6.11 Eye Movement for $n=1.5 \alpha=1.10 \ldots \ldots \ldots \ldots$. . . . . 93

6.12 Eye Movement for $n=2.0 \alpha=1.10 \ldots \ldots \ldots \ldots \ldots$

6.13 Eye Movement for $n=2.5 \alpha=1.10 \ldots \ldots \ldots \ldots \ldots$

6.14 Eye Movement for $n=1.5 \alpha=1.05 \ldots \ldots \ldots \ldots \ldots \ldots$

6.15 Eye Movement for $n=1.5 \alpha=1.075 \ldots \ldots \ldots \ldots \ldots 9$

6.16 Eye Movement for $n=1.5 \alpha=1.15 \ldots \ldots \ldots \ldots 9 . \ldots . \ldots 9$

6.17 Typical Eye Opening Action . . . . . . . . . . . . . 101

6.18 Typical Eye Closing Action $\ldots \ldots \ldots \ldots \ldots \ldots$

6.19 Mouth Movement for $n=1.5 \alpha=1.10$ and $\beta=0.50 \ldots \ldots 4$

6.20 Mouth Movement for $n=2 \alpha=1.10$ and $\beta=0.50 \ldots \ldots 105$

6.21 Mouth Movement for $n=2.5 \alpha=1.10$ and $\beta=0.50 \ldots \ldots 6$

6.22 Mouth Movement for $n=1.5 \alpha=1.013$ and $\beta=0.25 \ldots \ldots \ldots 108$

6.23 Mouth Movement for $n=1.5 \alpha=1.025$ and $\beta=0.37 \ldots \ldots \ldots 109$

6.24 Mouth Movement for $n=1.5 \alpha=1.05$ and $\beta=0.50 \ldots \ldots 110$

6.25 Lip Puckering . . . . . . . . . . . . . . . . . . 111

6.26 Typical Mouth Opening Movement . . . . . . . . . . . . 112

6.27 Typical Mouth Closing Movement . . . . . . . . . . . . . 113

6.28 X-Z Head Rotation Sequence $\ldots \ldots \ldots \ldots \ldots \ldots$

6.29 X-Z Head Rotation Grids $\ldots \ldots \ldots \ldots \ldots$

6.30 Y-Z Head Rotation Sequence $\ldots \ldots \ldots \ldots \ldots$ 
6.31 Y-Z Head Rotation Grids $\ldots \ldots \ldots \ldots$

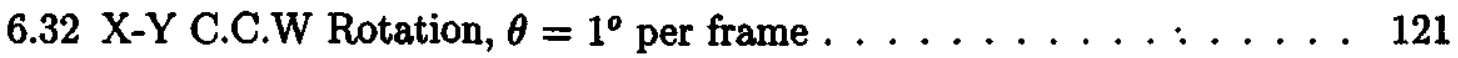

6.33 X-Y C.C.W Rotation Grids, $\theta=2^{\circ}$ per frame $\ldots \ldots \ldots \ldots$

6.34 X-Y C.W Rotation, $\theta=1^{\circ}$ per frame $\ldots \ldots \ldots \ldots \ldots$

6.35 X-Y C.W Rotation Grids, $\theta=2^{\circ}$ per frame $\ldots \ldots \ldots \ldots \ldots$

6.36 Tracking Eye Vertical Movement $\ldots \ldots \ldots \ldots$

6.37 Tracking Eye Horizontal Movement $\ldots \ldots \ldots \ldots$. . . . . . 127

6.38 Tracking of Mouth Movements . . . . . . . . . . . . 128

6.39 Width of the Head $\ldots \ldots \ldots \ldots \ldots \ldots \ldots$

6.40 Side of Head to Nose Widths . . . . . . . . . . . . . . 131

6.41 Variation of the distance between Nose and Mouth . . . . . . . 133

6.42 Variation of the distance between Nose to Middle of Two Eyes . . . . 134

6.43 Variation of the distance between Nose and Forehead $\ldots \ldots \ldots 135$

6.44 X-Y Rotation Measurements . . . . . . . . . . . . . . . 137

$6.45 \mathrm{X}-\mathrm{Y}$ Displacement (a) before and (b) after Rotation . . . . . . . 138 


\section{List of Tables}

2.1 Standard Action Units $\ldots \ldots \ldots \ldots \ldots \ldots \ldots \ldots$

3.1 Fant Algorithm Example . . . . . . . . . . . . . . . . 38

5.1 Robinson 5 -Level Filters $\ldots \ldots \ldots \ldots \ldots \ldots \ldots \ldots \ldots \ldots \ldots$ 


\section{ABBREVIATIONS}

$\mathrm{A} / \mathbf{R}$

CIF

CPU

1-D

2-D

3-D

DOS

DSP

fps

hz

IBM

ISDN

JPEG

kbaud

kbytes

Mhz

MPEG

NTSC

PC

QCIF attractor/repellor

common intermediate format

central processing unit

one dimensional

two dimensional

three dimensional

disk operating system

digital signal processor

frames per second

hertz ( $1 /$ second)

international business machines

integrated services digital network

joint photographic expert group

kilobits per second (1024 bits)

kilobytes (1024 bytes)

megahertz

moving pictures experts group

national television systems committee

personal computer

quarter common intermediate format 


\section{Introduction}

\subsection{Motivation of the Research}

The need for communicating with each other is a visual and audible experience. The demand for more personal contact with each other has prompted the need for realtime visual technology. This is the intent of the development of the video telephone. Due to limitations in technology, video telephones have not been accepted by the consumer.

Telephone lines are restricted to a bandwidth of $300-3400 \mathrm{hz}$. Therefore, video must be transmitted as digital information using existing modem technology. For digital video transmission three parameters must be considered, transmission rate of the modem, picture size, and picture quality. Current video telephones try to balance the three by using video image compression techniques.

Image compression is the ability to reduce data use in an image or group of images by taking advantage of the characteristics found in a particular image. There are many different types of image compression techniques, most methods quantize data, and reorganize data in the removal of redundant information. The choice of image compression must consider the CPU time spent on compression, and quality of the image. If much time is required in compression, or higher image quality is desired, then the speed of compression may not be able to keep up with the frame rate of motion pictures.

Technology in telephone line data transmission, and image compression cannot complete the goal of realistic video telephone transmission. What define realistic video transmission are fluidity of motion, and the synchronization of the mouth and words. The standard for image transmission in television broadcasting is set by the 
NTSC. This standard states that image transmission be 30 frames per second. Movie production uses a lower frame rate of $24 \mathrm{fps}$. As the frame rate reduces, the faster movements appear jittery. Currently, transmission of video over telephone lines is limited to 34 kbaud [1]. A common compression method such as JPEG can easily achieve a compression ratio of $20: 1$. This means that for a full $30 \mathrm{fps}$ under these conditions the average frame rate allows only 2.8 Kbytes of uncompressed data per frame. To achieve NTSC picture size ${ }^{1}$ (at 8 bits per pixel) the compression ratio must be as high as 600:1. Also, this high compression gain of 600:1 must be accomplished in one thirtieth of a second. If the picture size is reduced to the smallest standard $\mathrm{QCIF}^{2}$ the compression ratio must be $180: 1$, however resolution is reduced. Such very large compression ratios are difficult to achieve. Compression of a single image using the advanced method wavelets [2] at best is 150:1.

\subsection{The Video Telephone Application}

The facial coding scheme described in this thesis has a application to the video telephone. The encoding section acts as the transmitter of the telephone, and the decoding corresponds to the receiver. The structure of such a system is shown in Fig. 1.1. Very condensed information conveying facial parameters is sent from the receiver to the transmitter in real-time at a very low bit-rate.

The main purpose of the video telephone is to see a person, or more specifically the person's face. The face can be broken down into regions such as the eyes, nose, mouth, and head. Since the action of each feature can be measured and described in symbolic terms, it is possible to reduce the information pertaining to facial expressions drastically. If the face of the talker is once sent, a face having certain facial expressions can be reconstructed with the help of the condensed symbolic information.

The system requires an image as a reference for all motions that occurs in the person's face. All video received after the reference image are artificially reconstructed

\footnotetext{
${ }^{1}$ picture size is 352 by 240 pixels

${ }^{2}$ picture size is 176 by 144 pixels
} 


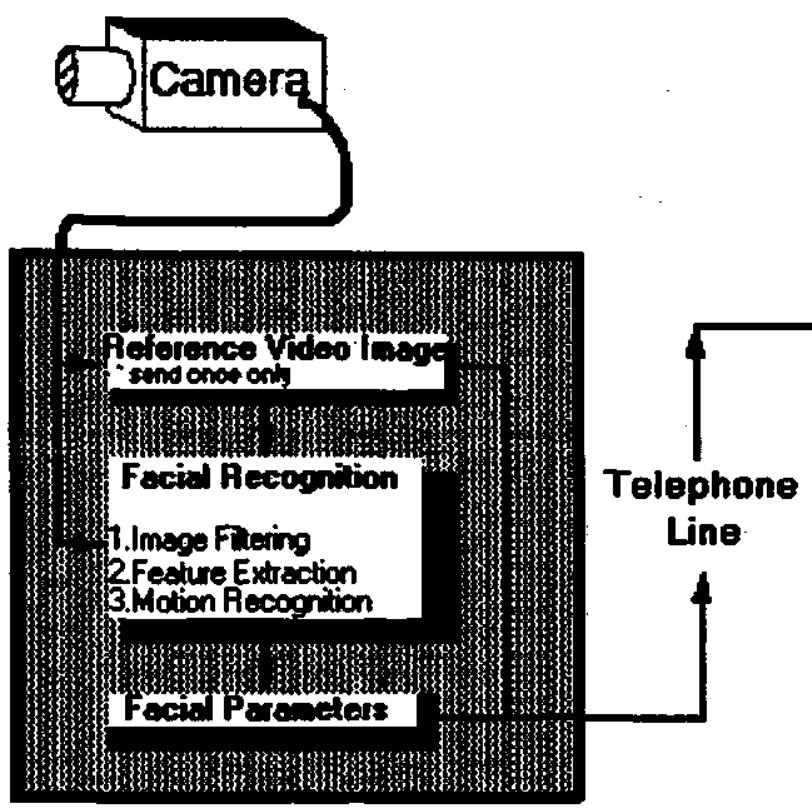

Transmitter

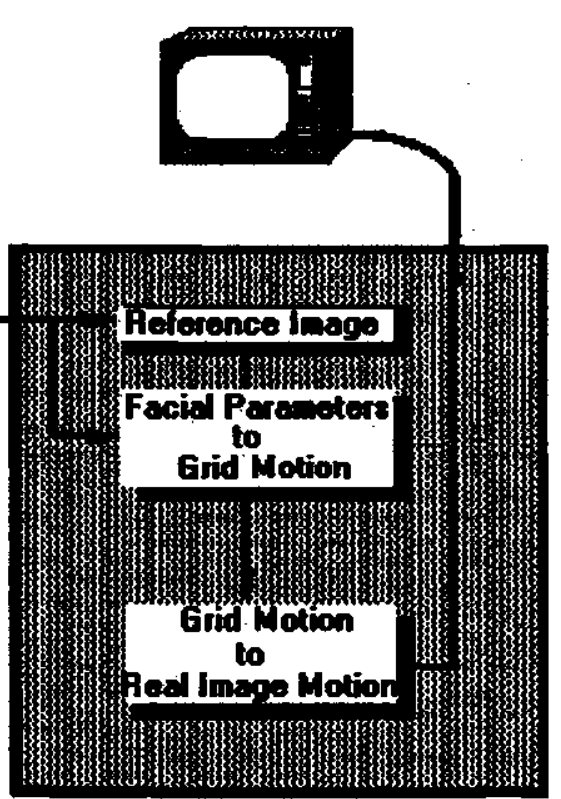

Receiver

Figure 1.1 The Video Telephone System

by warping the reference image. A mesh that fits the face determines how the face should be warped. Facial recognition use various methods such as image filtering, feature extraction, and motion detection to calculate mesh grid deformation parameters that realize a facial expression.

The reference image and initial grid information must be transmitted to the receiver upon start up. Thereafter, such parameters detected by the transmitter are sent to the receiver within one frame time. The receiver then use the parameters to manipulate and warp the reference according to mesh grid movement 


\subsection{Research Objective}

Research objectives of the thesis project is to investigate and the design a low bitrate video encoding system for video telephone and video conferencing applications using low bit-rate channels such as telephone lines. The image dealt in this research is therefore limited to facial video images. Such coding systems are comprised of a encoder(transmitter) and decoder(receiver). Expected system requirements and individual requirements for the major components, encoder and decoder are summarized in what follows.

Low bit-rate coding is a coding scheme with the purpose of reducing a sequence of image data of a person into measurement parameters, then reconstruct the image with the parameters. The requirement of the image data is that each frame contains all face and head features. From this image, data should be definable in terms of the eyes, mouth, and head components. The coded data must translate its action with respect to a reference image of the same person using a grid. Coded information should be small enough to send up to 30 coded images per second over low data rate channels such as $300-3400 \mathrm{hz}$ telephone line. Achieving a real-time video system is based on the ability to reach the minimum frame rate. Justification of low bit-rate transmission will be based on coded data size rather than implementation of a real communications link.

The encoder must be able to define facial parameters such as the eyes, mouth, and head from the image. Successful extraction at the levels of edge detection, object extraction, and object identification are a requirement in order to find the parameters. Eyes and mouth features are expected to the most difficult to find. Measurements of these features should be able to show the opening and closing operations. In head detection, movement is expected in all three dimensions. Testing should be performed to determine what features of the face could be used.

The decoder is responsible for simulating movement through a defined grid. All algorithms must translate features to the same grid before the image can be manipu- 
lated. Again, each method relates to the eyes, mouth, and head. A successful decoder is expected to reproduce the actions of head rotation, and eye and mouth movement using a reference image. The decoding action must be restricted to a limited data set, and relate to the encoder extraction methods. Speed is the primary concern in the choice of warping algorithm, however choice will be based on algorithm complexity.

\subsection{Thesis Organization}

The thesis is organized into 7 chapters.

Chapter 1 provides background materials and motivation for the thesis, the objectives of this research are also stated.

Preliminary work on a high level method of facial coding called Action Units is covered in chapter 2. This work aids in the understanding for psycho-physiological facial coding by categorizing each facial movement on muscle movement.

Chapter 3 covers the theory behind the facial decoding used. This chapter specifically discusses the techniques related to image warping. Image warping covers the two common methods Bilinear, and Cubic Spline interpolation. The bilinear method fits image data to a polygon mesh. The two types of polygons covered are rectangular (used in chapter 4) and triangular (used in chapter 2). Cubic Spline interpolation is a complex method that moves pixels to a smooth curve passing through the mesh points of a warped image.

Chapter 4 covers general techniques of moving a mesh and their applications for the movement of eyes and mouth in a 2-D space X-Y, and head movement in a 3-D space X-Y-Z. The eyes and mouth are controlled by the algorithm called the Attractor/Repellor. X-Y head rotation is controlled by standard methods of two dimensional image displacement, rotation, and scaling. $\mathrm{X}-\mathrm{Z}$ and $\mathrm{Y}-\mathrm{Z}$ head rotation is controlled by a nonlinear function for manipulating the grid. The basis of all these methods is to use a rectangular grid.

Encoding of the face is covered in chapter 5. This involves algorithms for extrac- 
tion of the main features in each frame. As well frame to frame features such as head rotation are discussed. The encoder discussed in this chapter is a basic encoder in the developmental stage. The ideas expressed can be extended to a more complete encoder.

Chapter 6 presents the results of the algorithms discussed in chapters 3,4 , and 5 .

Chapter 7 summarizes the research preformed and the results. In addition, future work is suggested for improvement of the performance of the low bit-rate encoding. 


\section{Facial Decoding by using Action Units}

\subsection{Introduction}

The chapter covers the work completed in the early portion of the project. The focus of this work is to use predefined motions of the face called action units to encode and decode the face. Motion of the face is accomplished by a two-dimensional mesh representation of the face, or a mask overlaying the real image. The mask divides the face into triangular sections, which form a collection of the simplest polygons. Action units are the parameters that translate a facial expression to mask deformation, and in turn warp the overlaid image to give the appearance associated with facial expression.

\subsection{The Model of the Face}

The design of the grid was to achieve a mesh that closely resembles a face. The face model is a set of interconnected triangles that approximate the face. The initial base grid defines eyes, mouth, and nose but do not correspond to any known face. The base grid shown in Fig. 2.1 is made of 278 nodes and 280 triangles. The nodes that cover the eyes, mouth, and head boundary are the most important to simulating motion. The eyebrows, nose, cheek, and jaw are included in the base mesh to make it complete. 


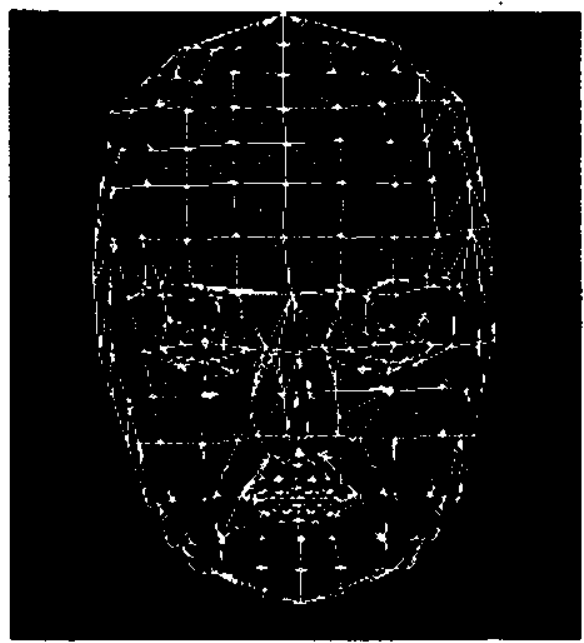

Figure 2.1 The Base Mesh

\subsection{The Action Units}

The premise of describing facial movements was done by Paul Ekman and W.V Friesen, who work in the field of Psychology. Their work initially started in the mid 1970's with the culmination of their work being published in Ekman [3] as a manual for recognizing facial expressions. The manual identifies 46 different "Action Units" of the face. The description of an action unit is based on muscles in the face, where muscles are labeled with particular action numbers. For example, if the mouth action is the "Lip Stretcher" then the muscles associated with as action unit 20 in Fig. 2.2 must have moved. These action units are carefully categorized by visual observation of facial movements. Action units according to the manual are defined by four main groups, (1) Appearance change, (2) How to do action, (3) Minimum requirements for action, and (4) Intensity Scoring for action. 


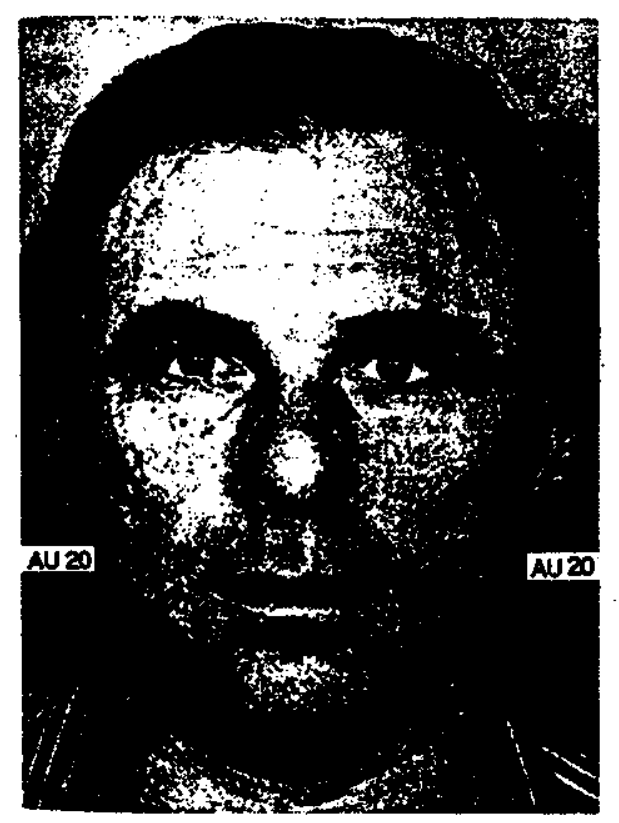

Figure 2.2 The Action Unit 20 Muscles

The adoption of action units to computer simulations was done by Choi et al. [4]. In this article, the action units are implemented in a 3-D facial model. Of the 46 action units 34 were chosen for testing. These action units are listed in Table 2.1. Action units can be applied alone or in combination with others to produce the desired facial expression. The preliminary thesis work was to use a similar method to Choi et al. [4], but only describe the actions in terms of 2-D motion. 
Table 2.1 Standard Action Units

\begin{tabular}{|l|l|l|l|}
\hline No. & Action Unit & No. & Action Unit \\
\hline 1 & Inner Brow Raiser & 20 & Lip Stretcher \\
2 & Outer Brow Raiser & 23 & Lip Tightener \\
4 & Brow Lowerer & 24 & Lip Pressor \\
5 & Upper Lid Raiser & 25 & Lips Part \\
6 & Cheek Raiser & 26 & Jaw Drop \\
7 & Lid Tightener & 27 & Mouth Stretch \\
8 & Lips Toward & 28 & Lips Suck \\
9 & Nose Wrinkler & 29 & Jaw Thrust \\
10 & Upper Lip Raiser & 30 & Jaw Sideways \\
11 & Nasolabial Furrow & 32 & Bite \\
& Deepener & 35 & Cheek Suck \\
12 & Lip Corner Puller & 41 & Lid Droop \\
13 & Sharp Lip Puller & 42 & Slit \\
14 & Dimpler & 43 & Eyes Closed \\
15 & Lip Corner Depressor & 44 & Squint \\
16 & Lower Lip Depressor & 45 & Blink \\
17 & Chin Raiser & 46 & Wink \\
18 & Lip Pucker & & \\
\hline
\end{tabular}




\subsection{Interpretation of Node Movement}

If all the action units described in Table 2.1 are to be implemented then the complexity of the face grid is essential. To move a facial part say the mouth, certain nodes are more important than others these points are called control nodes (see Fig. 2.3). For example, in the opening and closing of the eye two likely control nodes are at the top and bottom of the eye as shown in Fig. 2.3. If the eye in a reference frame was open and the eye in the following frame was closing (Action Unit 43), then a ratio of the distance $W$ can be determined between two frames. This ratio is referred to as the intensity of the action. The other points that make up the eye are called secondary nodes (Fig. 2.3), and move with respect to the control points. In the eye example, the secondary nodes will move in proportion with the control nodes to achieve the opening and closing of the eye.

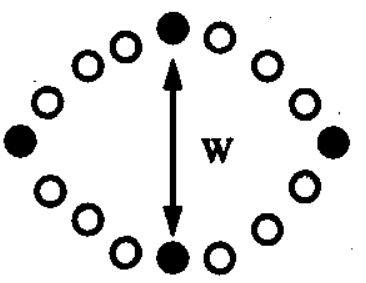

Control Node

O Secondary Node

Figure 2.3 The Simple Eye Grid

Movements of nodes can be calculated using three standard methods of vector motion, namely displacement, rotation, and scaling. 


\subsubsection{Rotation}

Rotation is the angular movement of nodes with respect to a fixed node. A fixed node $P_{1}$ must be set for each facial part, but it is not necessary to coincide with facial nodes. The rotation of a node is calculated by Eq. 2.1. The formula rotates a vector $P_{2}-P_{1}$ to produce a new vector $P_{2}^{\prime}-P_{1}$.

$$
\left[\begin{array}{c}
Y_{12}^{\prime} \\
X_{12}^{\prime}
\end{array}\right]=\left[\begin{array}{rr}
\cos (\theta) & \sin (\theta) \\
-\sin (\theta) & \cos (\theta)
\end{array}\right]\left[\begin{array}{c}
Y_{12} \\
X_{12}
\end{array}\right]
$$

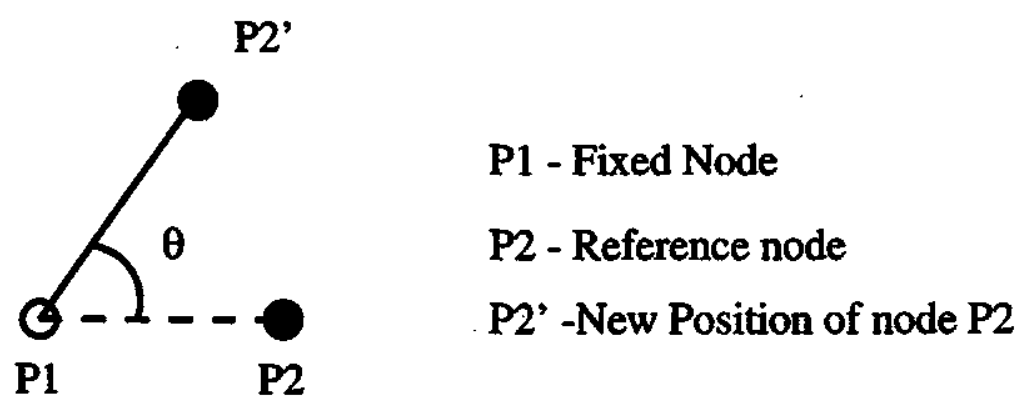

Figure 2.4 Rotation of a Node 


\subsubsection{Displacement}

The movement is simple addition of a constant to each of two coordinates. The formula Eq. 2.2 shows the calculation required. The amount of displacement in the $\mathrm{y}$ and $\mathrm{x}$ direction is specified by $a$ and $b$ (as shown in Fig. 2.5). Point $P_{2}$ is thus displaced to point $P_{2}^{\prime}$ with respect to the reference node $P_{1}$.

$$
\left[\begin{array}{c}
Y_{12}^{\prime} \\
X_{12}^{\prime}
\end{array}\right]=\left[\begin{array}{l}
Y_{12} \\
X_{12}
\end{array}\right]+\left[\begin{array}{l}
a \\
b
\end{array}\right]
$$

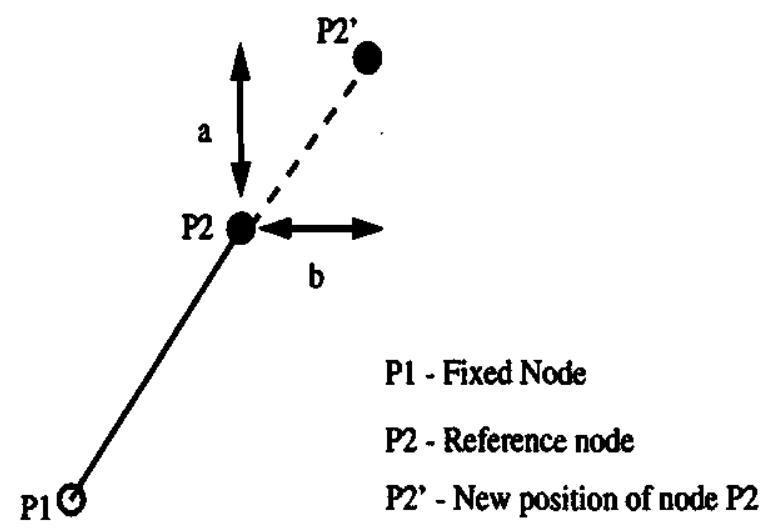

Figure 2.5 Displacement of a Node 


\subsubsection{Scaling}

This is a special case of displacement, which changes the length of a vector but not the direction. In Fig. 2.6, if the original length is one then the vector is extended by the ratio 1:a. Formula Eq. 2.3 shows the use of one scale factor applied to both $\mathrm{x}$ and y coordinates.

$$
\left[\begin{array}{c}
Y_{12}^{\prime} \\
X_{12}^{\prime}
\end{array}\right]=\left[\begin{array}{c}
Y_{12} * a \\
X_{12} * a
\end{array}\right]
$$

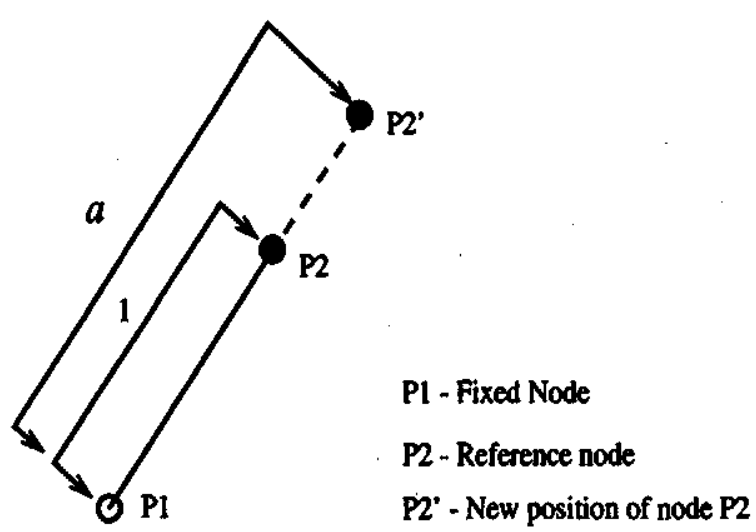

Figure 2.6 Scaling of a Node 


\subsection{Facial Movement by the Action Unit}

Each action unit must be designed for a specific part of the face. The action unit contains information on mask nodes, fixed nodes, and movement of each node with respect to rotation, displacement, and scaling. The process of using of action units are best described by showing an example. Fig. 2.7 (a) is the reference mesh of the entire face, and all action units in Table 2.1 affect this mesh. If a person had spoken the letter " $\mathrm{E}$ " then the program will make a decision based on the measured control points. Of the action units available it may have decided that action unit 20, "Lip Stretcher", was the best fit. The choice of "Lips Stretcher" was deduced from the comparison of all action units and combination of action units. The intensity of the action is found by determining the amount of change in the control nodes. An action unit decoder would receive the action unit information to reproduce the motion. The result is shown in Fig. 2.7(b).

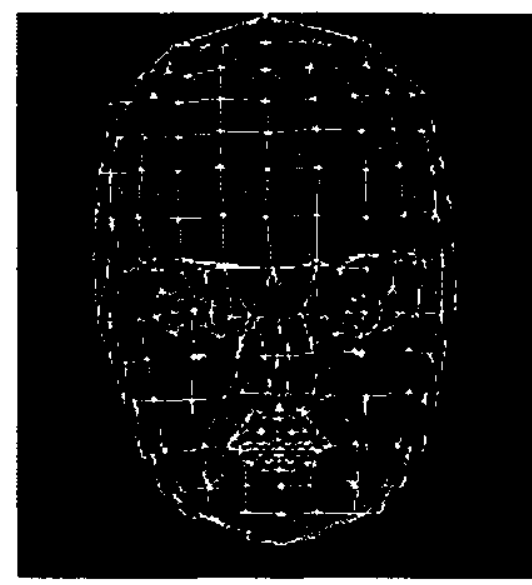

(a)

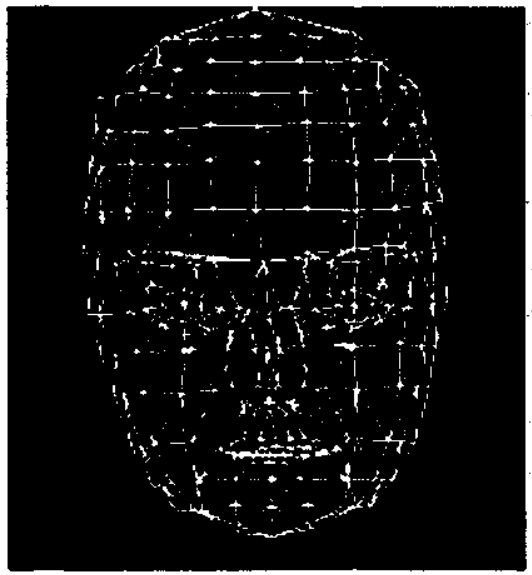

(b)

Figure 2.7 (a) Reference and (b) Action Unit 20

The action unit of the "Lip Stretcher" is mainly vector displacement. Only 24 out of the 39 mouth nodes are moved using one fixed node. The position of the fixed node is at the center of the mouth. 


\subsection{Description of the Facial Features}

The program developed can control six features by action units. These features involve action units associated with the eyes, eyebrows, mouth, nose, cheeks, and jaw. The interpretation of the action units was done by personal observation deciding on how the nodes should move to achieve the best-simulated action. The base grid in Fig. 2.1 was found satisfactory to describe these action units. 
The Mouth

Characteristics:

Nodes: 39

Action Units : 11

Fixed Nodes : 2

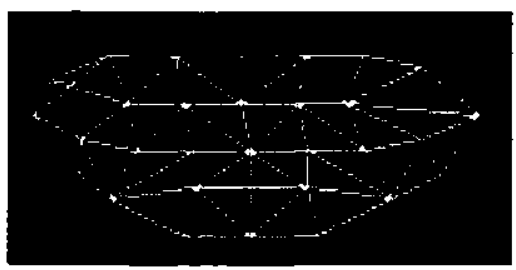

Figure 2.8 The Mouth Grid

Important Features:

Has a wide range of movement in all directions.

Applying certain action units can allow a mouth to open revealing the inside of the mouth.

\section{The Eyes}

Characteristics:

Nodes: 24 (12 per eye)

Action Units : 7

Fixed Nodes : 2 ( 1 per eye)

Important Features:

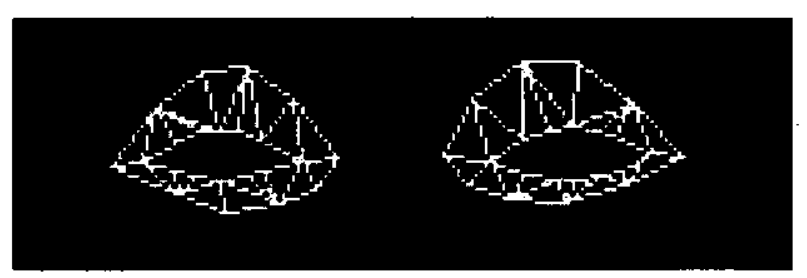

Figure 2.9 The Eye Grid

The eye's action units may be applied for one eye or both.

Nodes, which apply are at the perimeter of the eye.

The eye has limited horizontal movement.

Properly closing the eyes with this method is difficult . 


\section{The Nose}

Characteristics:

Nodes: 11

Action Units : 2

Fixed Nodes : 1

Important Features:

The nose has limited movement, therefore only two action units are required.

\section{The Eyebrows}

Characteristics:

Nodes: 18 (9 per eyebrow)

Action Units : 3

Fixed Nodes : 2 (1 per eyebrow)

Important Features:

The eyebrows action units apply for one or both.

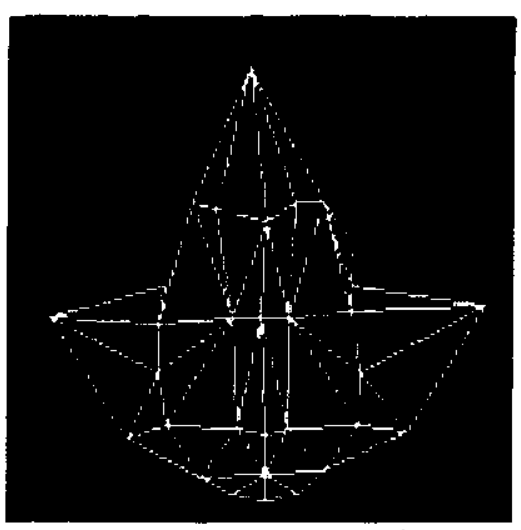

Figure 2.10 The Nose Grid

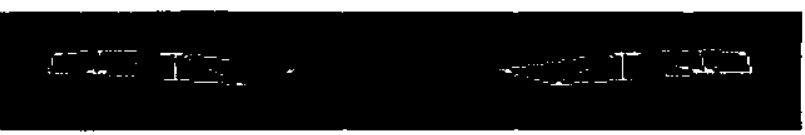

Figure 2.11 The Eyebrow Grid 


\section{The Jaw}

Characteristics:

Nodes: 52

Action Units : 3

Fixed Nodes : 1

Important Features:

The circular shape of the jaw makes it ideal for using vector scaling.

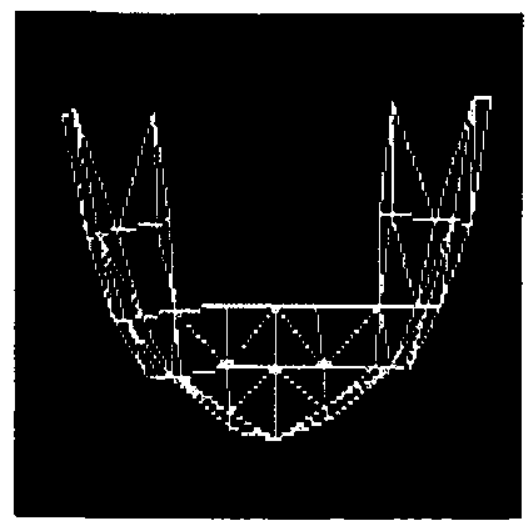

Figure 2.12 The Jaw Grid

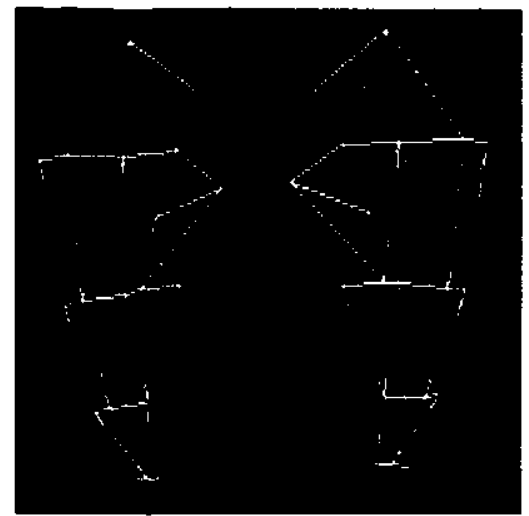

Figure 2.13 The Cheeks Grid 


\subsection{Application of the Action Units to the Real Image}

The base grid is set as a reference to describe how the subsequent action should take place. A complete program was developed to incorporate the algorithms for decoding a real face. The choice of triangles as sections allows the grid to fit to any object. With this property, action units are translated through the grid without any restrictions. To complete the decoder bilinear interpolation for triangles (detailed in chapter 3) provides the image warping.

The fitting of the image of the face to the mesh requires the location and size of the facial parts of a person. To relocate the features in the base grid to fit a given face, the triangles need to be compressed or expanded. For the testing of the action units Fig. 2.14(a),the image of "Todd", is the source image. To fit the face to the mesh information on the mouth, eyes, nose, and head is required. The other parts of the face are placed with respect to the known information. The mesh of the image of "Todd" is shown in Fig. 2.14(b). With a properly fitted image the application of the action units can be done.

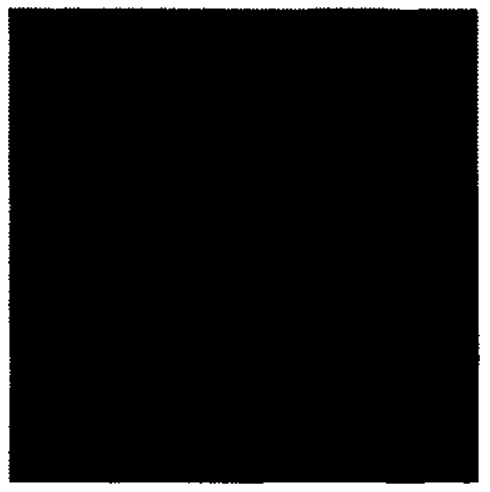

(a)

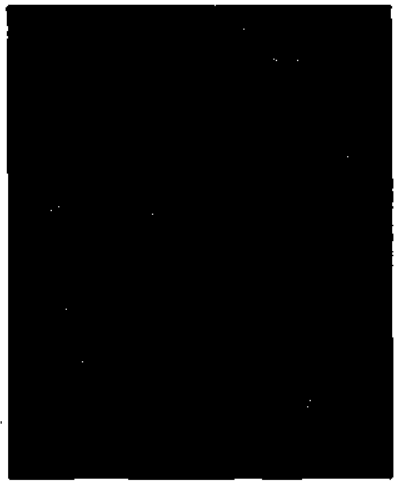

(b)

Figure 2.14 (a)The Reference and (b)The Fitted Mesh

The following examples of each facial feature in motion illustrate the results obtained by using Fig. 2.14(b) as the reference. 

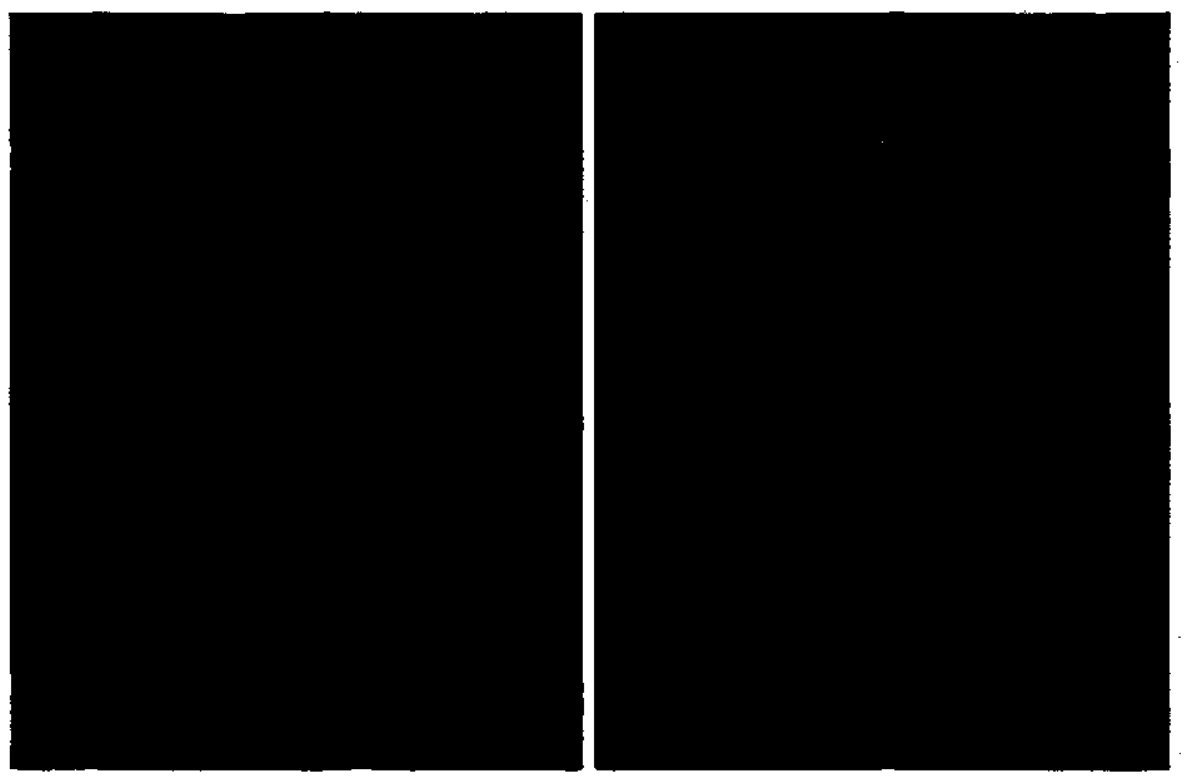

Figure 2.15 Examples of mouth movements Action Unit 25 "Lips Part" and Action Unit 24 "Lips Pressor".
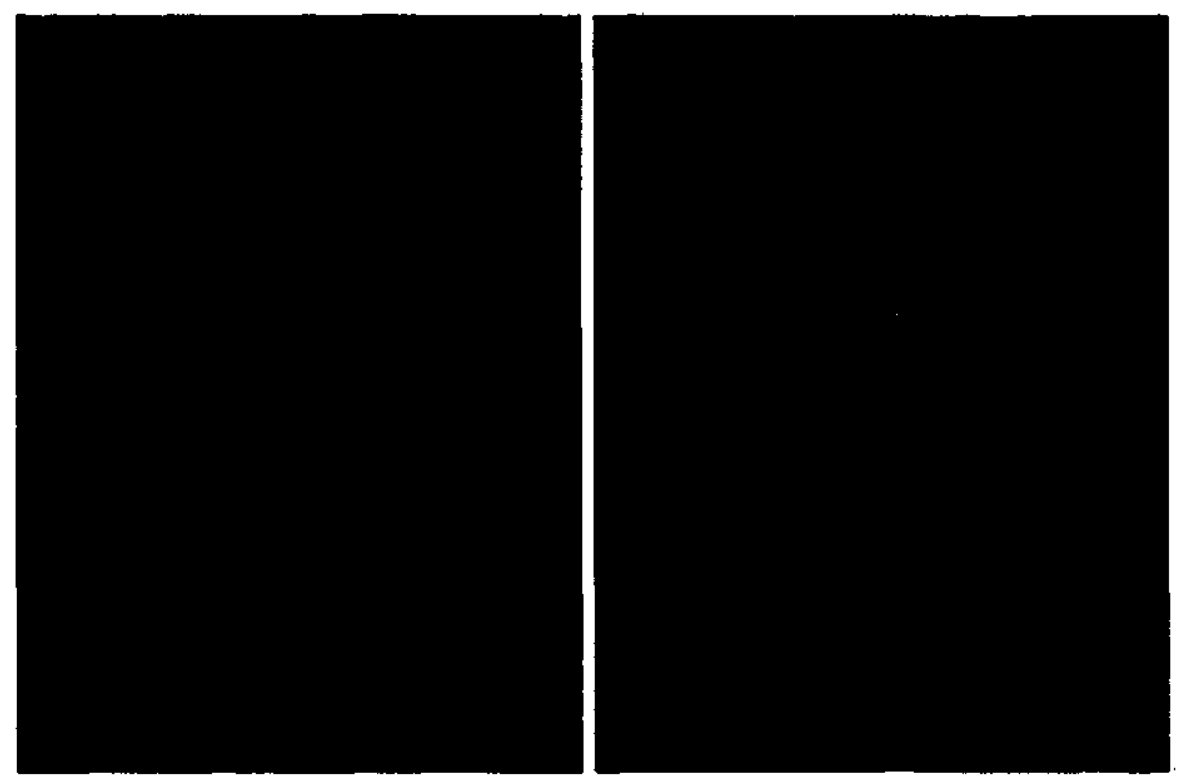

Figure 2.16 Examples of Eye movements

Action Unit 43 "Eyes close" and Action Unit 43 applied in the opposite direction. 


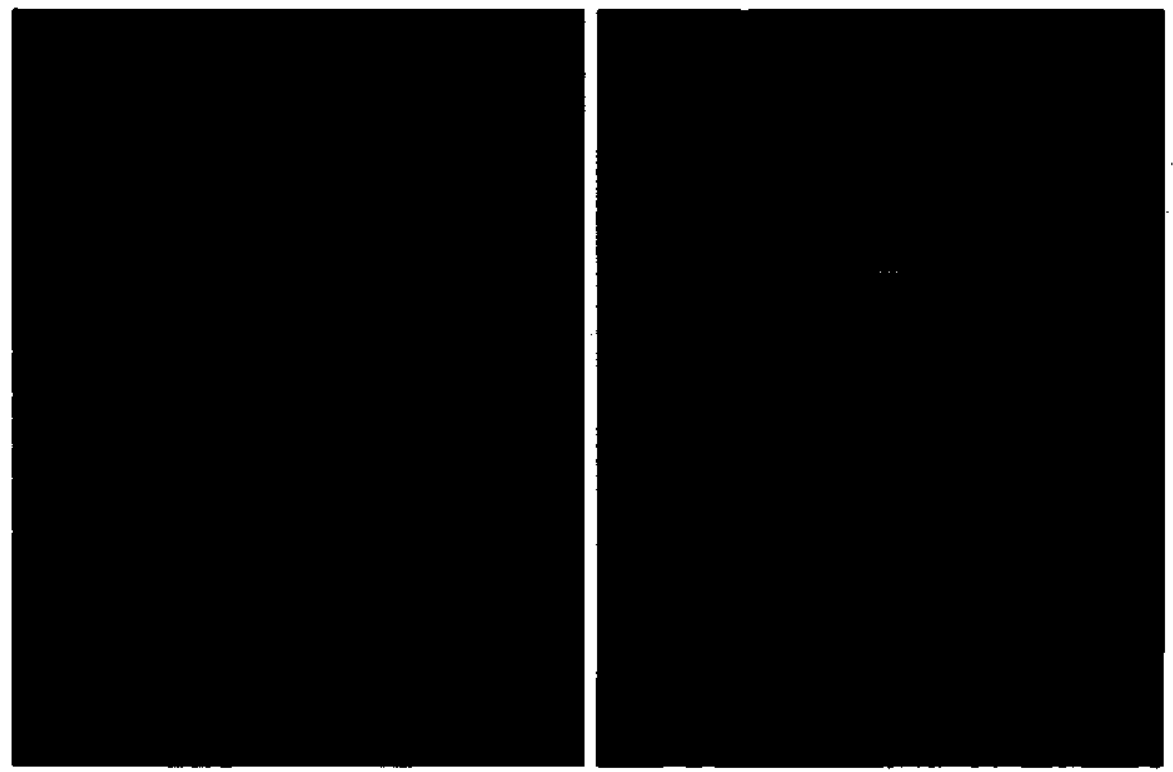

Figure 2.17 Examples of Nose movements

Action Unit 11 "Nasolabial Furrow Deepener" and Action Unit 9 "Nose Wrinkler".
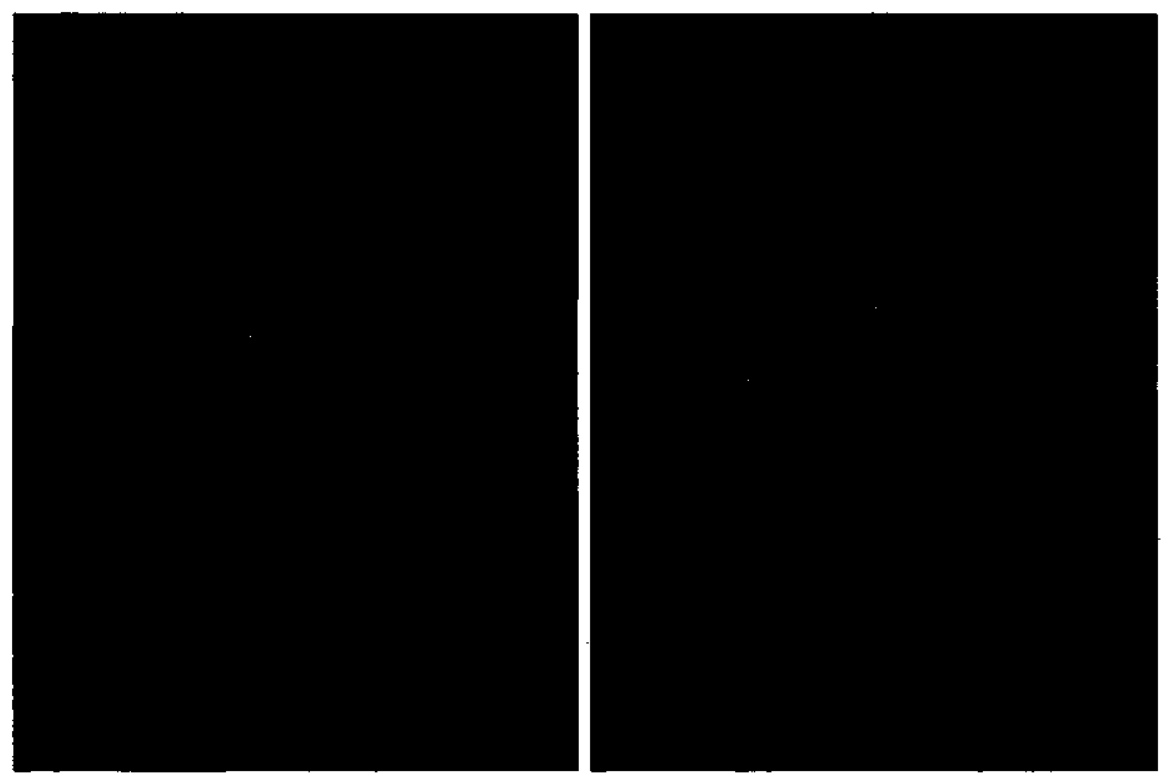

Figure 2.18 Examples of Eyebrow movements Action Unit 4 "Brow Lower" and Action Unit 2 "Outer Brow Raiser". 


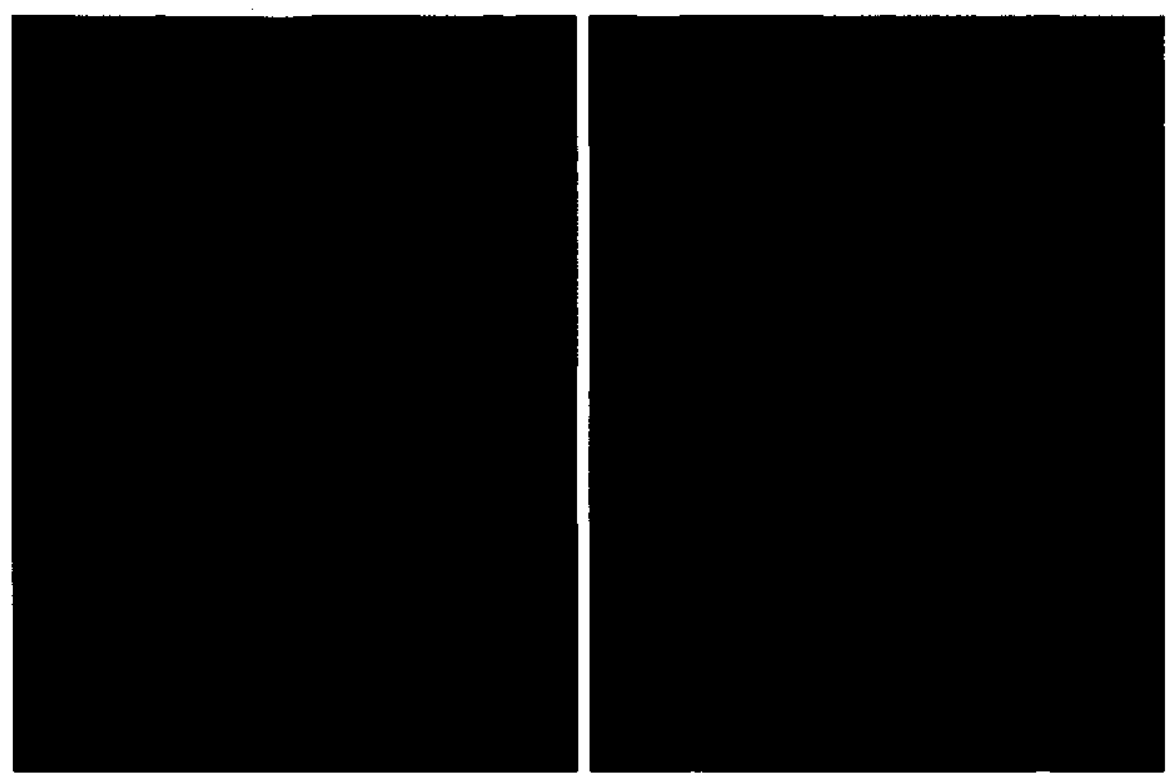

Figure 2.19 Examples of Jaw movements

Action Unit 29 "Jaw Thrust" and Action Unit 30 "Jaw Sideways".

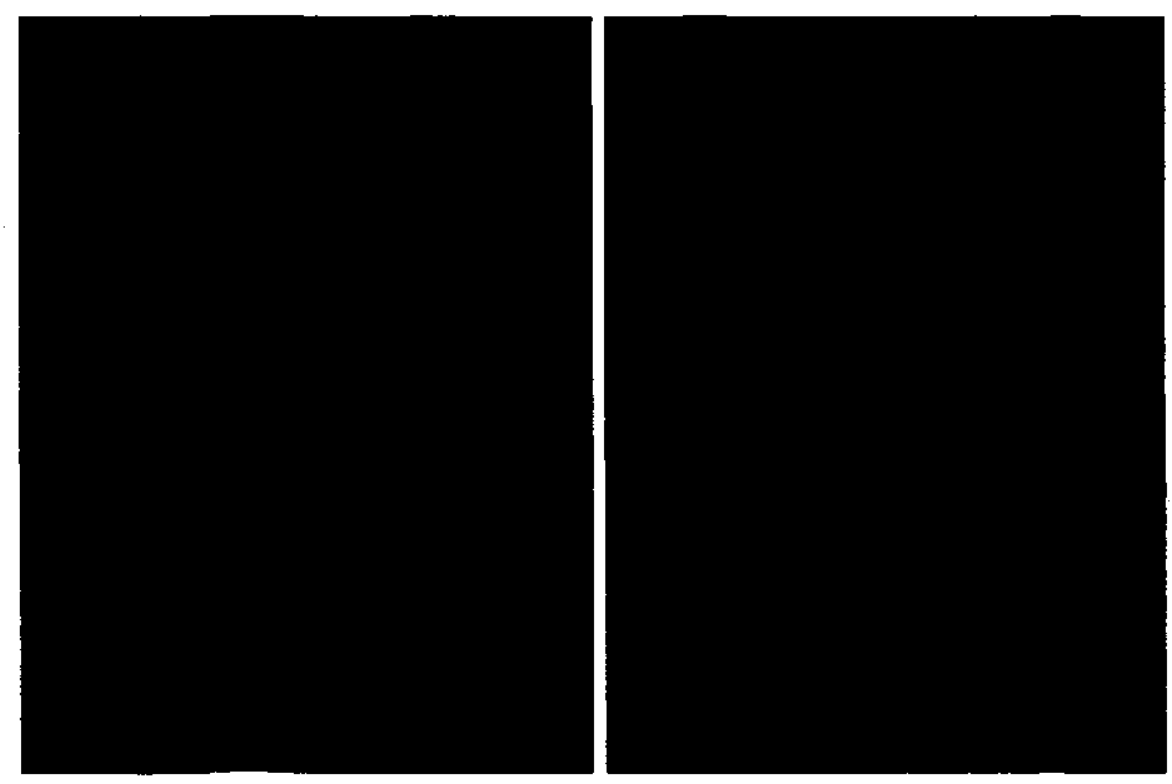

Figure 2.20 Examples of Cheek movements Action Unit 35 "Check Suck" and Action Unit 6 "Cheek Raiser". 


\subsection{Evaluation of the Method}

The use of action units and triangular grids is a valid method in coding of a human face. The triangular grids can produce a realistic simulated facial expression and easily adopt the action units. The grid is flexible enough to realize six facial features with the help of the action units. Action units are in fact a database of facial movements. The action units are applicable to most faces once the base grid has been fitted to a face. Once initial setup is complete, the system can produce simulated facial images using the image-warping algorithm.

This method was chosen as a preliminaries study because action units classify facial movements into distinct categories. All movements have been divided into 34 action units, which also can be combined to give a wide range of facial expressions. However, the development of a more practical method is inevitable because the method requires interactive grid setting, and cannot be automated. When the pictures are too small, not enough detail can be extracted to distinguish the action units. If the pictures are too large then the time required on encoding and decoding increases by the length to a power of two.

If the encoder design is to choose action units, a smart algorithm is necessary. This can be very complex and slow if action units are combined because each action may affect the same points. The computer must therefore be programmed to decide which of the action units to use and determine how action units should be combined. With a fast picture frame rate, such as $\mathbf{3 0}$ frames per second, the amount of movement from frame to frame may be very little (image size is also a factor in this). Small movements make selection of proper action units more difficult.

From these difficulties, the development of a simpler mesh was inevitable. A rectangular mesh will be introduced in chapter 4 . This preliminary work is valid, but it is concluded that the work cannot meet the objectives of real-time facial coding. 


\section{Warping Methods}

In order to present a facial expression, the mesh, the canvas in another word, must be effectively painted with an image of a face. This chapter discusses the standard methods of warping. Warping is the deformation of a source image by means of a destination grid. The nodes in the grids are reference points for deformation. The theoretical background of the image deformation is discussed in relation to the grid movement.

There is two basic methods of warping, bilinear interpolation and cubic spline interpolation. Bilinear interpolation is a simpler method, but it has drawbacks in image quality. However, the cubic spline method is just the opposite with a more complicated algorithm and higher quality. It will be discussed at the end of this chapter what method is more suited for the project.

\subsection{Bilinear Approximation}

This section first focuses on the general method of bilinear interpolation, then it discusses two bilinear interpolation methods which are specific to this project. These methods of bilinear interpolation are formulated for the deformation of a rectangular block, and a triangular block.

\subsubsection{General Bilinear Approximation}

When a surface is defined by

$$
U(x, y)=c_{1}+c_{2} x+c_{3} y+c_{4} x y
$$




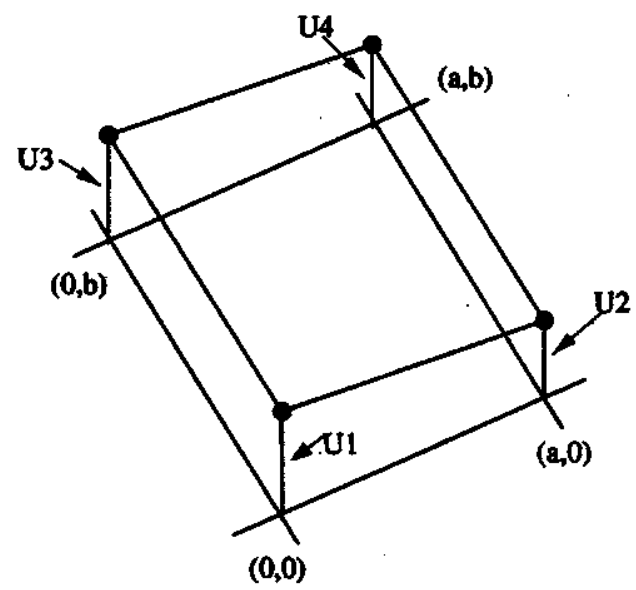

(a)

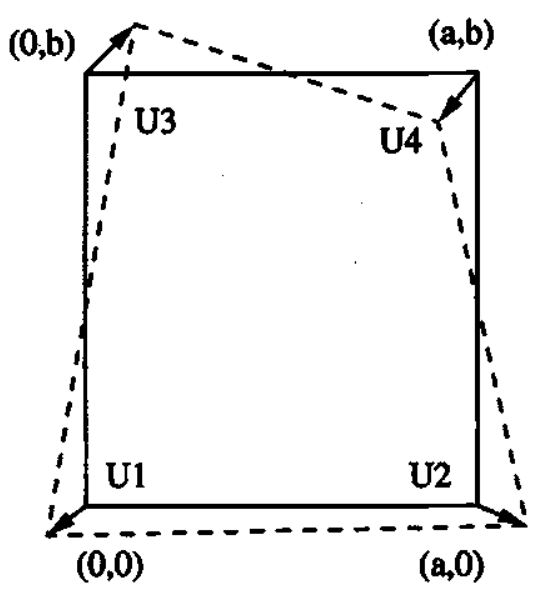

(b)

Figure 3.1 General Bilinear Interpolation

We assume that the surface passes through four points $U_{1}, U_{2}, U_{3}$, and $U_{4}$ as shown in Fig. 3.1 (a).

The variables $(x, y)$ represent an arbitrary point in the rectangle. The four points $(0,0),(a, 0),(0, b)$, and $(a, b)$ are the coordinate values for $U_{1}, U_{2}, U_{3}$, and $U_{4}$ respectively. Therefore, each of these four points can be expressed in terms of the coefficients $c_{1}, c_{2}, c_{3}$, and $c_{4}$ of Eq. 3.1 as follows

$$
\begin{gathered}
U_{1}=U(0,0)=c_{1} \\
U_{2}=U(a, 0)=c_{1}+c_{2} a \\
U_{3}=U(0, b)=c_{1}+c_{3} b \\
U_{4}=U(a, b)=c_{1}+c_{2} a+c_{3} b+c_{4} a b
\end{gathered}
$$


Solving Eq. 3.2 through Eq. 3.5, we obtain the unknown coefficients $c_{1}, c_{2}, c_{3}$, and $c_{4}$.

$$
\begin{gathered}
c_{1}=U_{1} \\
c_{2}=\frac{U_{2}-U_{1}}{a} \\
c_{3}=\frac{U_{4}-U_{1}}{b} \\
c_{4}=\frac{U_{3}-U_{2}-U_{4}+U_{1}}{a b}
\end{gathered}
$$

Eq. 3.6, 3.7, 3.8, and 3.9 can be shown as vector equation

$$
\left[\begin{array}{c}
c_{1} \\
c_{2} \\
c_{3} \\
c_{4}
\end{array}\right]=\left[\begin{array}{cccc}
1 & 0 & 0 & 0 \\
1 & a & 0 & 0 \\
1 & a & b & a b \\
1 & 0 & b & 0
\end{array}\right]^{-1}\left[\begin{array}{c}
U_{1} \\
U_{2} \\
U_{3} \\
U_{4}
\end{array}\right]
$$

Using the inverse matrix, the equation becomes,

$$
\left[\begin{array}{c}
c_{1} \\
c_{2} \\
c_{3} \\
c_{4}
\end{array}\right]=\frac{1}{a b}\left[\begin{array}{rrrr}
a b & 0 & 0 & 0 \\
-b & b & 0 & 0 \\
-a & 0 & 0 & a \\
1 & -1 & 1 & -1
\end{array}\right]\left[\begin{array}{c}
U_{1} \\
U_{2} \\
U_{3} \\
U_{4}
\end{array}\right]
$$

Eq. 3.1 can also be expressed in a vector equation

$$
U(x, y)=\left[\begin{array}{llll}
1 & x & y & x y
\end{array}\right]\left[\begin{array}{l}
c_{1} \\
c_{2} \\
c_{3} \\
c_{4}
\end{array}\right]
$$


If Eq. 3.11 is substituted for the terms $c_{1}$ to $c_{4}$ in Eq. 3.12, then the result can be written as

$$
U(x, y)=\left[\begin{array}{llll}
\Psi_{1} & \Psi_{2} & \Psi_{3} & \Psi_{4}
\end{array}\right]\left[\begin{array}{c}
U_{1} \\
U_{2} \\
U_{3} \\
U_{4}
\end{array}\right]
$$

or

$$
U(x, y)=\sum_{i=1}^{4} U_{i} \Psi_{i}(x, y)
$$

The equations $\Psi_{i}(x, y)$ are all bilinear equations important for bilinear interpolation.

$$
\begin{gathered}
\Psi_{1}(x, y)=\left(1-\frac{x}{a}\right)\left(1-\frac{y}{b}\right) \\
\Psi_{2}(x, y)=\left(\frac{x}{a}\right)\left(1-\frac{y}{b}\right) \\
\Psi_{3}(x, y)=\left(1-\frac{x}{a}\right)\left(\frac{y}{b}\right) \\
\Psi_{4}(x, y)=\left(\frac{x}{a}\right)\left(\frac{y}{b}\right)
\end{gathered}
$$


The general bilinear interpolation method is formulated for a two dimensional surface function $U(x, y)$. In image warping, when point $(x, y)$ is given in the unwarped image we need to find its corresponding point in the warp image plane. There is no explicit two dimensional surface function $U(x, y)$. However, the displacements of four vertices for the vectors shown in Fig. 3.1 (b) as $U_{1}, U_{2}, U_{3}$, and $U_{4}$, can be regarded as the values of a surface $U$ at the four vertices. Since $U$ is a vector having $\mathbf{x}$ and $\mathbf{y}$ components; we should write

$$
U=\left(U_{x}, U_{y}\right)
$$

$U_{x}$ is a $\mathrm{x}$-axis displacement to be added to $\mathrm{x}$ to find its corresponding location in the warp plane. $U_{y}$ is a $y$-axis displacement. Thus, the new location $\left(x^{\prime}, y^{\prime}\right)$ is given by

$$
\begin{aligned}
& x^{\prime}=x+U_{x}(x, y) \\
& y^{\prime}=y+U_{y}(x, y)
\end{aligned}
$$

The unknown coefficients $c_{1}, c_{2}, c_{3}$, and $c_{4}$ must be calculated by Eq. 3.11 using $\mathrm{x}$-axis displacements for $U_{x}$ and y-axis displacement for $U_{y}$. 


\subsubsection{New Bilinear Interpolation}

It was discussed that the general bilinear interpolation algorithm can be extended to the problem of grid warping as shown in Eq. 3.20 and Eq. 3.21. However, this approach require calculating the unknown coefficient twice for one grid. Also the calculations involved are cumbersome and not as efficient. In order to perform the same bilinear interpolation in a much simpler way, a new bilinear interpolation was formulated for the warping of a grid of rectangles. The importance of using this algorithm is to achieve minimum computation time thus maximumizing speed of the algorithm. The standard bilinear interpolation algorithm required more computational time because it only accounted for the warping of a single block and not for adjacent blocks.

The bilinear interpolation again uses a rectangular area as the control grid. Fig. 3.2 shows the initial rectangular grid containing points $U_{1}, U_{2}, U_{3}$, and $U_{4}$ and the final grid containing points $U_{1^{\prime}}, U_{2^{\prime}}, U_{3^{\prime}}$, and $U_{4^{\prime}}$.
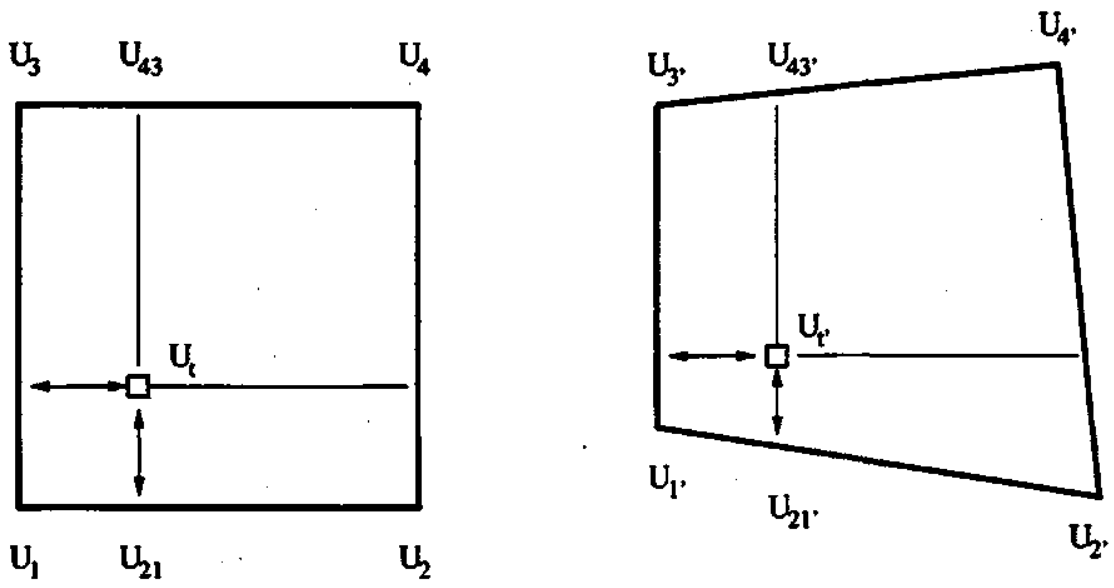

Figure 3.2 The Grid for One Warped Rectangle

The purpose of the algorithm is to take one point in the initial rectangle and find its new corresponding position in the final rectangle. To cycle through all the points in the source rectangle, two variables $\alpha$ and $\beta$ are used. These variables calculate the ratio of the lengths of one side versus a specific point. 
For the point $U_{t}, \alpha$ is calculated as the ratio of the horizontal distances.

$$
\alpha=\frac{U_{t x}-U_{1 x}}{U_{2 x}-U_{1 x}}
$$

In Fig. 3.2, $\alpha$ is represented the normalized $U_{t}$ x-coordinate position relative to $U_{1}$. The $\mathrm{x}$-coordinate distance from $U_{1}$ to $U_{2}$ is the normalizing length.

$\beta$ is the calculated ratio of the vertical distances of $U_{t}$ and the vertical displacement from $U_{1}$ to $U_{3}$.

$$
\beta=\frac{U_{t y}-U_{1 y}}{U_{3 y}-U_{1 y}}
$$

In Fig. 3.2, the choice of $\beta$ depends upon the y-coordinate location of $U_{t}$. It should be noted that the calculated values of $\alpha$ and $\beta$ change for every position of $U_{t}$.

Using this information the algorithm simply takes a point in the initial image and relocates it based on the ratios $\alpha$ and $\beta$ and the lengths of the final grid. To find the destination coordinates of $U_{t^{\prime}}$, the destination coordinates of two mid-points, $U_{21}$ (a mid-point between $U_{2}$ and $U_{1}$ ) and $U_{43}$ (a mid-point between $U_{4}$ and $U_{3}$ ) must be found first using the ratio of $\alpha$.

$$
\begin{aligned}
& U_{21 x^{\prime}}=\alpha\left(U_{2 x^{\prime}}-U_{1 x^{\prime}}\right)+U_{1 x^{\prime}} \\
& U_{21 y^{\prime}}=\alpha\left(U_{2 y^{\prime}}-U_{1 y^{\prime}}\right)+U_{1 y^{\prime}} \\
& U_{43 x^{\prime}}=\alpha\left(U_{4 x^{\prime}}-U_{3 x^{\prime}}\right)+U_{3 x^{\prime}} \\
& U_{43 y^{\prime}}=\alpha\left(U_{4 y^{\prime}}-U_{3 y^{\prime}}\right)+U_{3 y^{\prime}}
\end{aligned}
$$

These four equations calculate the points $U_{21^{\prime}}$ and $U_{43^{\prime}}$ which represent corresponding points of $U_{21}$ and $U_{43}$ in the final rectangle (see Fig. 3.2). A line connecting $U_{21^{\prime}}$ and $U_{43^{\prime}}$ represents all points that have the same $\alpha$ as $U_{t}$. 
To find $U_{t}$ in the destination grid corresponds to $U_{t}$ in the source grid, the line $U_{21^{\prime}}-U_{43^{\prime}}$ must be divided with the vertical ratio of $\beta$ according to the following equation.

$$
\begin{aligned}
& U_{t x^{\prime}}=\beta\left(U_{43 x^{\prime}}-U_{21 x^{\prime}}\right)+U_{21 x^{\prime}} \\
& U_{t y^{\prime}}=\beta\left(U_{43 y^{\prime}}-U_{21 y^{\prime}}\right)+U_{21 y^{\prime}}
\end{aligned}
$$

These calculations should give the corresponding point of $U_{t^{\prime}}$ in final rectangle position. At this point the coordinates of the point $U_{t^{\prime}}$ have now been found, the only thing left to do is copy the contents of $U_{t}$ to $U_{t^{\prime}}$. Since the grids actually contain image information (gray scale or color), the movement of the pixels can produce movement to the image. 


\subsubsection{Triangular Bilinear Interpolation using the New Algo- rithm}

The theory for triangular grids in very similar to the rectangular grid methods. The triangle consists of three vertices $U_{1}, U_{2}$, and $U_{3}$ as shown in Fig. 3.3 for the source triangle. The source triangle is used to calculate $\alpha$ and $\beta$ for the given pixel $U_{t}$.
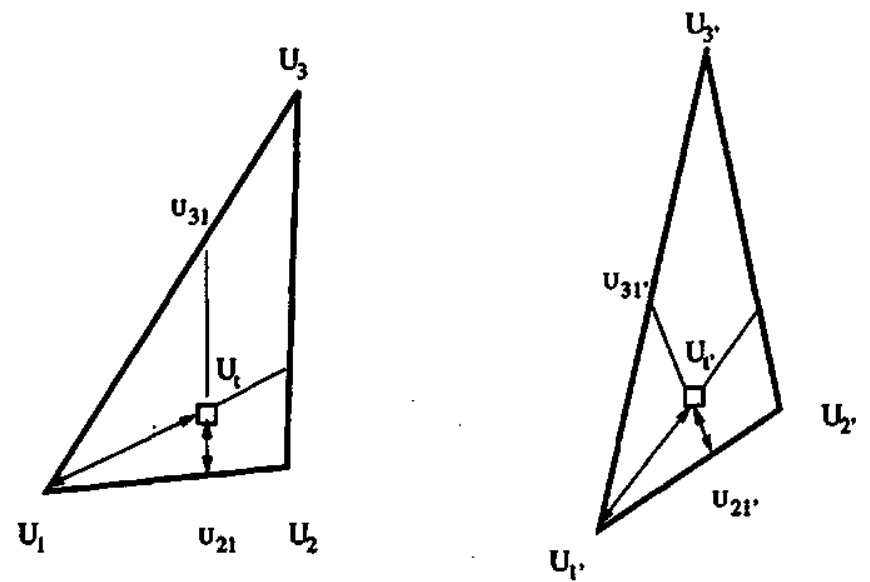

Figure 3.3 Warping of a Triangular Grid

$\alpha$ is the ratio of the x-coordinate lengths from $U_{1}$ to points $U_{t}$ and $U_{2}$.

$$
\alpha=\frac{U_{t x}-U_{1 x}}{U_{2 x}-U_{1 x}}
$$

The value of $\beta$ is the y-coordinate ratio of $U_{t}$ and $U_{3}$ to the reference $U_{1}$.

$$
\beta=\frac{U_{t y}-U_{1 y}}{U_{3 y}-U_{1 y}}
$$


These two ratios are used to find the point $U_{t^{\prime}}$ in the destination triangle. The use of these ratios must stipulate that the point of reference $U_{1}$ be positioned above or below the other two points. The reason for this $\alpha$ and $\beta$ only consider displacement in one dimension across the triangle. First $\alpha$ is used to find the line with end points $U_{31^{\prime}}$ and $U_{21^{\prime}}$. The two sets of equations which apply are Eq. 3.29 and Eq. 3.30.

$$
\begin{aligned}
& U_{21 x^{\prime}}=\alpha\left(U_{2 x^{\prime}}-U_{1 x^{\prime}}\right)+U_{1 x^{\prime}} \\
& U_{21 y^{\prime}}=\alpha\left(U_{2 y^{\prime}}-U_{1 y^{\prime}}\right)+U_{1 y^{\prime}} \\
& U_{31 x^{\prime}}=\alpha\left(U_{3 x^{\prime}}-U_{1 x^{\prime}}\right)+U_{1 x^{\prime}} \\
& U_{31 y^{\prime}}=\alpha\left(U_{3 y^{\prime}}-U_{1 y^{\prime}}\right)+U_{1 y^{\prime}}
\end{aligned}
$$

To find the coordinates of $U_{t^{\prime}}$ on this line Eq. 3.31 is used.

$$
\begin{aligned}
& U_{t x^{\prime}}=\beta\left(U_{31 x^{\prime}}-U_{21 x^{\prime}}\right)+U_{21 x^{\prime}} \\
& U_{t y^{\prime}}=\beta\left(U_{31 y^{\prime}}-U_{21 y^{\prime}}\right)+U_{21 y^{\prime}}
\end{aligned}
$$

The number of calculations in the triangle method are the same as the rectangular method. However, the use of triangles allows for more flexibility in grid movement. The rectangular method requires the reference grid to be rectangular, but not any four sided polygon; the triangle grid is not restricted by its shape. 


\subsection{Cubic Spline Interpolation}

The Cubic Spline method was designed for high quality warping of images. The cubic spline is a curve fitting method which interpolates unknowns between known data using a nonlinear (3rd order polynominal) function. Since the method warps line by line, the warping appears to be smoother whereas the bilinear polygon method tends to form ridges between adjacent polygons. This method can produce warped images compatible with those produced by the bilinear method so that it can be easily substituted as an alternative warping routine. But, the warping needs to be implemented cautiously since it fails if grid nodes occupy exactly the same position. The source code from Wolberg [5] was modified and used for this project.

This algorithm requires input image data, a overlaid $\mathrm{S}$ (source) grid, and $\mathrm{D}$ (destination) grid. In using the cubic spline algorithm the perimeter of the grid must have restricted movement. The top and bottom lines of the grid must be considered fixed for vertical motion, and the left and right lines cannot move horizontally. All four corner points must not move at all. If local warping is needed, such as for an eye, the section can be separately warped by a local grid. Warping in sections can increase the speed of the cubic spline method, but increases the complexity of the grid.

This method is a two pass method where horizontal warping is preformed on the image first, then the vertical warping is done. Thus intermediate storage is required to keep the result of the horizontal warping. The $S$ and $D$ grids are shown in the fields of motion in Fig. 3.4. Using the number of horizontal grid points $W_{g}$ and that of vertical grids $H_{g}$, the size of the grids can be represented by $H_{g} \times W_{g}$ whereas the image size is $H_{\text {in }} \times W_{\text {in }}$.

In the first pass the horizontal movement of source grid $S$ is accounted for. As shown in Fig. 3.5, a vertical smooth curve connecting the source grids (dotted line) must be horizontally moved to the smooth curve connecting the intermediate points (solid line). A cubic spline (solid line) is generated by vertically interpolating between the intermediate points. This process fill the gaps between $H_{g}$ grid points, producing 


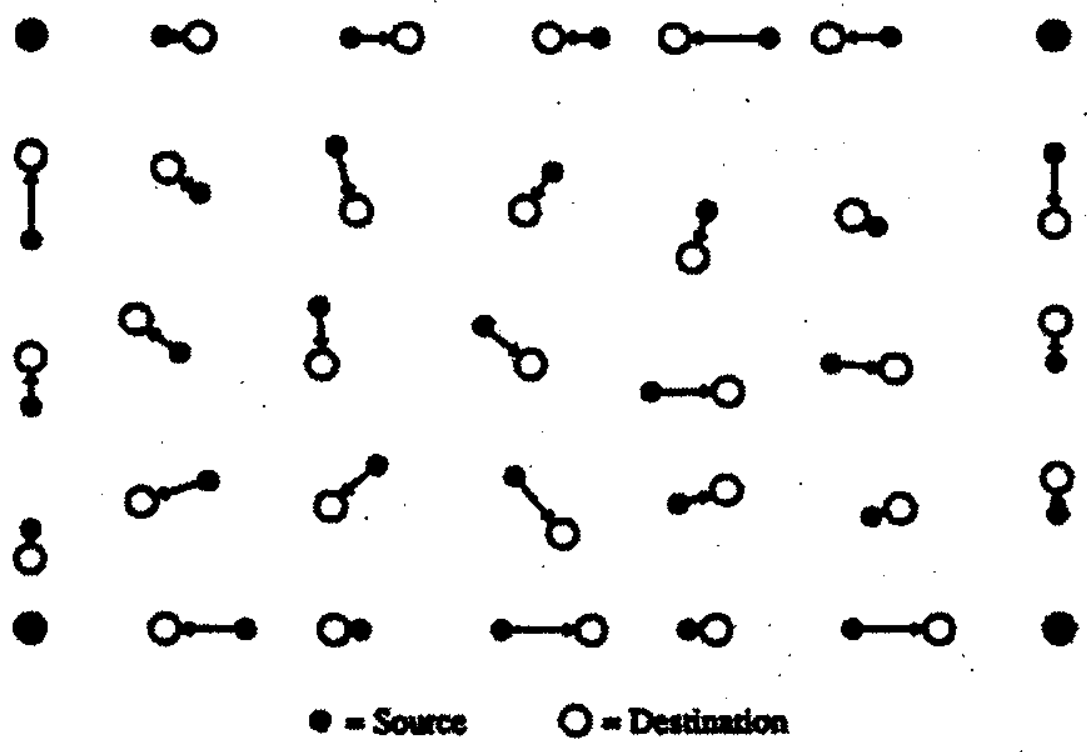

Figure 3.4 The Source and Destination Nodes

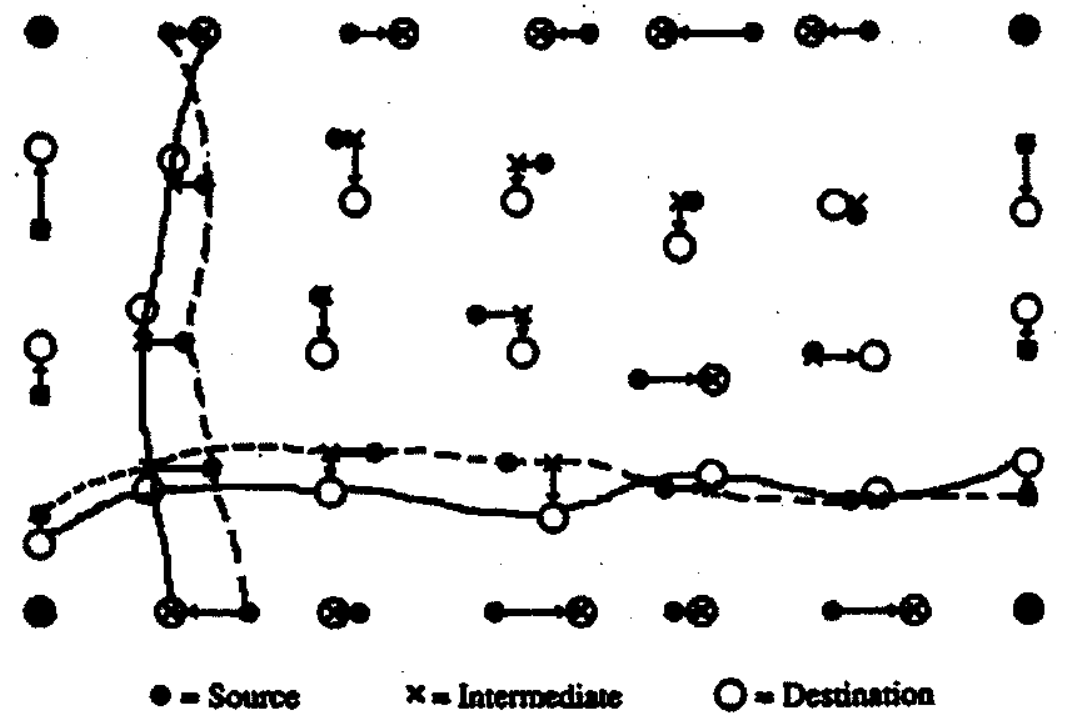

Figure 3.5 Horizontal and Vertical Movement Separation.

$H_{\text {in }}$ intermediate data points to be used in the second pass. All the resulting $H_{\text {in }}$ data, which indicate the horizontal displacement of intermediate grids for each raster scanned line of the input image, are stored in the intermediate table of $H_{i n} \times W_{g}$.

In the second pass, the vertical movement is then accounted for. A smooth curve joining the intermediate points (dotted line) now needs to be moved to the curve joining the $W_{g}$ destination grid points. A cubic spline is generated by horizontally 
interpolating the gaps between the destination points producing $W_{\text {in }}$ data points. Since $W_{g}$ grid points have been calculated for each raster scan line during the first pass, the new pixel locations of every raster scan line of the picture are thus found. At the end of the second pass, the new locations of all pixels are stored in a $H_{\text {in }} \times W_{\text {in }}$ table.

The cubic spline method is a curve fitting algorithm which produces the best spline function in the sense of the least square error. The specific type of spline used here is the Catmull-Rom spline that fit a cubic function,

$$
Y(x)=A_{3} x^{3}+A_{2} x^{2}+A_{1} x+A_{0}
$$

between two points bases on the values and the first derivatives. The source code adopted from Wolberg [5] determines the coefficients $A_{3}, A_{2}, A_{1}$, and $A_{0}$ of Eq. 3.32 by a routine called ispline_gen. The ispline_gen routine generates a fine grid of a size $H_{\text {in }} \times W_{g}$ from the grid of a size $H_{g} \times W_{g}$ in the first pass. The same ispline $g e n$ produces another table of the size $H_{i n} \times W_{i n}$ to interpolate vertical movement in the second pass.

The routine ispline_gen is designed to accept course $\mathrm{x}$ and $\mathrm{y}$ grid coordinates, and either of the fine $\mathrm{x}$ or $\mathrm{y}$ grid coordinates. The algorithm first generates all derivatives of the course grid, which are used to find the cubic spline coefficients. The derivation of the cubic spline algorithm and the original code of ispline gen are described in detail in appendix 2, pages 283 to 296, of the book by Wolberg[5].

Pixels can not be moved directly from the source locations calculated. A pixel resampling algorithm must first correct for image anomalies such as aliasing, and pixel gaps. The algorithm used by the cubic spline is resample_gen, which is based on the the Fant's resampling algorithm. 


\subsubsection{Fant's Resampling Algorithm}

The Fant's Resampling algorithm is designed for pixel reconstruction, and antialiasing problems in images. These problems arise from the compression and explansion of pixels due to the cubic spline movement. Rather than having the pixels overlap or leave gaps this method combines the pixels. Each input pixel occupies a interval of space. In a digital image all pixels have a width of 1 . After processing an image by the cubic spline, these intervals maybe larger or smaller than this. The Fant method places pixels back into the ones of an equal interval.

The process can be best illustrated by the following example. In Table 3.1 the uneven pixel coordinate values $I$ and cooresponding "Input" pixel intensities are given. $\Delta I$ is the difference between adjacent $I$ 's. The "Output" pixels are calculated for new pixel coordinate values listed as $O$ which are integer values as noticed.

Table 3.1 Fant Algorithm Example

\begin{tabular}{|l|c|c|c|c|c|c|c|c|c|l|}
\hline $\mathrm{O}$ & 0.0 & & 1.0 & & 2.0 & & & & 3.0 & \\
$\mathrm{I}$ & & 0.7 & & 1.5 & & 2.1 & & 2.6 & & 3.5 \\
$\Delta \mathrm{I}$ & 0.7 & & 0.8 & & 0.6 & & 0.5 & & 0.9 & \\
$\begin{array}{l}\text { Input Pixels } \\
\text { Output Pixels }\end{array}$ & $P_{O 0}$ & 135 & & 150 & & 156 & & 110 & & \\
\hline
\end{tabular}


To calculate the value at $I=1$, the linear interpolation between 0.7 and 1.5 gives the first order approximation

$$
135(1-\alpha)+150 \alpha=140.625
$$

with distance ratio $\alpha$

$$
\alpha=\frac{1.0-0.7}{1.5-0.7}
$$

Since this value is valid only between 0.7 and 1.5 and disregarding the interval 1.5 and the next integer value 2.0 , the weighted average of 140.625 and 145 by the distance ratio of (1.5-1.0):(2-1.5) represents the better estimate at 1.0. This is the output pixel value $P_{O 1}$. Other output pixel values are similarly calculated as follows:

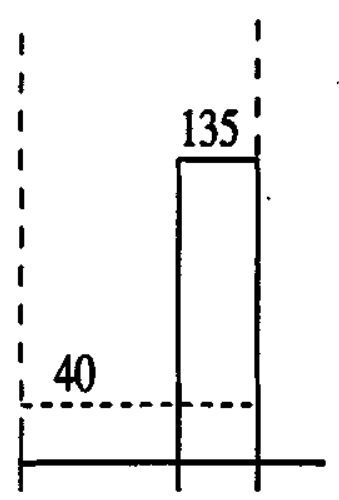

$\begin{array}{llll}0 & 0.7 & 1.0 & 0\end{array}$

(a)

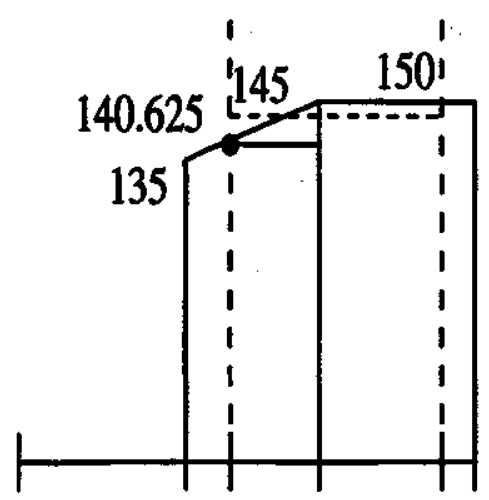

$0.71 .0 \quad 1.5 \quad 2.02 .1$

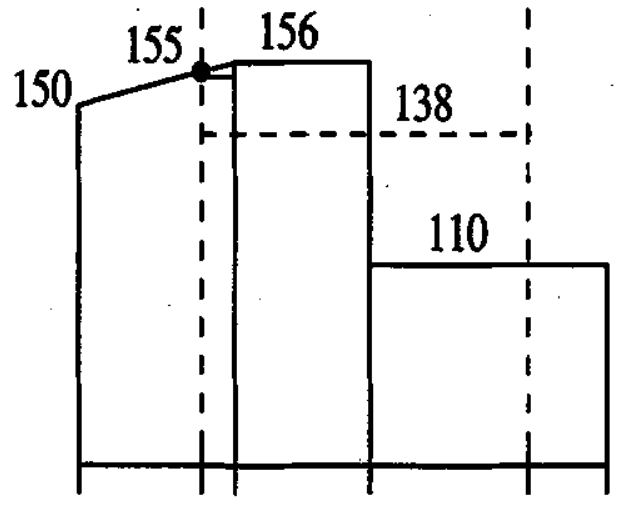

$1.5 \quad 2.02 .1 \quad 2.6$

(c)

Figure 3.6 Fant Calculation Example

$$
\begin{aligned}
& P_{O 0}=135(1.0-0.7)=40.5 \\
& P_{O 1}=\left[135\left(1.0-\frac{0.3}{0.8}\right)+150 \frac{0.3}{0.8}\right](1.5-1.0)+150(2.1-.1-1.5)=145 \\
& P_{O 2}=\left[150\left(1.0-\frac{0.5}{0.6}\right)+156 \frac{0.5}{0.6}\right](2.1-2.0)+156(2.6-2.1)+110(3.5-.5-2.6)=138 \\
& P_{O 3}=\left[156\left(1.0-\frac{0.4}{0.9}\right)+110 \frac{0.4}{0.9}\right](0.5)=68
\end{aligned}
$$




\subsection{The Application of the Warping Method}

The choice of the warping method depends upon the requirement of the application, each method described in this chapter satifies the objective of warping the image using a mesh based algorithm. Comparing the methods show that the advantage of the bilinear method is speed, and the advantage of the cubic spline is warping quality. In the interests of the thesis bilinear interpolation is the best choice for a real-time system. 


\section{Mesh Motion Control}

\subsection{Introduction}

This chapter discusses the use of the mesh to dictate the parameters related to motion described in the facial detection section of chapter 5 . In conjunction with preliminary work done in chapter 2 , which was about the use of an intricate facial mesh, this chapter discusses the various mesh techniques, that manipulate the mesh.

A rectangular grid is considered here for the mesh. The three methods described in the following sections;

1. Attractor/Repellor Motion

2. X-Y Image Motion

3. Nonlinear Scaling

are of primary importance to realize eye and mouth motion as well as head motion in warping facial images.

\subsection{The Rectangular Mesh}

The migration from the complex grid in chapter 2 to the simple rectangular grid is due to the difficulty in manipulating the complex grid. Therefore, a grid that would foster the abilities of a real-time system is more desirable. The grid should allow easier image warping and object placement of the eyes, mouth, and head. For warping, the grid should permit accurate reproduction of facial movement at the decoder. In object placement, the grids must be able to cover the eyes, mouth, and head alone at the discretion of the real image. 
A rectangular grid of $\mathrm{x}$ rows and $\mathrm{y}$ columns is thus introduced. The spacing for the rows and columns is adjusted with respect to the eyes, mouth, and head. The number of grid lines for each facial part is also set to a specific size, $20 \times 16$ for the head, $5 \times 5$ for the eyes, and $5 \times 7$ for the mouth. The location of these specific grids depends on the information extracted from the reference image. The base grid is shown in Fig. 4.1. This grid is initially an equally spaced rectangular grid. Upon entry of the eyes, mouth, and head parameters the horizontal and vertical lines are then positioned according to the information regarding the facial feature.

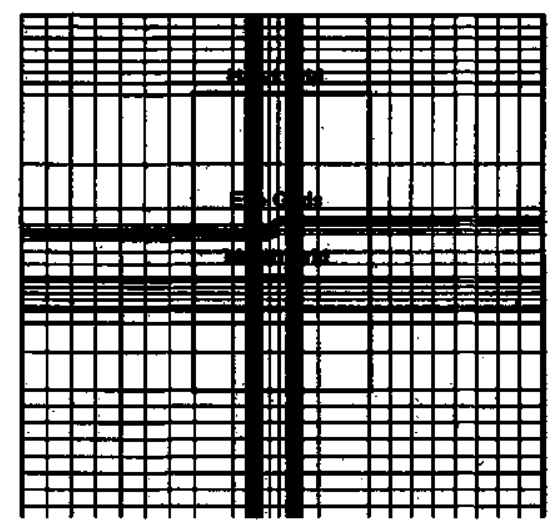

Figure 4.1 The Base Rectangular Grid

\subsubsection{Improved Grid Structure}

Using the base grid in Fig. 4.1 is adequate to achieve the objectives of mesh movements. Yet, a few modifications can improve the performance of the decoder. One problem is in grid overlapping. Grid overlapping usually results in the appearance of a fold in the image. In the grid shown in Fig. 4.1, the vertical grid lines of mouth are extended beyond the mouth area and overlaps those of the eyes unless the mouth is positioned exactly between the two eyes.

Another problem is CPU time wasted on the calculation of rectangular grids that do not move. In Fig. 4.1, closely spaced grid lines for the eyes and mouth unnecessarily subdivide the head area into smaller blocks. This problem can be solved by setting up a local fine grid for the eyes and mouth without extending the grid lines across the face area. Fig. 4.2 shows how the grid in Fig. 4.1 was modified. The new grid 
saves time and reduces the image folding when the bilinear image warping is used.

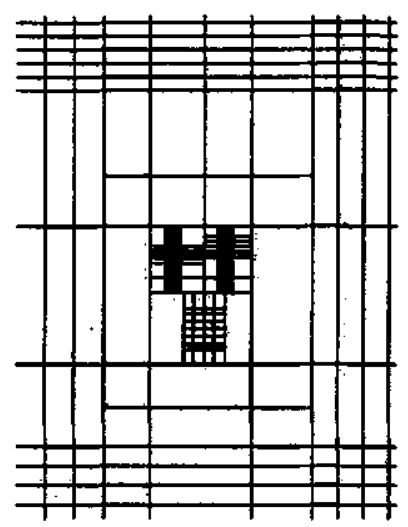

Figure 4.2 The Modified Rectangular Grid

\subsection{Introduction to Mesh Movements}

To reduce the amount of data to be sent to the decoder, the encoder must convert facial information into simple parameters. The decoder then will translate these parameters back into mesh movements. The process involves the encoder, which provides parameters for the decoder. This section looks at the movement parameters from the perspective of the decoder in order to make the encoder specifications well defined and concrete.

\subsection{Attractor/Repellor Mesh Warping}

The Attractor/Repellor algorithm simulates eyes and mouth motion. The motion that occurs in a local grid can be manipulated by this warping method. To produce a realistic simulation mesh lines should not overlap each other. Otherwise, an overlap area painted first will be overwritten and hidden by another overlapping grid. 


\subsubsection{Theory of Operation}

The method is based on the simple physics between positively and negatively charged bodies. These bodies in the Attractor/Repellor method are called grid nodes and mass nodes. The grid nodes are the points of intersection of the grid. Mass nodes are independent bodies placed in and around the mesh. The interaction between the two bodies, being attraction or repulsion, is the basis of the mesh movement. In the first case, attraction, the mass nodes are of a different charge than the grid nodes. The reactions between the bodies would be the grid nodes pulling in toward the mass nodes while the links between the nodes are constrained keeping their distance from other grid nodes. Fig. 4.3 shows how this interaction would occur for a $5 \times 5$ grid with a mass point at position $(3.5,3.0)$.

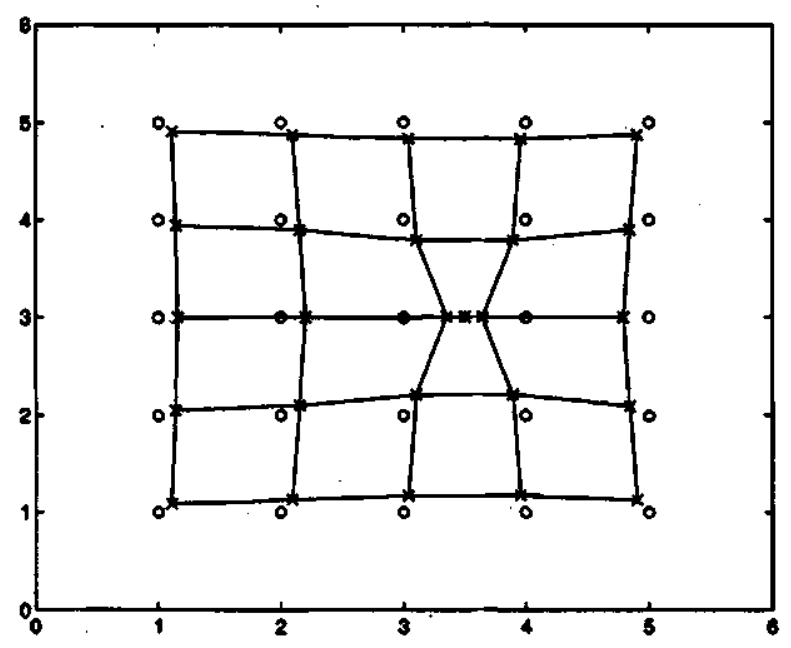

Figure 4.3 Example of Grid Nodes Attracting to the Mass node $(3.5,3.0)$ 
In the second case, repulsion, the grid nodes and the mass nodes have the same charge. The reaction of the bodies is the pulling away of grid nodes from the mass nodes. The reaction of the $5 \times 5$ grid with the same single mass point is shown in Fig. 4.4. The mass node forces are always stronger for grid nodes near the mass point and get weaker for those nodes located further away.

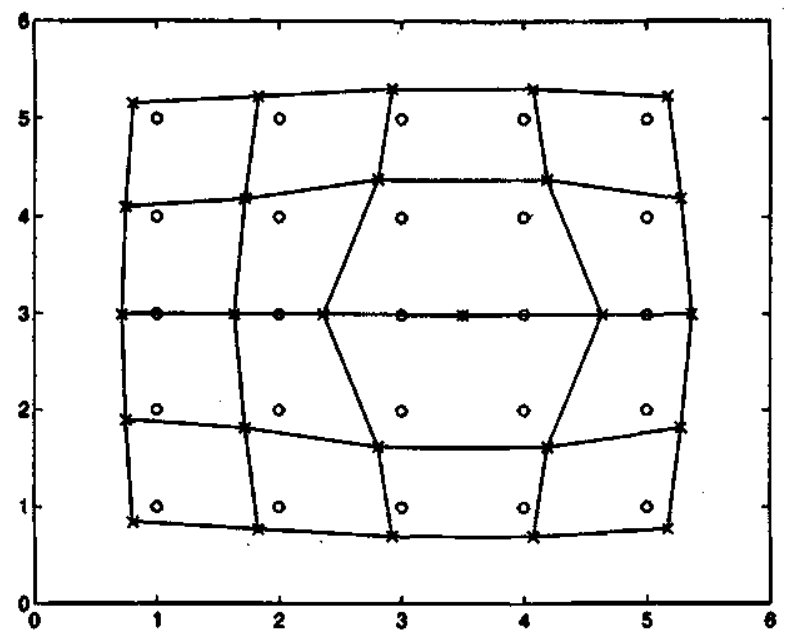

Figure 4.4 Example of Grid Notes Repelling from the Mass Node $(3.5,3.0)$

\subsubsection{Displacement of Grid Nodes}

The mass nodes are the essential points set to achieve the desired warping effect. The effects of the mass nodes depends on placement, charge, and number of nodes. The displacement of the grid nodes is expressed by

$$
d \mathbf{r}=\frac{\mathbf{r}}{|\mathbf{r}|^{n}} \quad \mathbf{r} \geq 1
$$

Vector $\mathbf{r}$ represents the distance between a mass node and a grid node. The variable $d \mathbf{r}$ is the vectorial increment added to the original distance $\mathbf{r}$. $n$ decides the degree of movement with this inverse distance relationship. The formula indicates that the incremental vector is inversely proportional to the $n$th power of the vector length. Direction of the vector will not change. 
In the software, vectors are written as complex numbers. For each grid node the Attractor/Repellor calculations are organized as illustrated in the following pseudo code.

1 For $\mathrm{i}=1$ to number of mass nodes.

$2 r=$ distance between selected mass node and grid node.

$3 \mathrm{D}=$ absolute value of $\mathbf{r}$

$4 d \mathbf{r}=d \mathbf{r}+\frac{\mathbf{r}}{D^{n}}$

5 end loop

$6 \quad d \mathbf{r}=\beta\left(d \mathbf{r}_{x}\right)+j \alpha\left(d \mathbf{r}_{y}\right)$

7 Add $d \mathbf{r}$ to grid node

For calculations of a specific grid node movement $(d \mathbf{r})$, each mass node is applied separately to find its $d \mathbf{r}$ term. The net $d \mathbf{r}$ displacement for the grid node is then vectorially added to $\mathbf{r}$. The Attractor/Repellor pseudo code shows four parameters necessary in controlling of grid motion, the mass node, $n, \alpha$, and $\beta$. The mass nodes are important for controlling the direction and pattern of motion. $n$ controls the intensity of motion, $\alpha$ and $\beta$ provide directional control. Each of these terms will now be discussed.

Mass Nodes The use of mass nodes depends on the objective of the mesh movement. One mass node can cause uniform movement of grid nodes in all directions from the mass. Operations that are more specific to the shape of an object may require movement in one or more chosen directions. Chapter 6 will show basic motion control, and the warped images resulting from a different number of mass nodes. 
n The choice of $n$ determines the degree and distribution of grid node movement. With low values of $n(n<1.5)$ the inner grid nodes around the mass node move with a moderate distance, and become close to other grid nodes that are moving in the same direction. Hence, the grid nodes maintain a reasonable distance from each other. For this thesis the proper $n$ will be investigated.

$\alpha$ and $\beta$ Facial movements such as in the eyes and mouth do not always move equally in all directions. The use of $\alpha$ and $\beta$ is to control the relative motion in the horizontal and vertical directions. $\beta$ is the controlling factor for $\mathrm{x}$ directional displacement, and $\alpha$ is for $\mathrm{y}$ directional displacement. In the following picture $\beta$ was set to 2 and $\alpha$ was set to 1 .

$$
d \mathbf{r}=\beta\left(d \mathbf{r}_{x}\right)+j \alpha\left(d \mathbf{r}_{y}\right)
$$

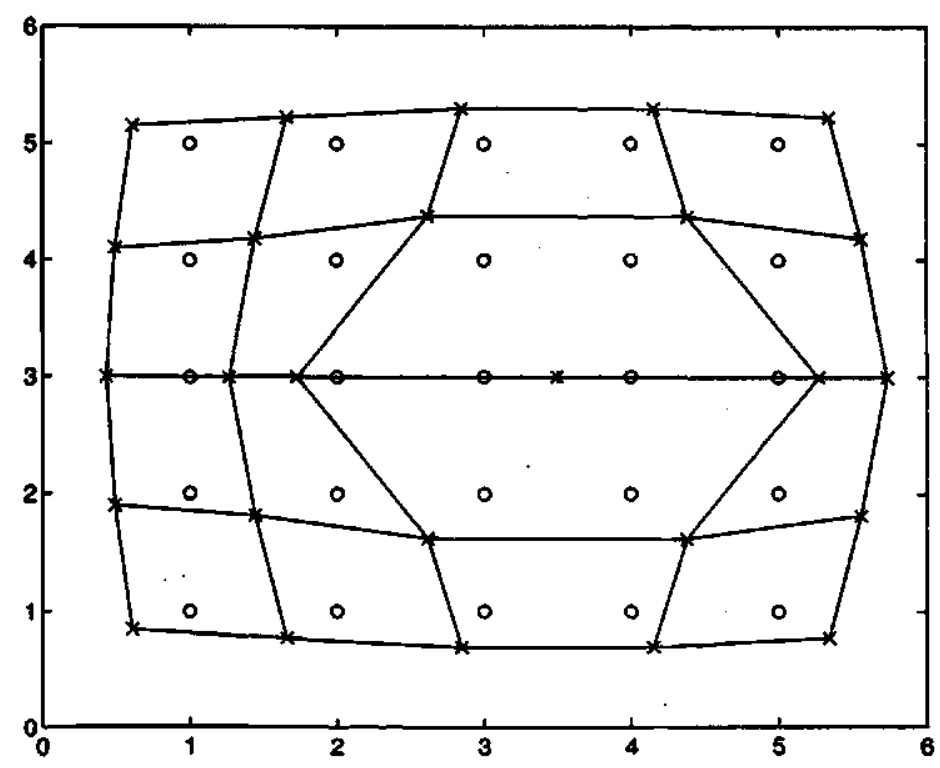

Figure 4.5 A Repelling Grid with $\beta=2.0$ and $\alpha=1.0$ 


\subsection{X-Y Mesh Motion}

The motion that affects the 2-D plane is discussed in this section. The methods used are similar to those discussed in section 2.4 "Controlling of the Action Units". The use of this form of mesh motion is only for head motion.

\subsubsection{X-Y Rotation}

The objective of rotation is to produce a simulated swaying of the head. The theory of head rotation is based on vector rotation where a pivot point $\left(x_{c}, y_{c}\right)$ and the angle of rotation $\theta$ are required for input. All nodes that make up a head must move in the same direction as given by the parameters. The formula for this rotation is

$$
\left[\begin{array}{c}
y^{\prime}-y_{c} \\
x^{\prime}-x_{c}
\end{array}\right]=\left[\begin{array}{rr}
\cos (\theta) & \sin (\theta) \\
-\sin (\theta) & \cos (\theta)
\end{array}\right]\left[\begin{array}{l}
y-y_{c} \\
x-x_{c}
\end{array}\right]
$$
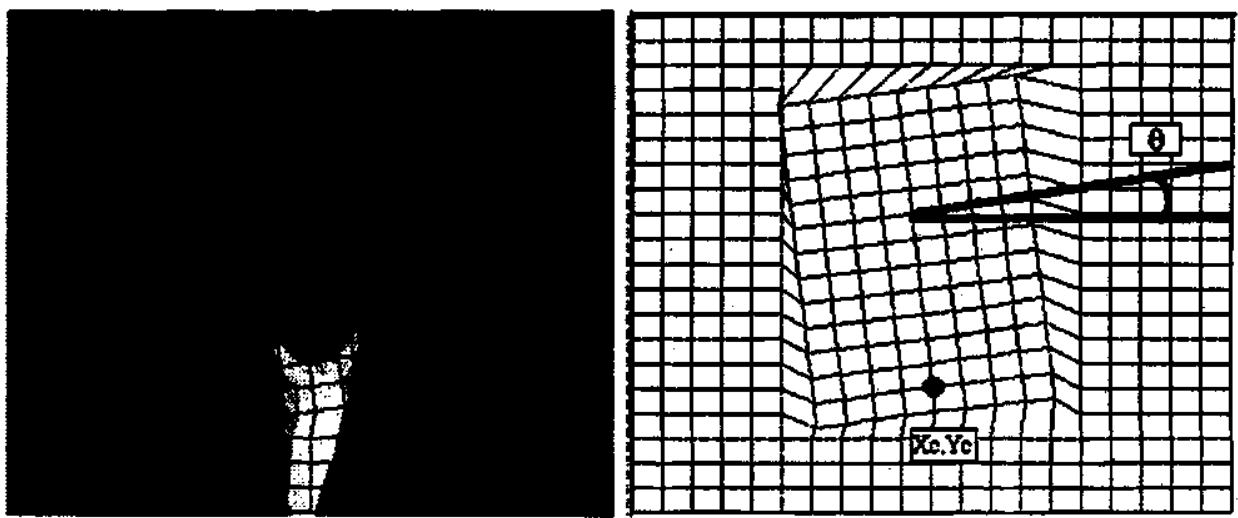

Figure 4.6 Action of X-Y Head Rotation 


\subsubsection{X-Y Displacement}

$\mathrm{X}-\mathrm{Y}$ displacement becomes necessary to realize a simple translation movement that occurs in the head and body. The movement is performed by the addition or subtraction of the offset value $\left(d_{x}, d_{y}\right)$ from the head mesh.

$$
\begin{aligned}
& x^{\prime}=x+d_{x} \\
& y^{\prime}=y+d_{y}
\end{aligned}
$$

\subsubsection{X-Y Zooming (Linear Scaling)}

Zooming is the resizing of an object, which occurs when a face is moving toward or away from a camera. Eq. 4.6 must be applied to all head block nodes for zooming. $\left(y_{z}, y_{z}\right)$ is a reference point that all zooming is related to, it is usually set at the center of the face. To increase or decrease the size a scaling factor must be multiplied. $A_{x}$ is for horizontal scaling, and $A_{y}$ for vertical scaling.

$$
\left[\begin{array}{c}
x^{\prime}-x_{z} \\
y^{\prime}-y_{z}
\end{array}\right]=\left[\begin{array}{cc}
A_{x} & 0 \\
0 & A_{y}
\end{array}\right]\left[\begin{array}{l}
x-x_{z} \\
y-y_{z}
\end{array}\right]
$$

The x-scaling factor $A_{x}$ and y-scaling $A_{y}$ can be different but these values are set equal for normal zooming effect, i.e. $A_{x}=A_{y}$. 


\subsection{Nonlinear Scaling}

The use of nonlinear scaling is to produce the simulated action of 3-D head rotation with respect to one of the two image coordinates, say $x$-axis or $y$-axis. The rotation of the head about either $x$-axis or $y$-axis may be described as the 2-D expansion and contraction of a face. This is accomplished by changing the distances between grid lines nonlinearly from one side of the grid to the other side. The 3-D head is positioned as shown in Fig 4.7 with the z-axis representing depth. The reference position of the head is the face is looking straight ahead.

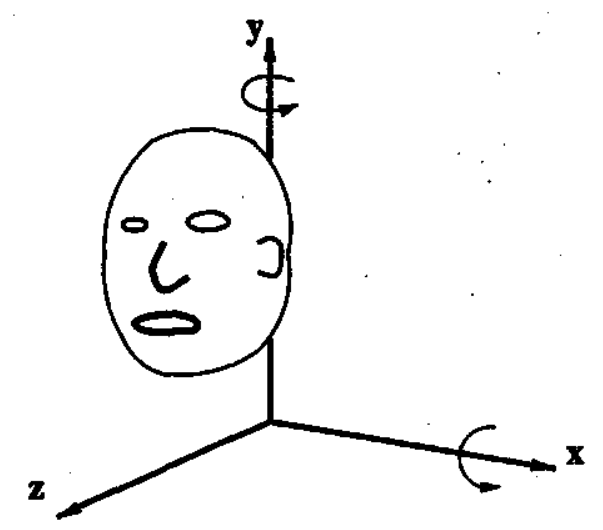

Figure 4.7 Persons head in 3D Dimensions

In $\mathrm{X}-\mathrm{Z}$ plane rotation about the $\mathrm{y}$-axis the facial features move in a horizontal direction. This is the movement right or left. As the head rotates from the reference position the relative distance between facial objects decreases as perceived by the camera. For example, the distance between the eyes in the 2-D image would decrease as the head rotates to the right or left. For $\mathrm{Y}-\mathrm{Z}$ rotation about the $\mathrm{x}$-axis, the same will occur but with respect to the vertical distance between the facial features.

To illustrate this action, Fig. 4.8 shows the movement of grid lines within a head block. In the first grid, where all grid lines are equally spaced, there are three highlighted nodes. The points $\left(x_{1}, y_{1}\right)$, and $\left(x_{0}, y_{0}\right)$ are the bounding nodes around the head, being the upper right, and lower left positions. These points represent a fixed boundary. Rotation of the head is represented by the movement of $\left(x_{i}, y_{i}\right)$, to $\left(x_{i}^{\prime}, y_{i}^{\prime}\right)$. In this example both $\mathrm{x}$ and $\mathrm{y}$ movement pushes all grid lines down and towards 
the right. This method is called nonlinear because grid lines must be redistributed nonlinearly with the compaction and expansion of the mesh.

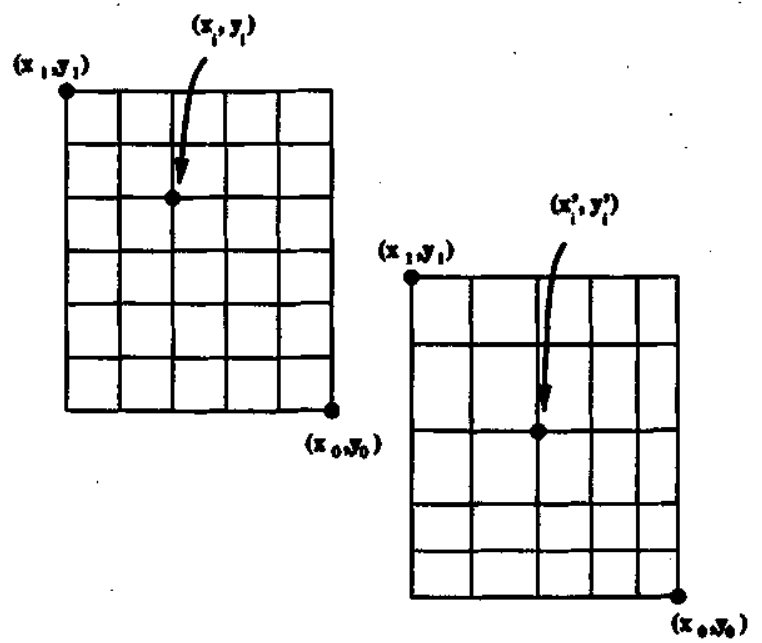

Figure 4.8 NonLinear Scaling Head Block Nodes

The simplest form of nonlinear scaling is a quadratic equation given by Eq. 4.7. The values $y_{1}, y_{i}$, and $y_{0}$ represent three points on the grid. $y_{1}$ and $y_{0}$ are the boundary values at the left edge and right edge for X-Z rotation. $y_{i}$ is a moving point of motion, when the head is rotated $y_{i}$ moves to $y_{i}^{\prime}$. The coefficients of the quadratic equation $a, b$, and $c$ can be determined as follows. 
Coefficient $a$ represents the direction and amount of movement.

$$
y_{i}^{\prime}=a y_{i}^{2}+b y_{i}+c
$$

Since $y_{i}^{\prime}=y_{0}$ when $y_{i}=y_{0}$, and $y_{i}^{\prime}=y_{1}$ when $y_{i}=y_{1}$.

$$
\begin{aligned}
& y_{0}^{\prime}=a y_{0}^{2}+b y_{0}+c \\
& y_{1}^{\prime}=a y_{1}^{2}+b y_{1}+c
\end{aligned}
$$

$a$ is an arbitrary constant, i.e. $a=a_{y}$. Subtracting Eq. 4.9 from Eq. 4.8 yields

$$
b=\frac{y_{1}-a y_{1}^{2}-y_{0}+a y_{0}^{2}}{y_{1}-y_{0}} .
$$

Thus,

$$
c=y_{0}-a y_{0}^{2}-b y_{0}=y_{1}-a y_{1}^{2}-b y_{1}
$$

If $a, b, c$ are substituted into Eq. 4.7, it now becomes

$$
y_{i}^{\prime}=a_{y}\left(y_{i}^{n}-y_{0}^{n}\right)+\left(y_{1}-y_{0}-a_{y}\left(y_{1}^{n}-y_{0}^{n}\right)\right) \frac{y_{i}-y_{0}}{y_{1}-y_{0}}+y_{0}
$$

Another set of equations for the $\mathrm{x}$-axis derives

$$
x_{i}^{\prime}=a_{x}\left(x_{i}^{n}-x_{0}^{n}\right)+\left(x_{1}-x_{0}-a_{x}\left(x_{1}^{n}-x_{0}^{n}\right)\right) \frac{x_{i}-x_{0}}{x_{1}-x_{0}}+x_{0}
$$

Where $n$ equals 2 in the second order case. Testing will be done to determine the affect of changing $n$. 
The control of how much rotation is desired depends on the value of $a_{x}$ and $a_{y}$ for the X-Z and Y-Z rotations respectively. When $a_{x}$ is positive the movement is towards the right, when it is negative the movement is towards the left. As for $a_{y}$, a positive value is for down motion and a negative value is for an upward motion. To illustrate the motions of the head, Fig. 4.9 shows a basic head rotation to the right when $a_{x}>0$. In Fig. 4.10 the downward motion of the head is shown when $a_{y}>0$. The simulation of the head rotation is limited to movement within the bounds of the head. Side to side rotation greater than some small angle (such as $15^{\circ}$ ) is not practically possible using these mesh movements because of the limitations of the perspective of the image used.
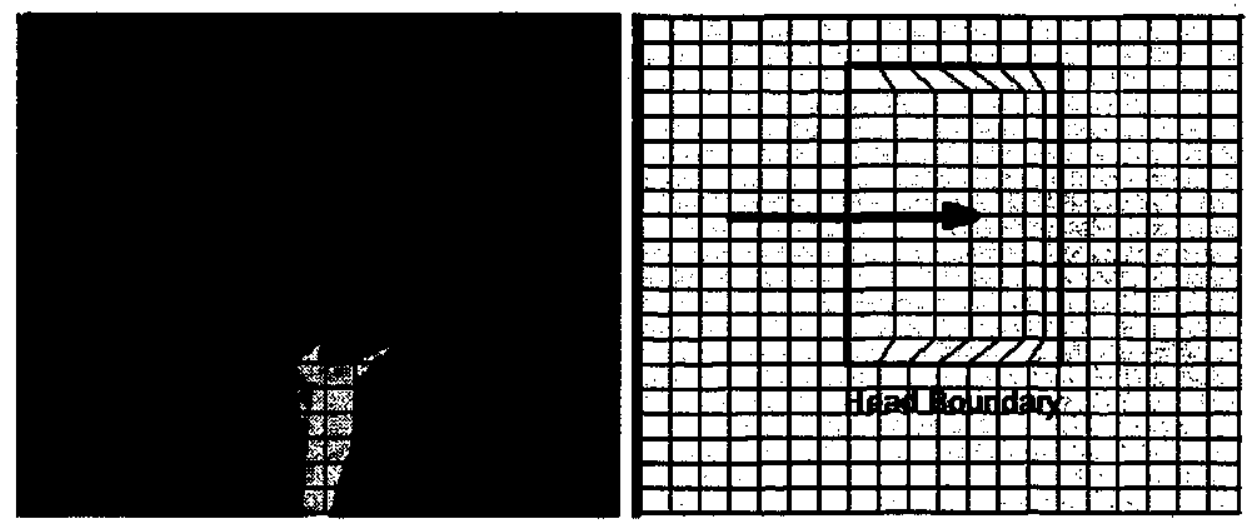

Figure 4.9 Action of X-Z Head Rotation
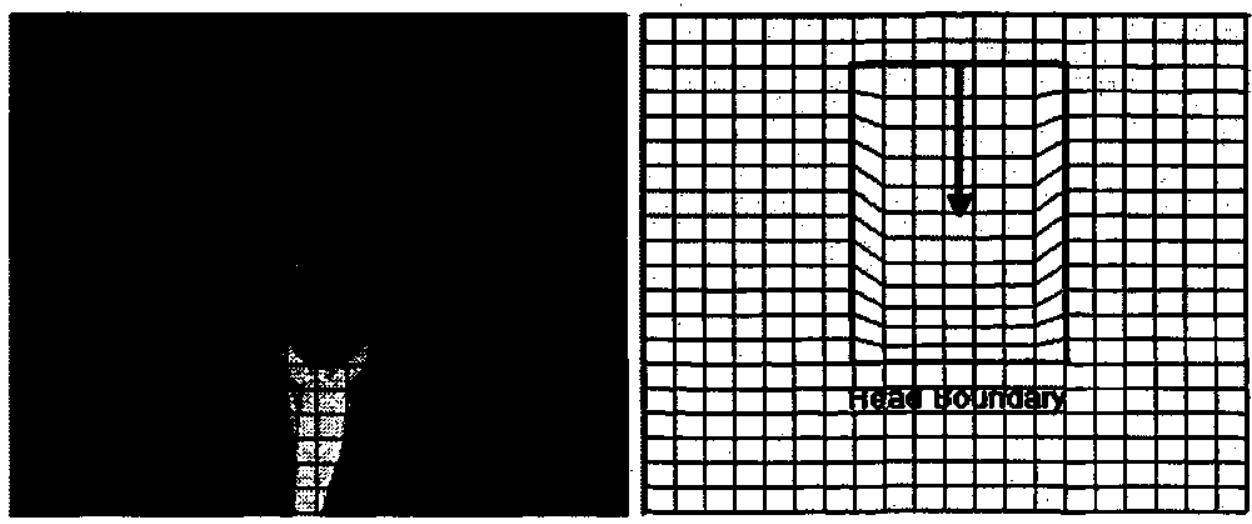

Figure 4.10 Action of Y-Z Head Rotation

For the operation of nonlinear scaling, grid nodes are mapped by a quadratic equation. The values of $a_{x}, a_{y}$, and $n$ control the movement. These parameters will be tested and given in chapter 6 . 


\section{Facial Feature Detection}

\subsection{Digital Image Filtering}

Image filtering is the extraction or removal of information in an image. There are many different types of filters available, but the filter operation is very much the same. In this section, the method extracting edge information from a picture is the focus of image filtering. The choice of filter is based on its ability to extract edge features in the face. The three types of filtering covered are Pixel Differentiation, Template filtering, and Laplacian filtering.

\subsubsection{How a Image Filter Works}

Image filtering is realized by discrete convolution. A given image is convoluted with a filter matrix, which is much smaller than the size of the image as shown in Fig. 5.1. The operation simply scans the large image with the mask of a filter matrix, calculating the sum of pixel products between the image and the filter.

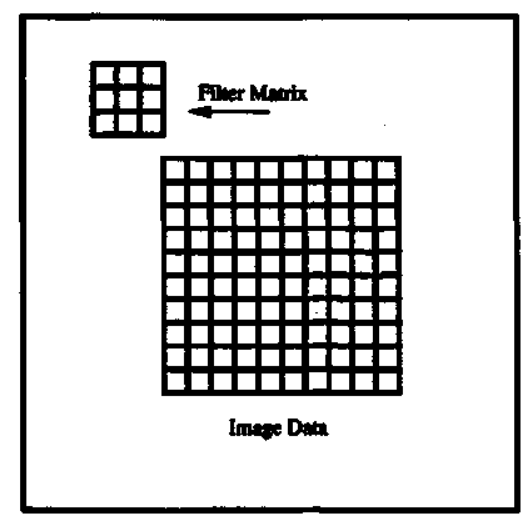

Figure 5.1 The Grids used in Convolution

The process requires a square filter of odd sizes only as the filter requires a center component. This center component is the position acted upon, and all adjacent pixels influence the filter's result. Standard filter sizes are $3 \times 3,5 \times 5$, and $9 \times 9$. The size of 
$3 \times 3$ is the most common since it requires the fewest calculations. The images used in filtering are eight bits per pixel for gray scale pictures. Convolution of whole number may produce values outside the pixel range of 0 to 255 . Values that fall outside the range need to be normalized using a scale factor, which is the inverse of the sum of the filter's components.

A 1-D convolution of the image data is shown in the following example.

The scale factor is set to $1 / 4$.

$\begin{array}{lrllllll}\text { Input Array } & \text { A } & 50 & 75 & 75 & 25 & 10 & 5 \\ \text { Mask Array } & \text { B } & 1 & 2 & 1 & & & \\ & \text { A } \otimes B & 12 & 44 & 69 & 62 & 34 & 12\end{array}$

For a 2-D grid the number of calculations are increased by a factor of two as shown in Fig. 5.2. In this example, the $3 \times 3$ filter is overlapped with the top right corner such that the 4 in the source image matrix aligns with the center of the filter. The overlapping sections are multiplied and then added, this value is then divided by a scaling factor $1 / 4$. The destination image matrix highlights the resulting answer $1 / 4$.

\begin{tabular}{|c|c|c|}
\hline-2 & -1 & 0 \\
\hline-1 & 0 & 1 \\
\hline 0 & 1 & 2 \\
\hline
\end{tabular}

Filter

\begin{tabular}{|l|l|l|l|l|l}
\hline 2 & 3 & 5 & 6 & & \\
\hline 8 & 4 & 5 & 2 & & \\
\hline 1 & 2 & 8 & 1 & & \\
\hline 3 & 0 & 2 & 5 & & \\
\hline & & & & &
\end{tabular}

Source Matrix

\section{$1 / 4((-2 \times 4)+(-1 \times 5)+(0 \times 2)+(-1 \times 2)+(0 \times 8)+$}

$(1 \times 4)+(0 \times 0)+(1 \times 2)+(2 \times 5))=1 / 4$

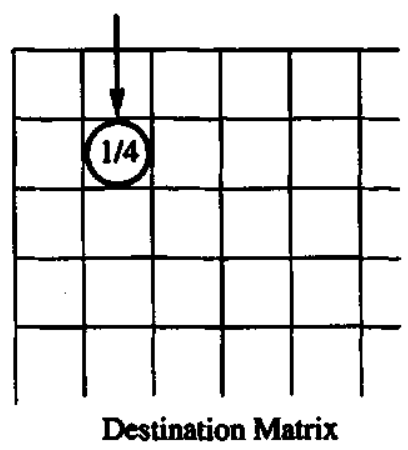

Destination Matrix

Figure 5.2 Convolution of an $3 \times 3$ Filter and the Image Matrix 


\subsubsection{Edge Detection Filtering}

For the detection of the facial features, edges must be enhanced to detect the important features in the image. The types of filtering used are first derivative filters and second derivative filters.

\subsubsection{First Derivative Filters}

The first order filters are technically gradient generators, which is designed to detect a gradient in a specific direction. There are two types of generators, the orthogonal gradient generators, and the edge template gradient generators. Both types are covered because the more useful edge template gradient generator is derived from the basic orthogonal gradient generator.

\subsubsection{Orthogonal Gradient Generators}

The detection of the edge maybe found by finding the gradient along a line normal to the slope of the edge. The direction of the slope with respect to the horizontal is $\theta$. Fig. 5.3 shows how the gradient of the direction $\theta$ is found.

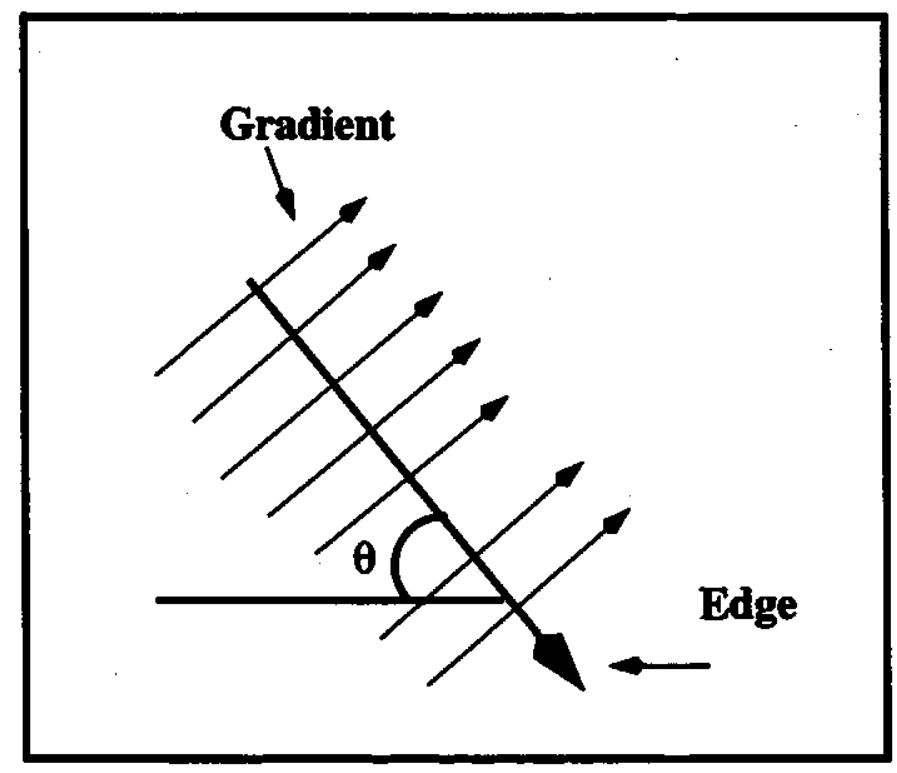

Figure 5.3 The Gradient of a Edge 
The general calculation of the gradient $G(i, j, \theta)$ is

$$
G(i, j, \theta)=\left(\frac{\partial F(i, j)}{\partial i} \cos (\theta), \frac{\partial F(i, j)}{\partial j} \sin (\theta)\right)
$$

Where $\frac{\partial F(i, j)}{\partial i}$ and $\frac{\partial F(i, j)}{\partial j}$ are the gradients of the edges in the $\mathrm{x}$ and $\mathrm{y}$ directions.

This gradient is referred to as the orthogonal gradient, in which two gradients $G_{c}$ column gradient and $G_{r}$ row gradient are orthogonal. By combining theses gradients, the magnitude is found to be

$$
G(j, k)=\left[\left[G_{r}(j, k)\right]^{2}+\left[G_{c}(j, k)\right]^{2}\right]^{\frac{1}{2}}
$$

Or as graphically shown in Fig. 5.4.

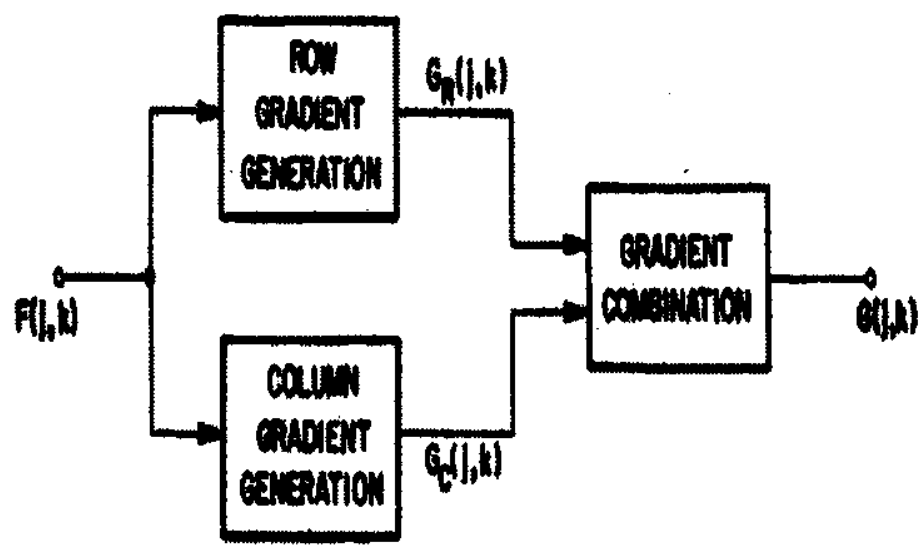

Figure 5.4 The Combination of the Orthogonal Gradients

The direction of the gradient with respect to the row gradient is found by

$$
\theta(j, k)=\tan ^{-1} \frac{G_{c}(j, k)}{G_{r}(j, k)}
$$


The most basic form of an orthogonal gradient filter is the pixel difference filter. $G_{c}$ is the gradient $G(x, y, \theta)$ when $\theta$ equals $90^{\circ}$, and $G_{r}$ is $G(x, y, \theta)$ when $\theta$ equal to $0^{\circ} . G_{c}$ and $G_{r}$ can be found from the horizontal and vertical differences between adjacent pixels, respectively. In other words, $G_{c}$ is the difference between adjacent row pixels, and $G_{r}$ is the difference between adjacent column pixels.

$$
\begin{aligned}
& G_{r}(j, k)=F(j, k)-F(j, k-1) \\
& G_{r}(j, k)=F(j, k)-F(j+1, k)
\end{aligned}
$$

\subsection{5 $3 \times 3$ Gradient Generators}

In the previous section, the orthogonal gradient method was discussed as a simple method for edge detection. In this section, the orthogonal method is carried further by applying this method in the creation of $3 \times 3$ gradient matrices. The adaptation of the orthogonal gradient to $3 \times 3$ gradient was found by Prewitt [6]. This method is the base for three groups of generators, Prewitt, Sobel, and Frei-Chan.

Again the two orthogonal gradients $G_{r}$ and $G_{c}$ are required. The gradients this time are each $3 \times 3$ matrices related by the matrix in Fig. 5.5. Calculation of the row and column gradients are found by applying equations Eq. 5.3 and Eq. 5.4 to the $3 \times 3$ matrix.

\begin{tabular}{|c|c|c|}
\hline$A_{0}$ & $A_{1}$ & $A_{2}$ \\
\hline$A_{7}$ & $G(j, k)$ & $A_{3}$ \\
\hline$A_{6}$ & $A_{5}$ & $A_{4}$ \\
\hline
\end{tabular}

Figure 5.5 The Prewitt Matrix 
The equations that represent $G_{r}$ and $G_{c}$ are

$$
\begin{aligned}
& G_{r}(j, k)=\frac{1}{K+2}\left[\left(A_{2}+K A_{3}+A_{4}\right)-\left(A_{0}+K A_{7}+A_{6}\right)\right] \\
& G_{c}(j, k)=\frac{1}{K+2}\left[\left(A_{0}+K A_{1}+A_{2}\right)-\left(A_{6}+K A_{5}+A_{4}\right)\right]
\end{aligned}
$$

The three groups of generators are only different by the chosen constant $\mathrm{K}$. The constants for each method are

Group K

Prewitt 1 (mathematically consistent with the definition)

Sobel 2

Frei-Chen $\sqrt{2}$

The constants $A_{0}$ through $A_{7}$ and $\mathrm{K}$ determine the gradient direction as North, South, East, or West. The project choose the Sobel method because it smoothes the edges of $y$-direction before calculating the $x$-directional gradient or visa-versa.

The sobel matrices are

Operator Row Gradient Column Gradient

Pixel Difference $\left[\begin{array}{rrr}0 & 0 & 0 \\ 0 & 1 & -1 \\ 0 & 0 & 0\end{array}\right]\left[\begin{array}{rrr}0 & -1 & 0 \\ 0 & 1 & 0 \\ 0 & 0 & 0\end{array}\right]$

Sobel $\quad \frac{1}{4}\left[\begin{array}{rrr}1 & 0 & -1 \\ 2 & 0 & -2 \\ 1 & 0 & -1\end{array}\right] \frac{1}{4}\left[\begin{array}{rrr}-1 & -2 & -1 \\ 0 & 0 & 0 \\ 1 & 2 & 1\end{array}\right]$ 


\subsubsection{Edge Template Gradient Generation}

In the previous section the edge direction was found by the calculation of the orthogonal gradients $G_{c}$ and $G_{r}$, which was then extended to the creation of $3 \times 3$ generators. The Edge Template Gradient generator expands the orthogonal gradient idea to other possible directions. The template filters are preset matrices $\left(H_{m}\right)$, which are convoluted with the image matrix $\left(F_{i}\right)$.

$$
G_{i}(j, k)=F_{i}(j, k) \circledast H_{m}(j, k)
$$

The simplest filters are the $3 \times 3$ matrices, because of their size only eight possible directions of gradient detection are possible (detection every $4^{\circ}$ ). The larger the matrix dimensions the more directions of edge detection. For example, a $5 \times 5$ template gradient has 12 possible directions (detection every $30^{\circ}$ ). The advantage of a larger matrix will give more degrees of freedom, but at cost of CPU time spent on the performing the task.

The method of gradient detection chosen is a modified version of the Robinson 5-level template gradient. These matrices are show in Table 5.1.The Robinson 5-level is derived from the Sobel method, where the Robinson consists of eight $3 \times 3$ matrices to account for all directions available. The north and east directions of the Robinson 5-level correspond to the Sobel matrices in the previous section.

For the calculation of first derivative edge detection, all eight directions are used. The gradient filters are grouped into two groups of four gradients. The first group is North-East, North-West, South-East, and South-West, and the second NorthEastSouthEast, NorthWest-SouthWest, SouthEast-SouthWest, and NorthWest-NorthEast. The results of the gradient calculations are combined in orthogonal pairs. For example, in the first group the NE template is paired with SW, and NW is paired with $\mathrm{SE}$, then the results of these groups are combined. When the other four directions have been similarly combined, all results are brought together. 
Table 5.1 Robinson 5-Level Filters

Direction Standard Modified

\begin{tabular}{|c|c|c|c|c|c|c|}
\hline \multirow{3}{*}{ East } & 1 & 0 & -1 & 2 & 0 & -2 \\
\hline & 2 & 0 & -2 & 4 & 0 & -4 \\
\hline & 1 & 0 & -1 & 2 & 0 & -2 \\
\hline \multirow{3}{*}{ North East } & 0 & -1 & -2 & 0 & -2 & -4 \\
\hline & 1 & 0 & -1 & 2 & 0 & -2 \\
\hline & 2 & 1 & 0 & 4 & 2 & 0 \\
\hline \multirow{3}{*}{ North } & -1 & -2 & -1 & -1 & -4 & -2 \\
\hline & 0 & 0 & 0 & 0 & 0 & 0 \\
\hline & 1 & 2 & 1 & 2 & 4 & 2 \\
\hline \multirow{3}{*}{ North West } & -2 & -1 & 0 & -4 & -2 & 0 \\
\hline & -1 & 0 & 1 & -2 & 0 & 2 \\
\hline & 0 & 1 & 2 & 0 & 2 & 4 \\
\hline \multirow{3}{*}{ West } & -1 & 0 & 1 & -2 & 0 & 2 \\
\hline & -2 & 0 & 2 & -4 & 0 & 4 \\
\hline & -1 & 0 & 1 & -2 & 0 & 2 \\
\hline \multirow{3}{*}{ South West } & 0 & 1 & 2 & 0 & 2 & 4 \\
\hline & -1 & 0 & 1 & -2 & 0 & 2 \\
\hline & -2 & & 0 & -4 & -2 & 0 \\
\hline \multirow{3}{*}{ South } & 1 & 2 & 1 & 1 & 4 & 2 \\
\hline & 0 & 0 & 0 & 0 & 0 & 0 \\
\hline & -1 & -2 & -1 & -2 & -4 & -2 \\
\hline \multirow{3}{*}{ South East } & 2 & 1 & 0 & 4 & 2 & 0 \\
\hline & 1 & 0 & -1 & 2 & 0 & -2 \\
\hline & 0 & -1 & -2 & 0 & -2 & -4 \\
\hline
\end{tabular}

Scale Factor 


\subsubsection{2nd Order Derivative Filtering}

The purpose of second order filtering is to enhance the edges of an image. The importance of enhancement is to provide a more continuous edges, rather than broken lines. There are several second order methods available but the Laplacian Generation method is by far the simplest, and fastest since it only uses a single $3 \times 3$ matrix.

\subsubsection{Laplacian Generation}

The Laplacian method is defined as a 2nd order derivative $G(x, y)$ of the input function $F(x, y)$.

$$
G(x, y)=\left(\frac{\partial^{2}}{\partial x^{2}}+\frac{\partial^{2}}{\partial y^{2}}\right) F(x, y)
$$

The response of $G(x, y)$ is zero when the change in $F(x, y)$ is linear. When the rate of change of $F(x, y)$ is no longer linear $G(x, y)$ maybe negative or positive in value. When a transition from positive to negative or negative to positive occurs, an edge is found. This transition is considered as the zero crossing of the second derivative. In a 2-D image, the transitions will occur in pairs thus forming double lines along the edge. The true edge is neither of these but is located between the double lines. For Eq. 5.8, the image data must be read in from left to right, top to bottom. 
There are various adaptations of the Laplacian in matrix form. The objective of the Laplacian matrix is to detect the change between a pixel and it's adjacent directions. To find the impulse response between a pixel and eight neighbors two possible choices are available. The first filter is a Laplacian in which the difference of slopes is averaged over three rows and three columns. To construct this filter the rows and columns can be represented separately.

$$
H=\left[\begin{array}{lll}
-1 & 2 & -1 \\
-1 & 2 & -1 \\
-1 & 2 & -1
\end{array}\right]+\left[\begin{array}{rrr}
-1 & -1 & -1 \\
2 & 2 & 2 \\
-1 & -1 & -1
\end{array}\right]
$$

The normalized filter is then

$$
H=\frac{1}{8}\left[\begin{array}{rrr}
-2 & 1 & -2 \\
1 & 4 & 1 \\
-2 & 1 & -2
\end{array}\right]
$$

The second filter is constructed with the slopes calculated as the difference between the center component and it's eight neighbors.

$$
H=\frac{1}{8}((F(i, j)-F(i-1, j-1))+(F(i, j)-F(i-1, j)) \ldots .)
$$

The matrix representation of Eq. 5.11 is

$$
H=\frac{1}{8}\left[\begin{array}{rrr}
-1 & -1 & -1 \\
-1 & 8 & -1 \\
-1 & -1 & -1
\end{array}\right]
$$


The extraction of the edge information alone requires the sum of the matrix terms to be equal to zero. Therefore, the center element must equal the negative of all directional terms. If a filter were needed to enhance an image's edges then the filter would be the following.

$$
H=\frac{1}{8}\left[\begin{array}{rrr}
-1 & -1 & -1 \\
-1 & 9 & -1 \\
-1 & -1 & -1
\end{array}\right]=\frac{1}{8}\left[\begin{array}{crr}
-1 & -1 & -1 \\
1 & 8 & -1 \\
-1 & -1 & -1
\end{array}\right]+\left[\begin{array}{lll}
0 & 0 & 0 \\
0 & 1 & 0 \\
0 & 0 & 0
\end{array}\right]
$$

The filter shows that when the sum of the filter elements is not equal to zero edge information is added to the image pixels, this is called edge enhancement. Different forms of Laplacian depend on the number of directions required and the extraction of edge information or edge enhancement. For this project, the need for equal weighting in each direction is necessary. Therefore, Eq. 5.12 is best suited for detecting edge information in eight directions 


\subsection{Facial Feature Extraction}

To find the features in the face, it must be understood what important features there are. From birth, the human brain learns to recognize a face from the features of the eyes, mouth, and head. The computer is now to recognize the face from image data without prior learning. It is essential to provide some basic information as to what the face is and impose some restrictions on input facial images. The video clip of Claire is the image database used for testing the methodology of facial feature detection. Developed algorithms include a limited knowledge base. However, it is worth noting that the analysis of Claire does not necessarily work for other pictures. The features required for the facial encoding can be divided into two categories (classes). First, the face has parts that must be found directly from individual image frames. This includes the head, eyes, nose, and mouth. The other is interframe information, which can be obtained by tracking facial features in successive frames to measure head motion for example.

\subsubsection{Average Amplitude Projection}

In image processing, the size of a picture is an important factor that decides the time necessary for applying a particular function or image filter. Any method that can reduce the size by removing unnecessary parts of an image can save time. "Amplitude Projection" is such a method.

Facial part detection is concerned about the head and face of a person. What amplitude projection does is detect the general area of the head from the fluctuation in the average pixel density. Vertical or horizontal lines of pixels are read in one pixel at a time, and the averages of their lines are calculated and then stored in an array. The horizontal and vertical arrays are defined in Eq. 5.14 and Eq. 5.15 for an input image $F(j, k)$. 


$$
\begin{aligned}
& H(k)=\frac{1}{N} \sum_{j=1}^{N} F(j, k) \\
& V(j)=\frac{1}{N} \sum_{k=1}^{N} F(j, k)
\end{aligned}
$$

Somewhere in arrays $H(k)$ and $V(j)$ should show a sudden change in value when an object is found. From the data a general width and length of the object can be estimated. For the image of a person with a monotone background the extraction of the person's head area can easily be done.

In this project, only the vertical process was applied with a limited number of vertical lines checked. The two features searched for were the top of the head and the shoulder locations. The top of the head is where the projection method finds the change in $V(j)$ that is close to the top of the image. In finding the shoulders, the first sudden vertical change in projection value is looked for as the search moves away from the top of the head. By finding locations of the one or two shoulders and the top of the head, a box confining the head can be formed. The box defines the section of the image for edge detection. This method only performs a general head extraction, so a larger boundary should be configured into the size of the box. 


\subsection{Directly Extractable Features}

\subsubsection{Application of Filters for Edge Detection}

This section covers the important features found as it pertains to the database Claire. The information found from the database is general enough to be translated to other faces. By applying the edge detection filters to a picture of a person the important features become apparent. However, the valid edge information must be selected using a threshold value. This value is chosen from the image's luminance or gray scale information. For Claire, the average pixel value in the head region works best as the threshold value. This may be different for other images.

The extraction of edge information is accomplished by applying the first order or second order derivative filters. The first order filters are the modified Robinson 5 Level filters. The minimum number of directions is four, but the need for solid edges requires all eight. The preferred second order filter is the Laplacian in Eq. 5.12. The choice of filter should closely resembles Fig. 5.6 after it is applied. The Robinson best fits this requirement, however the Laplacian may be valid in the future if double edges can be over come. In a typical edge detected frame, features commonly found are shown in Fig. 5.6. The eyes, eyebrows, nostrils, mouth, and head edges are ideal when each is clear and separate.

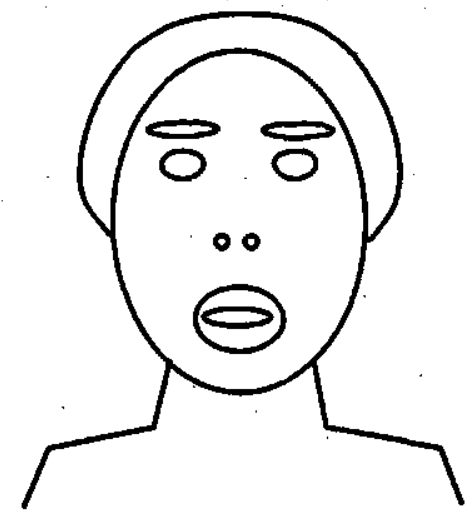

Figure 5.6 The Ideal Edge Detected Image 
The head edges must be distinguished as inner and outer edges. The outer edge is important in head rotation measurements, and the inner is in the detection of the other features. The outside edge can be found by searching the edge-detected image from the outside inward on both sides. Finding the inside edge will be described in the next section. Whether both edges coincide depends on factors like hairstyle.

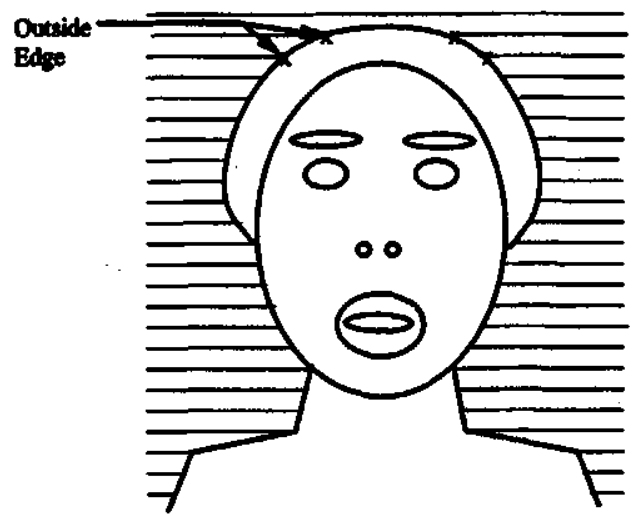

Figure 5.7 The Outline of the Head

The outside edge is the easiest feature to find in an ideal image. It just requires the first and last edge pixel on each horizontal line. The ease of extraction not only depends on the edge detection filtering, but also the difference between the background luminance and the luminance of the person. The ideal situation occurs when the background is a distinct uniform gray level from the head. In images with a lack of distinct gray levels miscellaneous edges will appear. The reason for these problems is the high frequency sensitivity of the gradient and Laplacian filters, and the choice of threshold. If the background is no longer ideal, but contains patterns or objects, the filters will find unwanted edges.

The implementation of the outer edge of the head will be discussed in later sections of this chapter. 


\subsubsection{Finding the Objects}

The extraction of each eye and the mouth requires the analysis of the contents inside the head outline. Features from an edge-detected head can best be found by the general assumption that inside the face contains many distinct objects. Initially, the computer does not know which objects are the eyes, nose, mouth, or invalid objects. The first step is to extract all the objects and sort them later. The following is a list of the subsections that explain how to find the objects in the face.

\section{Vertical Split Line}

2. Sweeping of the Face

3. Multiple Sweeps

4. Finding the Inner Edge of the Head

5. Object Extraction

\section{Vertical Split Line}

This is the division of the head along a vertical line starting at the top of the head. Dividing the head into two halves allows the analysis inside the head to find the inside edge. If an ideal split occurs the line would hit the mouth and not the eyes. If this is the case two more assumptions can be made. The first assumption is the whole left eye is in the left half of the head, and the whole right eye is in the right half. The second assumption is the mouth is in both sections and the vertical line split intersects the mouth. An example of the vertical line division is shown in Fig. 5.8, where the vertical line at the position one is the vertical line split.

\section{Sweep of the Face}

To find the inside edge of the head the face must be searched using the vertical split line and the outside edge of the head as boundaries. Sweeping consists of performing a pixel by pixel horizontal line search for edge pixels. The search in the right side is 
done by sweeping from the vertical split line to the outside edge moving downward from the top to bottom. This process is repeated to sweep the left side of the face. An edge is the change in pixel intensity as the edge-detected image will only contain two values.

In using the method of sweeping, a typical outcome is shown in Fig. 5.8. The shaded area represents the region the face searched when starting from position one. This search is only capable of finding the small central portion of the inside edge of the head. Therefore, the sweep method must be expanded to a more detailed search.

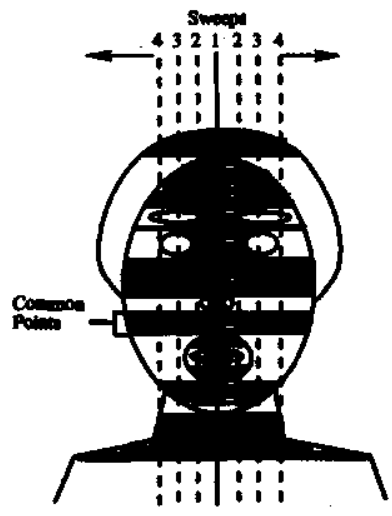

Figure 5.8 First Sweep of the Face

\section{Multiple Sweeps}

To improve the sweeping method multiple sweeps can be made to find inner edge points. The purpose of multiple sweepings is to sweep at a different vertical line position. Changing location of the sweeps is done by moving the vertical split line outward by a set interval so the sweep can move beyond objects and reach the inner edge. Fig. 5.8 shows the three other possible sweep starting positions two, three, and four; the right half and the left half use separate lines but are at equal distance from position one. Every different sweep performed creates one complete set of edge information of the inside of the face. For the Claire's image, the search works best when the sweeps are placed every 4 pixels starting at the middle with position one and continues outward with 12 other positions. After each sweep the extracted edge information must be stored. The data found from the sweeps gives a simple map 
inside the face.

Find the Inner Edge of the Head

The sets of the extracted data contain the locations of the inner edge for the left and right halves. What must be done now is to sort the data to find the correct edge. The difficulty in using the data is the inner edge oval shape tapers sharply at the top and bottom. Three steps have been implemented to find as many usable edges as possible. These steps are:
i. Common Edges
ii. Mouth and Nose Edge Search
iii. Eyes and Eyebrows Edge Search

These steps are not limited to a specific part of the sets, but are applied to the whole data set. This means that steps $i i$ and $i i i$ are not fixed to these regions.

\section{i. Common Edges}

The first step in inner edge extraction is to compare all sets to find edges that do not change when the sweep position moves. These points can be assumed to be on the inner edge; Fig. 5.8 shows points common to all sets. The remaining edge points can be divided into two types, objects on the vertical splitting line, and objects off the vertical splitting line. Each of these types are handled in steps $i i$ and $i i i$ respectively. 


\section{ii. Mouth and Nose Edge Search}

The width of the multiple sweeps was specifically made wide to sweep past the eyes and eyebrows. Since the mouth and nose are centered to the middle of the face, the most common edge information found in this region must be on the inner edge. From every sweep set, the data will have two points per horizontal scan line. By comparing all the sets that cross a particular scan line in the nose and mouth region, the most common edge should be the inner edge. To confirm the new inner edges they can be compared to inner edges found in step $i$.

\section{iii. Eyes and Eyebrows Edge Search}

The inner edge and the eye and eyebrow features, are usually close together, therefore identifying the edge require a more rigorous search. Two possible methods to solve this problem have been considered.

The first method uses a variation of the sweep method. For lines where the inner edge is not identified, the furthest position on the scan line (as found by the multiple sweep method) is compared to a known adjacent inner edge. If these two edges are far apart ( $>5$ pixels) then the unknown edge's line should be swept again starting from the edge furthest out and swept inward. Otherwise, the position furthest out is adequate for the inner edge.

The second method requires detection of unknown edges by reading the data set sequentially starting with an identified inner edge next to an unknown edge. The assumption is the position of the known edge must be very close to the unknown edge. Therefore, the closest edge pixel on the unknown line to the known edge is the correct inner edge. As each new edge position is found it is added to the group of inner edge positions and is used to find other edges. 


\section{Object Extraction}

With the inner edge defined, it is known that all facial features are contained within. The features in the face at this point can be considered as objects since it is uncertain about their identity. To find the objects the sweep method is repeated with just two sweeps. In the first sweep, or outward sweep, the starting position is the vertical split line as shown in Fig. 5.9(a). If the horizontal line scans outward and finds an object then this position is recorded, otherwise the scan line is ignored. The second sweep, or inward sweep, starts from the inner edge and sweeps inward to the vertical scan line. The inner edge is split into two sections the right half and left half as dictated by the vertical split line. As the horizontal line sweeps inward, the outside edge of an object or the vertical split line is found. Fig. 5.9(b) shows the inward sweep that would occur. When the vertical split line is encountered that line would be ignored.

The results of the two sweeps can be combined to find the outline of the objects. In an ideal case Fig. 5.9(c) shows the six objects that can be identified using edge information found from Fig. 5.9(a), and Fig. 5.9(b). However, if the conditions of the object extraction are not ideal two common problems can occur, extraction of unwanted objects, and merging of objects. Since problems like these can occur it is not always possible to identify the features by the logic described here. 

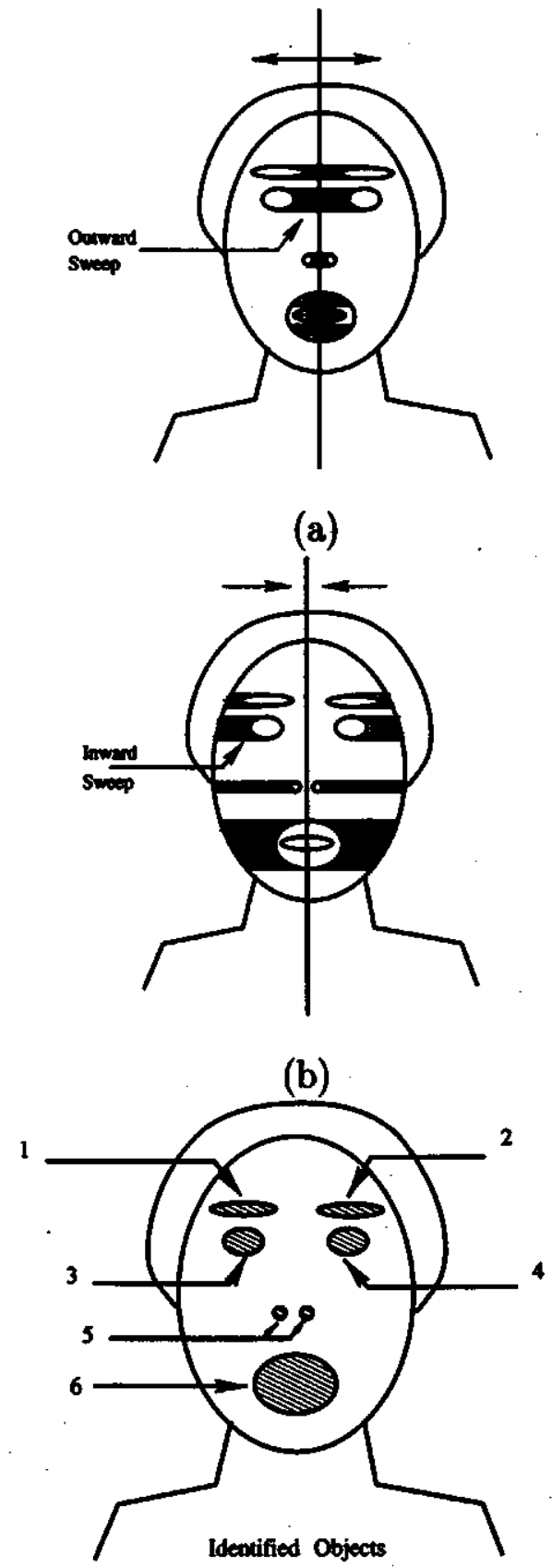

(c)

Figure 5.9 The Combination of the Inner and Outer Sweeps to find the Objects 


\subsubsection{Object Identification}

To a person observing the locations of the objects for a face, it is simple to identify them. For the computer, reference information must be given on how the objects in the face are structured. Ideally, to do a proper job in object identification technology such as Neural Networks is required for learning face patterns. Due to the wide scope of work already covered in this project, it was decided to limit object extraction to a simple analysis on the relationship of objects with respect to each other.

The determination of the correct objects can be based on object location and size. However, object size heavily depends on good object extraction. Due to the uncertainly of the extraction process object identification will only concern location information. The importance of extracting the correct facial components is based on the relative object placement. The position of the eyes, nose, and mouth compared with each other is a well-defined parameter. The observations of a face looking forward can be categorized by the following.

a. The eyes align horizontally with each other.

b. The nose is located between the eyes.

c. The mouth is located between the eyes.

d. The mouth and nose align vertically.

e. The nose is between the mouth and eyes.

These observations suggest that eyes, nose, and mouth form a $Y$ pattern using feature centroids, where the word centroid is referred to the center of an object. In object identification, some objects such as the mouth may have formed one or two objects, therefore more centroids may appear. For a simple example of the facial objects, a group of ten pictures from Claire's database were processed with the object extraction methods. All centroids from the ten frames are superimposed upon each other. Fig. 5.10 shows the scatter plot of these groupings. 


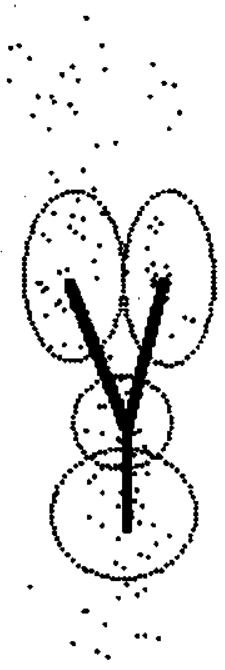

Figure 5.10 Scatter plot of Centroids from frames 0 to 9

The circled regions in Fig. 5.10 represent the groups for the right and left eyes, nose, and mouth. These four groups form a distinctive $Y$ pattern. The centroid movement from one frame to the next frame is small, therefore tracking movement of the four features frame to frame is possible. The frame to frame relationship can be considered an adaptive process. 


\subsection{Interframe Extractable Features}

\subsubsection{Adaptive Object Extraction}

Adaptive object extraction uses the known facial structure and past frame information to quickly identify facial objects. The adaptive extraction is specifically for the four base objects described in the previous section. Since the centroid is abstract information resulting from feature extraction, some risks are always involved about whether the adaptive scheme properly works. The problems with adaptive methods can be ranked by risk. The lowest risk is trusting tolerances set for interframe object movement. The next is the assumption that order of identified objects is consistent. What is meant by order is the sequence that the four basic objects appear is the $Y$ pattern. The third risk is assuming the identified objects in a previous frame are correct because these objects are used as a reference in the current frame. These three risks must be accepted to use adaptive methods for interframe feature extraction.

In section 5.3.2, a vertical line was used to divide the face into two halves so the inside of the face can be swept. However, this assumption is valid for a face positioned perfectly upright. If a vertical line always divides a frame, when the head tilts too much the eyes can not be separated by the vertical line. The object extraction therefore will fail. Using adaptive methods would improve the placement of the split by using the line that takes advantage of the four base object found in the previous frame. The split occurs in two line segments, the first is a line from the top of the head to the nose, and the second is a line from the nose to the bottom of the face. The first line is created by using the coordinates of the nose, and the middle point between the eyes. The second line uses the centroid from the nose and mouth. Then the maximum width of the right half and left half must be found to adjust the number of sweeps made on each side. The methods of sweeping will remain the same as shown in Fig. 5.12. The procedure requires that the objects must always be correctly identified. 


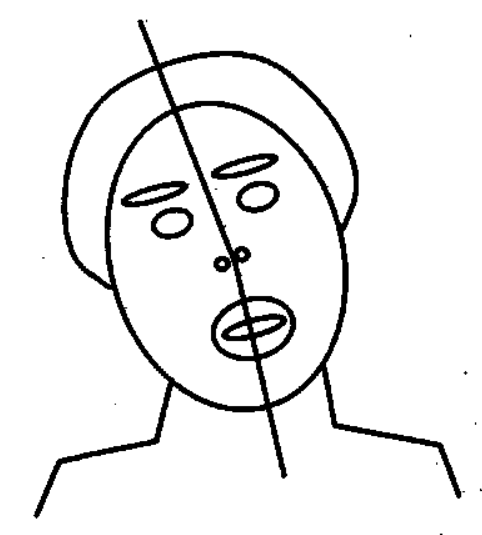

Figure 5.11 A Typical Adaptive Head Split

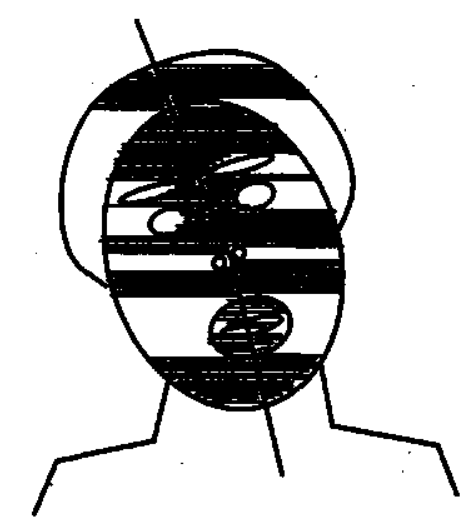

Figure 5.12 Sweep Pattern for the Tilted Head 


\subsubsection{Rotational Extraction}

The fastest way to find rotation of a head is by the relative movement between facial objects, and the outer and inner edges of the head. The three directions of motion are in terms of the $\mathrm{X}, \mathrm{Y}$, and $\mathrm{Z}$ coordinates. Where $\mathrm{X}$ and $\mathrm{Y}$ represents the 2-D image plane and $\mathrm{Z}$ represents the depth.

The difficulty lies in finding 3-D head rotation from 2-D information. To understand the relationship between a 2-D image and a 3-D object must be viewed from the perspective of a camera. The process of taking a picture requires three things, the image plane, the lens, and the 3-D object (point), as shown in Fig. 5.13 [6]. The translation of a 3-D point $(X, Y, Z)$ to a 2-D surface at point $(x, y)$ is dependent on the focal length $F$ of the lens.

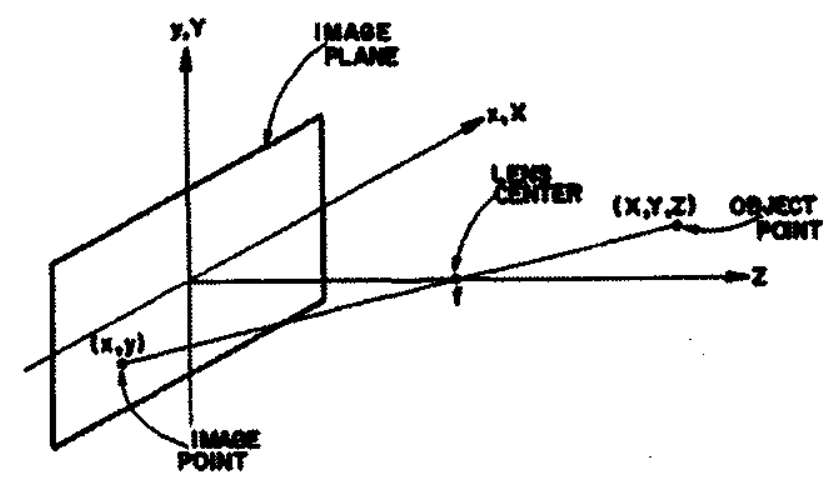

Figure 5.13 Relationship between 3-D and 2-D

By similar triangles the relationship of the 2-D point from the 3-D point is

$$
\begin{gathered}
x=\frac{F X}{F-Z} \text { and } y=\frac{F Y}{F-Z} \\
X=\frac{x}{F}(F-Z) \text { and } Y=\frac{y}{F}(F-Z)
\end{gathered}
$$

However finding the exact 3-D point from the single 2-D point is not possible, simply because the depth $\mathrm{Z}$ is unknown. It is only possible to solve for 3-D coordinates if this information about $\mathrm{Z}$ is given. 
To achieve exact 3-D positioning for 2-D images requires the use of two or more cameras. Without using the mathematical model described, an image from one camera can still tell a significant amount of information about the movement patterns of facial objects. The four base objects are the focus of the movement. Any horizontal movement of the $\mathrm{Y}$ pattern can be attributed to as movement in the $\mathrm{X}-\mathrm{Z}$ or $\mathrm{X}-\mathrm{Y}$ planes. Any vertical movement in the pattern causes movement in the X-Z and Y-Z planes. Movement of the $\mathrm{Y}$ pattern with respect to the inner or outer edge of the face is also a very good indicator of $X-Z$ and $Y-Z$ motion. Since $X-Y$ motion relates best to the image it is always extracted first, then $\mathrm{X}-\mathrm{Z}$ and $\mathrm{Y}-\mathrm{Z}$. Some observations about how to distinguish the differences between the planes, are made as follows.

\section{X-Y Rotation:}

Rotation in this plane is shown by the consistency of the distances between the four base objects in an image sequence. As well, all features should rotate with respect to a pivot.

\section{X-Z Rotation:}

The distance between the inner edge of the face and the facial objects should change with respect to the $\mathrm{X}$ plane. The distance between the facial object and one side of the inner edge should increase, while the distance of the same object and the other inner edge should decrease. For example, the distance between the nose and the inner edge should show a decrease on the left and increase on the right as the head turns left.

\section{Y-Z Rotation:}

The rotation is for simulating nodding of the head. The change in vertical distance between the four objects should indicate this type of rotation. Distance is largest when looking directly at the camera and decrease as the head vertically rotates. When choosing good objects for measuring this rotation, the further the objects are from the center of the face the more movement can be observed. 


\subsubsection{Using Pattern Recognition for X-Y Plane Motion}

Pattern Recognition of objects in a 2-D surface is the comparison of a known template to a surface containing the same pattern. The ability to use pattern matching to detect an object moving in three dimensions using a 2-D perspective is limited. This is because pattern matching cannot account for any $\mathrm{Z}$ plane motion. The procedure for performing the match is by comparing overlap of the two patterns. The two patterns chosen for comparison are the outer edge of the reference frame, and the outer edge detected in subsequent frames. To improve the overlapping of the two edges the algorithms, rotation, displacement, and scaling are used to manipulate the reference frame's position. The order that these algorithms are applied and the number of iterations used must be carefully controlled.

As an example, two images, the sample edge and the reference frame edge are compared in Fig. 5.14. The two patterns are matched by calculation of the minimum overlap error. Each motion is tried in the sequence of rotation, displacement, and scaling (not shown), to reduce the error between the reference and the sample frame. Fig. 5.14 shows only the first iteration applied. Successive iterations can improve the match even further, but repeating the sequence will increase operation time.

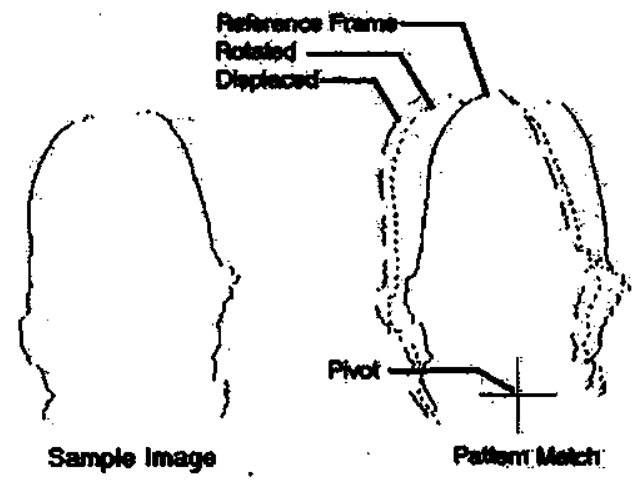

Figure 5.14 Example of Pattern Matching 


\section{Experimentation on the Encoder and Decoder}

\subsection{Introduction}

The purpose of the theoretical work is to develop the encoder/decoder sections for a video telephone application. In the video telephone, data requirements of the receiver must come in two forms, overhead information, and individual frame information. Overhead information covers data common to individual frames such as the reference image, and the warping grid. The individual frame information involves parameters that describe the movement in the face from frame to frame. This must be sent continuously once the system is initialized. Experimentation focuses on the use of individual frame parameters that correspond to the encoder/decoder algorithms.

This chapter is divided into two sections, decoder testing and encoder testing. The decoder will be tested from two aspects. The first is the testing of each mesh movement algorithms described in chapter 4 . The second aspect is to prove the use of the mesh algorithms with the bilinear warping algorithm to verify the ability to simulate the facial movements. Encoder tests cover the application of the facial detection algorithms and the interpretation of the measured facial parameters in terms of the motions required by the decoder. 
The work done on encoder and decoder algorithms was completed at a software level. The computer system used is an IBM compatible Pentium $200 \mathrm{Mhz}$ system. The use of a fast computer gives the advantage of quick frame by frame image simulation and detection. The capability of using a Pentium processor is comparable to current workstations available. However, the PC is more versatile and less expensive. The programming language used is Borland's $4.5 \mathrm{C}++$ operating with 32 bit structures for Windows and DOS. The advantage of the package is the user-friendly graphical capabilities.

\subsection{Functional Tests for the Decoder}

The purpose of the decoder is to take facial movement parameters and translate these parameters into mesh motion. To properly test the algorithms, general operations are looked at first. Then the mesh routines are tested for ability to simulate facial movements using the bilinear image warping.

\subsubsection{Attractor Repellor}

To verify the application of the Attractor/Repellor, two tests have been chosen to show control capabilities.

\section{Test 1: Control variables $\alpha$ and $n$}

The simplest case is the use of one grid node and one mass node. The terms that control the movements are the gain control parameter $\alpha$, and the range control parameter $n$, as shown in Eq. 6.1. $\mathrm{r}$ represents the initial vector between the mass node and the grid node, as in Fig. 6.1. $\mathbf{r}^{\prime}$ is the resulting vector from the application of the Attractor/Repellor. Instead of testing both the attractor and repellor, this test will only involve repellor action. The difference in using the attractor is the sign of $\alpha$ must be negative.

$$
\mathbf{r}^{\prime}=\mathbf{r}\left(1+\frac{\alpha}{\left|\mathbf{r}^{n}\right|}\right)
$$




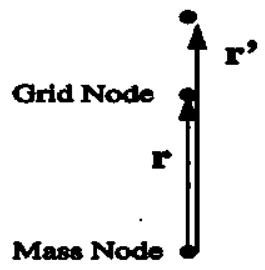

Figure 6.1 Test 1: 1 Grid and 1 Mass Node

To test the movement of the nodes, $\alpha$ and $n$ are each tested as a variable. In Fig. 6.2, Eq. 6.1 is represented in the plot of $\mathbf{r}$ versus $\mathbf{r}^{\prime} . n$ is tested for values two and three. For each case, $\alpha$ increases from zero to two in 0.5 increments. Each curve represents the movement of the grid node over a range of possible $r$ values. The section of the curve where $\mathbf{r}^{\prime}$ increases with $\mathbf{r}$ is the function of the $A / R$. The range zero to one for $\mathbf{r}$ is omitted because values of $\mathbf{r}^{\prime}$ approach infinity as $\mathbf{r}$ decreases.

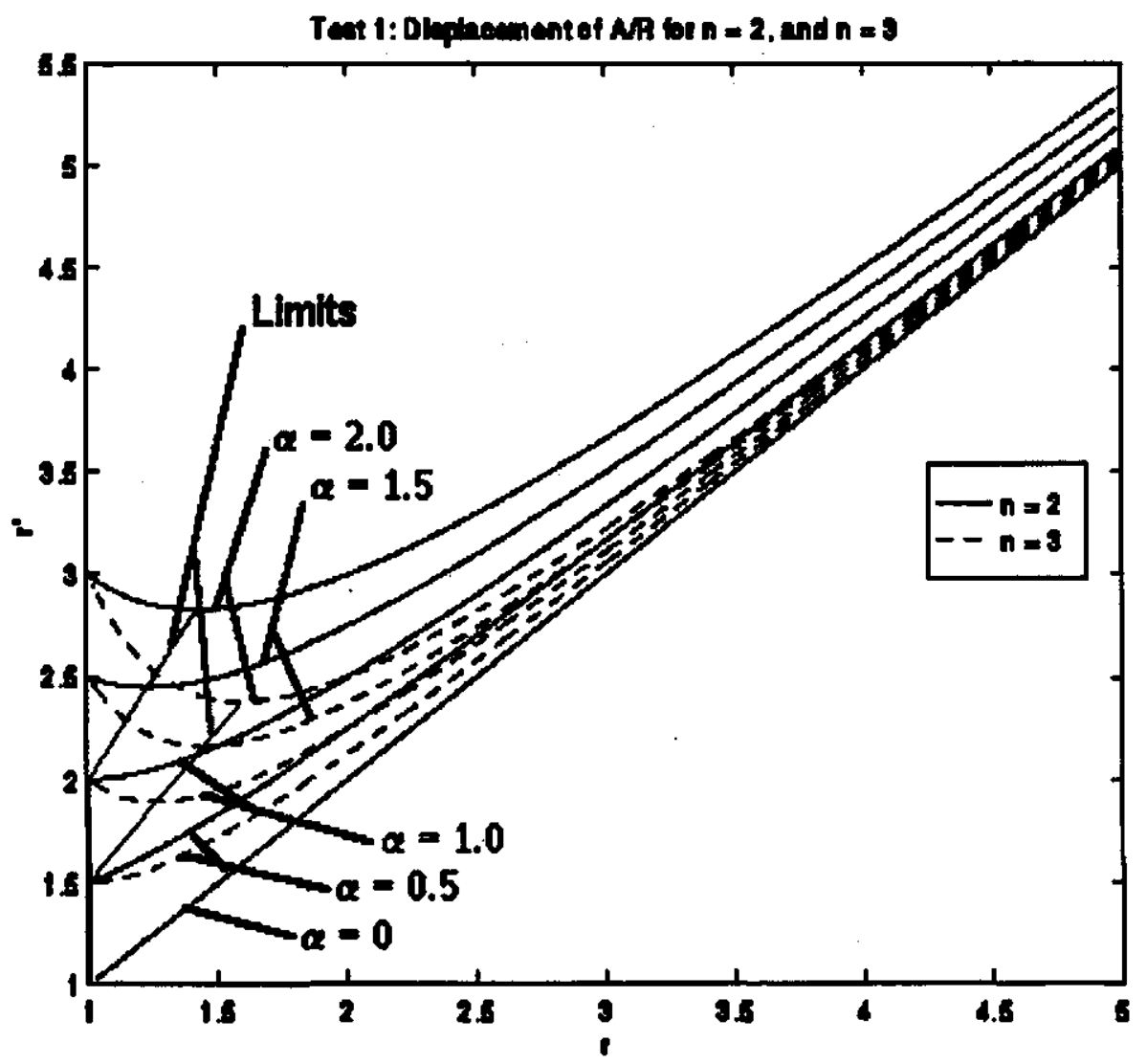

Figure 6.2 Movement of Grid Nodes using $n$ and $\alpha$

The intended use of variable $n$ is for controlling the range of motion of a grid node. In Fig 6.2 the curves for $n$ equal to two cover a much broader range of movement 
than $n$ equal to three. Therefore, when $n$ is large the amount of distance added (or subtracted for the attractor) is less. This is should be expected since in Eq. $6.1 n$ is the power of the inverse distance $r$.

$\alpha$ is a gain factor of the Attractor/Repellor. The purpose of $\alpha$ is to control over the location in which the grid node can move. By changing $\alpha$, a curve more suited for the desired grid motion can be selected. Fig. 6.2 shows five examples of $\alpha$, as $\alpha$ increases starting from zero (no grid movement) the movement of a grid node increases. However, the amount of movement achieved by $\alpha$ is limited. The limits for $n$ equal to two and three are shown in Fig. 6.2 as two lines intersecting the minimum values of $r^{\prime}$ for each curve. If values to the left of this line are chosen then the movement will no longer be the expected action of the A/R. At values below this limit grid node crossover will occur.

To define the occurrence of crossover, two vectors, $\mathbf{r}_{0}$ and $\mathbf{r}_{1}$, are represented in Fig. 6.3. The condition for the point $X_{0}$ following $X_{1}$ when the $\mathrm{A} / \mathrm{R}$ is applied is defined by the inequality Eq. 6.2 .

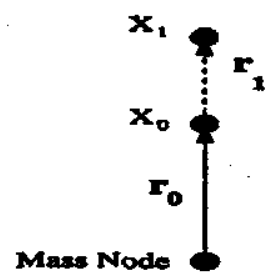

Figure 6.3 Test 1: 2 Grid nodes and 1 Mass Node

$$
\left|r_{0}\left(1+\alpha \frac{1}{\left|r_{0}\right|^{n}}\right)\right|<\left|r_{1}\left(1+\alpha \frac{1}{\left|r_{1}\right|^{n}}\right)\right|
$$

Since $\mathbf{r}_{0}$ and $\mathbf{r}_{1}$ are aligned in the same direction these vector can be represented by scalar values $r_{0}$ and $r_{1}$.

$$
\left|r_{0}\left(1+\alpha \frac{1}{\left|r_{0}\right|^{n}}\right)\right|<\left|r_{1}\left(1+\alpha \frac{1}{\left|r_{1}\right|^{n}}\right)\right|
$$


If Eq. 6.3 is written in terms of $\alpha$

$$
\alpha<\frac{r_{0}^{n-1} r_{1}^{n}-r_{0}^{n} r_{1}^{n-1}}{r_{1}^{n-1}-r_{0}^{n-1}}
$$

This inequality gives a guideline to the limit of $\alpha$ such that point $X_{0}$ does not move further than $X_{1}$. For the purposes of facial motion a small $n$ is preferred, so that $\alpha$ is used as the control for facial motion. The useful range of $n$ for enhanced warping effects is between 1.0 and 2.5 .

\section{Test 2: Effects of Multiple Mass points on the shape of a Mesh}

The movement of mouth and eyes is the primary use of the A/R. The measurement of the mouth and eye dimensions should be made at the maximum width, in the middle of the features. To simulate these actions most of the movements may be considered symmetrical. The eyes movements are strictly vertical motions, and the mouth uses both vertical and horizontal movement. To control the movement of the grid nodes, the number of mass nodes and their placement must be observed.

The simplest situation is with the use of one mass node. The test grid places eight grid nodes arranged in a square. In Fig. 6.4, the mesh is tested for a repellor situation with the mass node placed at the center of the eight grid nodes. Since the grid nodes are symmetrically placed about the mass node, all grid nodes move equally in all directions, as shown by the repelled grid. One mass node produces a movement in two directions, horizontal and vertical. The magnitude of movement in each direction can be separately controlled by setting different values of $\alpha$ in Eq. 6.4 for horizontal/vertical pair as $(\alpha, \beta)$. However, the mass node cannot be moved if complete symmetry needs to be maintained. To verify this, the node is moved to the right one quarter length of the square. Fig. 6.5 shows the result of displacing the mass point. Grid nodes of the right side are displaced more than the left. This grid shape does not define a simple controllable action. 


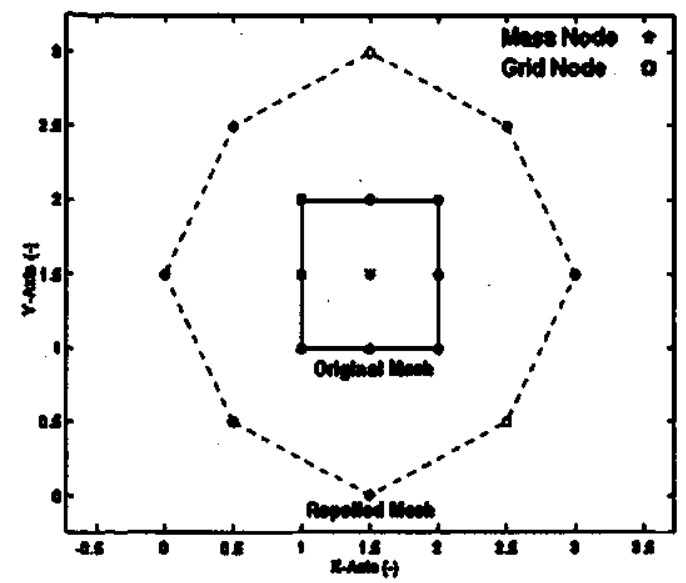

Figure 6.4 Symmetrical Repellor action for 1 Mass Node

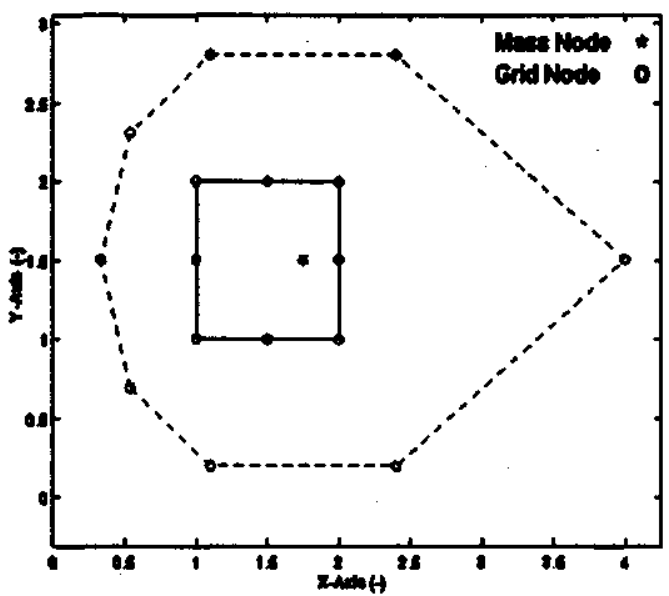

Figure 6.5 Horizontal Symmetrical action for 1 Mass Node

In the case of two mass nodes, movement of grid nodes can be directional. Fig. 6.6 and Fig. 6.7 show the movement of the mesh in a horizontal and vertical direction. The placement of the grid nodes requires that the two masses be of equal distance from the center of the mesh and lie on the same line as the center of the mesh. By moving the nodes further from the center the directional effect is increased. The closer the nodes are to the center the more the result approximates the single node case. 
For the eyes, a mass node pattern in Fig. 6.7 is best suited. The mouth requires the use of four mass nodes, which resembles the combination of the node positions in both Fig. 6.6 and Fig. 6.7 .

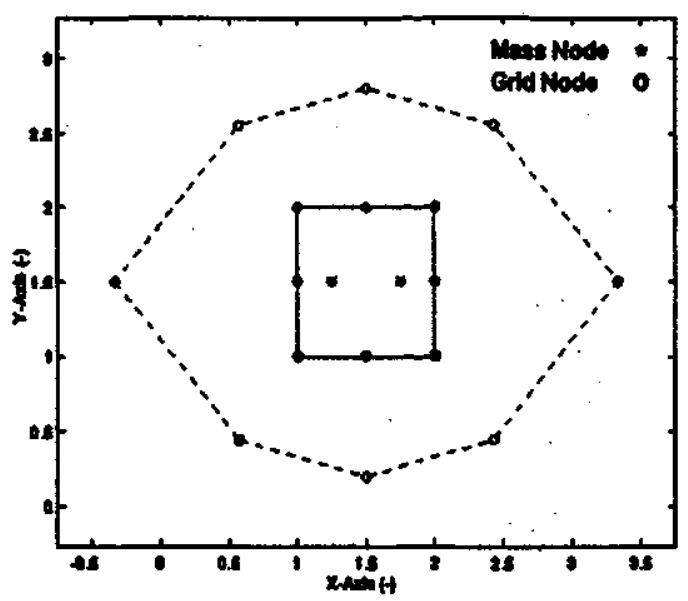

Figure 6.6 Enhanced Horizontal Movement, Two Mass nodes

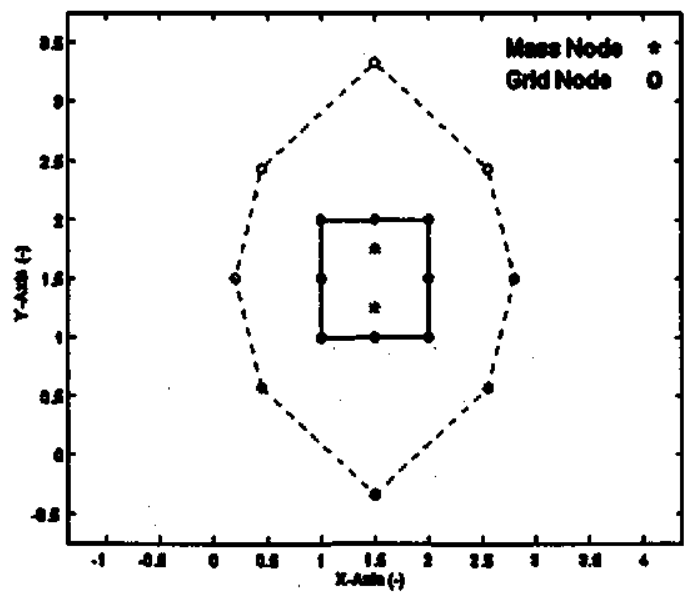

Figure 6.7 Enhanced Vertical Movement, Two Mass nodes 


\subsection{2 $\mathrm{X}-\mathrm{Z}$ and $\mathrm{Y}-\mathrm{Z}$ Rotation}

In chapter 4 the rotation of the head in each direction is described by Eq. 4.12. $\mathrm{X}-\mathrm{Z}$ rotation represents $\mathrm{x}$-coordinate movement of the grid nodes, and $\mathrm{Y}-\mathrm{Z}$ rotation is y-coordinate movement of the grid nodes. As an example of $\mathrm{Y}-\mathrm{Z}$ motion, Fig. 6.8 is the location of grid nodes across the head block. $y_{1}$ and $y_{0}$ are the boundary values of the head, and $y_{i}$ is the current position of the reference point in the face. Rotation of the head causes $y_{i}$ to move to the new position $y_{i}^{\prime} . n$ is the degree of distortion in the grid, for the purposes of testing both second order $(n=2)$ and third order $(n=3)$ will be shown. $a$ describes the amount of rotation that occurs, the choice of $a$ is found by writing Eq. 4.12 in terms the reference positions $y_{i}$ and $y_{i}^{\prime}$.

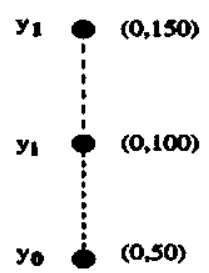

Figure 6.8 Basic Position of Head Rotation Parameters

To understand how 3-D rotation is achieved using a 2-D image, the mesh is viewed from the perspective of directly facing the camera. When the reference point moves from $y_{i}$ to $y_{i}^{\prime}$ one side of the mesh will compress and the other will expand. The compressed side of the grid gives the appearance of features moving away from the camera. If the rotation is significant, features that were at the center of the face will overlap a side of the face. The side that expands will produce the opposite effect by the movement of its features toward the center of the face.

For a demonstration of the mesh movement the values of $y_{0}, y_{i}$, and $y_{1}$ are set to 50,100 , and 150 respectively. $y_{i}^{\prime}$ and $a$ are related by Eq. 4.12. The relative displacement of $y_{i}^{\prime}$ when $y_{i}$ moves from from 50 to 150 is shown in Fig. 6.9(a) and Fig. 6.9(b) for five different values of $\alpha$. Fig 6.9(a) is for $n=2$ whereas Fig 6.9(b) is for $n=3$. The graphs suggest that $y_{i}^{\prime}$ is greater than $y_{i}$ when $a$ is negative, and $y_{i}^{\prime}$ is less then $y_{i}$ when $a$ is positive. When $a$ is zero, there is no movement of $y_{i}$. 


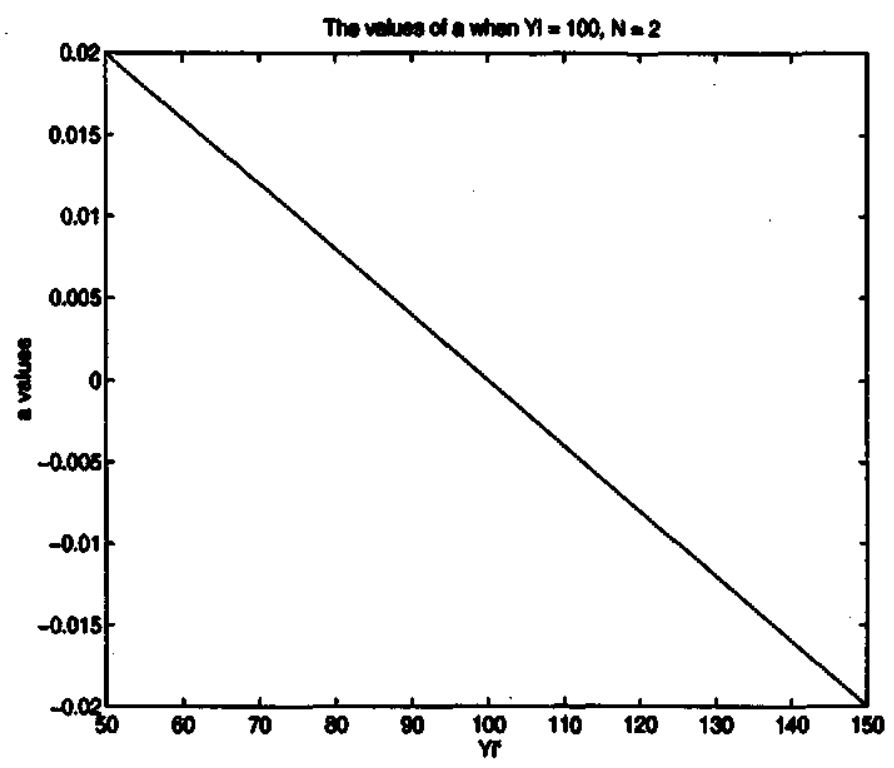

(a)

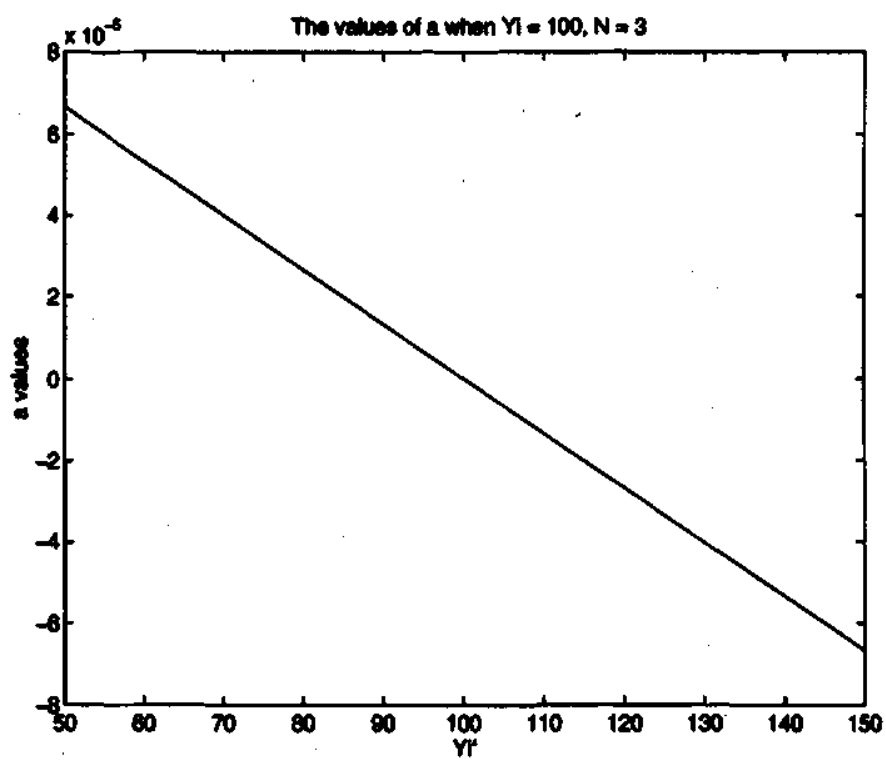

(b)

Figure 6.9 Testing $a$

When $a$ is greater than zero the reference position $y_{i}^{\prime}$ compacts the grid nodes on the right and expands the nodes on the left. When $a$ is negative the same overlapping effect occurs on the left side of the grid. Comparing second order to third order, the third order gives a slightly more pronounced overlap effect. Since this method was intended for a narrow range of rotation, using any higher order to increase rotation is not practical.

\subsubsection{X-Y Rotation}

The method of rotation has no special properties to test, the ability to rotate an entire block of grid nodes only requires the same pivot in the encoder and decoder. The amount of rotation depends upon the encoder's ability to find the estimate angle $\theta$. 


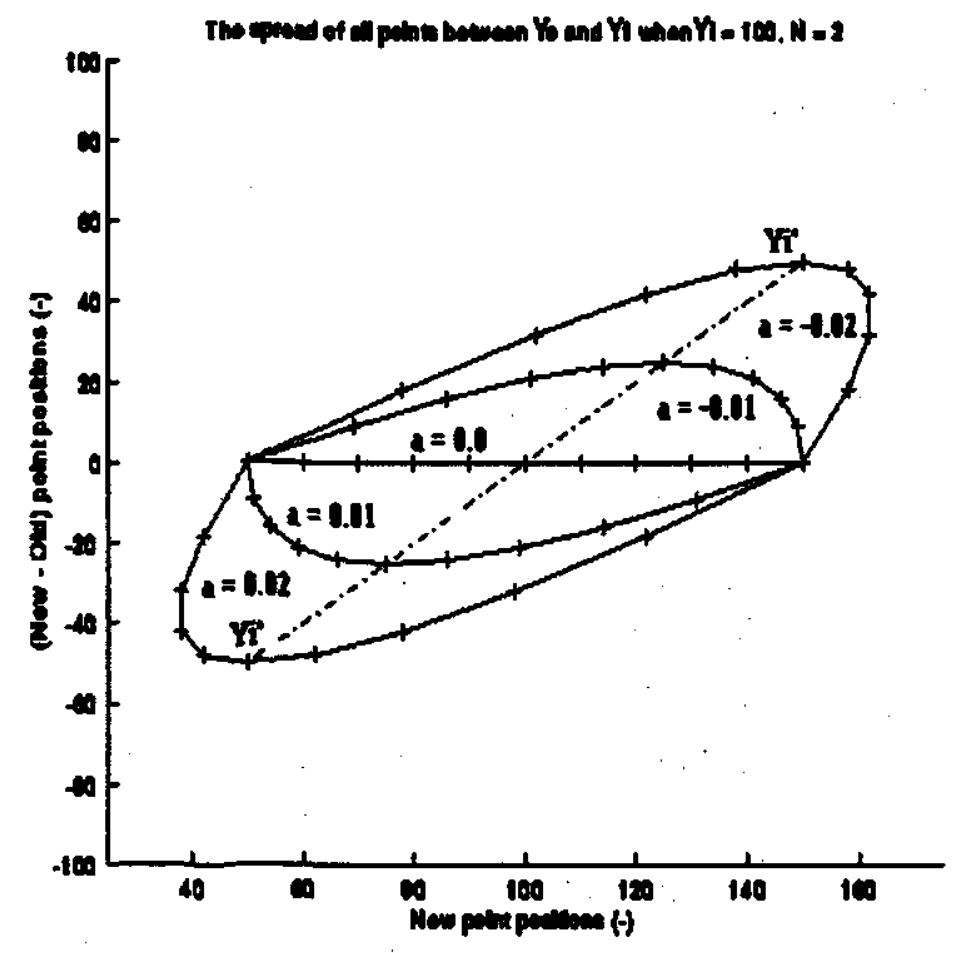

(a)

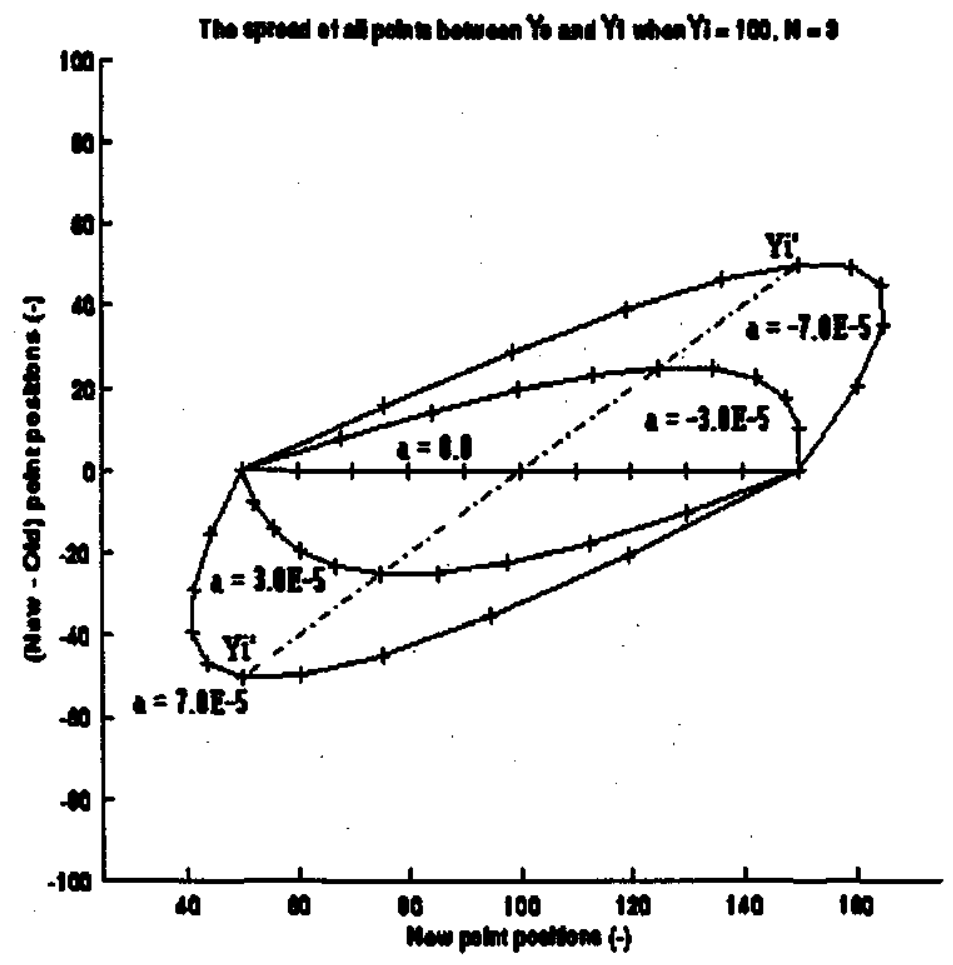

(b)

Figure 6.10 Nonlinear Motion of the Mesh 


\subsection{Image Warping Performance}

Justification of the use of the mesh algorithms can only be rationalized by showing the effect of mesh manipulation on a real image. In the section on warping, bilinear interpolation is favored for its ability to do warping quickly, therefore all examples in this section use bilinear interpolation.

The simulation of eyes and mouth will be looked at first with the use of Attractor/Repellor algorithms.

\subsubsection{The Eyes}

The eye motion is mostly vertical motion, therefore the use of $\beta$ is not necessary. To test the eyes for movement, $n$ will be set to 1.5, 2.0, and 2.5, and $\alpha$ is fixed to 1.10. These parameters are applied to the grid in iterations using the previous slide grid as a source. The eyes in the reference image are partially open, but the desired result is to make the eyes open wider. For each eye, two mass nodes are placed at $(0.01,0.00)$ and $(-0.01,0.00)$ with respect to the center of the eye. In the next three pages of results, each case of $n$ is shown as eight consecutive slides of the eye action, and four examples of grids for every other slide. The right and left labels for the eye grids are from the point of view of Claire's picture. The results show as $n$ increases the amount of movement for the eight frame sequence decreases. 


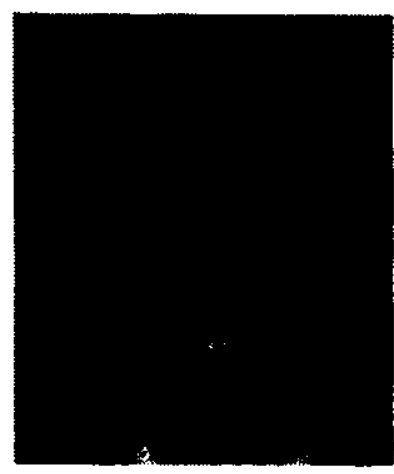

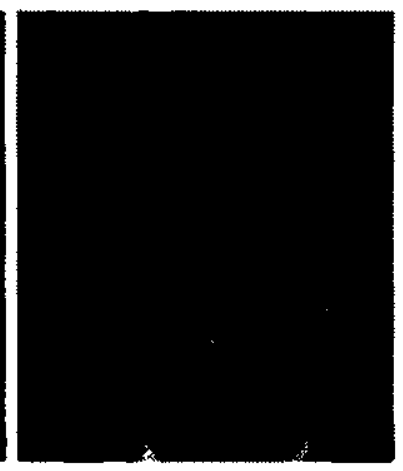

2

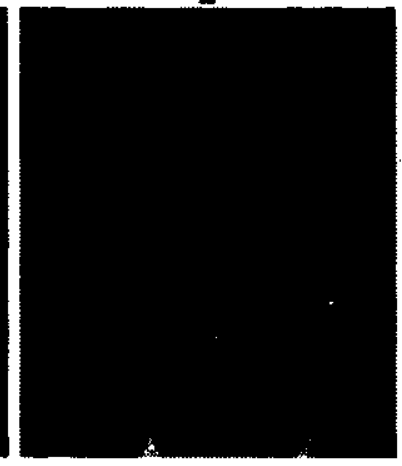

4

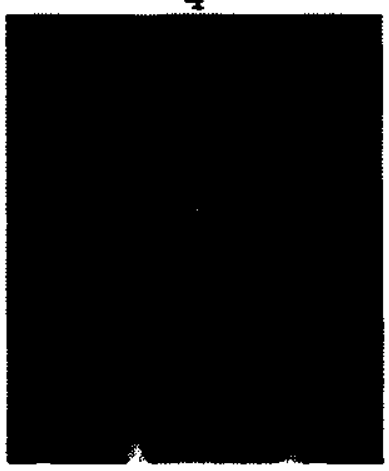

5

6

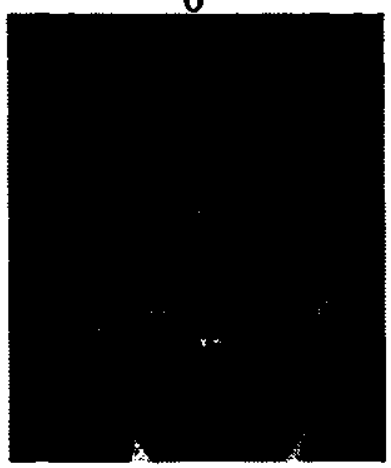

8

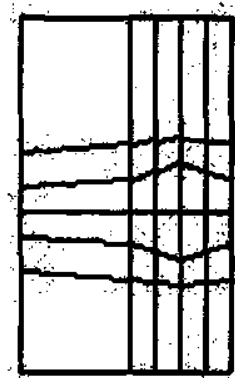

$\mathbf{R}$

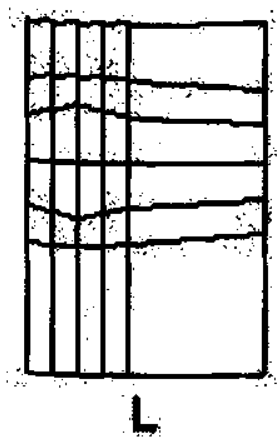

Grid 2
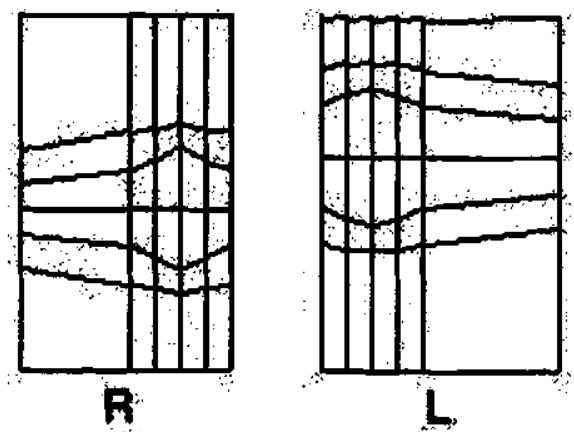

Grid 4

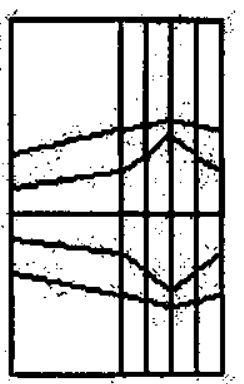

R

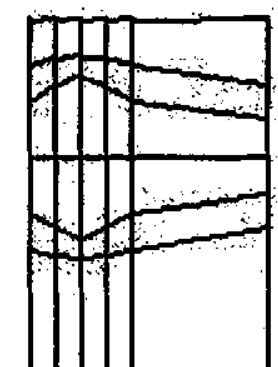

L.

Grid 6

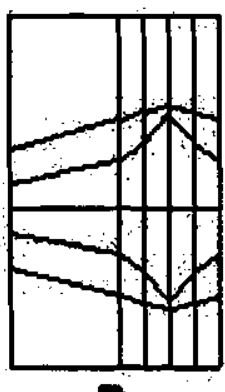

R

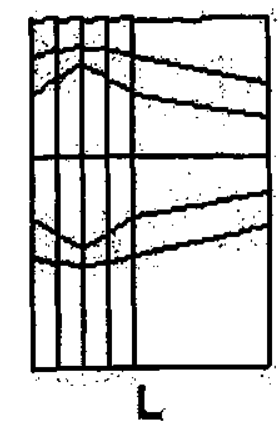

Grid 8

Figure 6.11 Eye Movement for $n=1.5 \alpha=1.10$ 

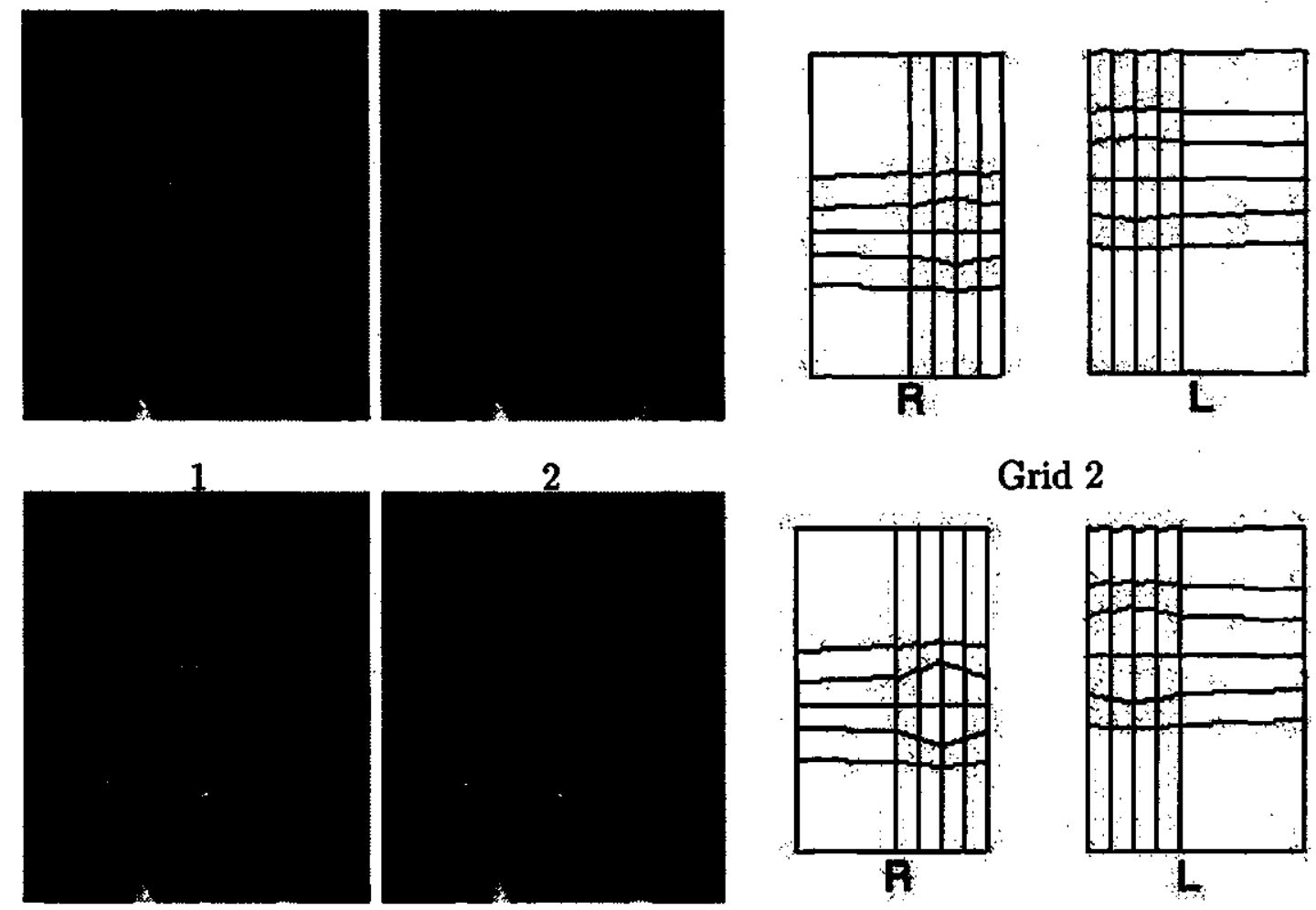

Grid 2
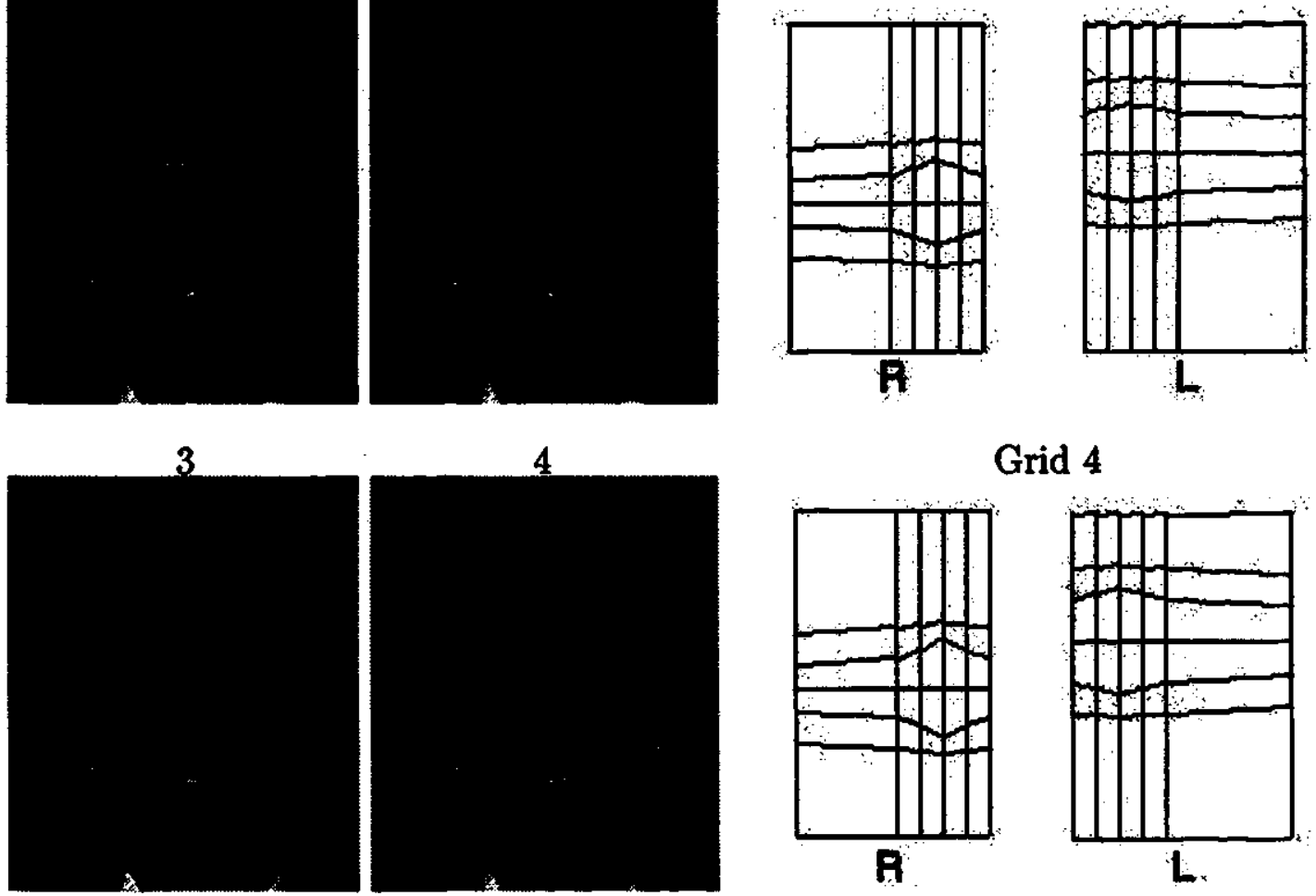

Grid 4

5

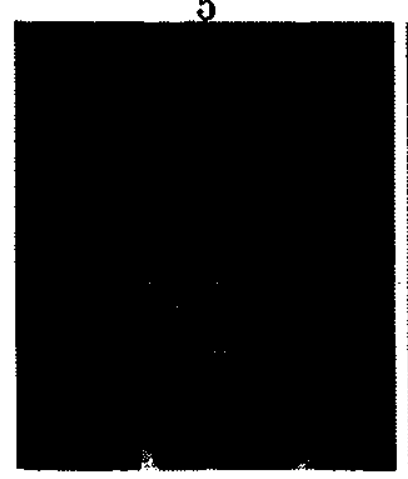

7
6

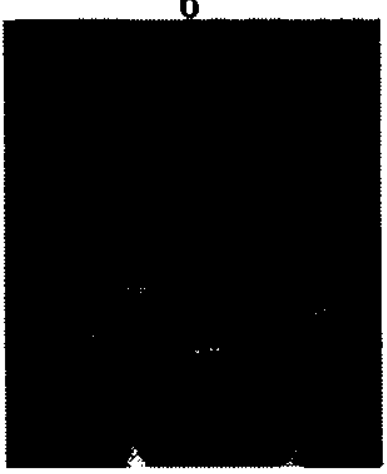

8
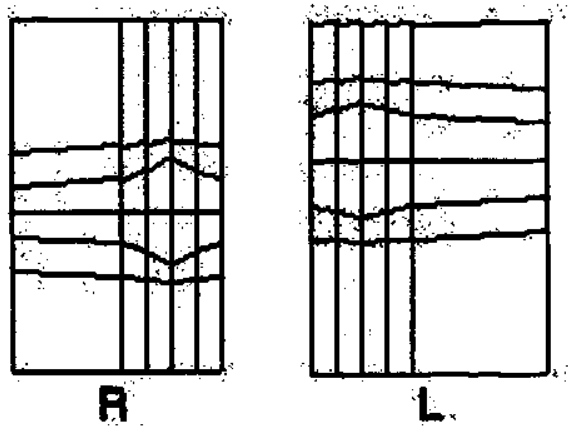

Grid 6

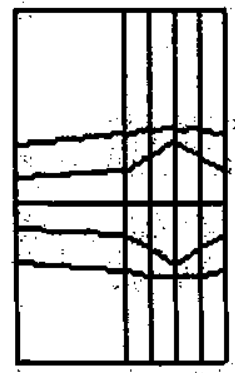

$\mathbf{R}$

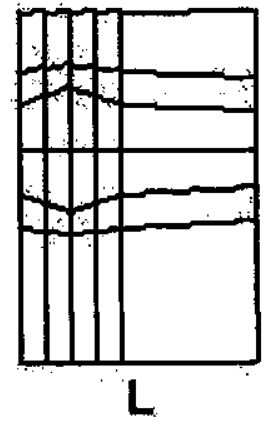

Grid 8

Figure 6.12 Eye Movement for $n=2.0 \alpha=1.10$ 

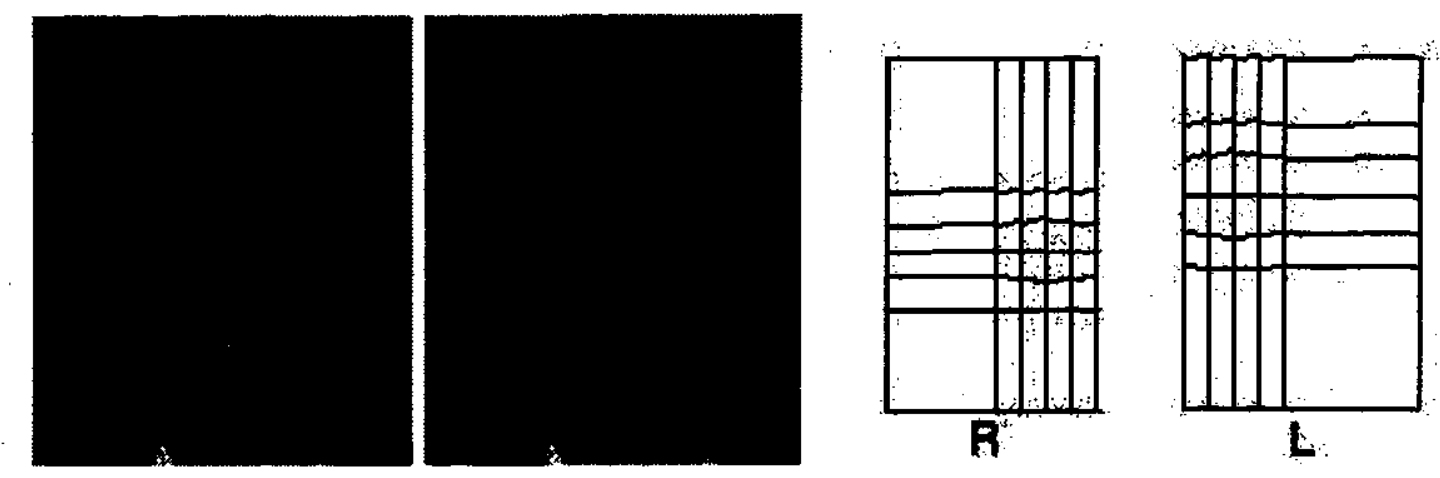

1

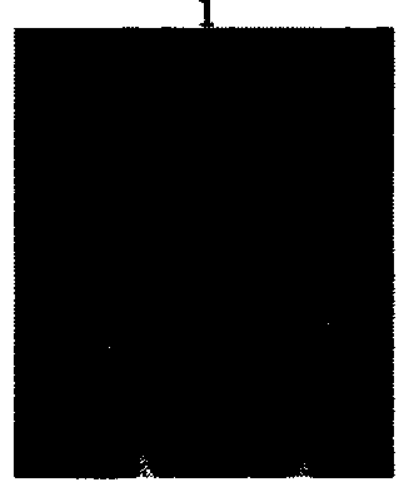

3

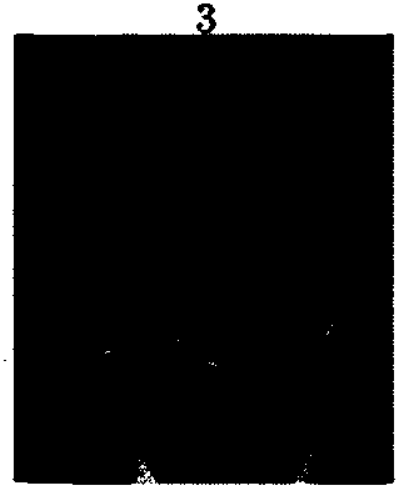

5

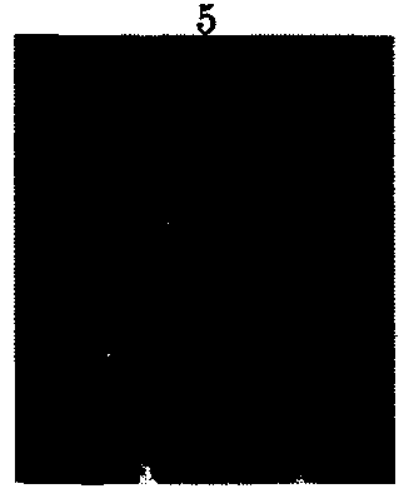

7
2

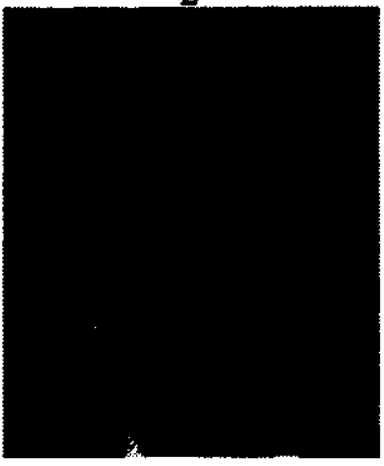

4

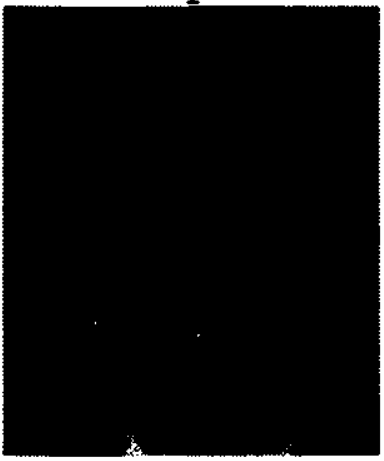

6

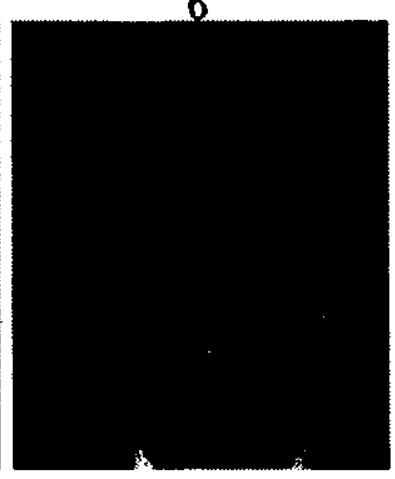

8
Grid 2
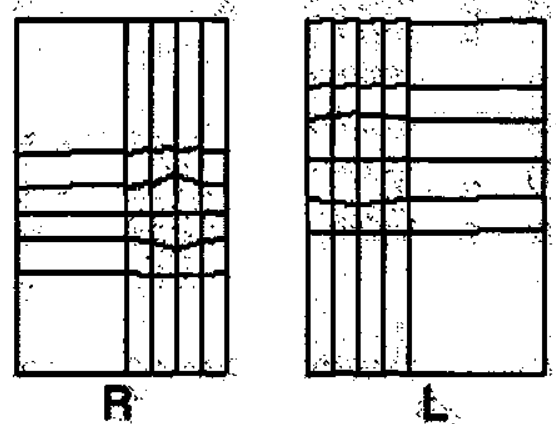

Grid 4
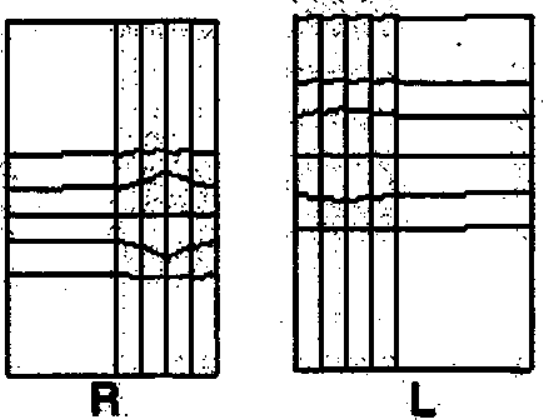

Grid 6
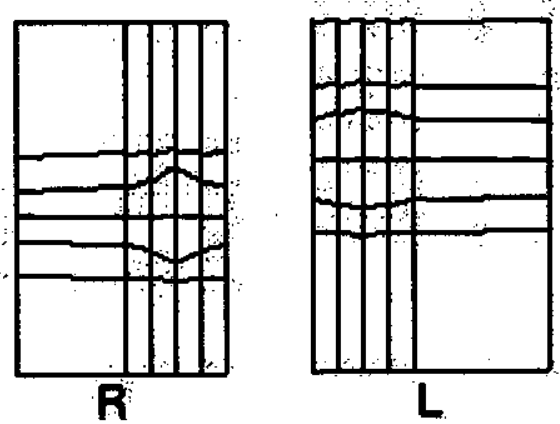

Grid 8

Figure 6.13 Eye Movement for $n=2.5 \alpha=1.10$ 
The choice of $\boldsymbol{n}$ to use depends upon the amount of frame to frame object displacement. From the choices of $n, n=1.5$ gives the greatest frame to frame movement. Yet, the rapid movement has caused bulging to occur in the grid. The use of $n=2.0$ achieves less distortion, but less frame to frame movement. To decide the best $n, \alpha$ is tested.

With the same testing procedure, $\alpha$ is set to $1.05,1.075,1.10$, and 1.15 for a system in which $n$ equals 1.5. The corresponding slides are Fig. 6.14, Fig. 6.15, Fig. 6.11, and Fig. 6.16. The expected result is less movement for a smaller $\alpha$, but the choice of $\alpha$ must not allow an unnatural appearance in the sequence.

When the eye is tested with $\alpha$ at 1.05 and 1.075 , the results look similar to the previous tests of $n=2.5$ and $n=2.0$ in Fig. 6.13 and Fig. 6.12. When $\alpha$ is greater than 1.10 , such as 1.15 , the distortion becomes unavoidable. The result of these tests indicate movement can be accomplished with $\alpha$ or $n$, but $\alpha$ is a more flexible parameter. 

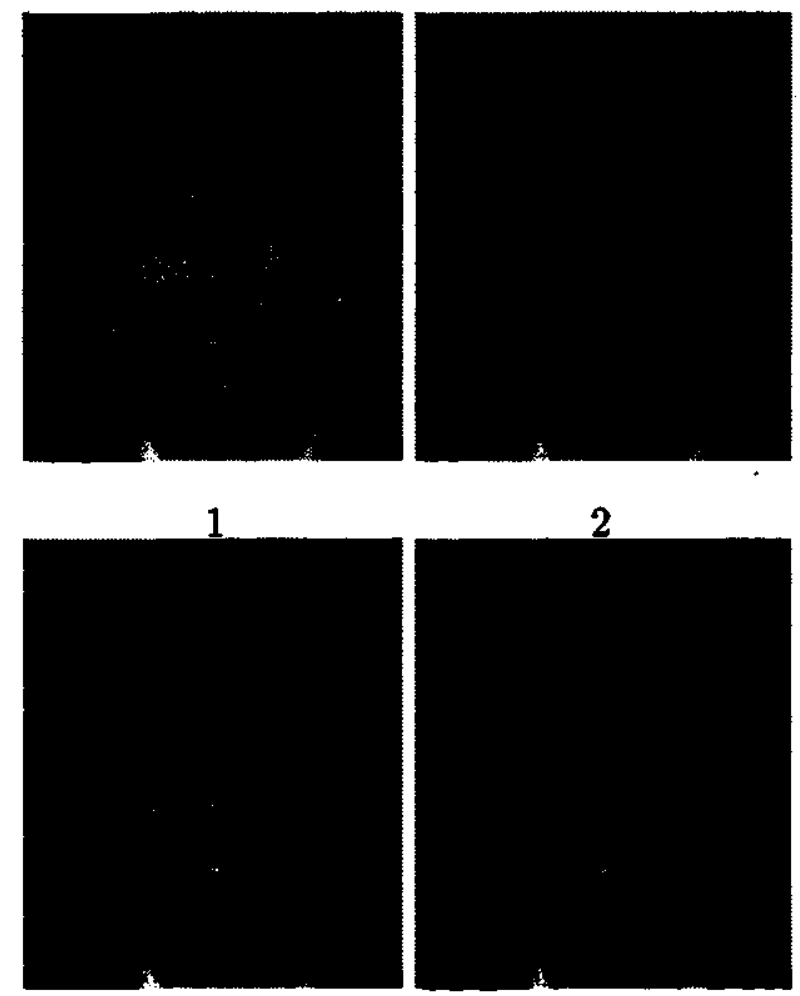

2
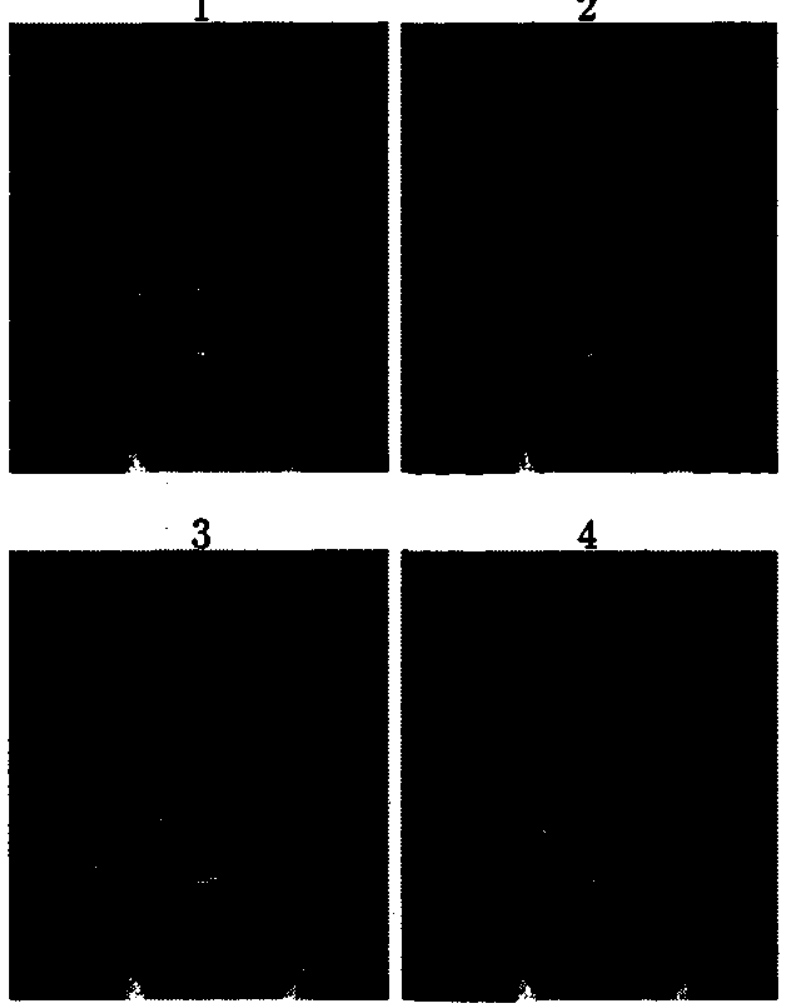

5

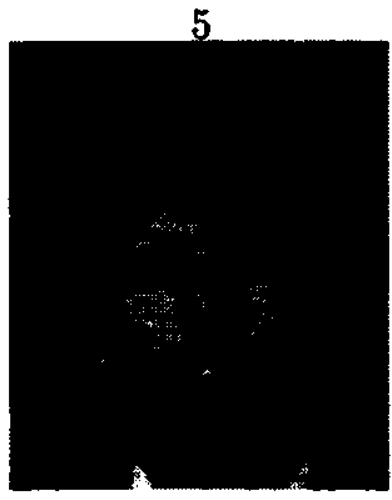

7

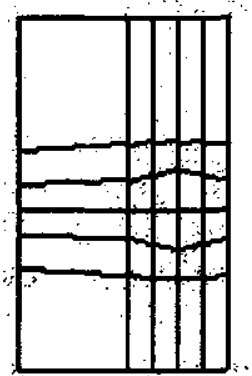

$\mathbf{R}$

Grid 2

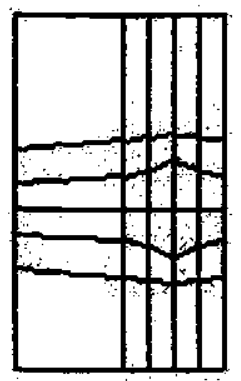

R

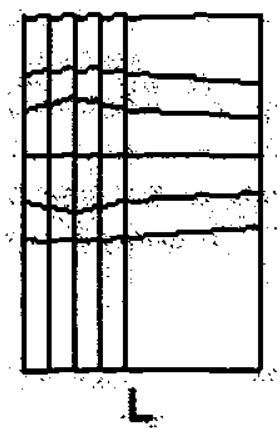

Grid 4

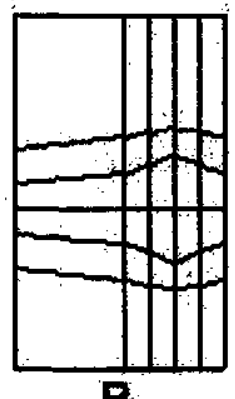

$\mathbf{R}$

Grid 6
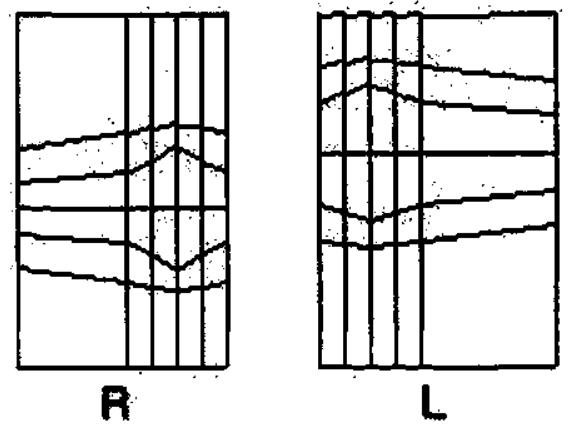

Grid 8

Figure 6.14 Eye Movement for $n=1.5 \alpha=1.05$ 


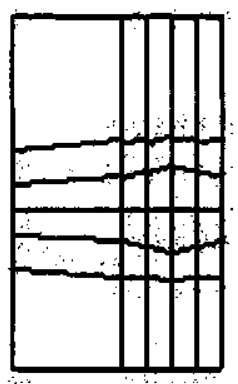

n

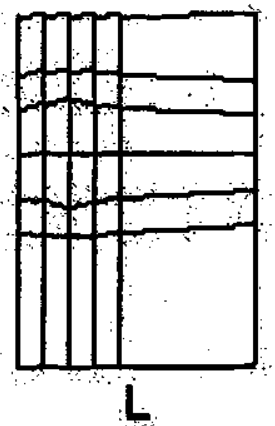

Grid 2

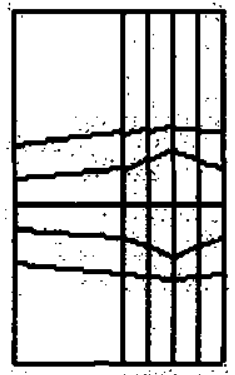

R

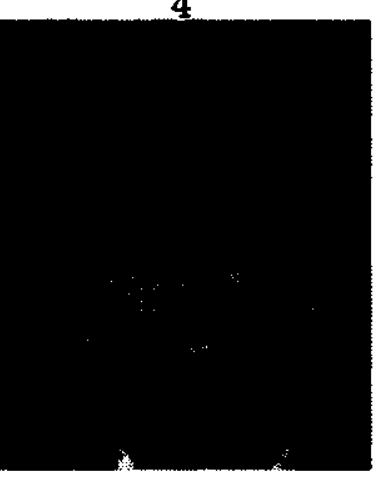

6

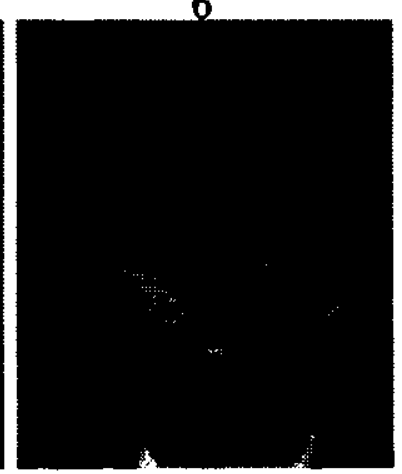

8

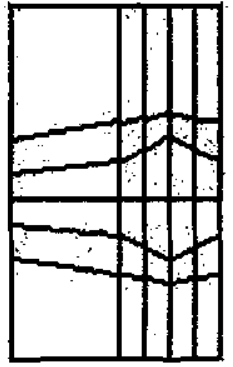

R

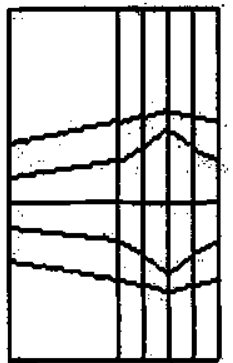

R

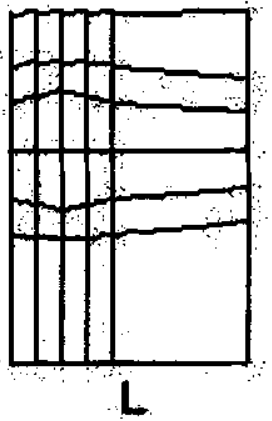

Grid 4

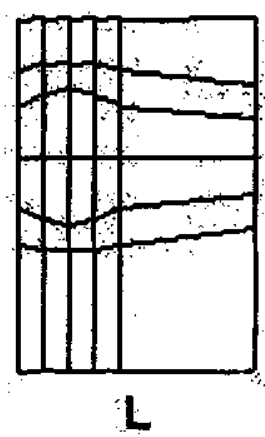

Grid 6

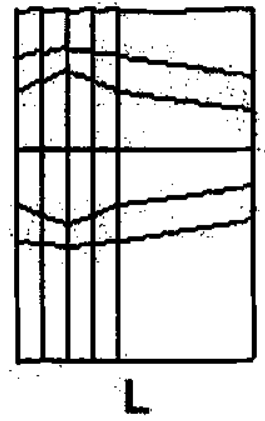

Grid 8

Figure 6.15 Eye Movement for $n=1.5 \alpha=1.075$ 

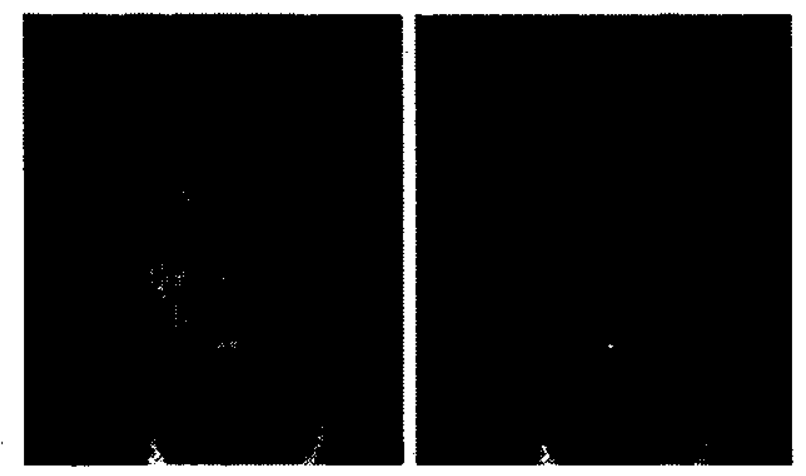

2
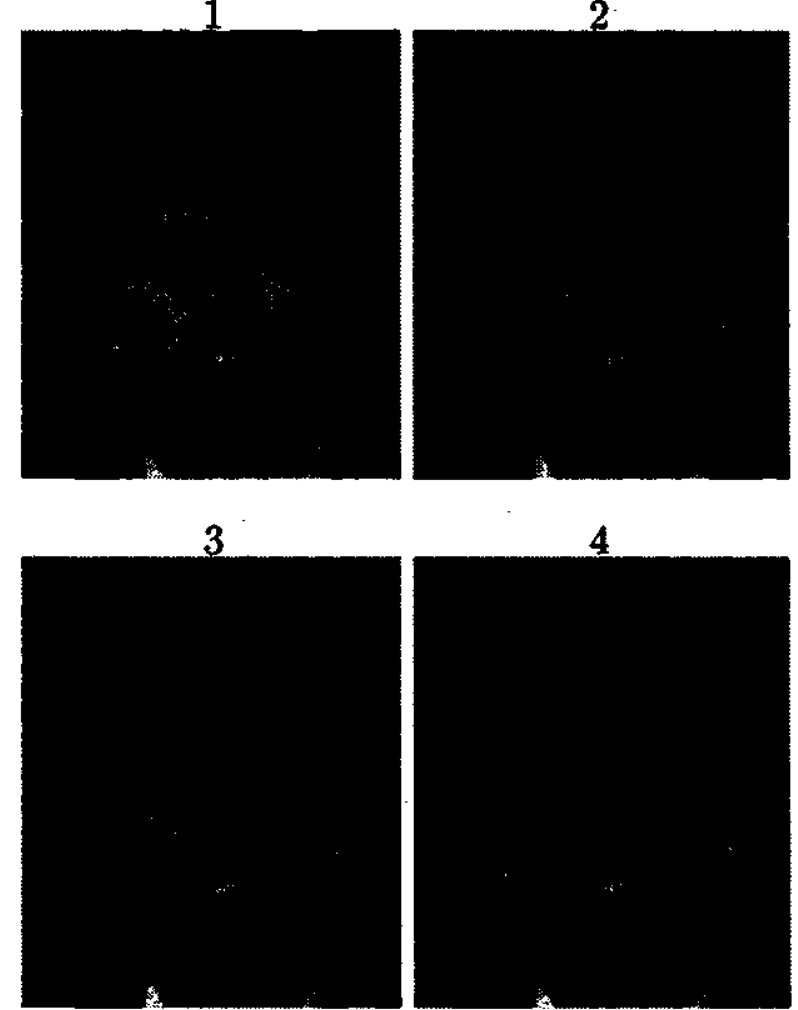

5

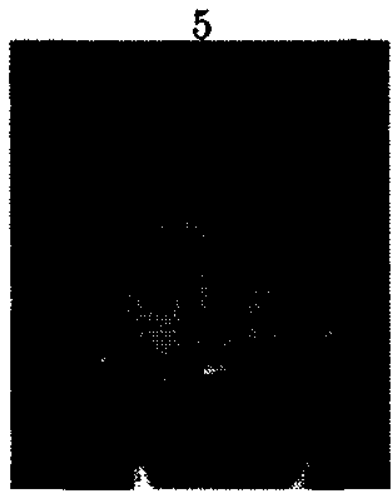

7
4

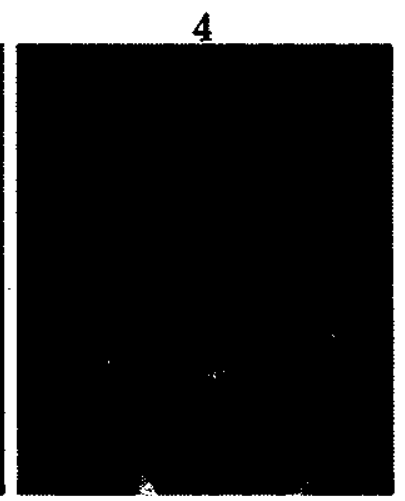

6

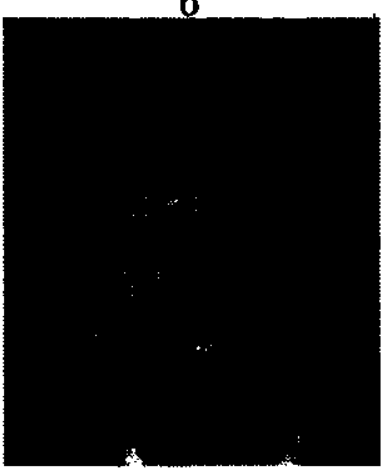

8

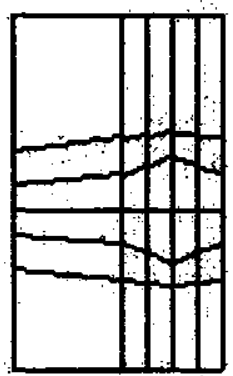

R

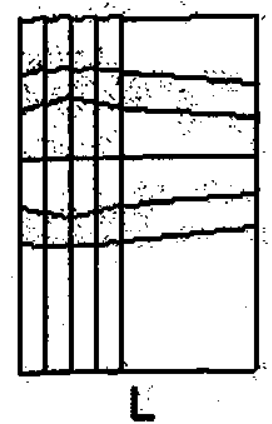

Grid 2
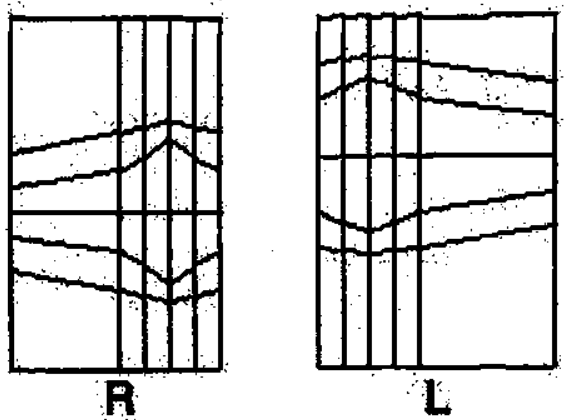

Grid 4
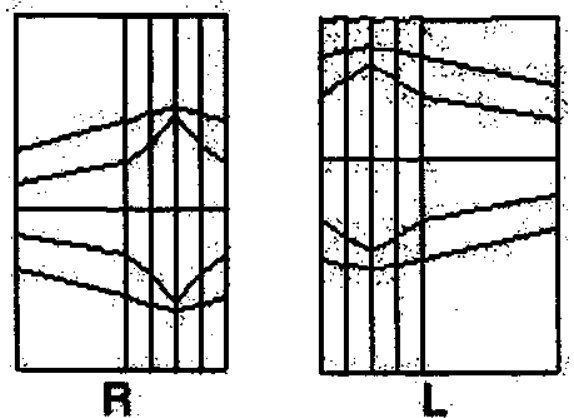

Grid 6
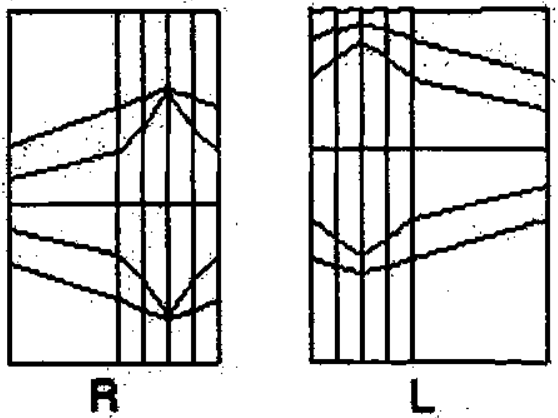

Grid 8

Figure 6.16 Eye Movement for $n=1.5 \alpha=1.15$ 
The last two sequences of slides, Fig. 6.17 and Fig. 6.18, show a sequence for opening and closing of the eyes to display the range of motion possible. The grids for the closing of the eyes show the eye grids collapsing. To avoid image distortion a limit for the range of the attractor motion must be placed to prevent the inner most lines from crossing over. 

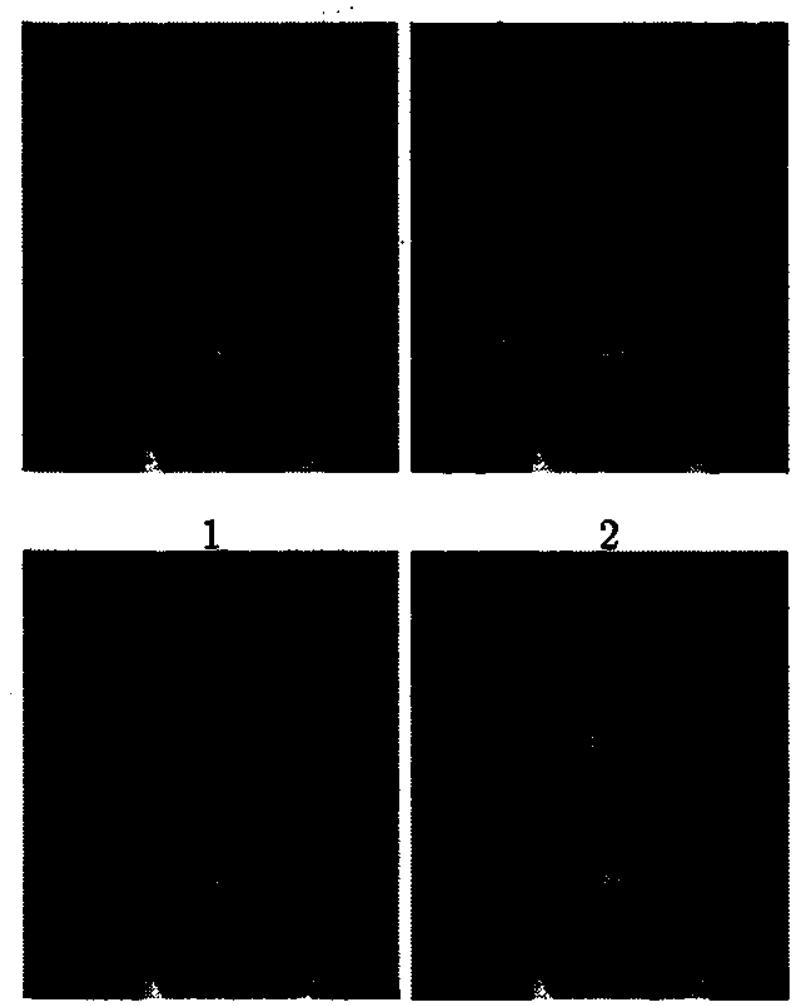

2

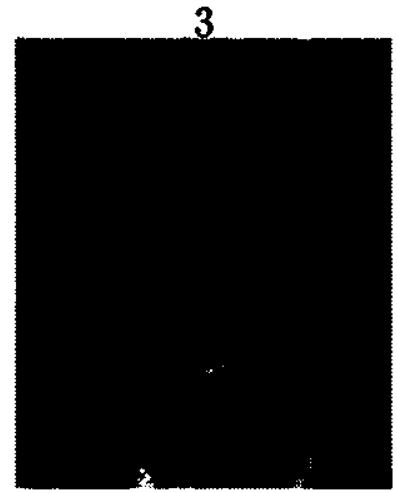

4
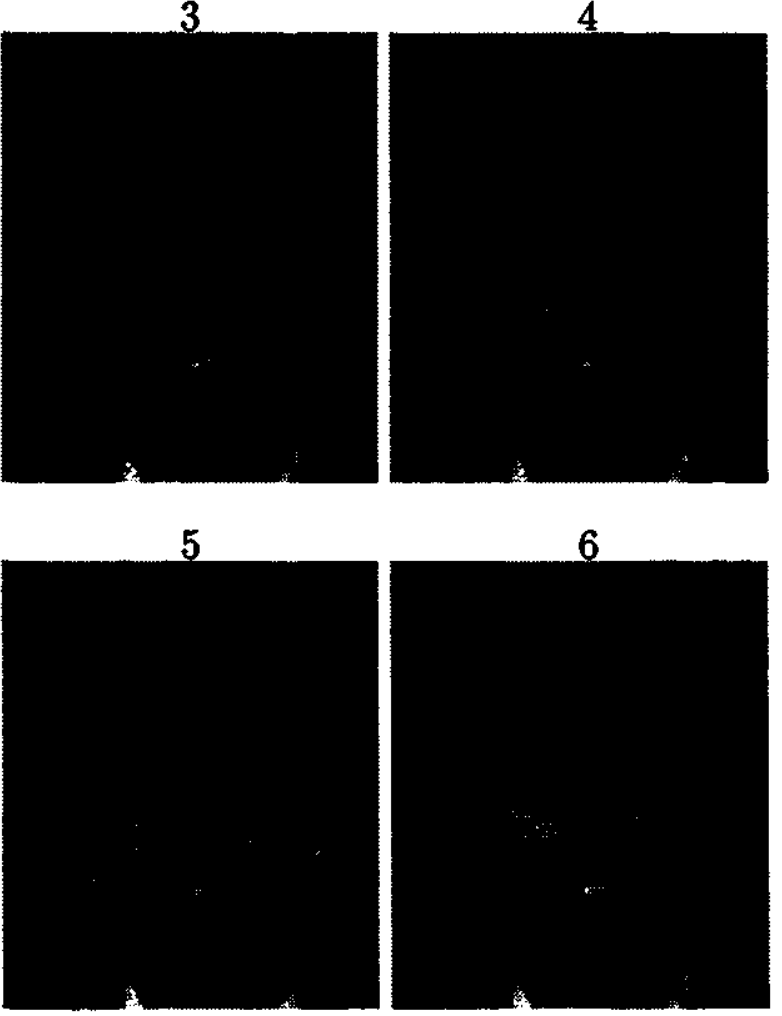

7

8

Figure 6.17 Typical Eye Opening Action 

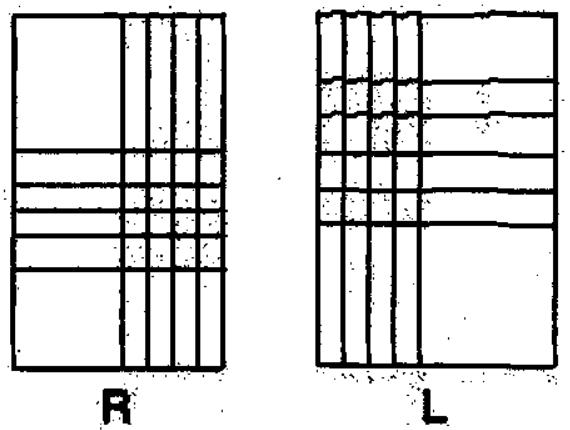

Grid 2
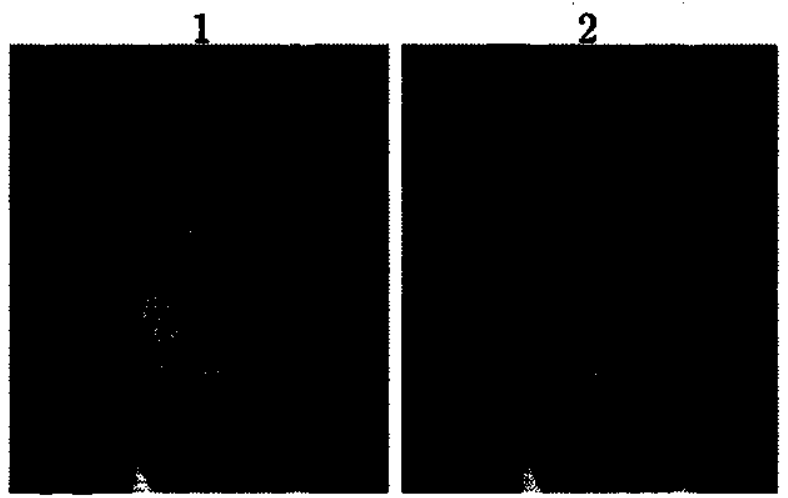

3
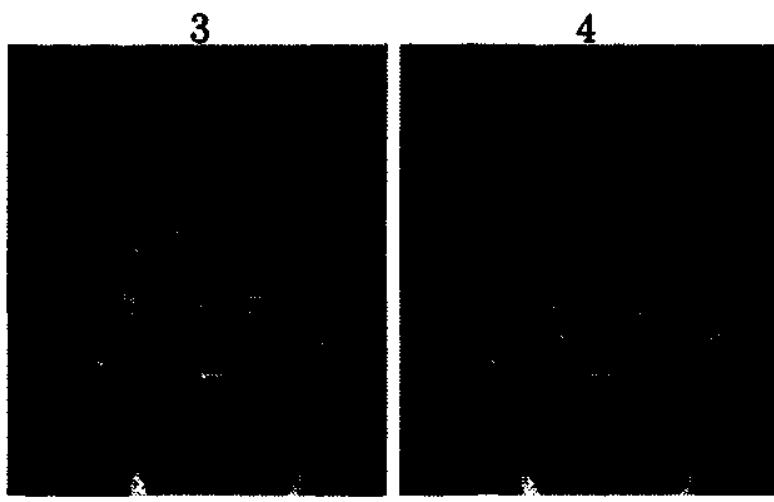

5

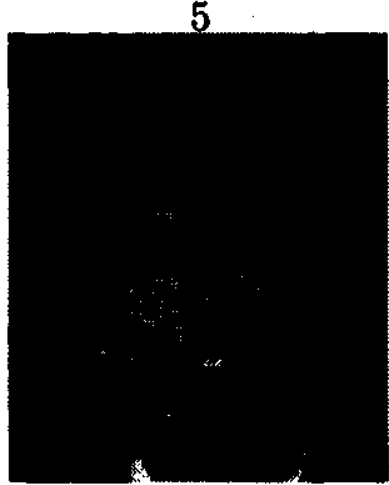

7
6

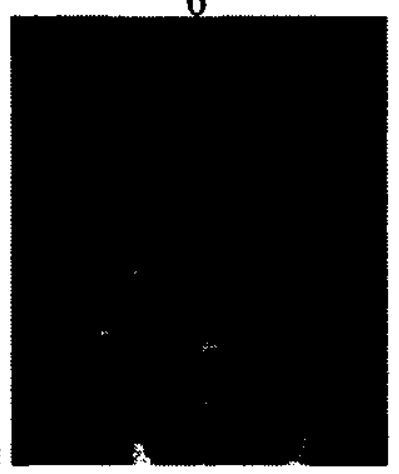

8

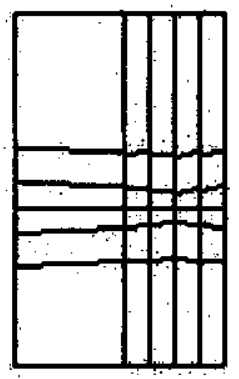

R

Grid 4

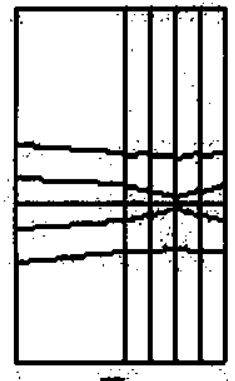

R

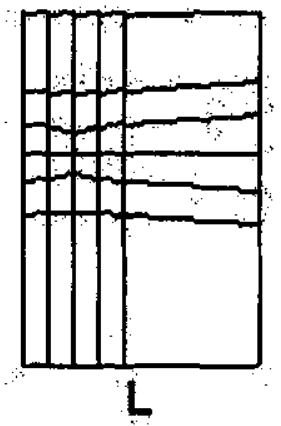

Grid 6
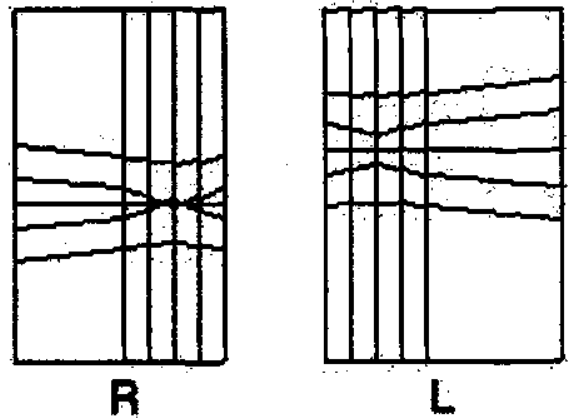

Grid 8

Figure 6.18 Typical Eye Closing Action 


\subsubsection{The Mouth}

The movement of the mouth is more versatile than the eyes. $\alpha$ and $\beta$ are equally important to simulate movement of the mouth in the two directions. For the test grid, four mass nodes are arranged in a square pattern about the center of the mouth. The testing procedure will be the same as the eyes. In the first test, $\alpha$ and $\beta$ are fixed and $n$ is the variable. For the conditions of $n$ equal to $1.5,2.0$, and 2.5 , the action are represented in Fig. 6.19, Fig. 6.20, and Fig. 6.21. The choice of $\beta$ is 0.5 and $\alpha$ is 1.1 .

When $n$ equals 1.5 the mouth is forced open in both directions creating an oval shape. As the mesh is spread apart the grid lines of the mouth become compact leaving a large open area in the middle. If the inner polygons that make up the mouth become too large compared to the reference grid's polygons the algorithm can not accurately paint the mouth. This is because there are too few pixels from the reference rectangles to fill the enlarged polygon. For $n=2.0$ and 2.5 , the mouth does not open as wide and therefore no distortion occurs. Using the larger values of $n$ would require a larger choice of $\alpha$ to achieve the same movement. Doing this will achieve less distortion since the distribution of grid lines is more even, but the range of $\alpha$ is limited. 

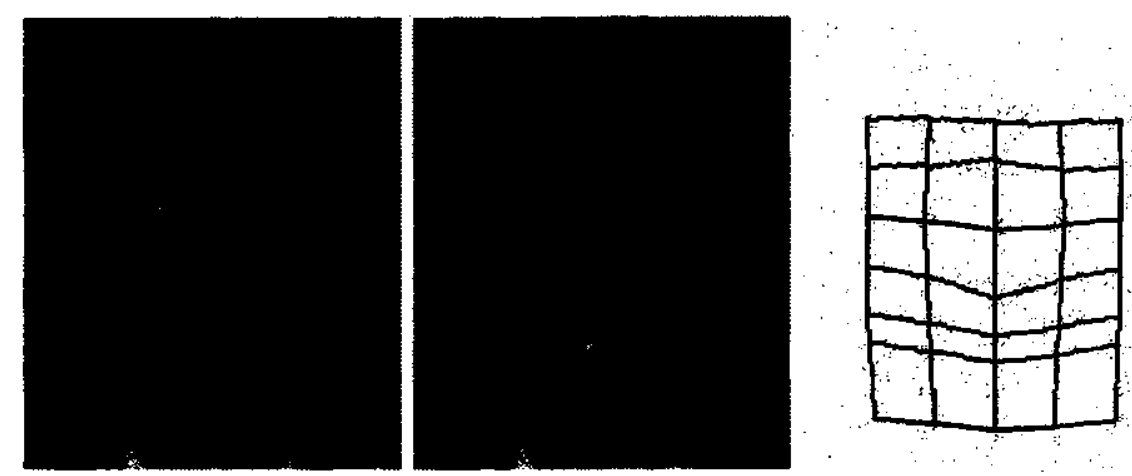

1

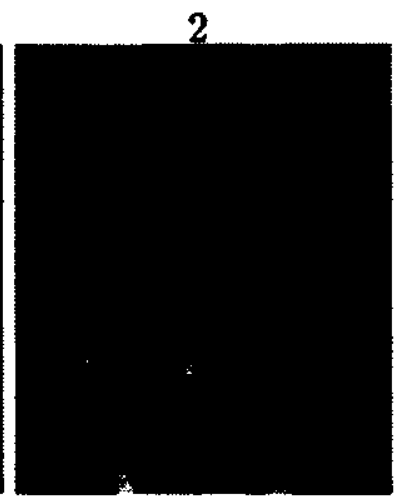

Grid 2

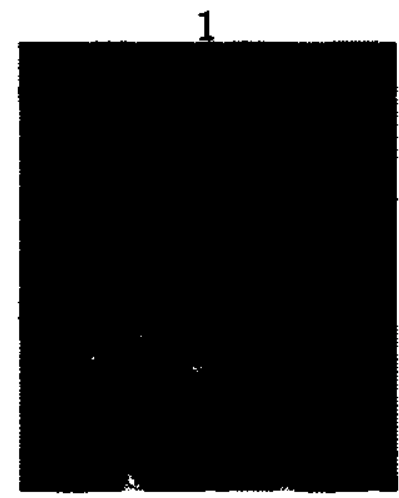

3

4

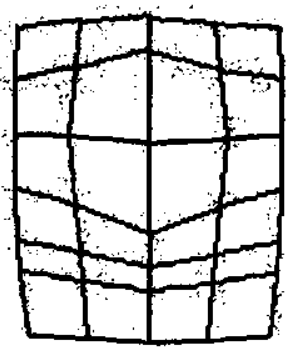

Grid 4
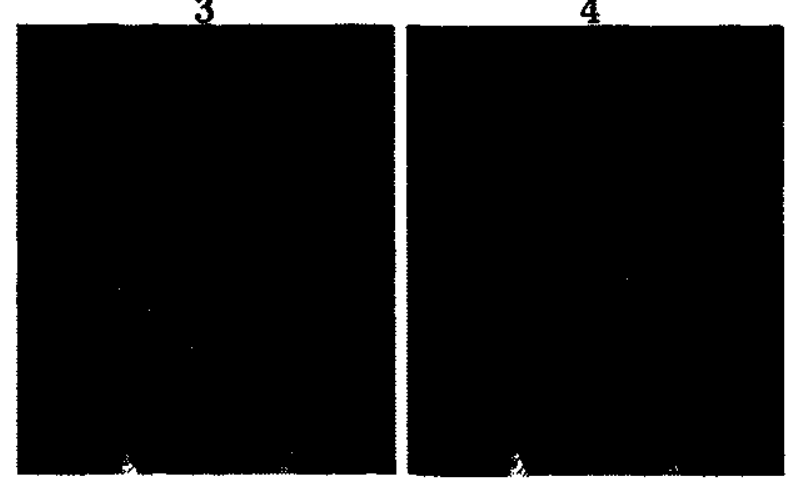

5

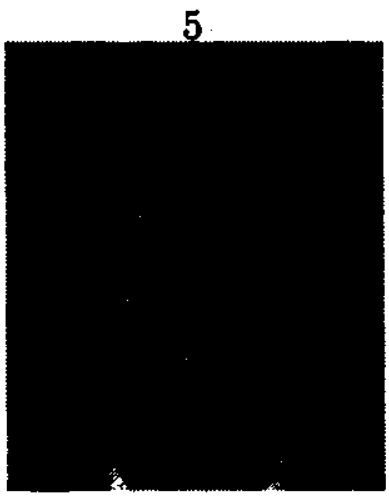

6

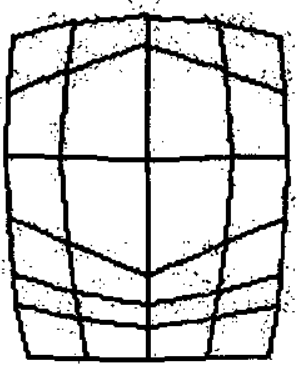

7

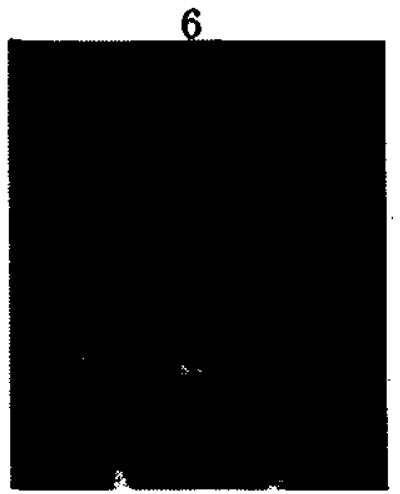

8

Grid 6

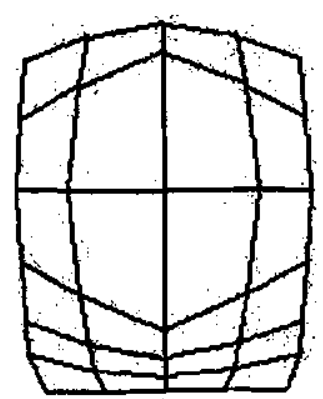

Grid 8

Figure 6.19 Mouth Movement for $n=1.5 \alpha=1.10$ and $\beta=$ 0.50 

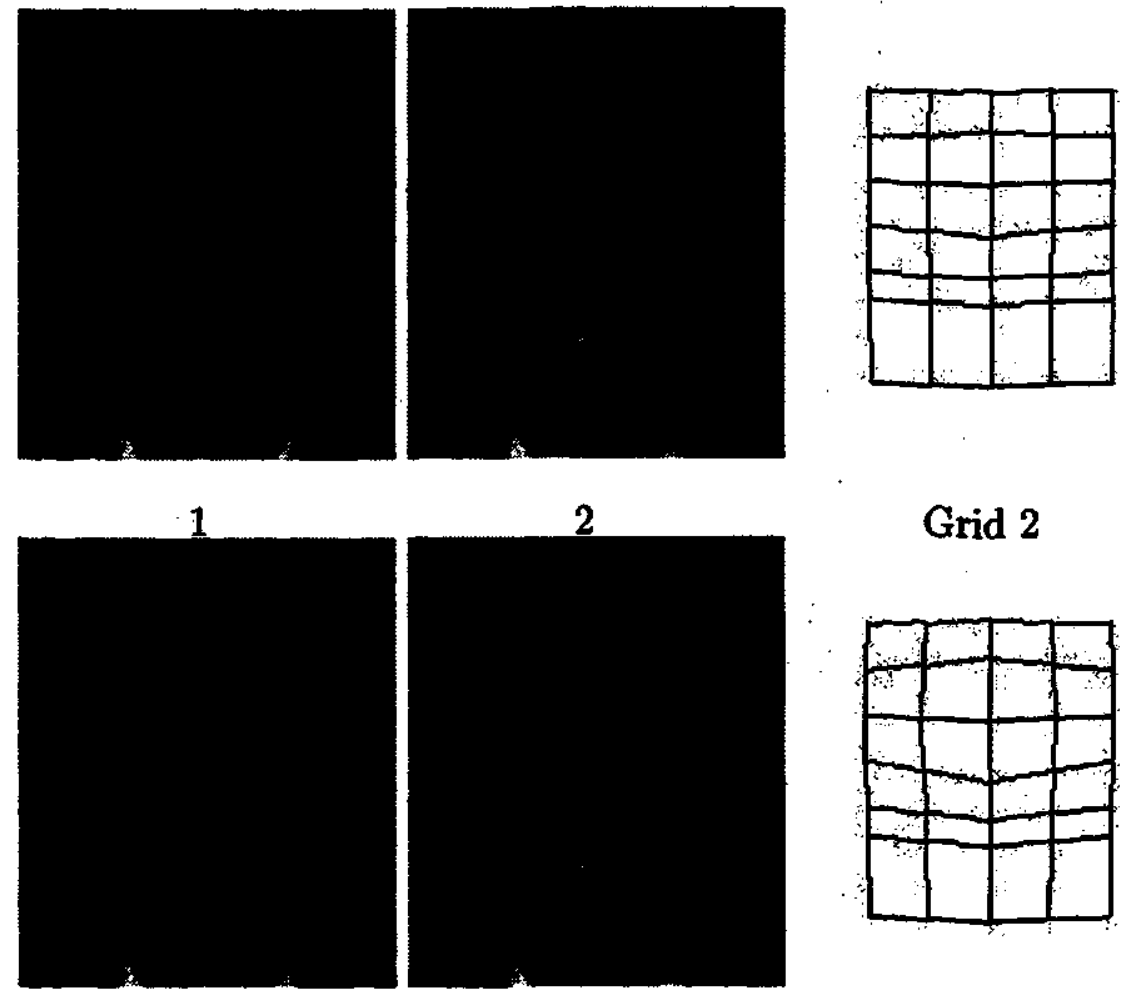

Grid 2

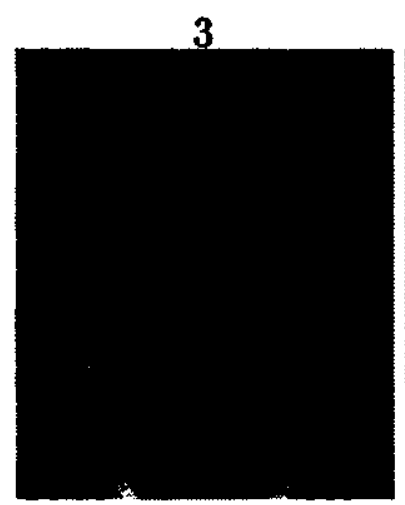

4

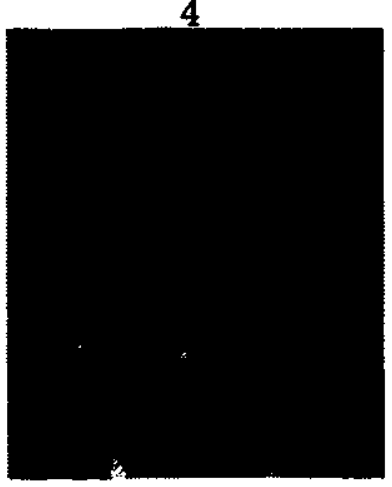

5

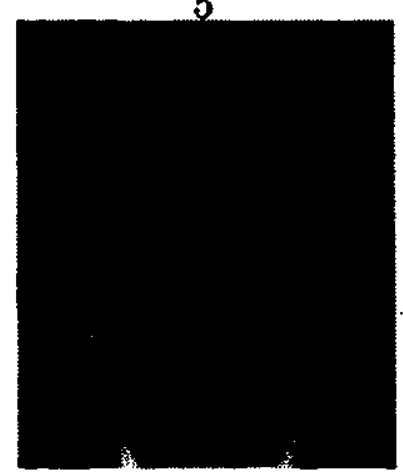

7
6

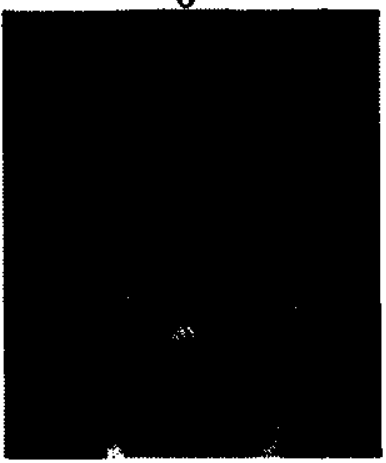

8
Grid 4
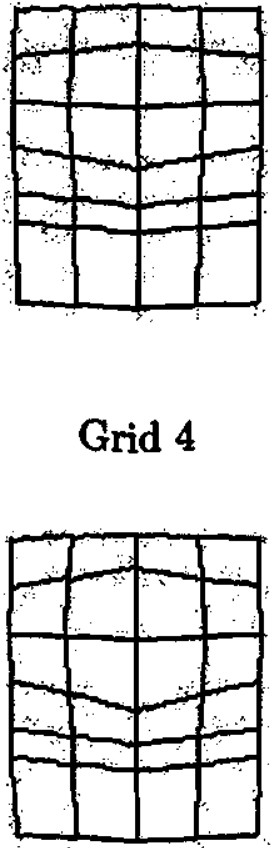

Grid 6

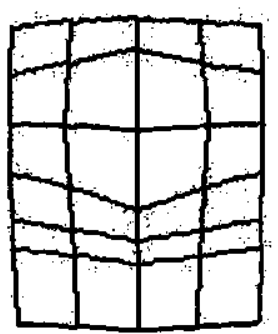

Grid 8

Figure 6.20 Mouth Movement for $n=2 \alpha=1.10$ and $\beta=$ 0.50 

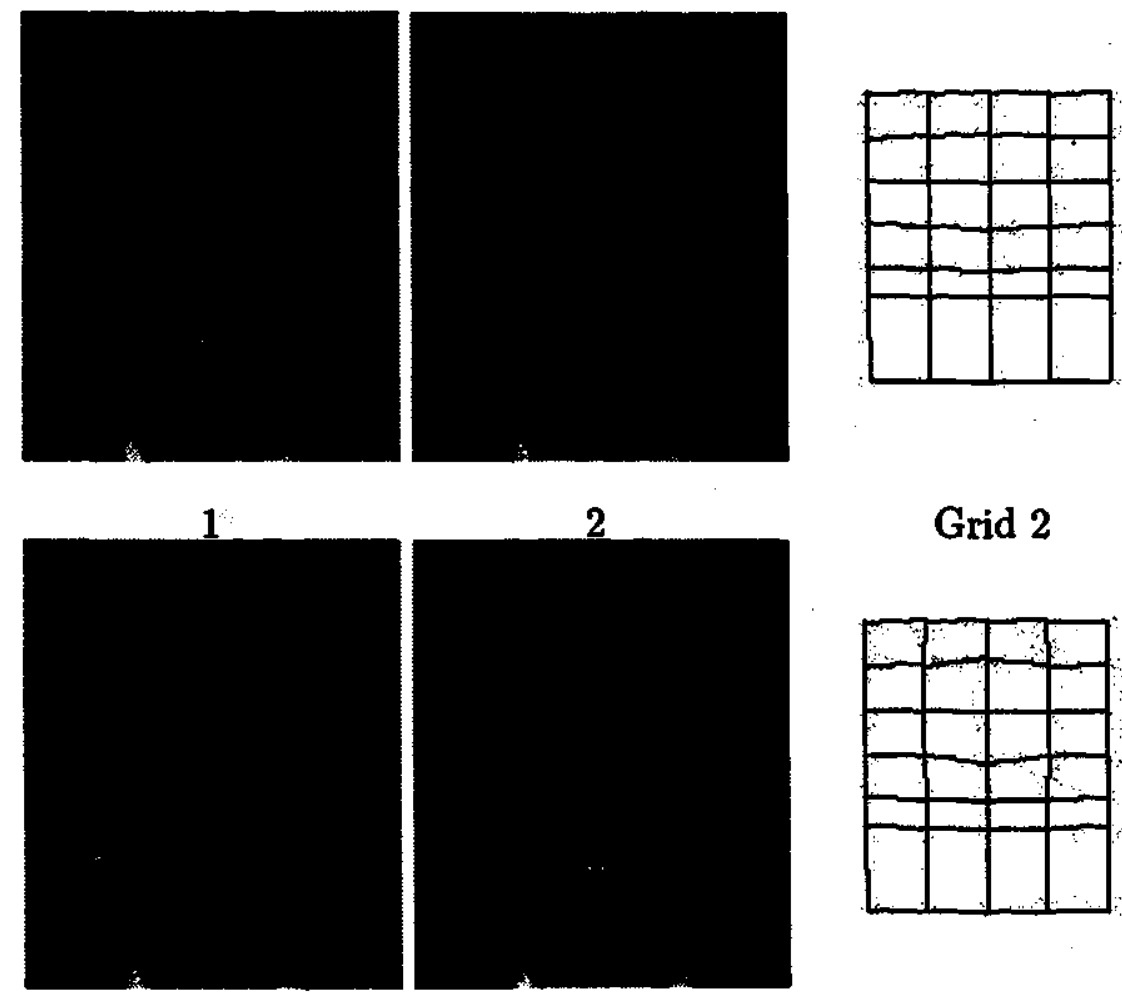

Grid 2
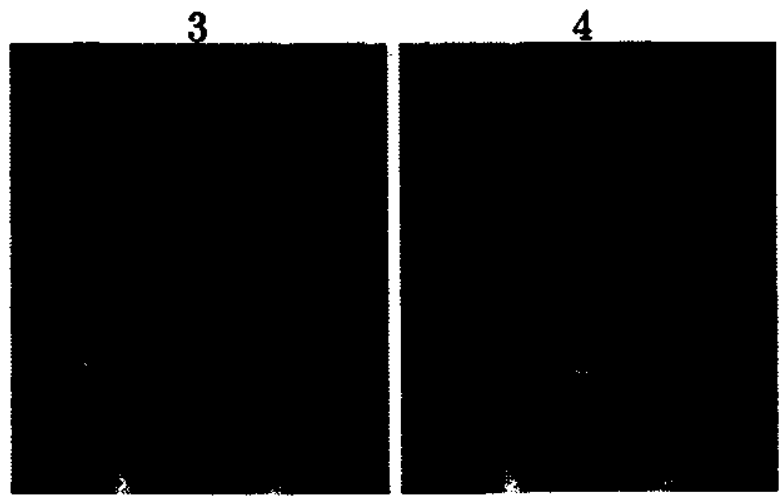

Grid 4

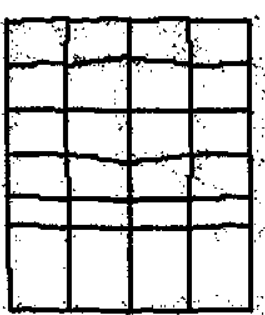

5

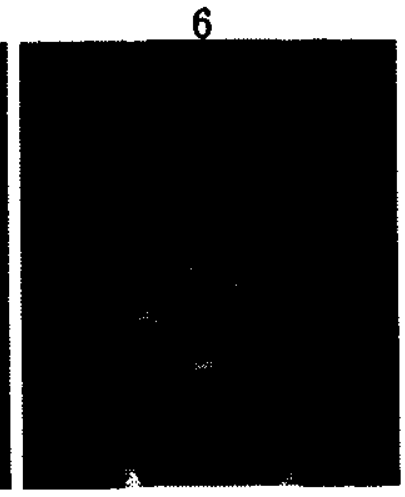

Grid 6

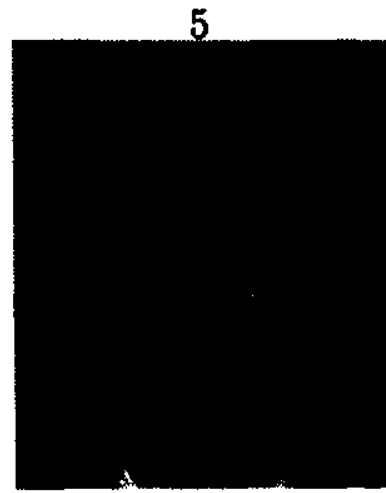

7

8
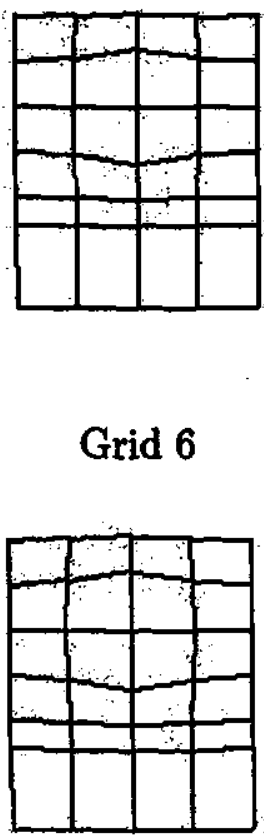

Grid 8

Figure 6.21 Mouth Movement for $n=2.5 \alpha=1.10$ and $\beta=$ 0.50 
In the second mouth $A / R$ test, $\alpha$ and $\beta$ are the variables and $n$ is set to 1.5. The purpose of the test is to show what results can occur by increasing $\alpha$ and $\beta$. In the three figures, Fig. 6.22,Fig. 6.23, and Fig. $6.24 \alpha$ is increased from 1.013, to 1.025, to 1.05 and $\beta$ is increased from 0.25 ,to 0.37 , to 0.50 . Using $\alpha$ and $\beta$ gives two distinct directions of movement. Interesting results of the tests show the choice of $\alpha$ is more sensitive to movement than $\beta$. The use of $\beta$ produces only limited grid movement, but the images show the mouth would look unnatural if $\beta$ was increases further. 

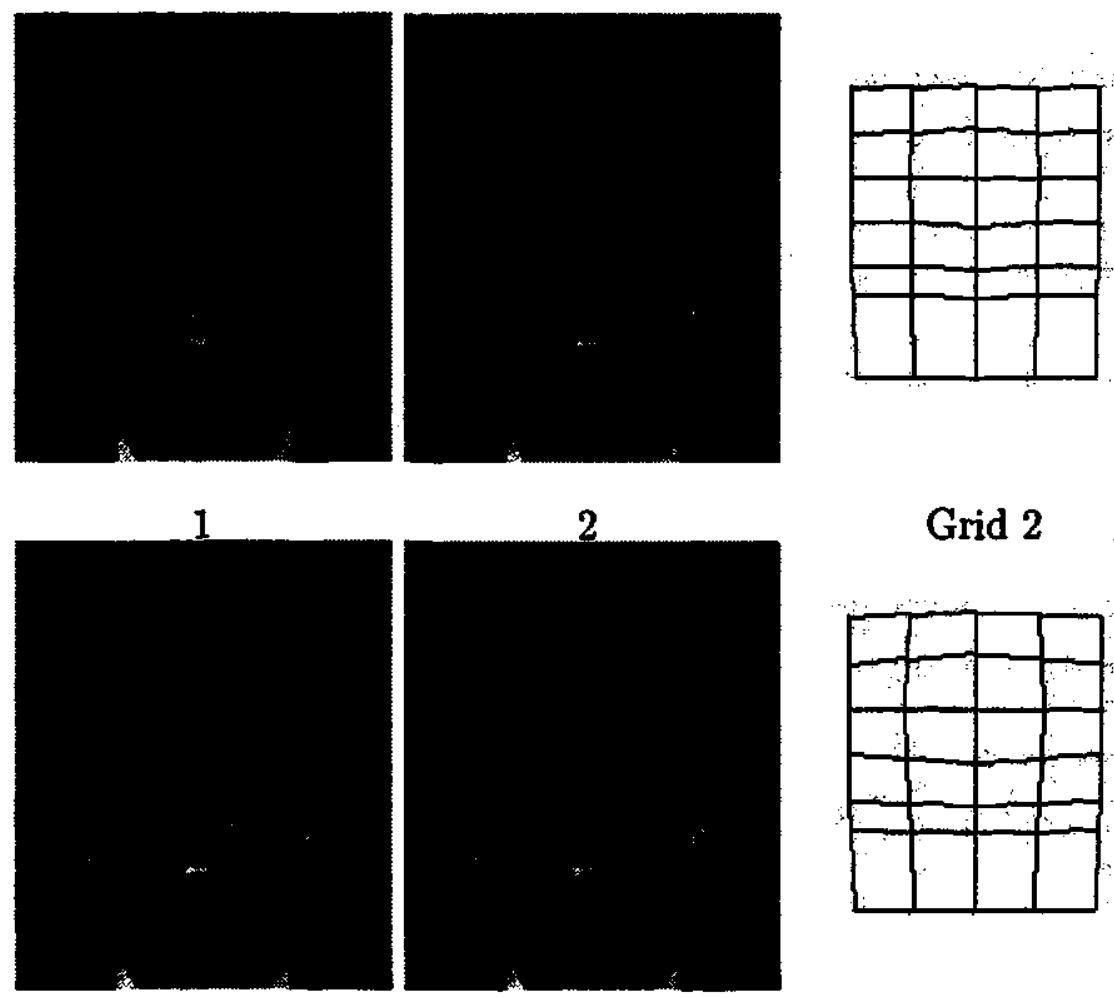

\section{Grid 2}

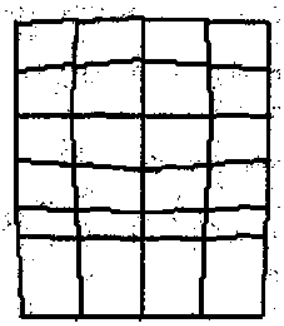

3

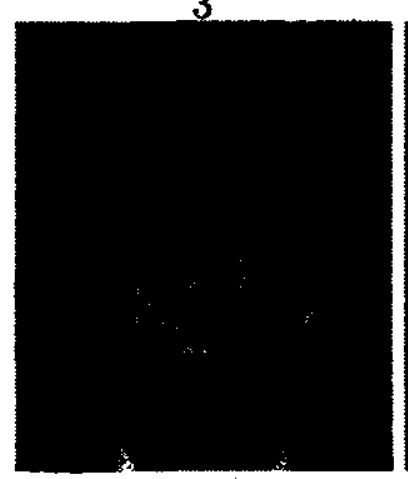

5

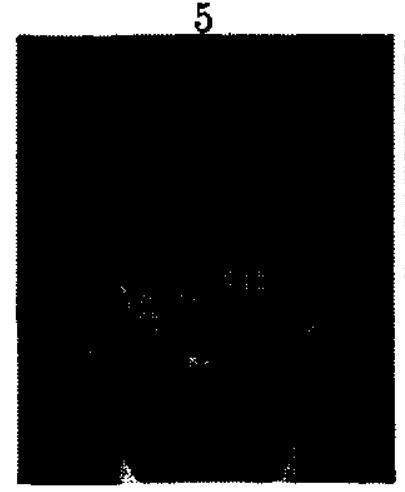

7
4

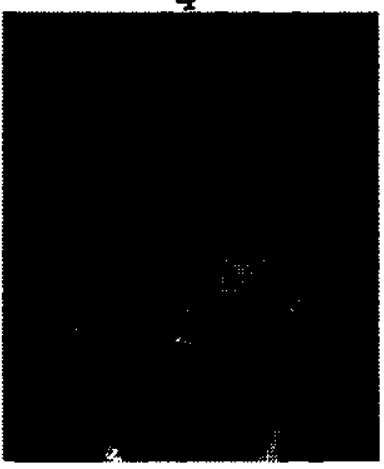

6

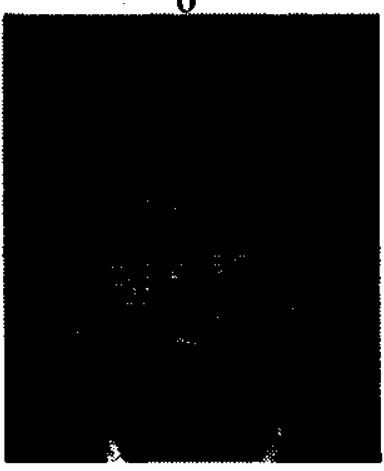

8
Grid 4

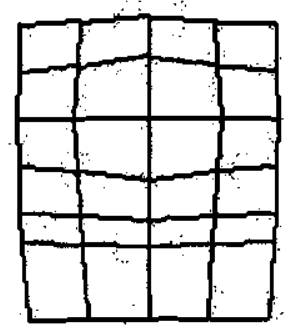

Grid 6

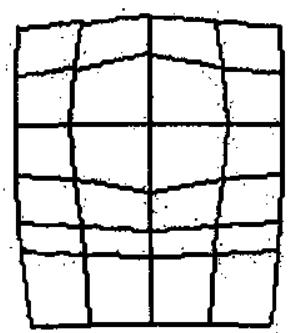

Grid 8

Figure 6.22 Mouth Movement for $n=1.5 \alpha=1.013$ and $\beta$

$$
=0.25
$$



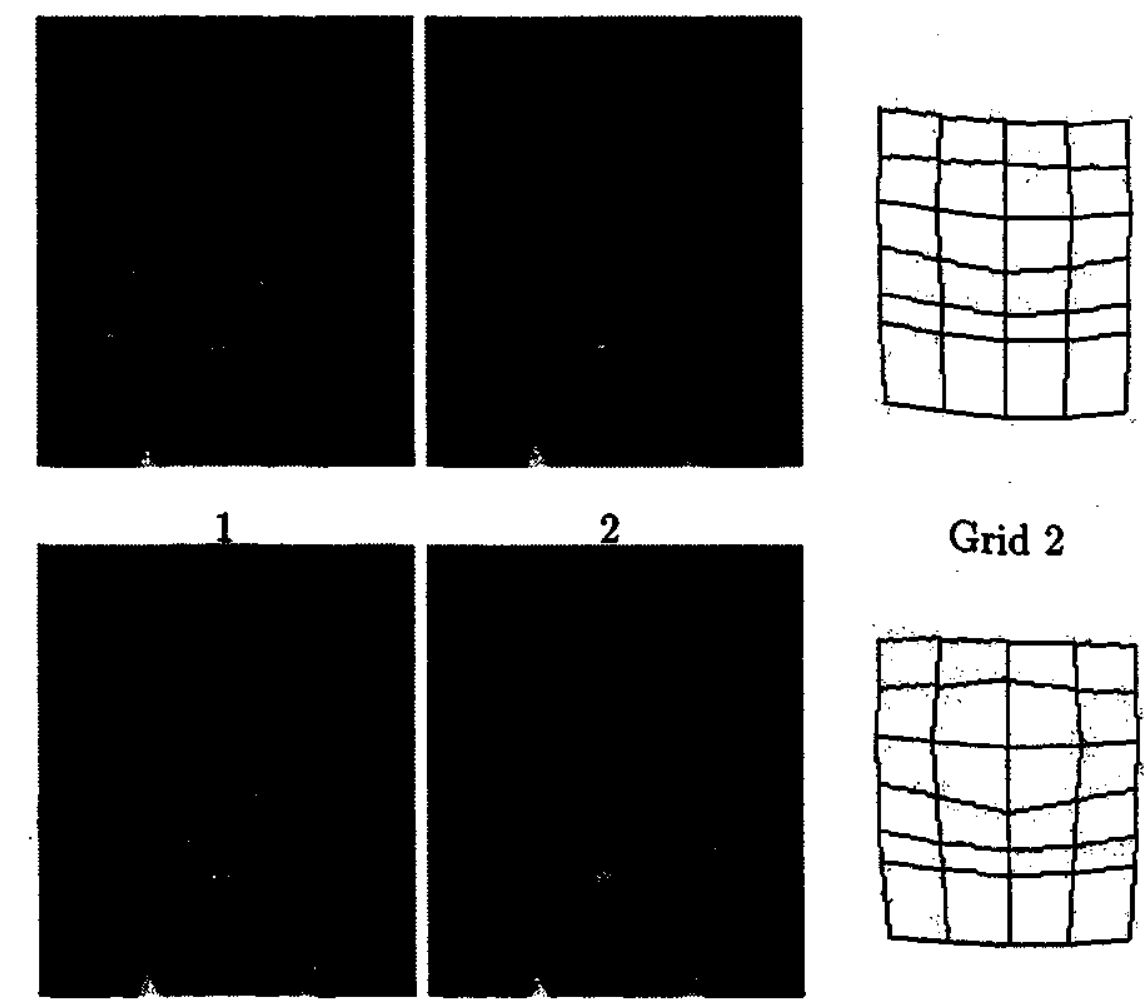

Grid 2
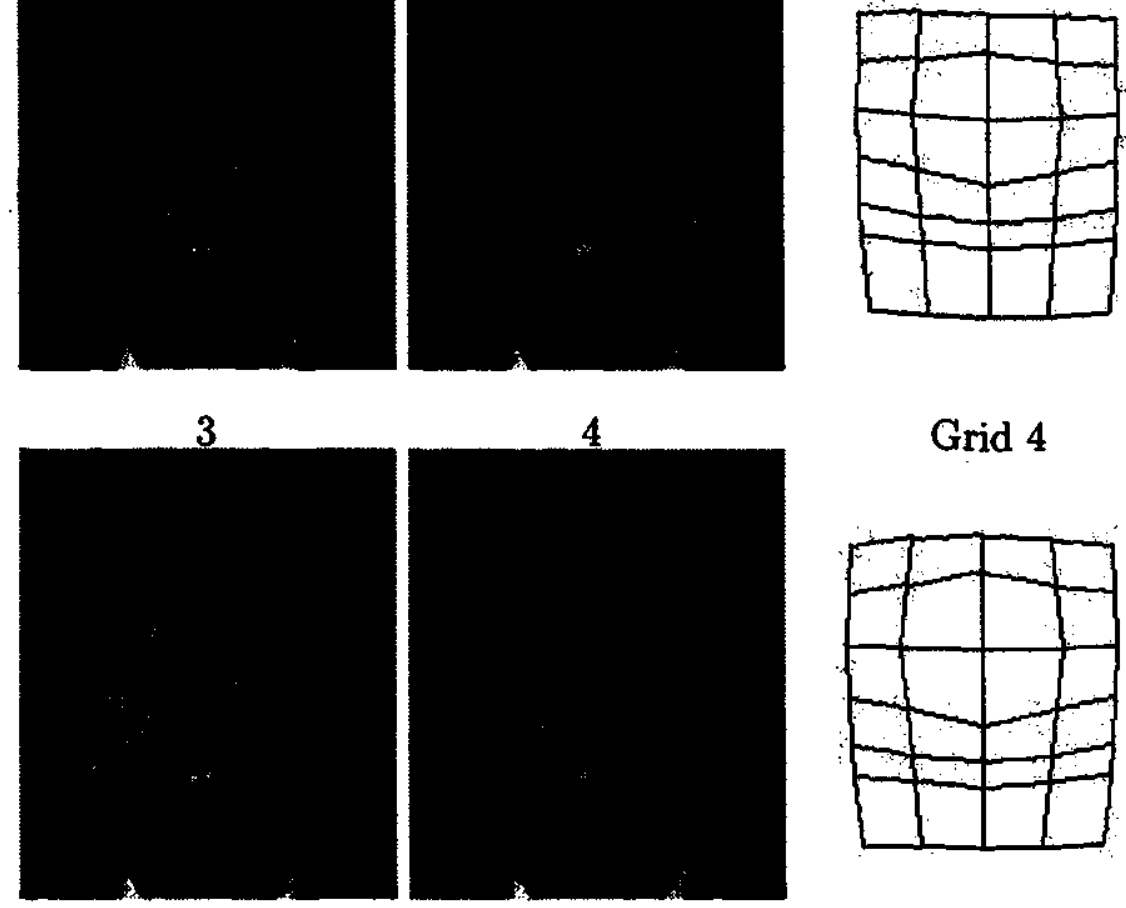

Grid 4

5

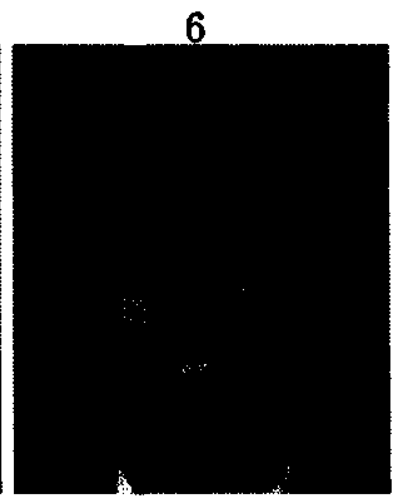

7

8

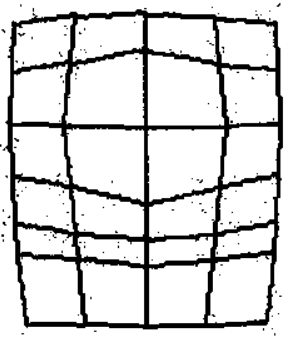

Grid 6
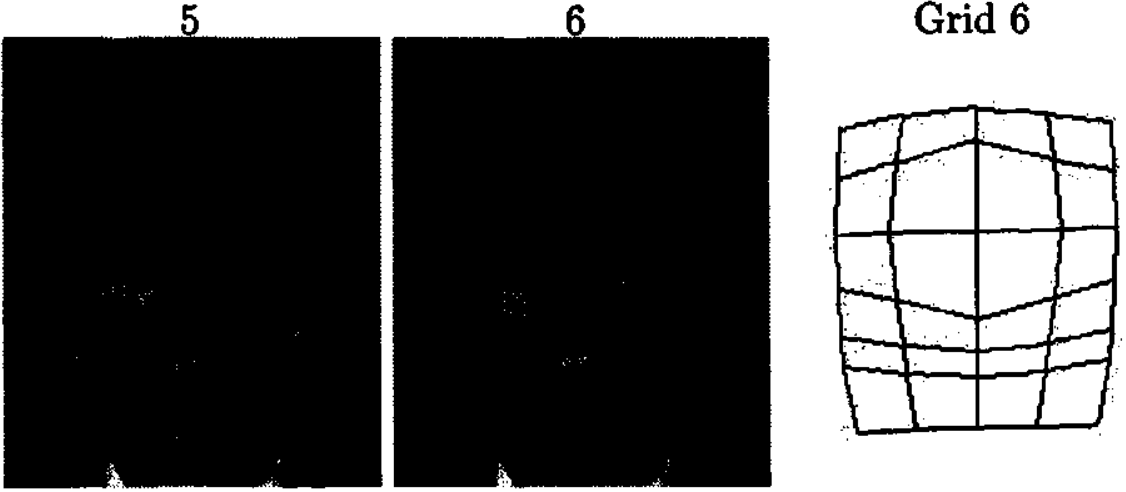

Grid 8

Figure 6.23 Mouth Movement for $n=1.5 \alpha=1.025$ and $\beta$

$$
=0.37
$$



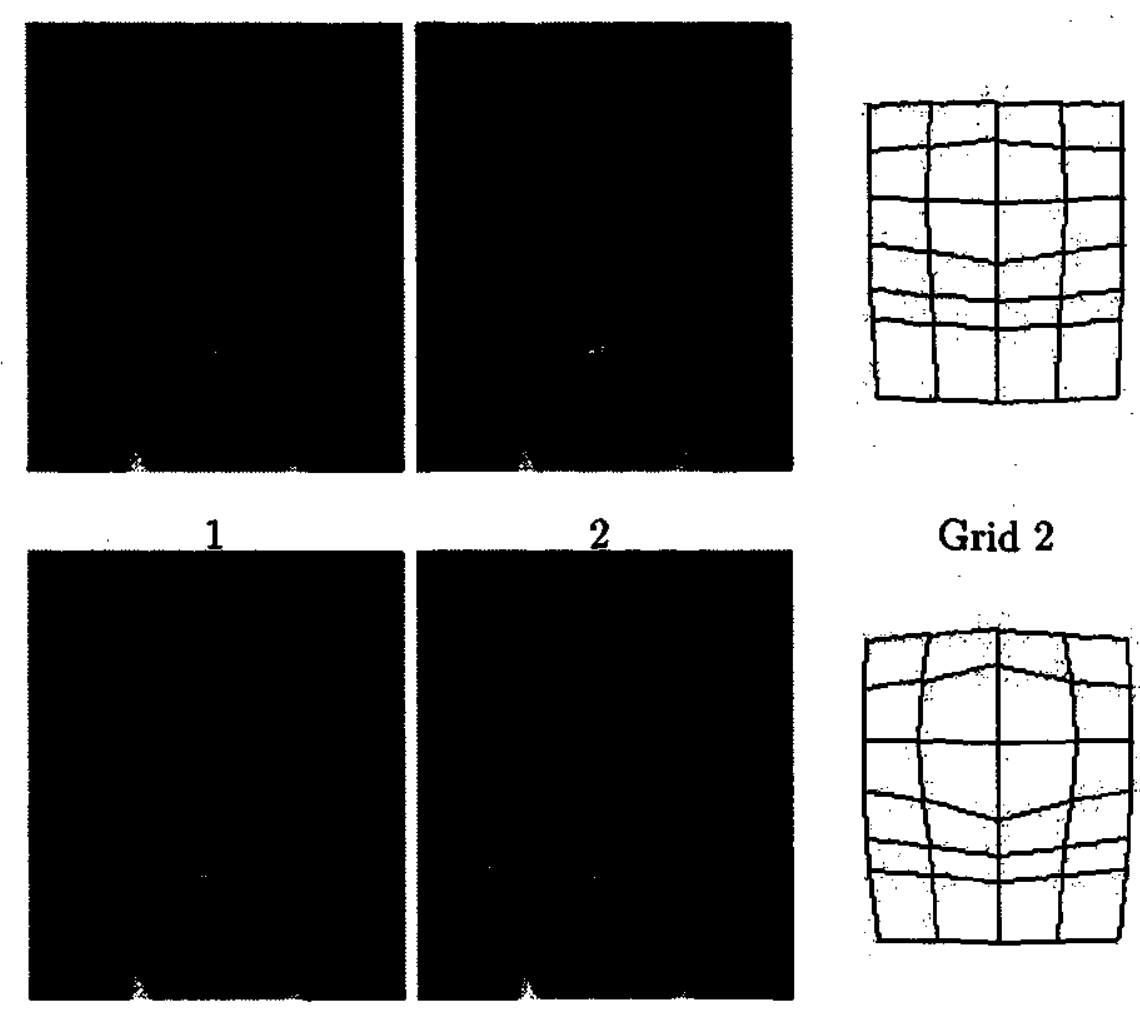

Grid 2
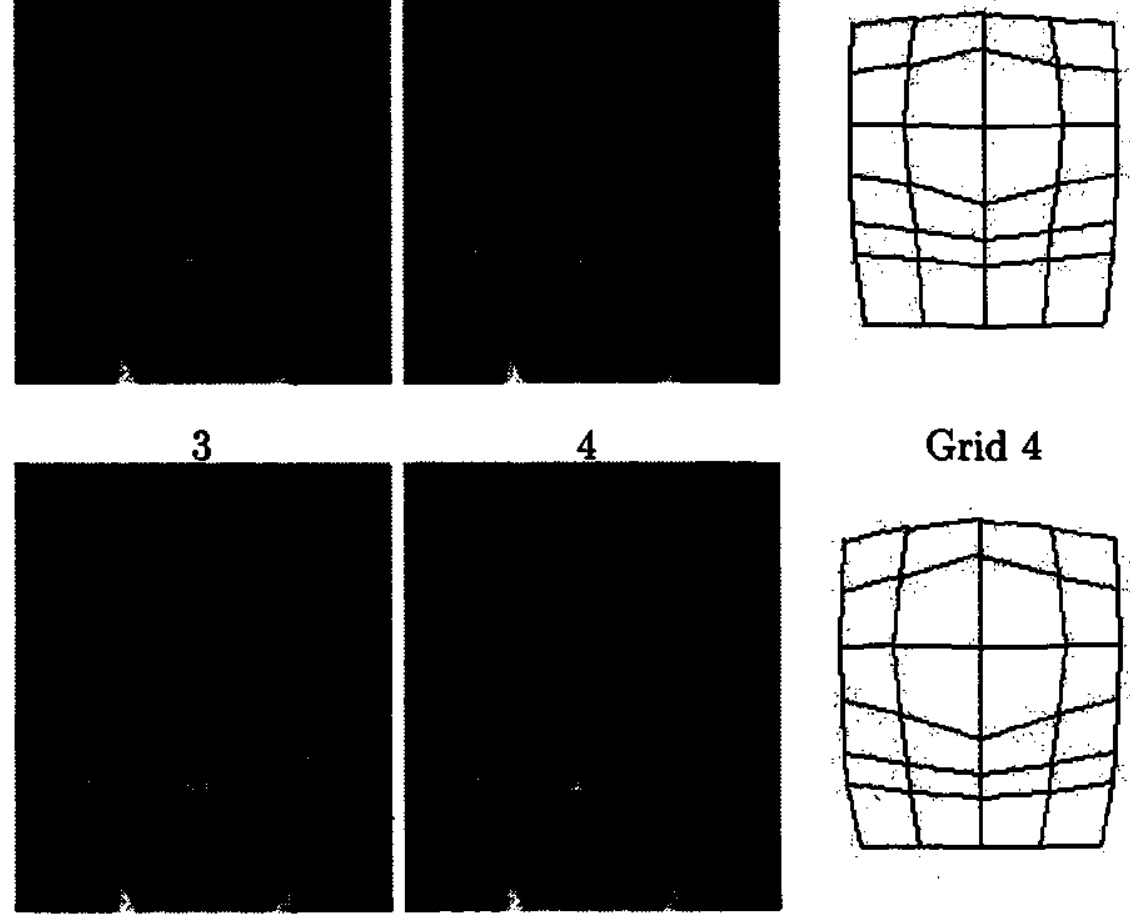

Grid 4

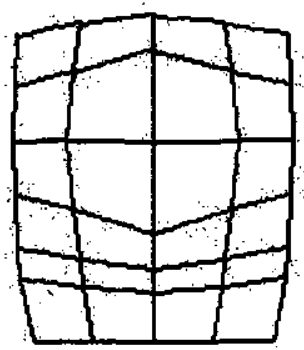

5

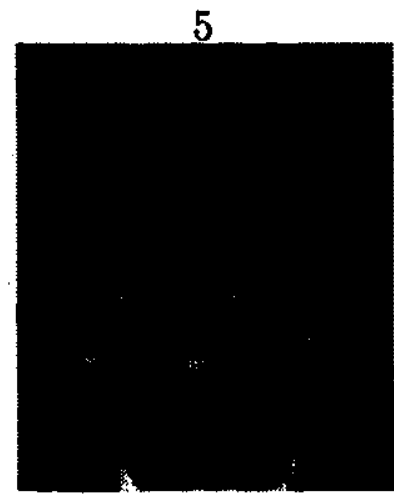

6

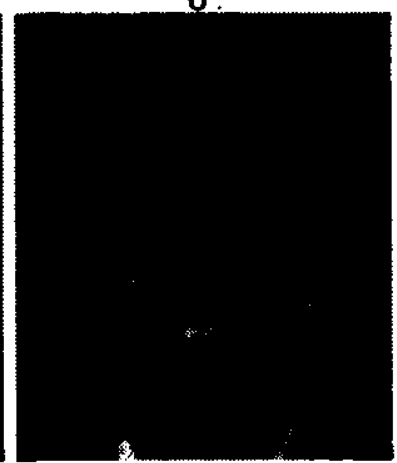

Grid 6

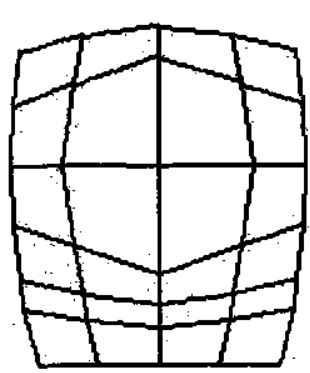

7

8

Figure 6.24 Mouth Movement for $n=1.5 \alpha=1.05$ and $\beta=$ 0.50 
The next figures, Fig. 0.5 and Fig. 0.6, show the ability to simulate a mouth in an opening and closing operation. The use of only four mass nodes can achieve full motion of a person talking, for more complicated expression more nodes have to be placed in the mouth. The ability to simulate the shrinking of facial objects is always easier to do because the bilinear warping method has more than enough pixels to fill the feature. A good example of this is Fig. 0.4, where the simulation of lips puckering is unique in the attracting of grid lines toward the center of the mouth.
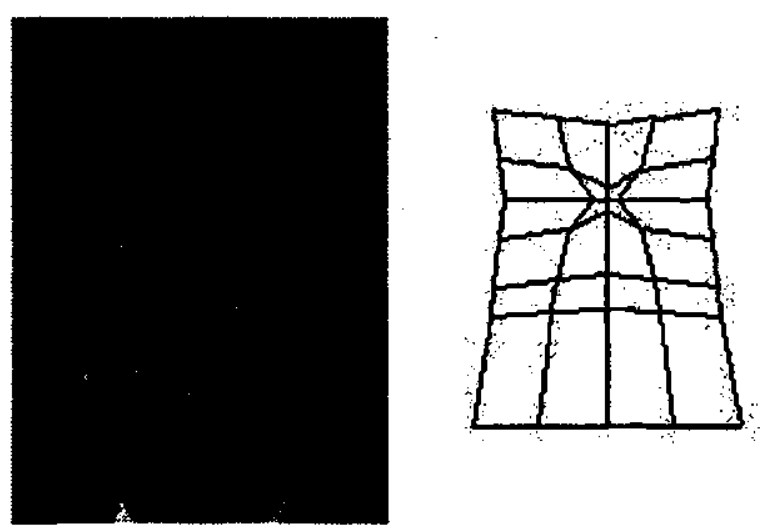

Figure 6.25 Lip Puckering 

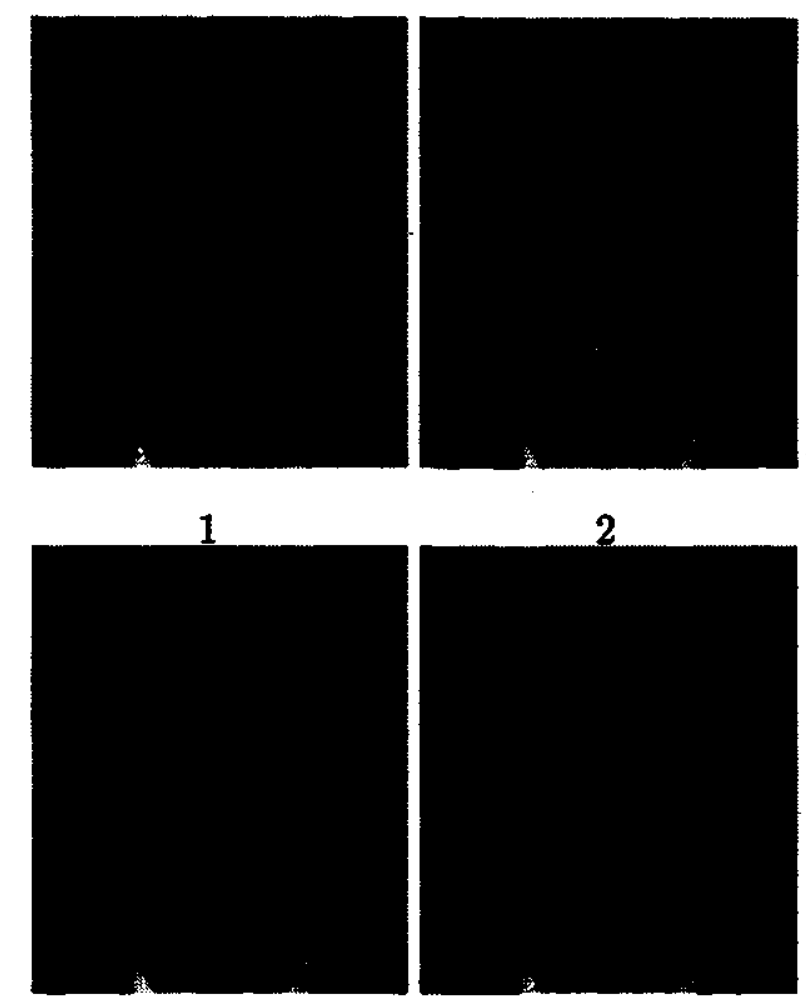

2

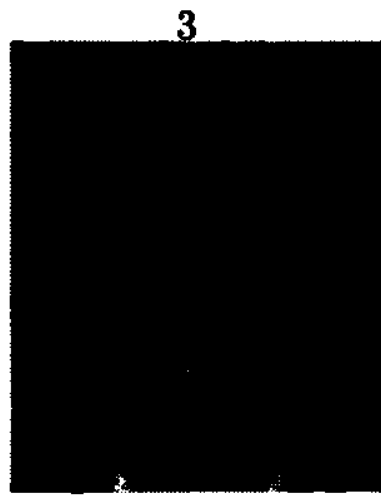

4
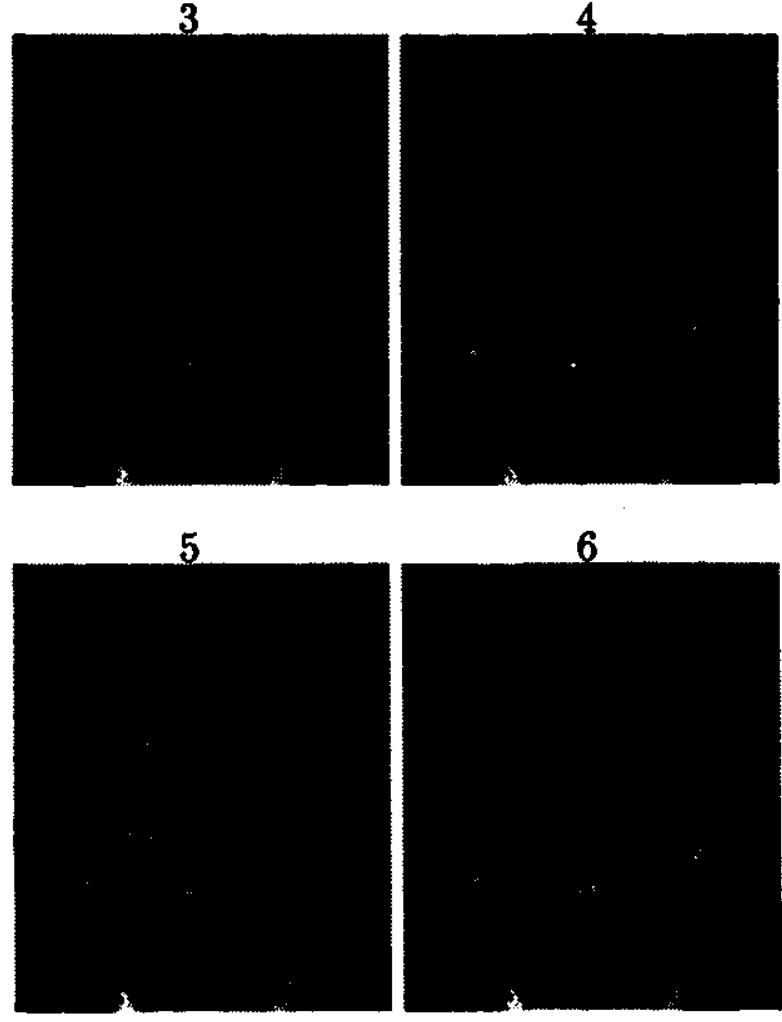

6

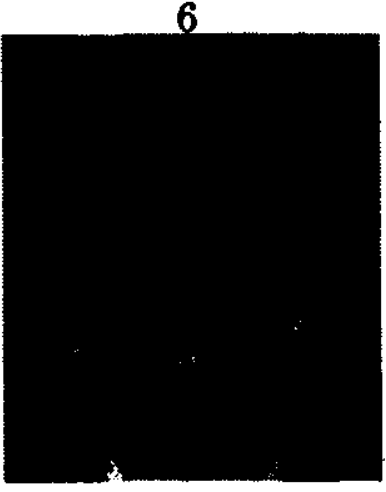

7

8

Figure 6.26 Typical Mouth Opening Movement 

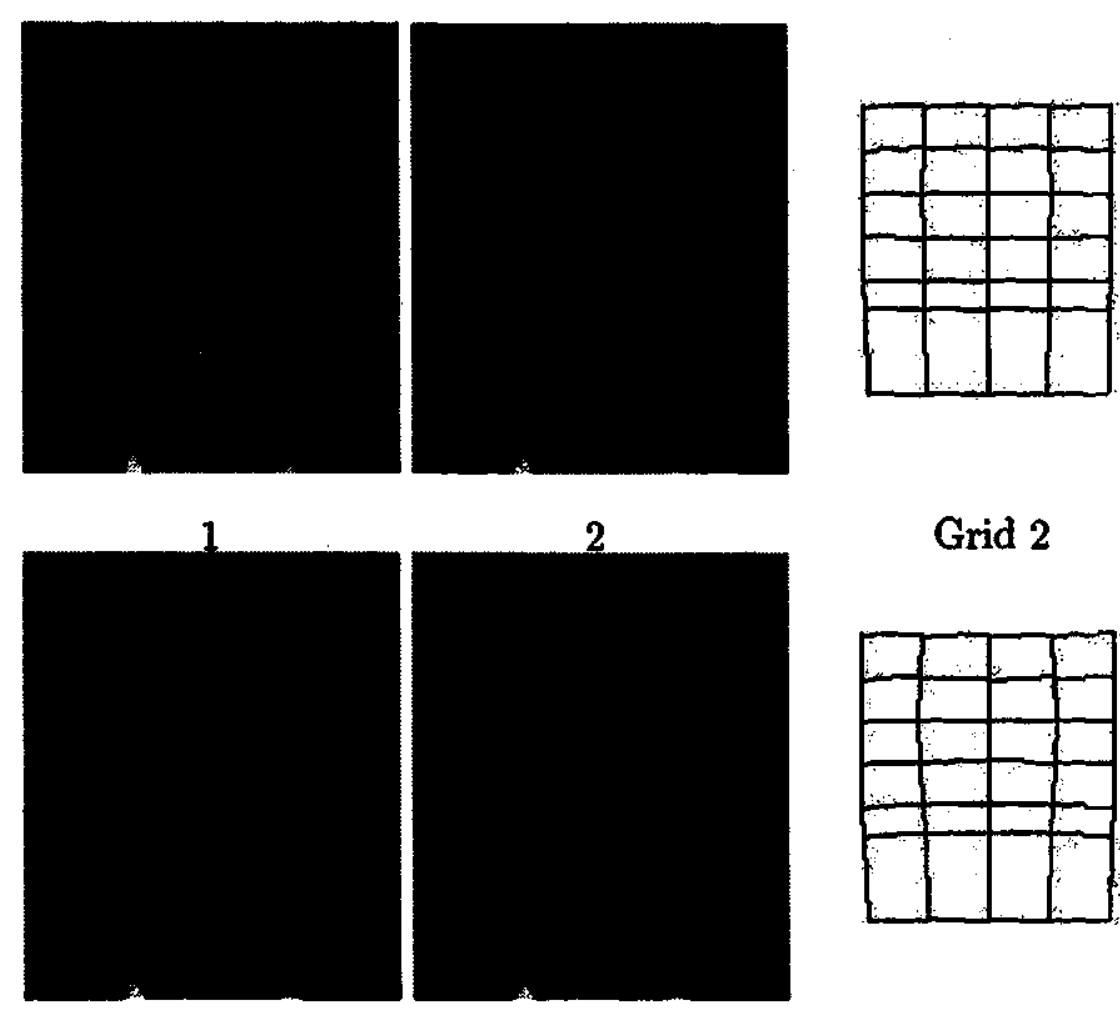

Grid 2
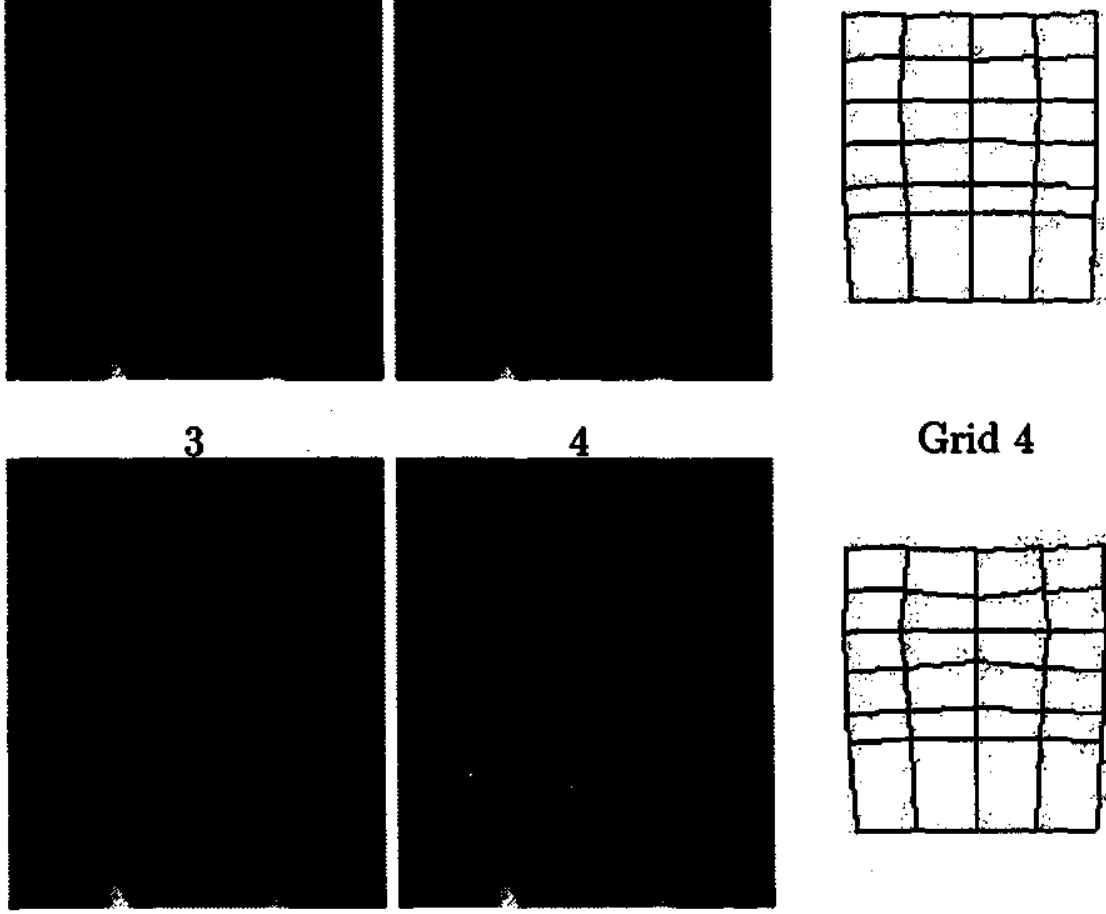

Grid 4

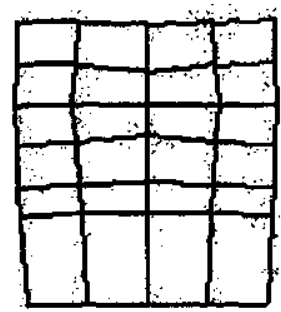

5

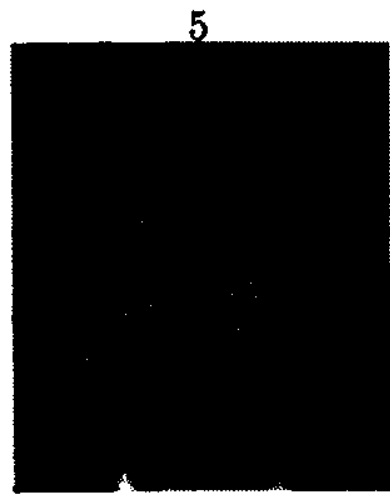

6

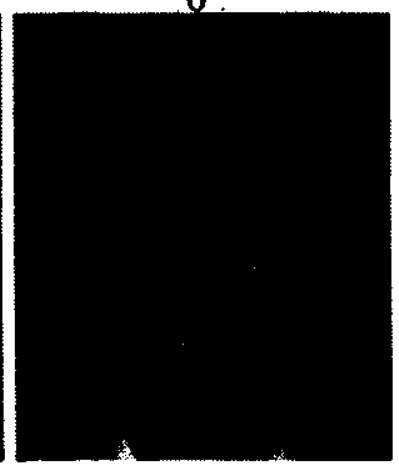

Grid 6

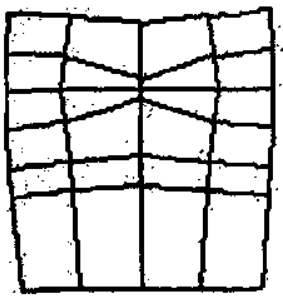

7

8

Grid 8

Figure 6.27 Typical Mouth Closing Movement 


\subsubsection{X-Z Rotation}

The use of the nonlinear movement of the grid lines in the head block is realized in Fig. 6.28, where the head is force to perform a right to left head rotation. The sides of the bounded head block and the middle line of the grid (Fig. 6.29) are used for the positions $x_{0}, x_{1}$, and $x_{i}$ (as indicated in Eq. 4.13). $n$ is set to two to represent a second order function. The grid lines are pulled from right to left giving the appearance of facial features moving in the direction of motion. 

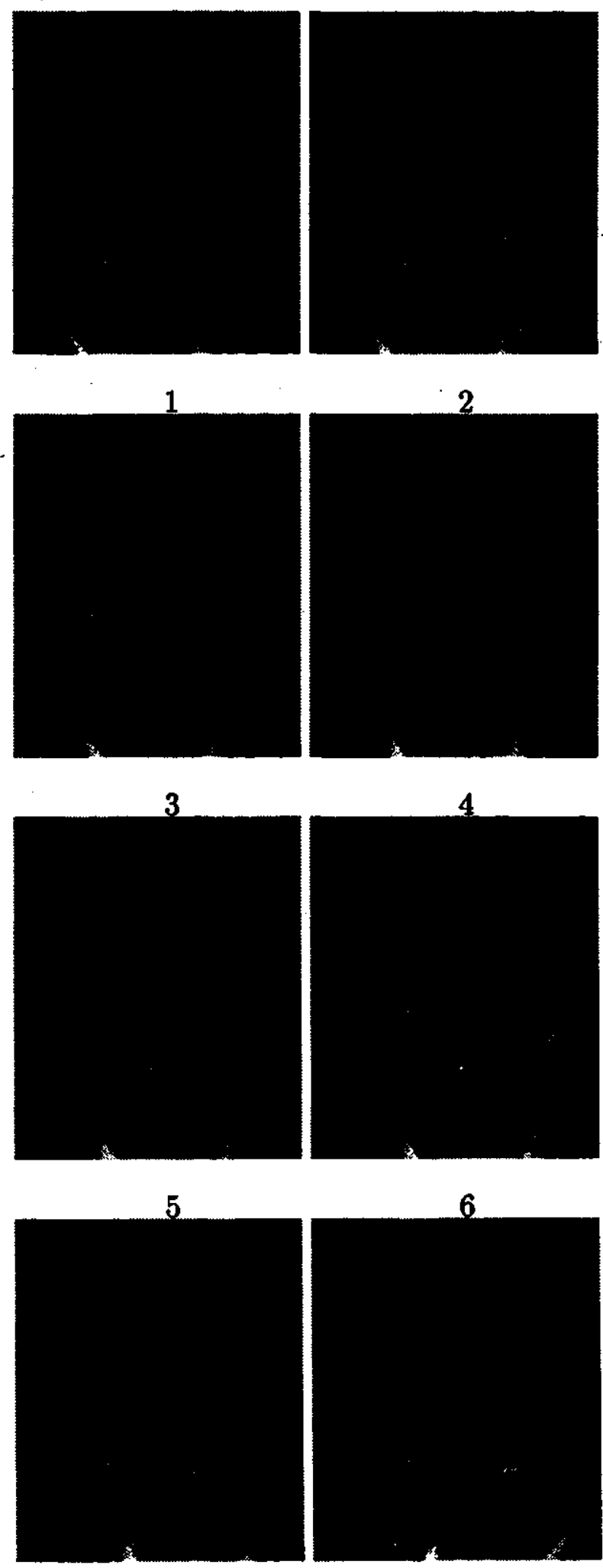

7

8

Figure 6.28 X-Z Head Rotation Sequence 

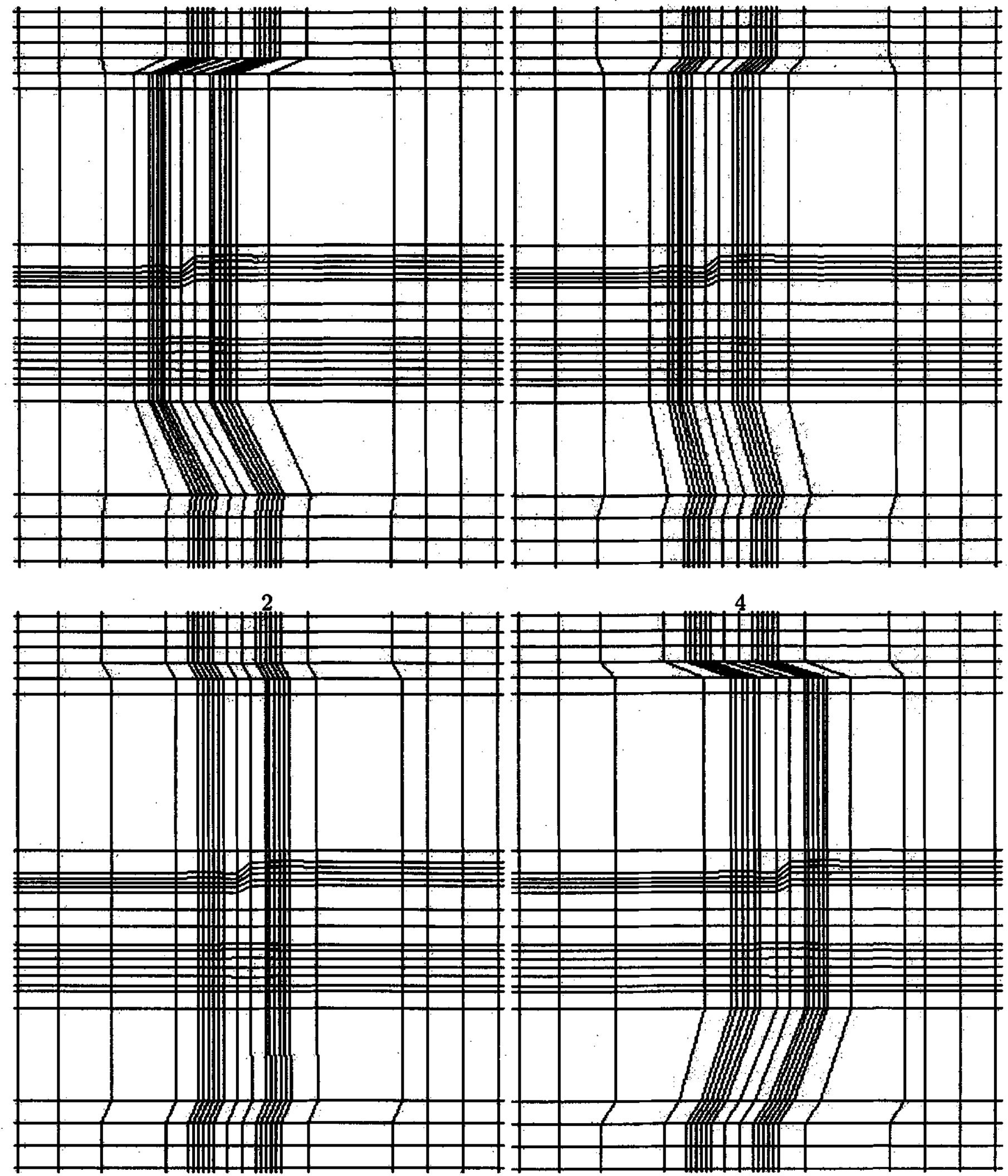

6

8

Figure 6.29 X-Z Head Rotation Grids 


\subsubsection{Y-Z Rotation}

An example of how this simulated Y-Z motion would appear is shown in Fig. 6.30. The fixed boundaries are set to the bottom and top of the head block, and the reference position is the middle line in the block close to the nose. The effect is the nonlinear pulling of the head block's vertical lines in the direction of motion as shown in Fig. 6.31. This image approximates a nodding motion of the head with the widening and shrinking of features. 

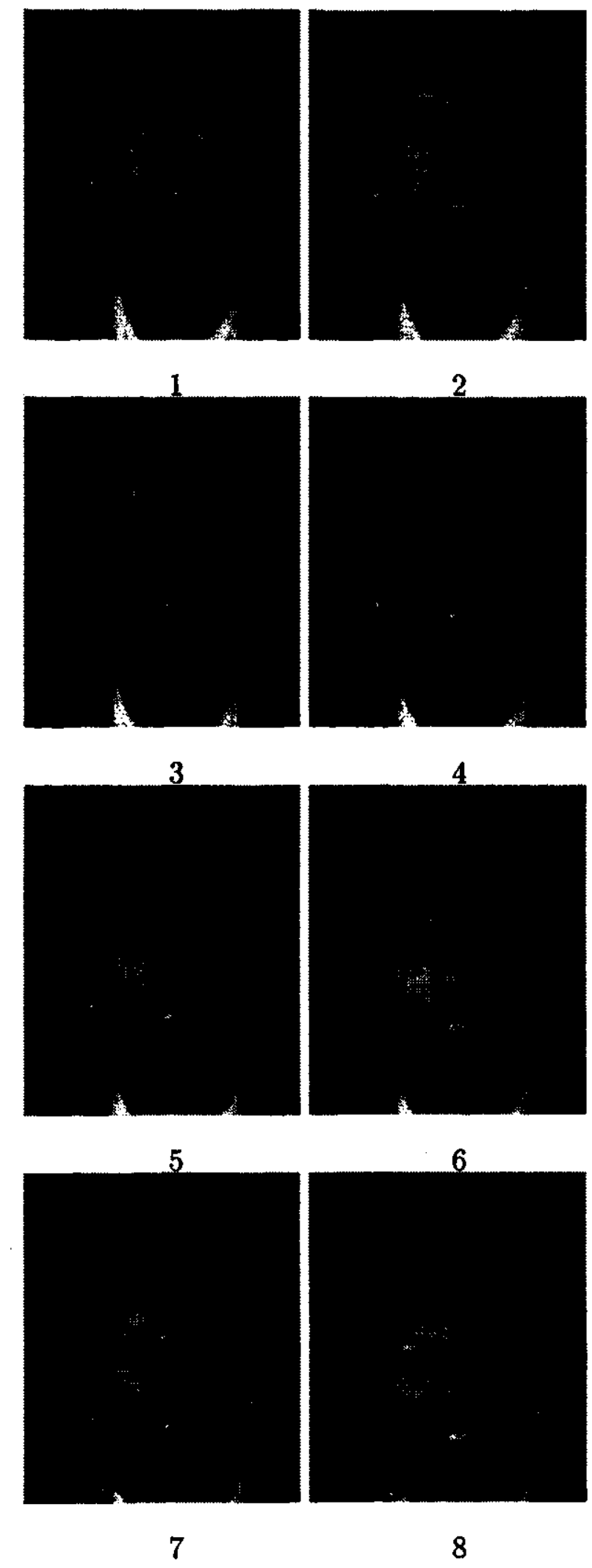

8

Figure 6.30 Y-Z Head Rotation Sequence 

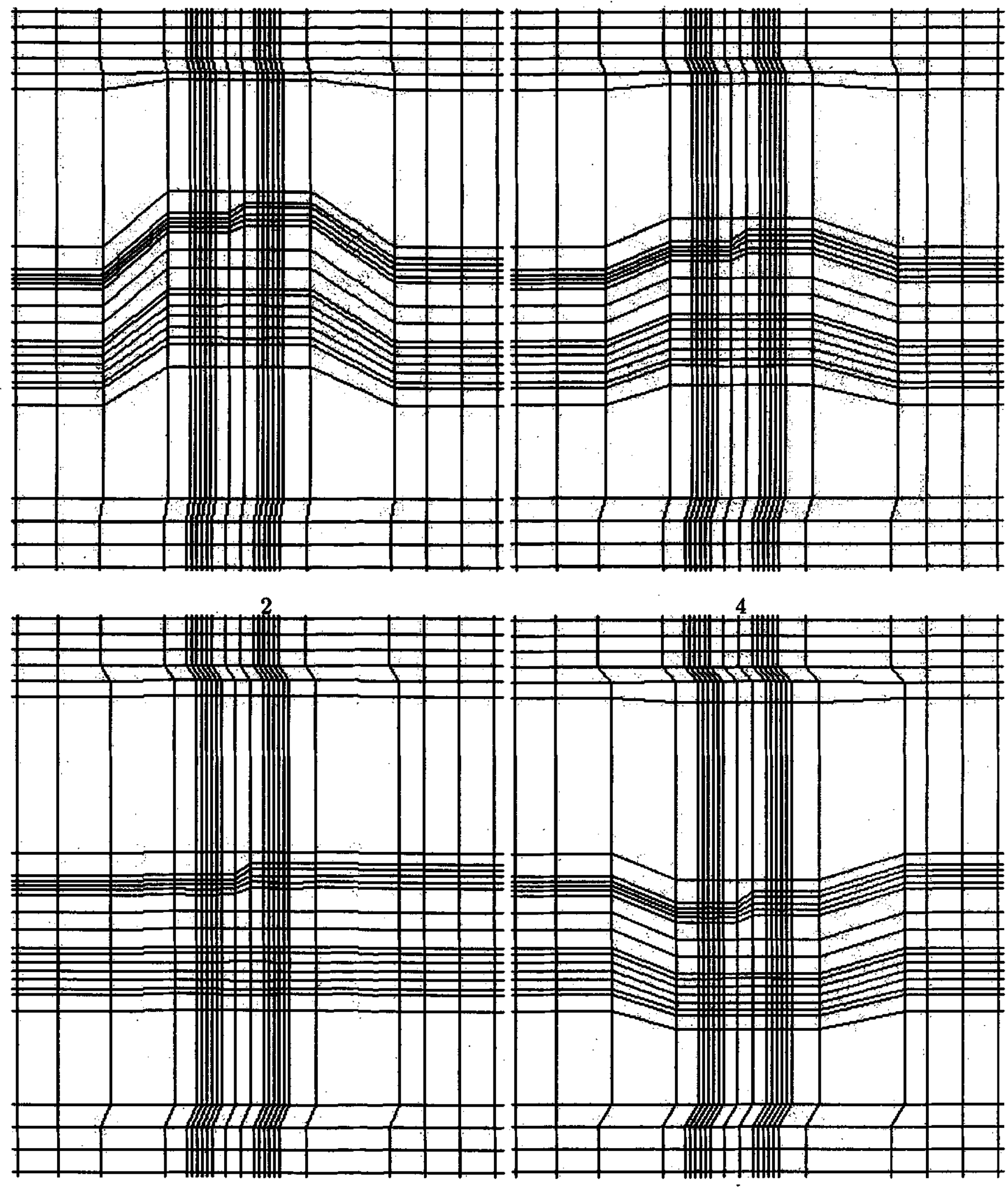

6

Figure 6.31 Y-Z Head Rotation Grids 


\subsubsection{X-Y Rotation}

The movement of a head from side to side uses a pivot at the neck area. The amount of angular movement is limited to the source image. Too much movement can result in anomalies showing up in the image. In Fig. 6.32, a sequence of eight frames are shown with the movement of the head grid for every other frame. The head is forced to move counterclockwise with one degree of motion per frame occurring, the movement of one degree can cause significant motion to occur. At the $4^{\circ}$ point the squareness of the head block becomes apparent as indicated in the shoulders. However, at the $8^{\circ}$ mark the range of head motion would look unnatural. In Fig. 6.34, the movement in the clockwise direction is limited by the same amount of rotation. 

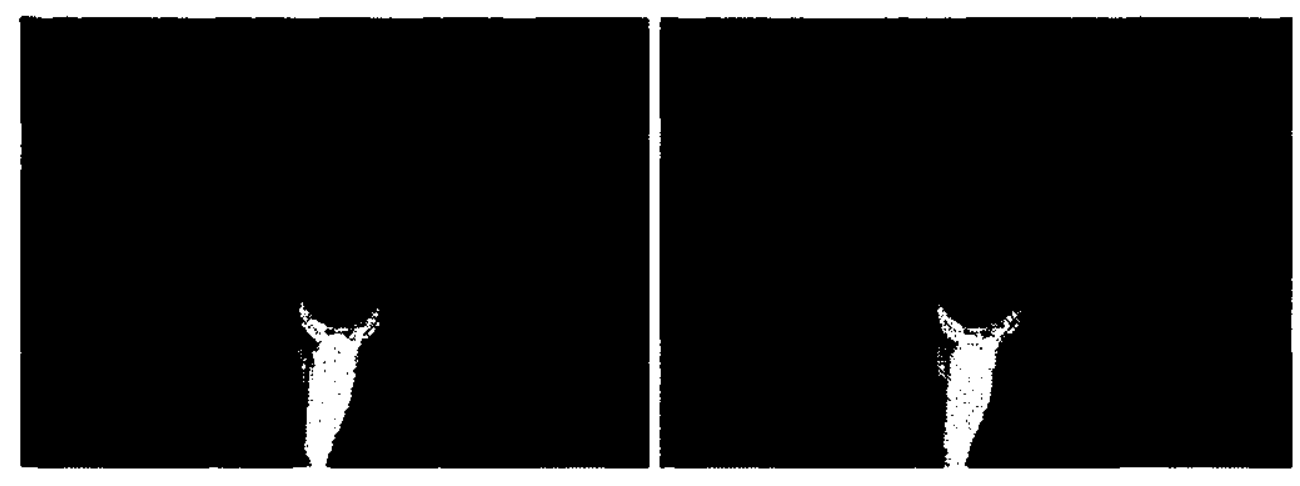

1

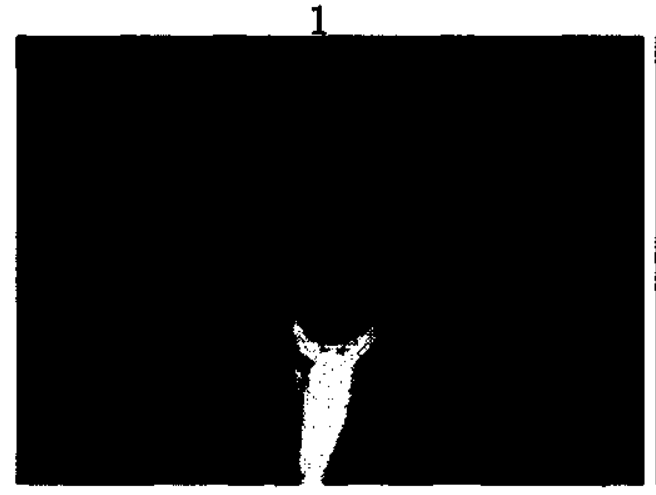

3

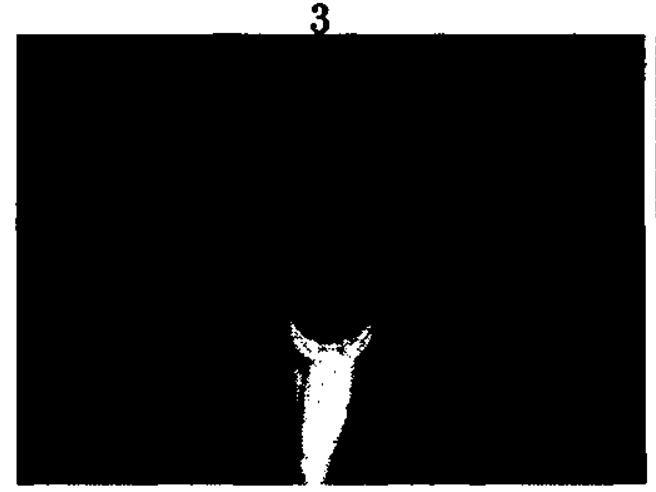

5

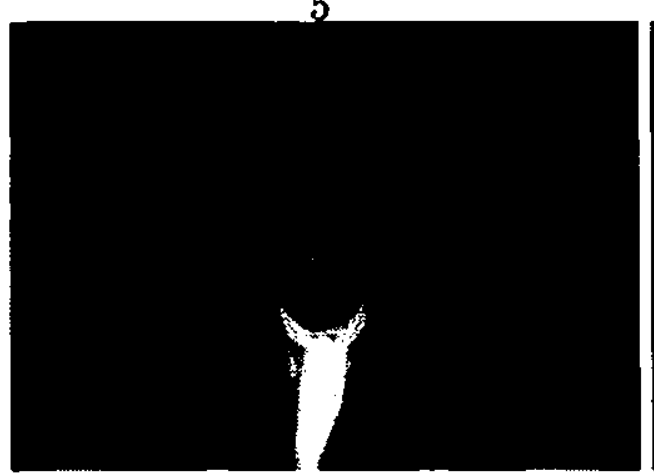

7
2

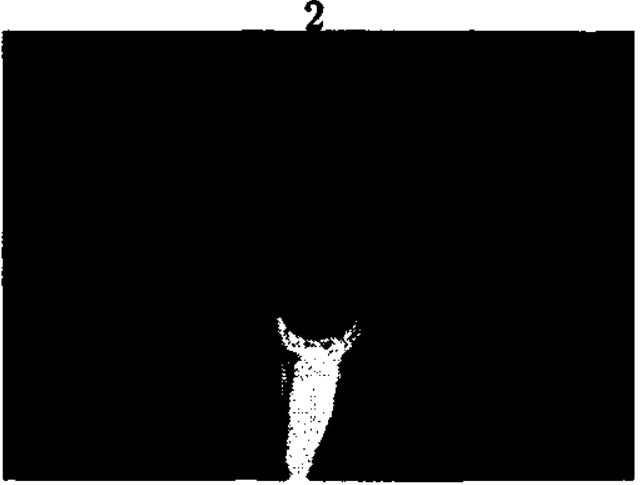

4

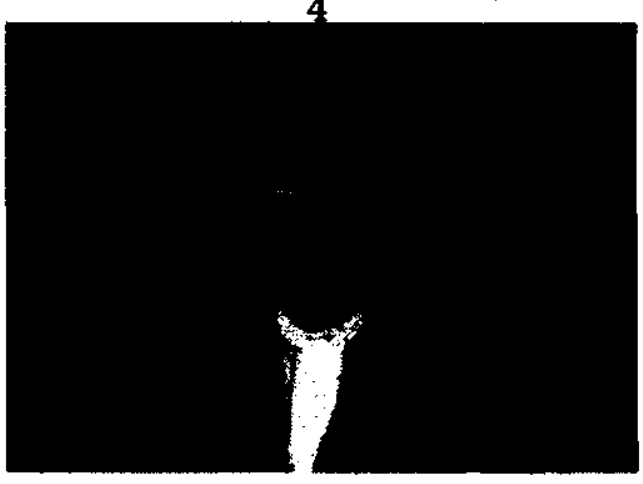

6

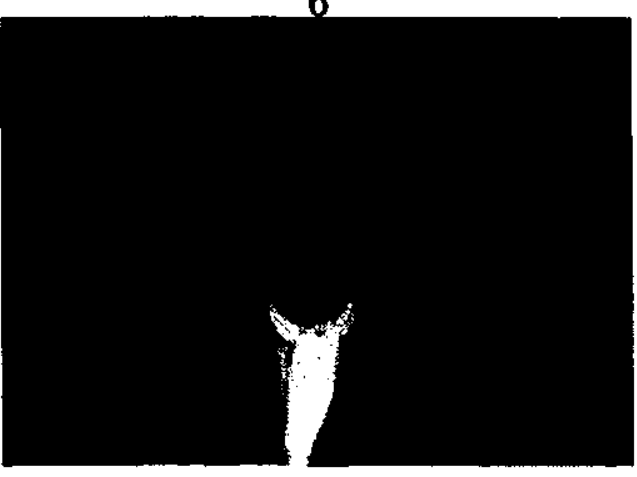

8

Figure 6.32 X-Y C.C.W Rotation, $\theta=1^{\circ}$ per frame 


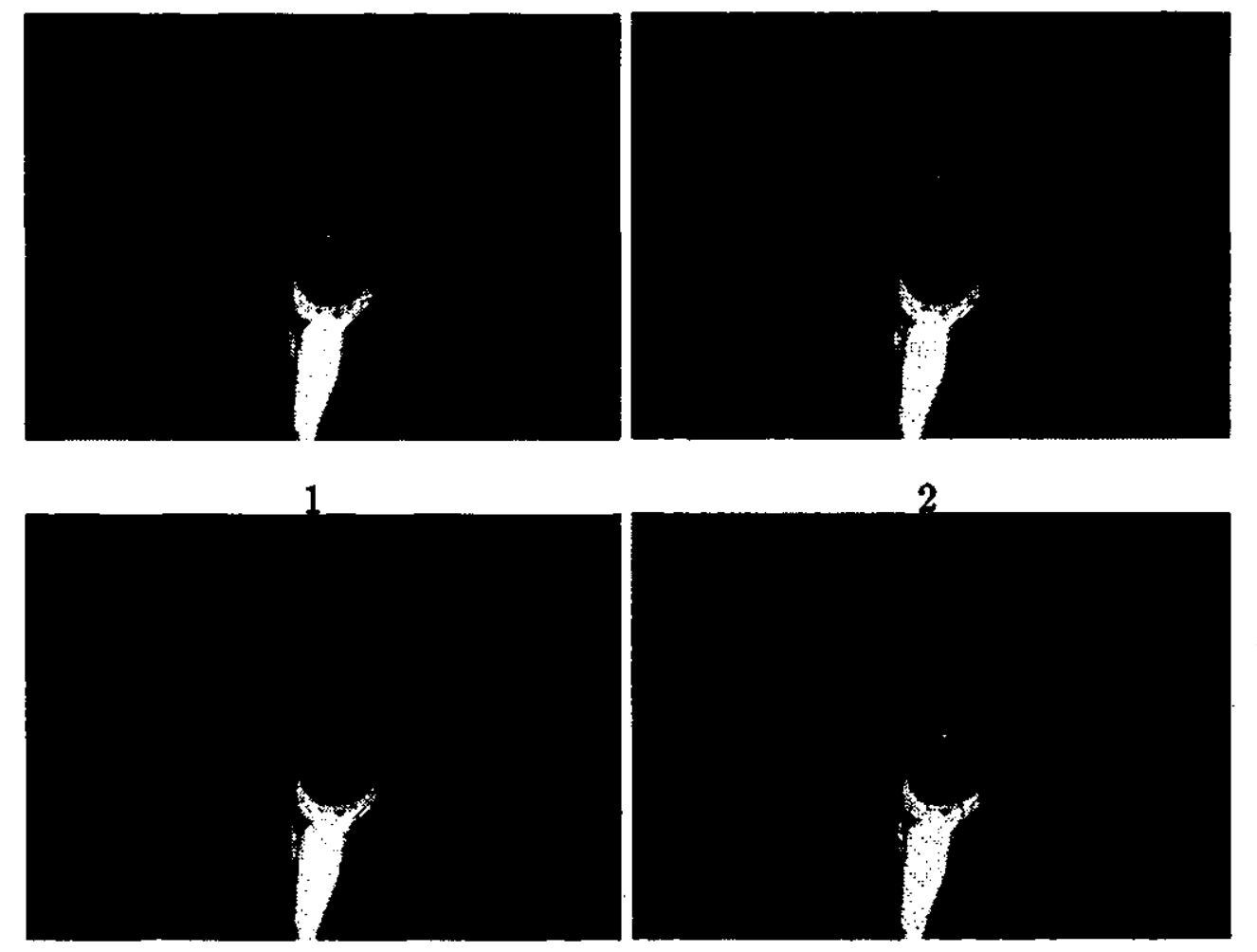

3

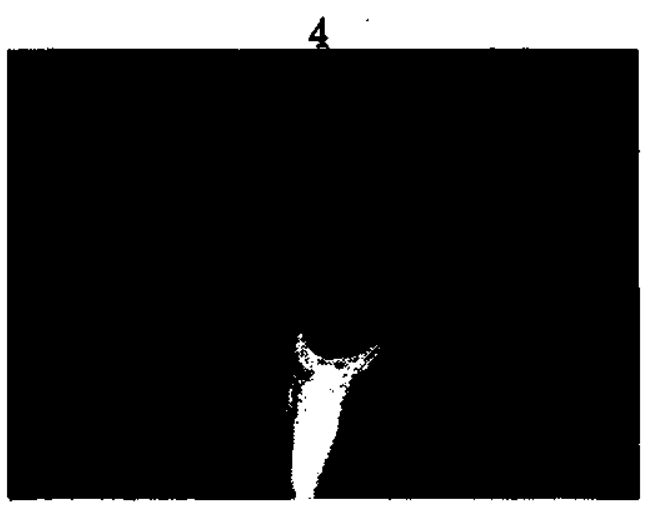

5

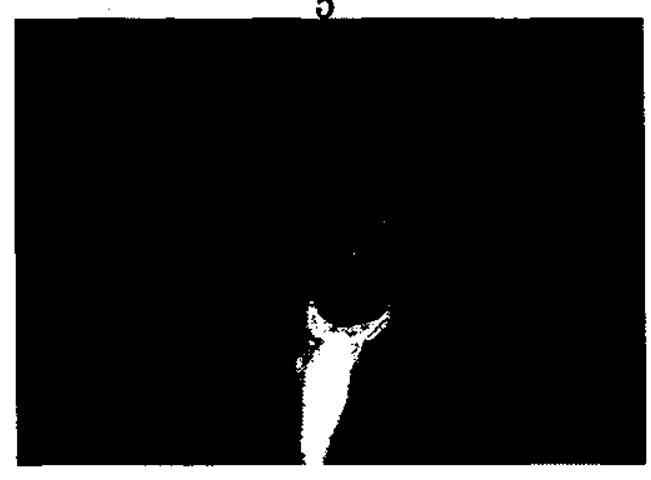

6

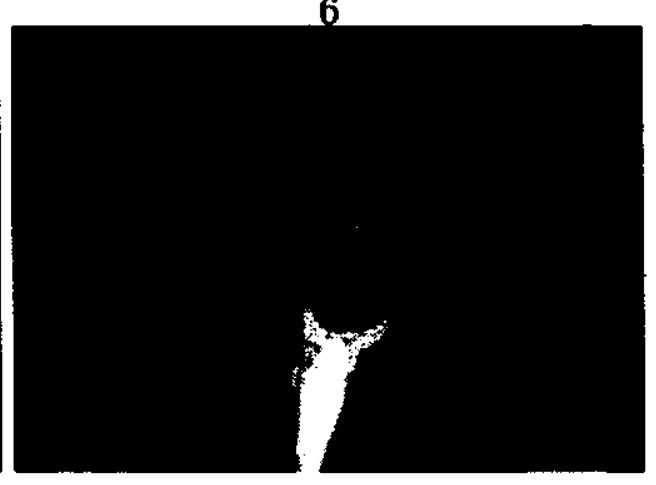

7

8

Figure 6.34 X-Y C.W Rotation, $\theta=1^{\circ}$ per frame 


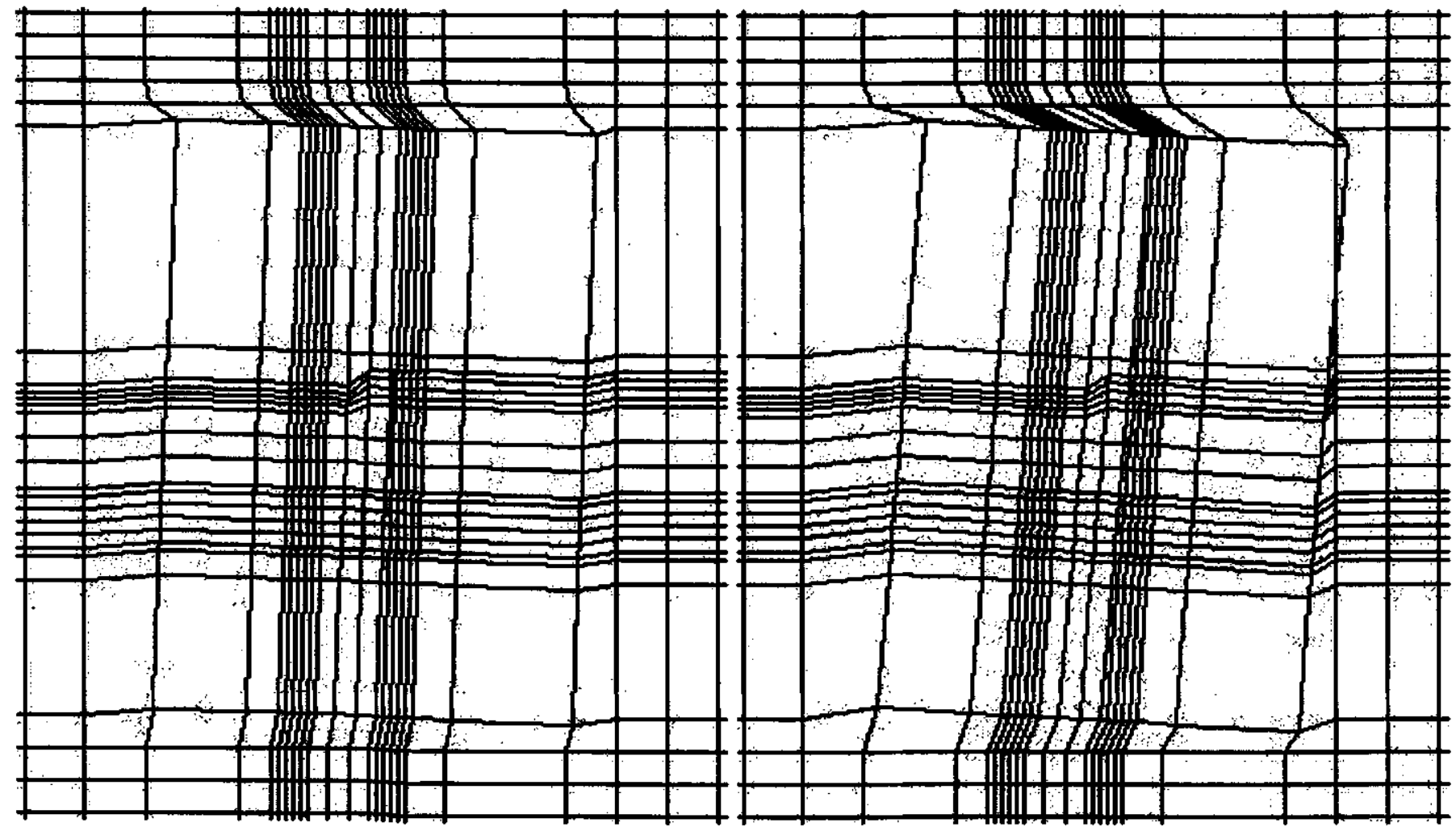

2

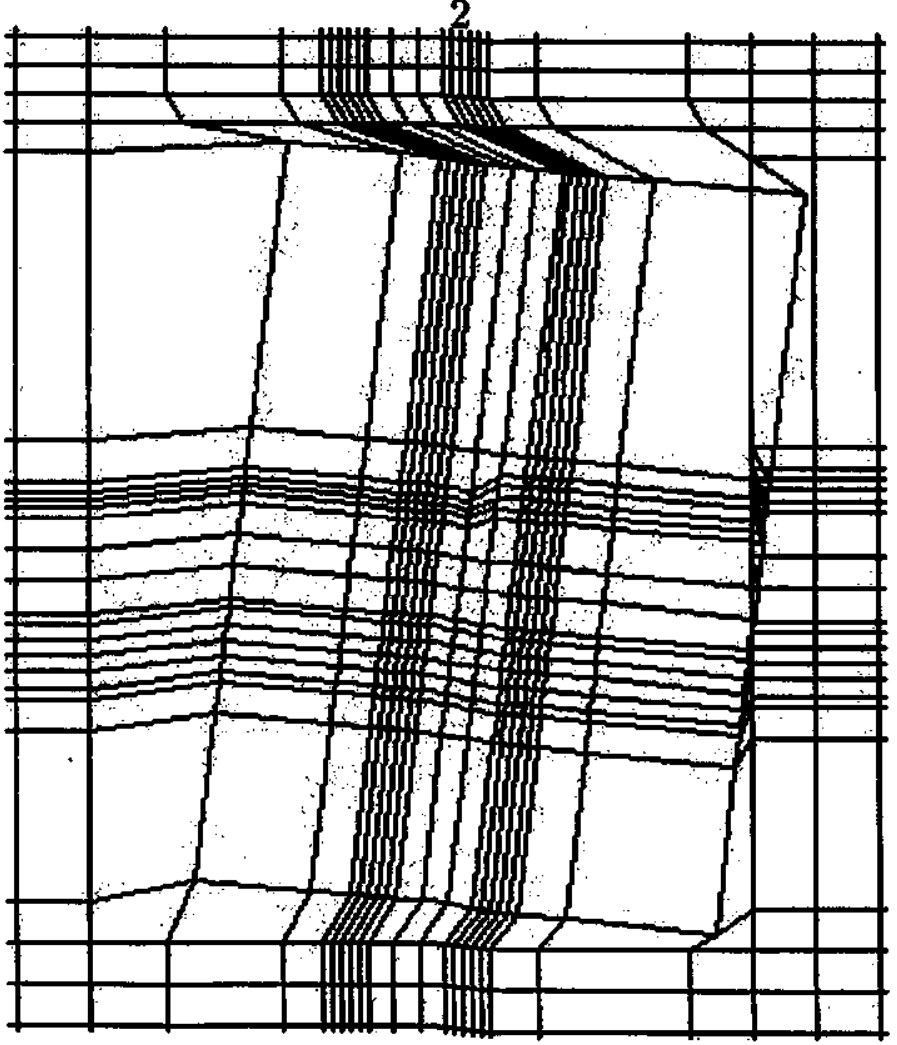

6

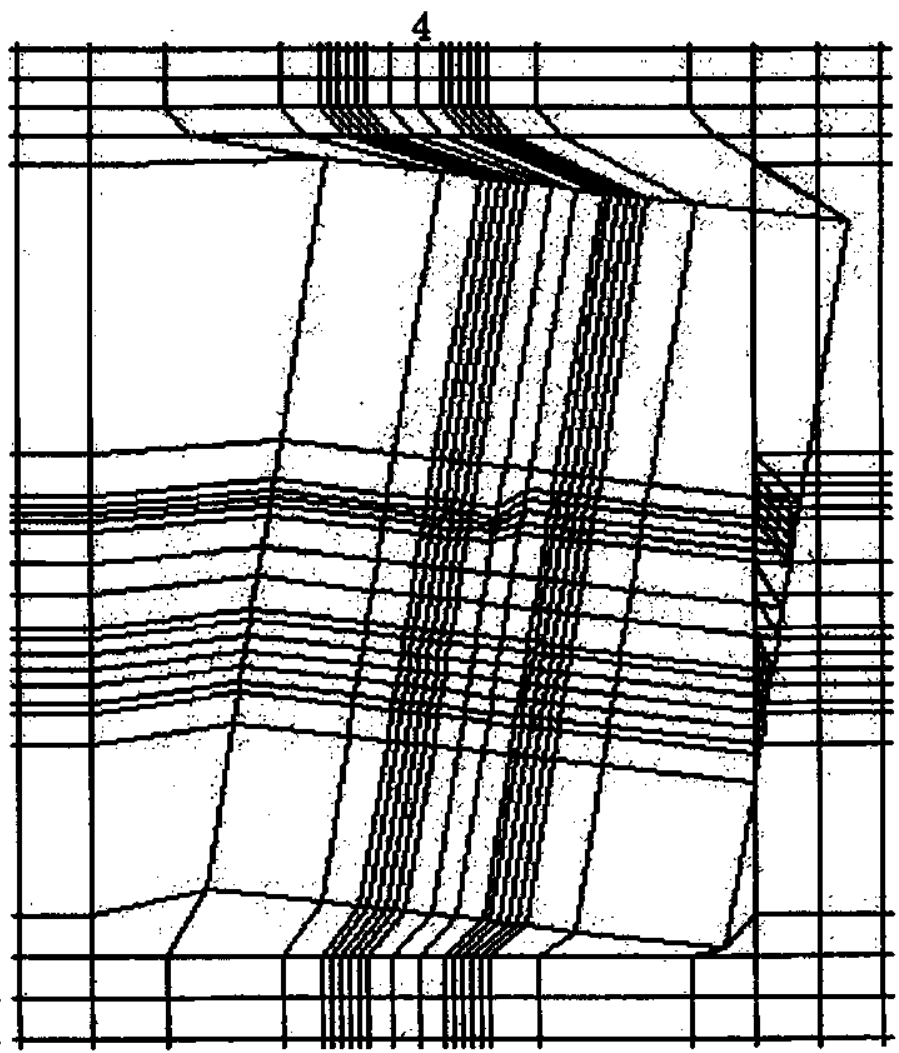

8

Figure 6.35 X-Y C.W Rotation Grids, $\theta=2^{\circ}$ per frame 


\subsection{Encoder Testing}

The extent of the work for the encoder will only focus on the ability to measure facial geometry for successive video frames. These measurements present the information of how objects in the face move and how head rotation can be interpreted. To test the facial extraction the database consists of a sequence of $\mathbf{9 0}$ frames of the Claire video standard. The sequence shows the woman Claire talking to the camera.

The program was written to log all the results of facial measurements in one pass of the database. In order for the program to work, each frame must pass three extraction algorithms. First, the head block is extracted from the image using Average Amplitude Projection. Then edge detection filters (Robinson template gradient) extract the edge information of the face. If these methods are successful then the outer edge of the head is extracted. For the database tested, the extraction methods were consistent in all 90 frames.

The structure of this program requires that each frame pass head extraction, and edge extraction before finding facial features. Chapter 5 covers the topics of Average Amplitude Projection for head extraction, and the use of Gradient and Laplacian filters for edge extraction. Though no testing was performed for these subroutines, both methods were capable in completing their objectives. Extraction of the head boundary was not tested either due to the homogeneous background of the Claire video clip.

The extraction of the objects in the face can use an adaptive method because the Claire's video sequence was recorded at $30 \mathrm{fps}$. This means objects detected in the face will move relatively short distance from frame to frame. The figures that follow show the ability to track objects like the eyes, nose, and mouth. If the correct objects are found then measurements of these objects are straightforward. The sequence of images was repeatedly ran to insure the ability of the encoder. On average, the extracted objects were found 80 percent of the time. Most noticeable failures that occur are related to edge detection in the eyes, mouth, and nose. These errors have 
been replaced with adjacent frame values so that the figures can show what type of movement had occurred. For the errors that remain, movement of five or more pixels in a single frame may be considered an incorrect result.

The first three graphs, Fig. 6.36, Fig. 6.37, and Fig. 6.38, show the movements of the eyes' and mouth's horizontal and vertical width changes. Such movements reflect the types of information that would apply to the use of the $A / R$ in the decoder. In Fig. 6.36 it can be deduced from the graph that the vertical width of the eyes varies from 5 to 15 pixels with a maximum change of no more that 5 pixels. Also, this particular video clip both eyes simultaneously do the same action. The right eye accurateness is less than the left eye due to the larger positional variations. In Fig. 6.37 the horizontal lengths of the eyes are fairly constant over the sequence, which was anticipated.

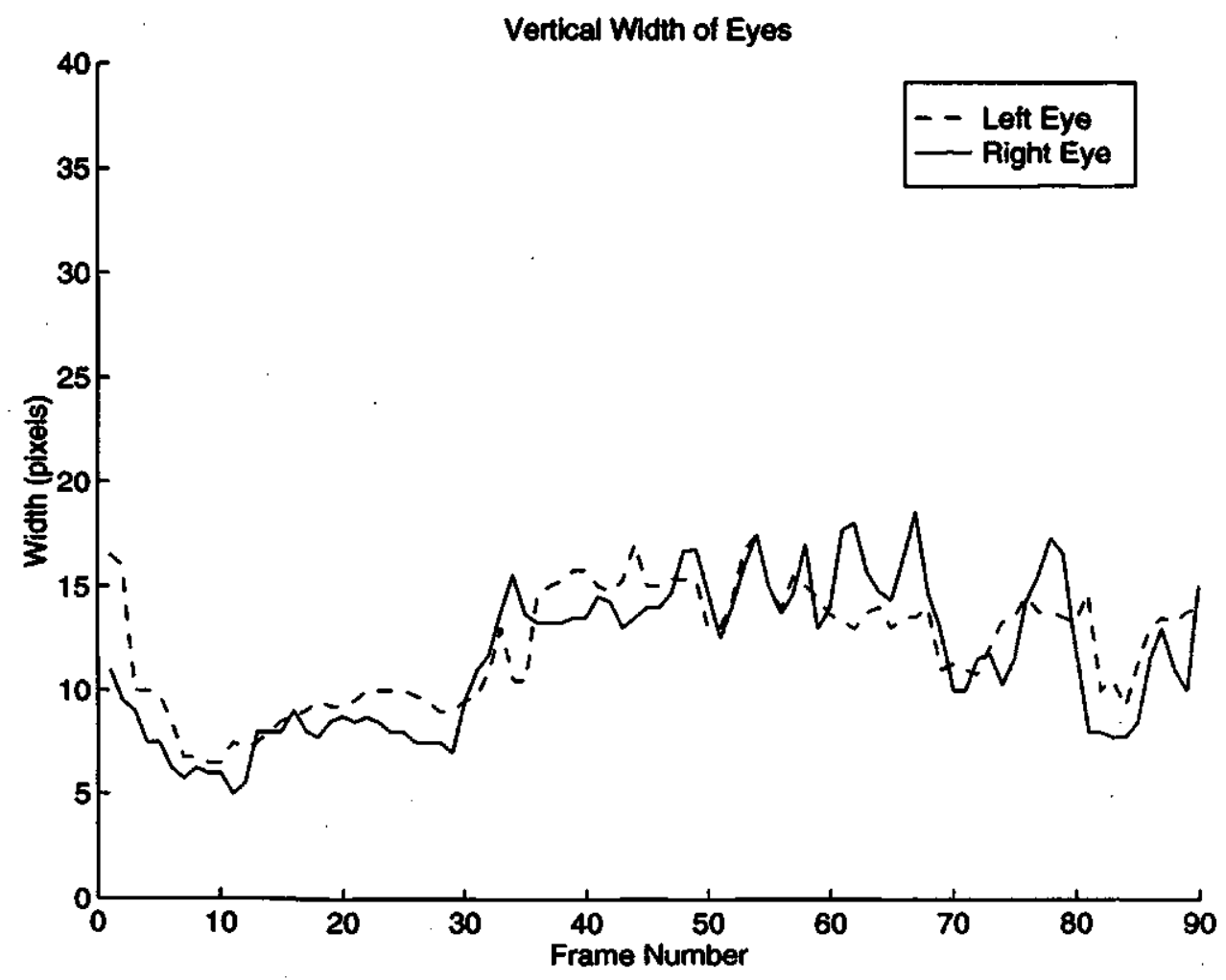

Figure 6.36 Tracking Eye Vertical Movement 


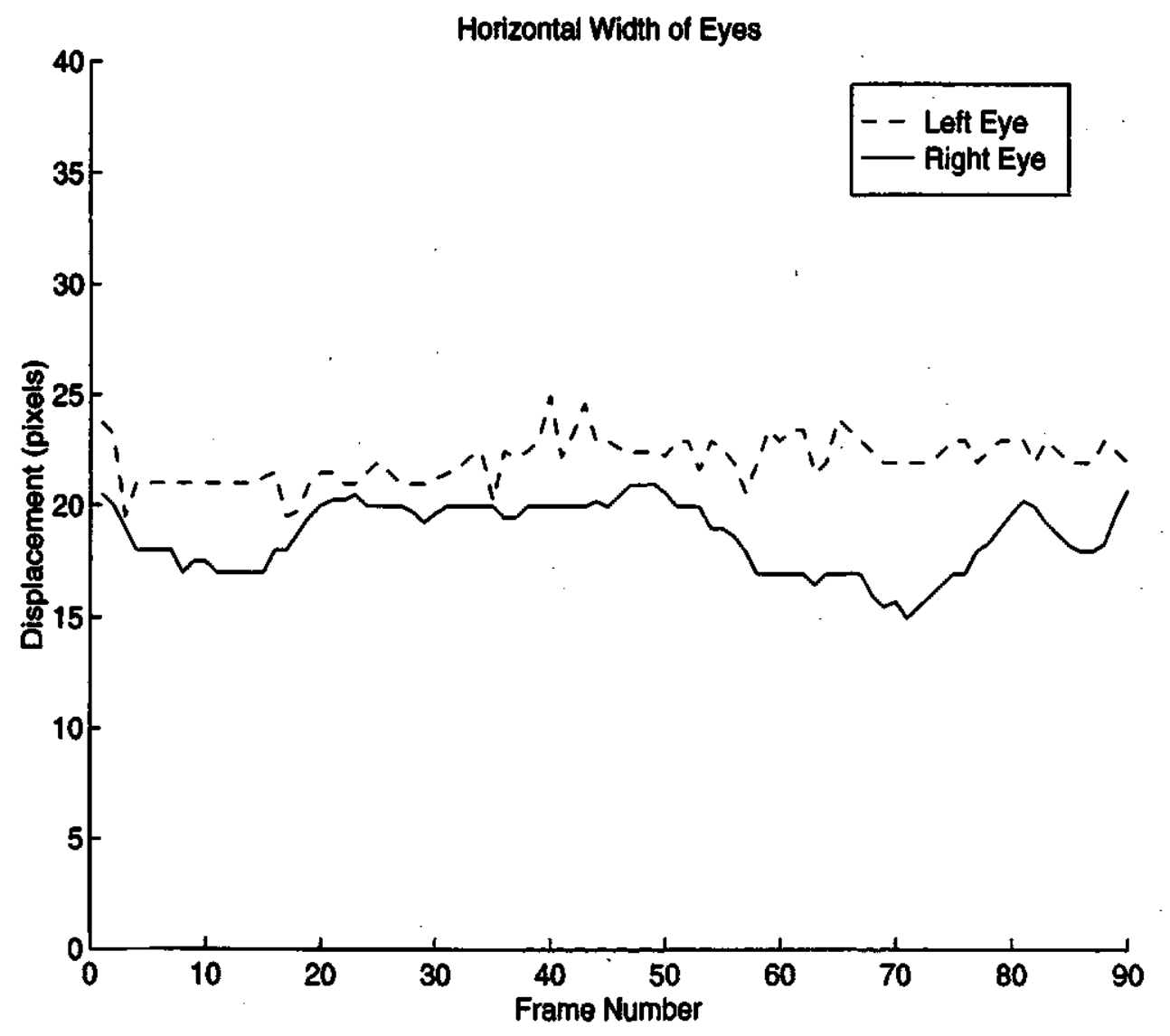

Figure 6.37 Tracking Eye Horizontal Movement 
As shown in Fig. 6.38, the width and length of the mouth are both expected to have variations in motion. In the sequence, the width and length of the mouth tended to increase and decrease in size at the same times. Thus verifying the need for $\beta$ and $\alpha$ terms in the Attractor/Repellor decoding algorithm.

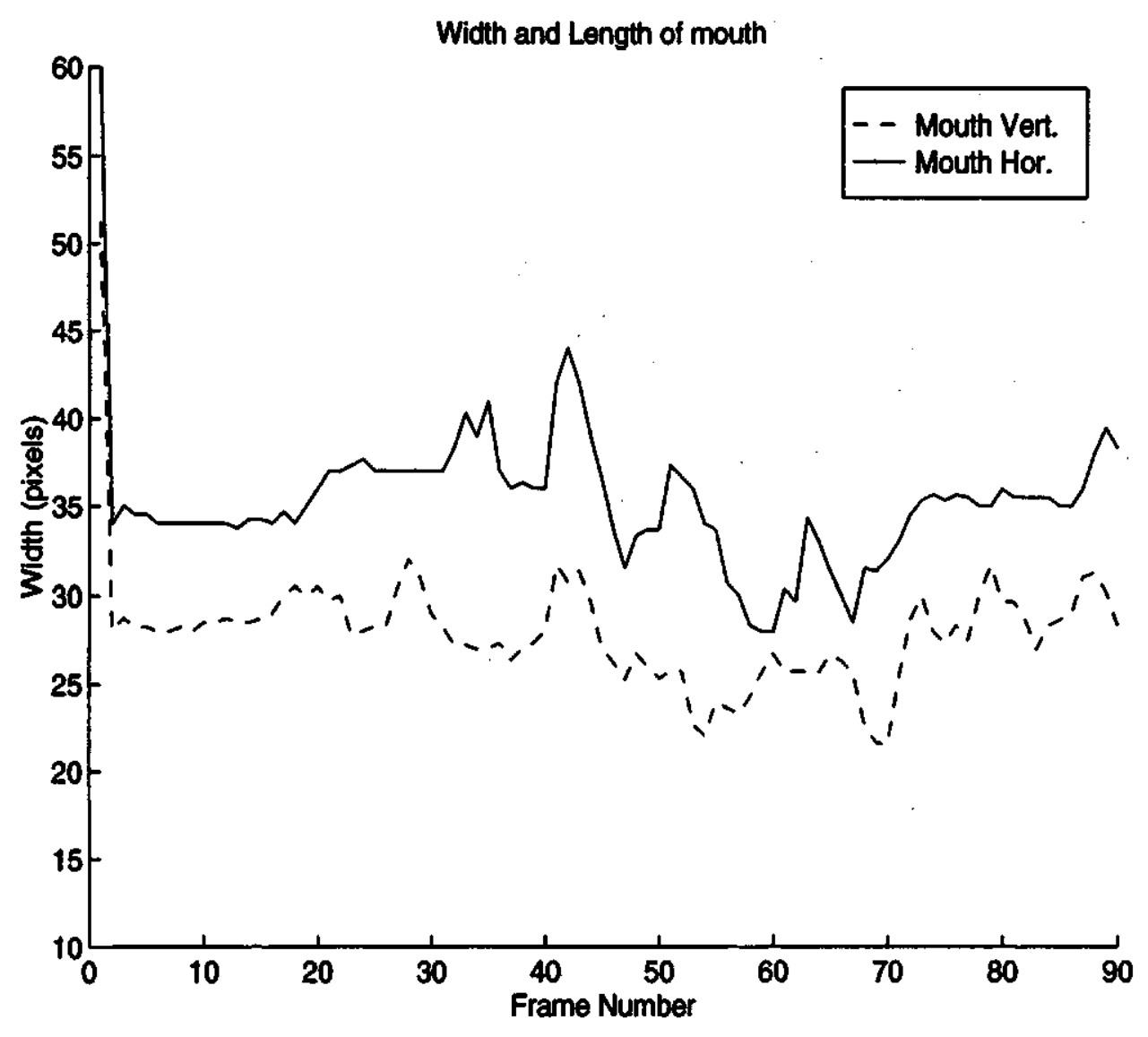

Figure 6.38 Tracking of Mouth Movements 


\subsubsection{Head Rotation Interpretation}

Exact three dimensional head motion is difficult at best to find from a two dimensional picture. Since the object is a person's face, certain assumptions can be made about the relative motion of the facial objects in each of the $\mathrm{X}-\mathrm{Z}$ and $\mathrm{Y}-\mathrm{Z}$ planes. Various distances among objects can be measured from frame to frame to find the action.

$\mathrm{X}-\mathrm{Z}$ motion is generally easier to detect than $\mathrm{Y}-\mathrm{Z}$ motion because of the method of object extraction use horizontal scanning techniques to extract the inside edges of the face. To measure $\mathrm{X}-\mathrm{Z}$ motion the three points that conform to the $\mathrm{X}-\mathrm{Z}$ decoder method are the nose, and two points on either side of the face directly across from the nose. The two points that make up the sides of the head can be considered as boundaries for the nose.

Fig. 6.39 shows the width of the face changes very little, with a maximum movement of 5 pixels. This measurement alone cannot tell if any head rotation has occurred, however it does establish that head boundaries should remain constant for small X-Z rotations. Fig. 6.40 shows that the distance between the nose and the left side of the face decreases, while the distance between the nose and the right side of the face increases. These graphs clearly indicate that the head did perform an X-Z rotation toward the left. Thus, the three-point method of nonlinear scaling used by the decoder can be successfully used with the encoder. 


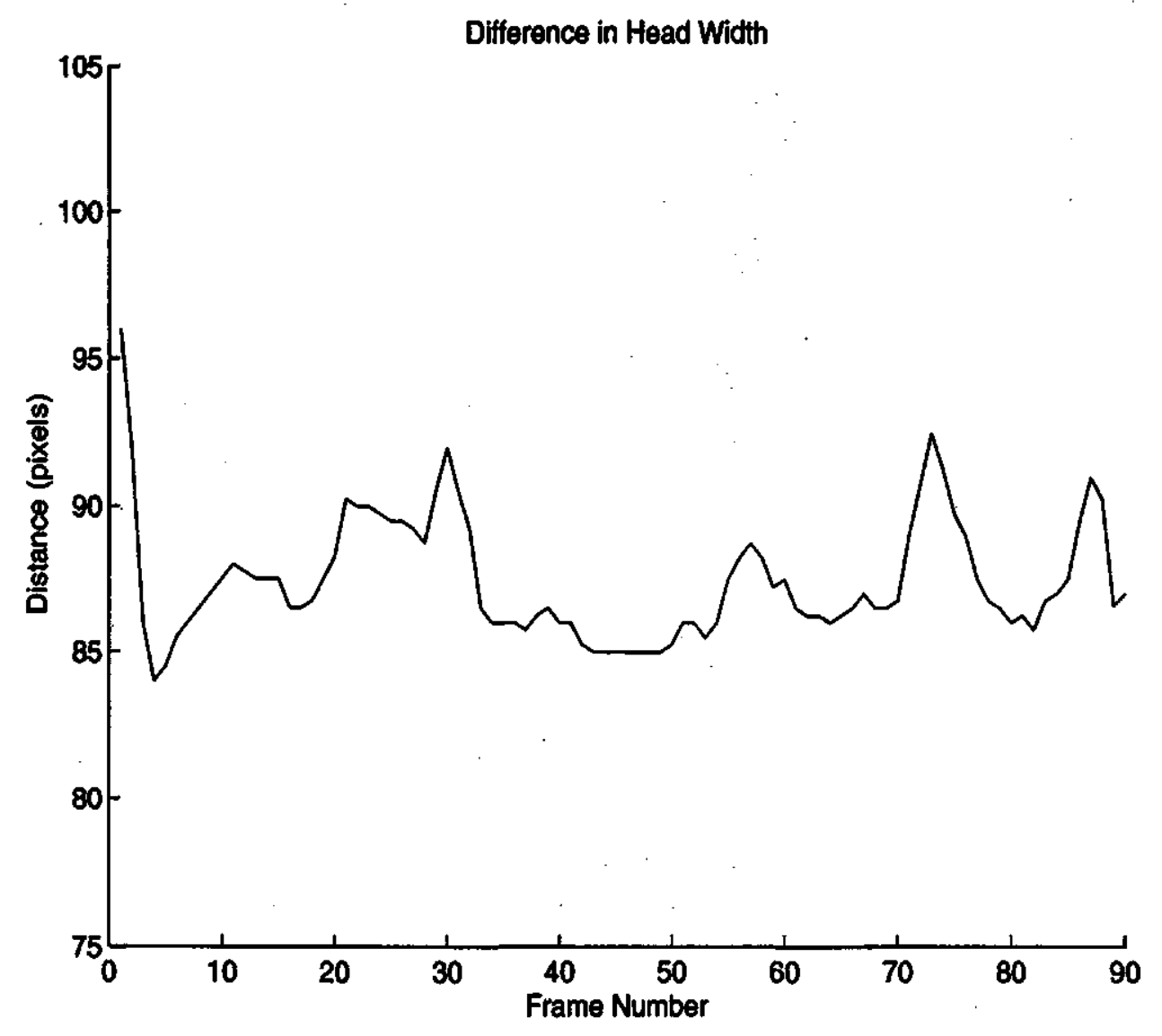

Figure 6.39 Width of the Head 


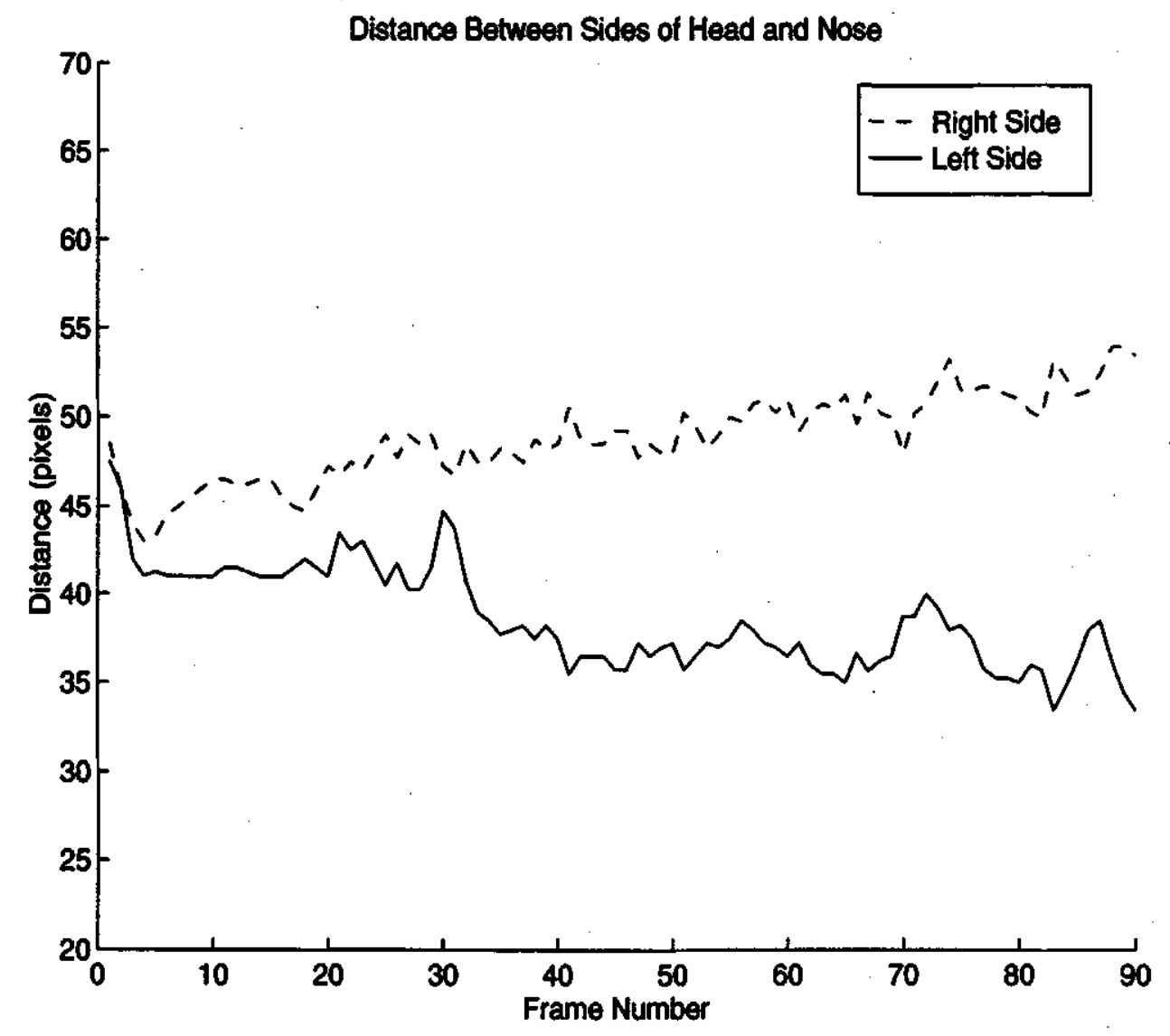

Figure 6.40 Side of Head to Nose Widths 
The Y-Z motion is best shown in vertical distances between the nose and other objects in the face. Ideally, the chin and forehead would make the best choice, however the chin could not be extracted. The chin is a very sensitive part of the face because the neck and chin tend to be similarly shaded. Therefore, corresponding encoder results to nonlinear scaling will not be possible. Instead mouth, eyes, and forehead positions will be selected to verify Y-Z movement in the face. It was suggested in chapter 5 that distances between these features should increase or decrease in y-coordinate displacement for Y-Z rotation. The maximum distance between the objects occurs when the face points directly at the camera. If this is true, similar movements should be noticed in all three graphs.

Fig. 6.41 is the variation of the distance between the nose and mouth. Fig. 6.42 shows the distance between the nose and the middle of the two eyes. Lastly, Fig. 6.43 is the distance between the forehead and the nose for the video sequence. In the graphs, the objects furthest from the nose show the most movement, which is best for Y-Z detection. The mouth would be a poor choice due to its proximity to the nose. The forehead is a much better choice. 


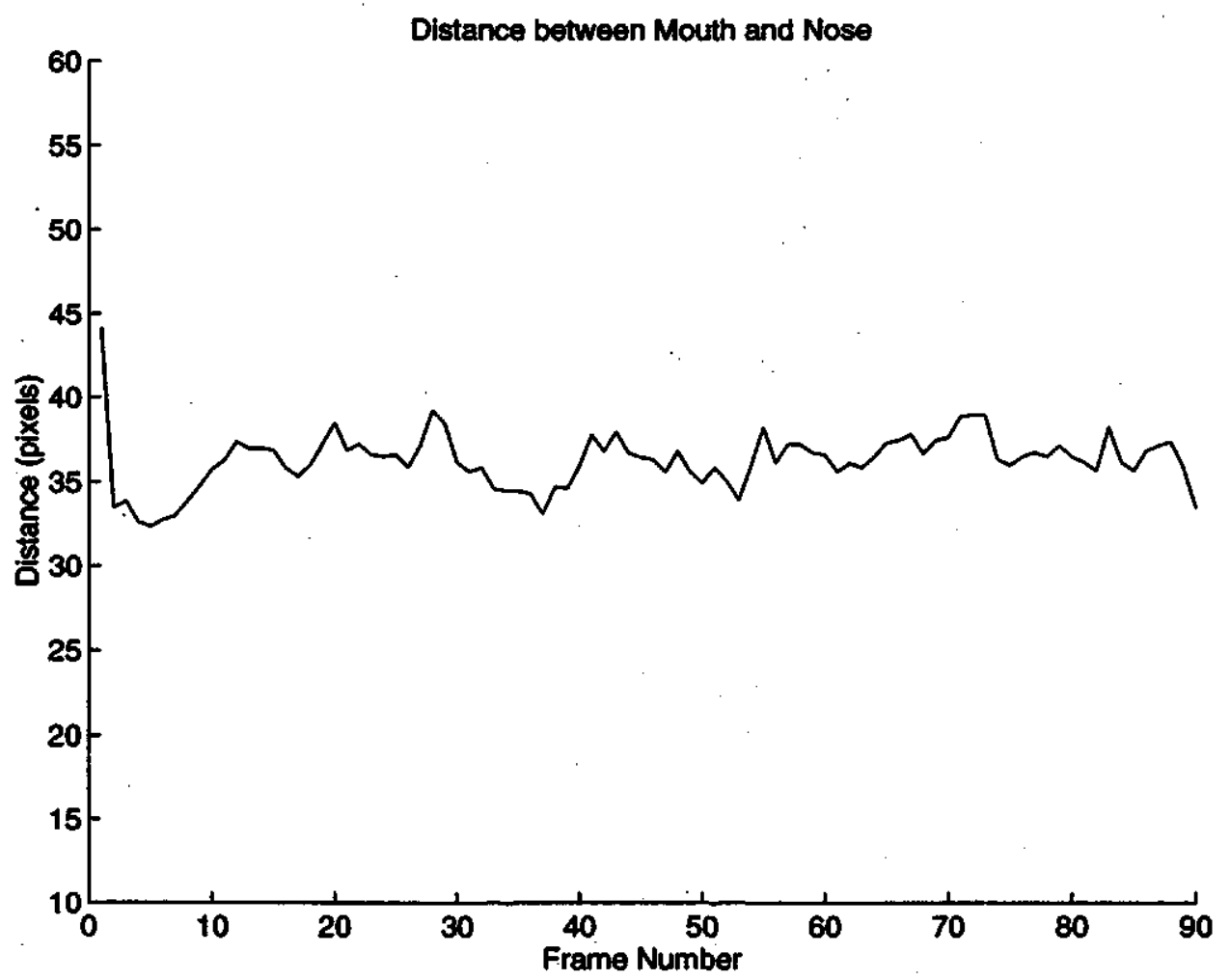

Figure 6.41 Variation of the distance between Nose and Mouth 


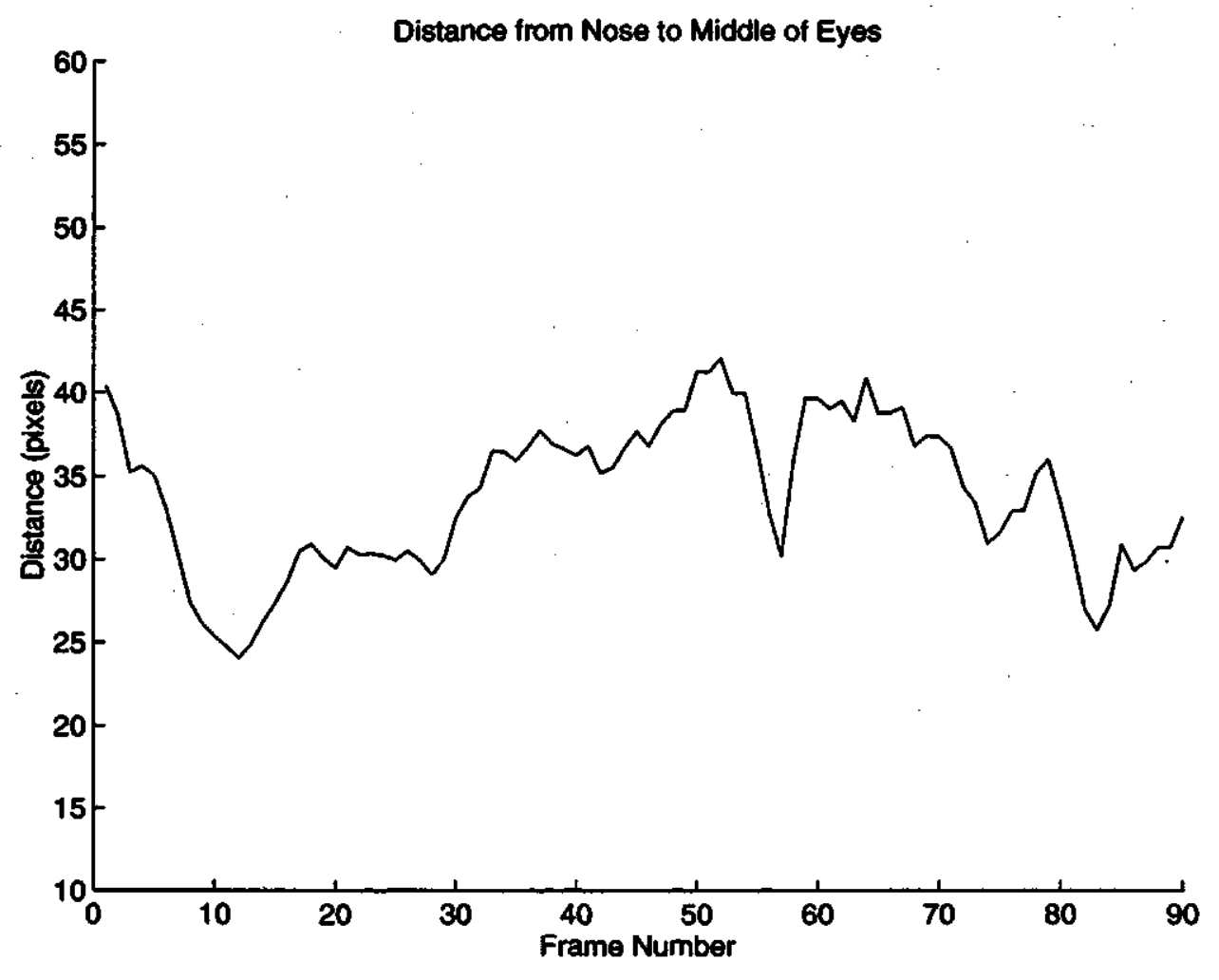

Figure 6.42 Variation of the distance between Nose to Middle of Two Eyes 


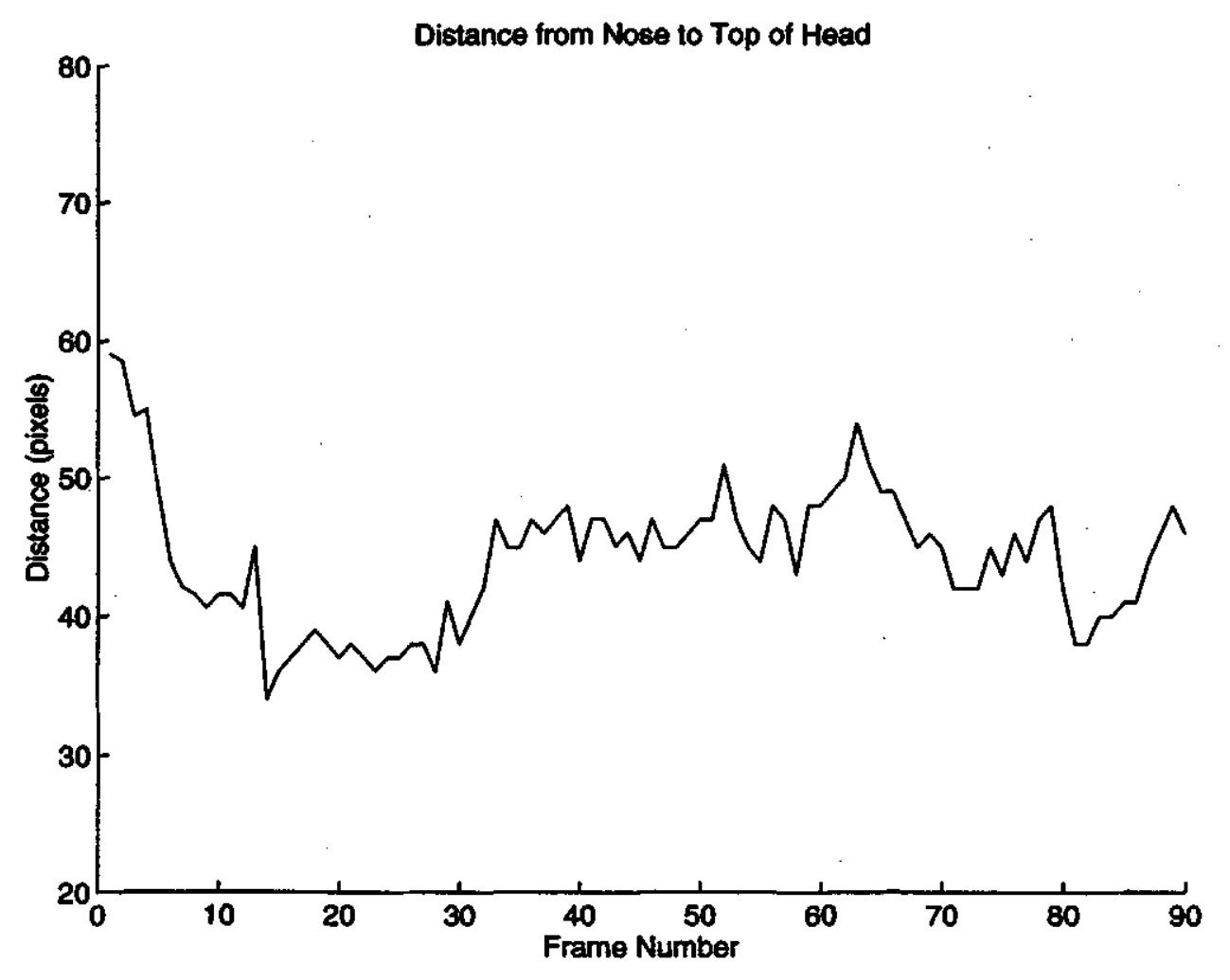

Figure 6.43 Variation of the distance between Nose and Forehead 


\subsubsection{Pattern Recognition and Extraction of X-Y Motion}

Pattern matching techniques are very different from the encoder measurement previously made. X-Y motion is found with respect to the outside edge of the head. The template for measuring movement is the edge in frame one of the database. The template is kept the same through out the 90 frame sequence because movement of outer edge information between adjacent frames is small.

The first test of X-Y motions was to determine if rotation of the head could be found. Displacement and scaling are not measured in this test since these measurements are almost negligible. To find the angle of rotation the choice of pivot is important, by observation the choice of pivot was located at the middle of the neck. Fig. 6.44 shows the angle of rotation of the extracted outside edge. The graph indicates a counterclockwise rotation of the head occurred over the 90 frame sequence. Objectively, the rotation angles looks larger than expected, but the observed template match was very close. The draw back of using pattern matching is the selection of pivot and the step size used to measure rotation, which is $0.29^{\circ}$ per interval. 


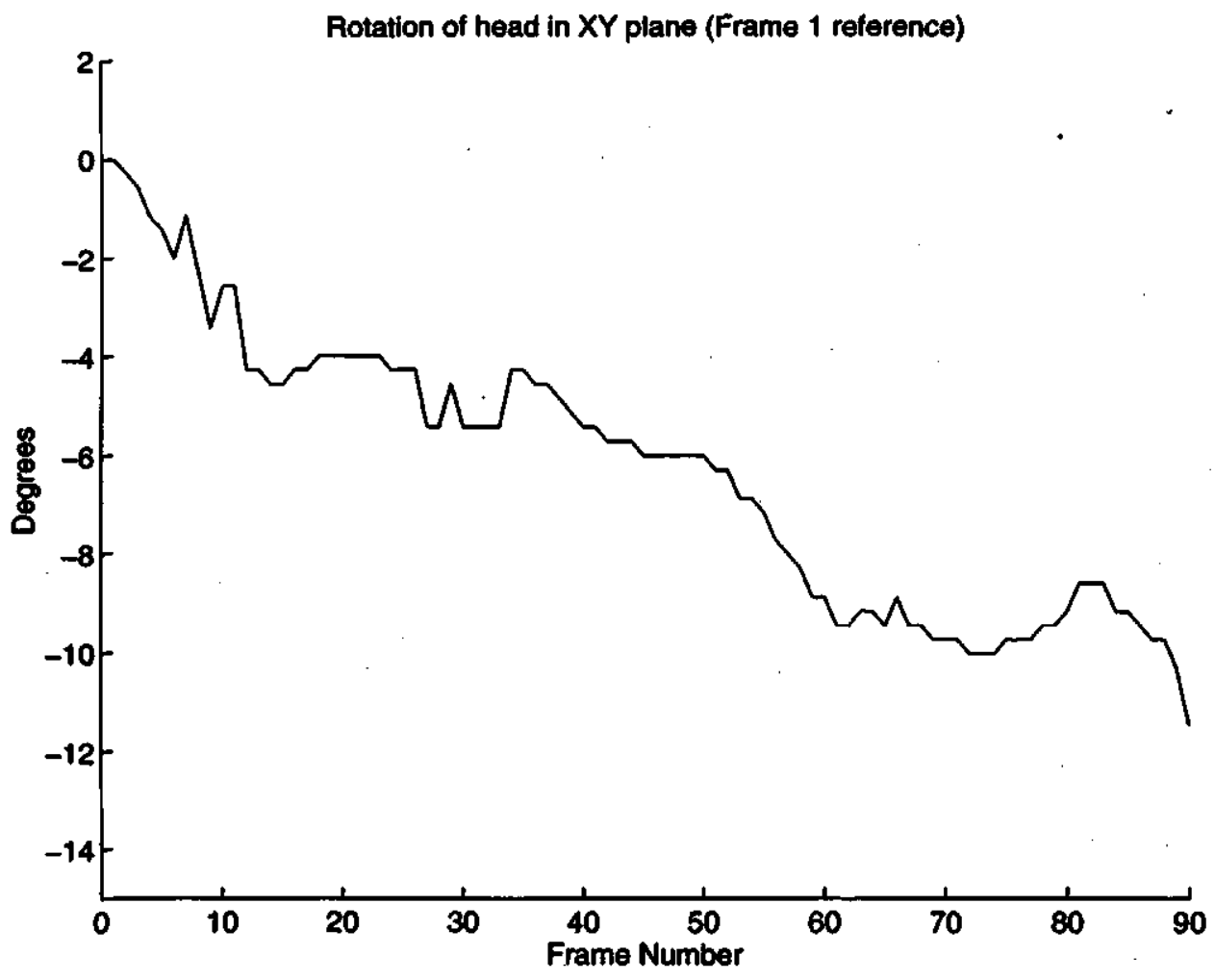

Figure 6.44 X-Y Rotation Measurements

Head rotation usually accompanies horizontal and/or vertical head displacement, which obscures the measurement of head rotation. To achieve the best possible match all template movements must be accounted for. The order of template movement will make a difference in the speed of the match. The two motions considered for determining order are displacement and rotation. In the database, the rotation of the head accounts for the majority of the motion.

The case in which displacement is applied before head rotation is shown in Fig. 6.45(a). The graph illustrates that the best match occurs when the displacement attempts to track the direction of rotation. In Fig. 6.45(b) displacement of the pattern was found after head rotation, little movement in the pattern was necessary to find the best fit. In comparing the two graphs, the displacement would be faster being matched last. The tests indicate that the order should favor the action with the most movement. In this case, rotation must be matched first. However, the favored action is unknown until the pattern matching method is applied. The decision to select the order must be decided by other means. 


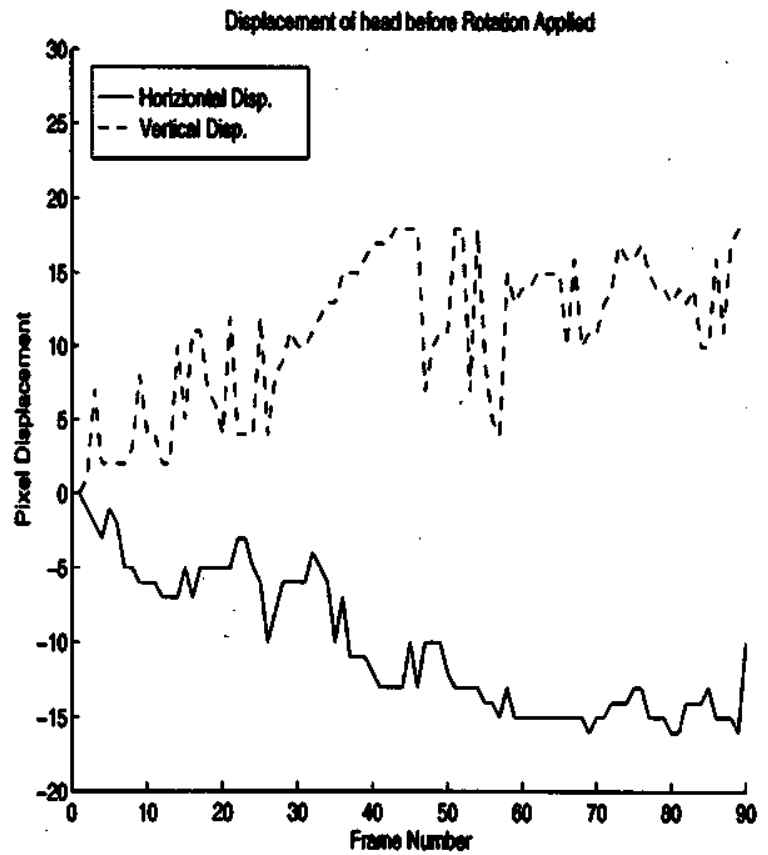

(a)

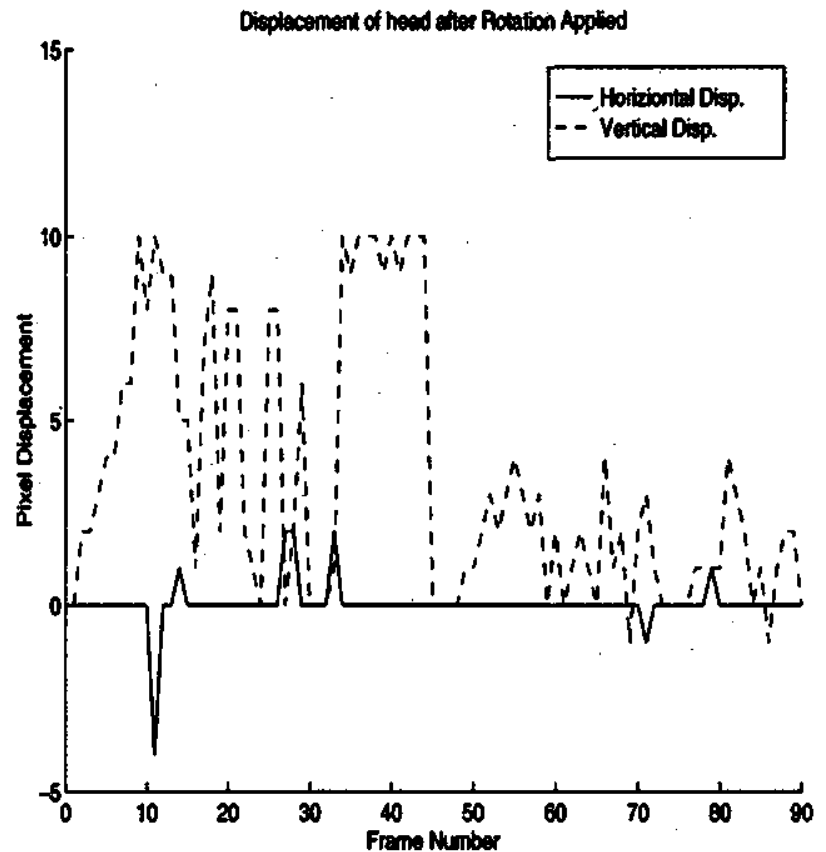

(b)

Figure 6.45 X-Y Displacement (a) before and (b) after Rotation 


\subsection{Volume of Data For Transmission}

In implementing the encoding and decoding algorithms in a video telephone, the amount of data to be transmitted over a telephone channel must be restricted. Use of encoding and decoding algorithms in this thesis require image data and measured parameters to follow a predefined format. This is the division of data into overhead and individual frame information.

\subsubsection{Overhead Information}

Overhead information is data that must be sent to the decoder upon initial startup of the system. This data includes the reference image of the person and initial grid. The section of data will introduce a delay in the operation of the system, but will only occur at the start.

The speed of transmission of the reference image depends on the size of the image and the use of image compression. Two common sizes are NTSC $(352 \times 240)$, and CIF (352×288). The ability of image compression depends on the method, but it is known that Huffman coding, and pixel difference can compresses to one fifth of the original volume. However, the use of image compression has a delay of its own. To minimize the delay would require special hardware. The estimation of time required for transmission of overhead data will consider image data uncompressed.

The mesh transmitted should be specific for three boundaries, the backgroundclosed boundary, the head boundary, and the face boundary. Each boundary is important in the fitting of the grid to the reference frame. The background-closed boundary is represented by grid nodes at the perimeter of the picture. Head boundary measurements are grid nodes around the head. Face boundary is the boundaries between the mouth and eyes. For estimating the size of data, the image is $252 \times 288$ pixels ( 8 bits per pixel) covered by a $26 \times 20$ grid. 


\subsubsection{Individual Frame Information}

Individual frame information is structured for the decoding algorithms described in chapter 4. The information is divided into object location, and decoding parameters.

Object location is the information on the positions of the nose, eyes, mouth, and head. The nose is a reference for the relative positions of the eyes, mouth, and face. The eyes and mouth data represent the relative position of the nose to the upper right point, and lower left point in each object. For the head, values represent the distance between the nose and the sides of the head block.

The decoder algorithms however require face motion to be described in simple parameters derived from measurements. $\mathrm{X}-\mathrm{Y}$ movement represents rotation, displacement, and scaling. Rotation needs a pivot $\left(x_{c}, y_{c}\right)$, and the angle of rotation $\theta$. Displacement requires two offset values $\left(d_{x}, d_{y}\right)$. Scaling uses two parameters, center of zooming $\left(x_{z}, y_{z}\right)$, and gain $a_{z}$. For the Attractor/Repellor, the eyes will only move in one direction, and the mouth two directions. The directions are define by $\alpha$ and $\beta$ for horizontal and vertical movement. For X-Z and Y-Z rotation, each plane of rotation requires the definition of the head boundaries and a reference node for motion. This information can be specified in the object location data. The amount of movement is controlled by the terms $a_{x}$ for X-Z, and $a_{y}$ for $\mathrm{Y}-\mathrm{Z}$. 
OverHead Data

Reference Image $(352 \times 288)$

101,376 bytes

Mesh Information $(26 \mathrm{w} \times 20 \mathrm{~h})$

520 integers

Boundary Information $3 \times 2 \times(26 \mathrm{w}+20 \mathrm{~h}) \max$

276 bytes

Total OverHead

102,692 bytes

Total Transmission time (33.6 kbaud)

24.6 seconds

Individual Frame Data

Object Location

Nose Position

2 integers

Eyes Object Position

$2 \times 4$ integers

Mouth Object Position

4 integers

Face Position

4 integers

Decoder Mesh Parameters

Scaling : $\left(x_{c}, y_{c}\right)$ and $a_{z}$

2 integers

1 float

Displacement : $\left(d_{x}, d_{y}\right)$

2 integers

Rotation : $\left(x_{z}, y_{z}\right)$ and $\theta$

2 integers

1 float

X-Z Rotation : $a_{x}$

1 float

Y-Z Rotation : $a_{y}$

1 float

Attractor Repellor

Eyes : 2 mass points and $\alpha$

$2 \times 3$ integers

Mouth: 4 mass points and $\alpha$ and $\beta$

6 integers

Total frame Data 88 bytes

Total Transmission rate at $30 \mathrm{fps}$

21.1 kbaud 
To transmit facial coding it must operate on telephone modem technology of $33.6 \mathrm{kbaud}$. The success of the coding scheme depends on the individual frame transmission being less than the maximum data rate. The estimate shows this is true with a transmission rate of $21.1 \mathrm{kbaud}$. The overhead data is only used at start of the transmission. To a user of a video telephone, an initial startup delay of 25 seconds would be unacceptable; therefore, image compression of the image is necessary. 


\section{Conclusion}

\subsection{Conclusion}

Video image compression techniques such as MPEG can achieve a reasonable compression to make transmission of the compressed data through a low bit-rate channel. Any further reductions in the bit-rate require descriptive information specific to a type of image, say facial images, to be extracted from the source image and to be transmitted as highly condensed information instead of the physical image. This approach is followed in this thesis and facial images are targeted because of the potential applications to video telephone and conferencing. The term "descriptive" can be linguistic terms such as "laughing" or "crying" or can be numerical as " a mouth opening of $\frac{1}{4}$ inch ". It is evident that the encoder must be intelligent supported by a knowledge base.

In order to realize such video image compression, the decoder must produce a simulated image since only abstract information describes the present state of a face. This simulated image is produced by warping a given facial image prior to the transmission of a series of images.

The low bit-rate video image compression is comprised of a encoder and a decoder, which must work harmoniously under common specifications. However, the key roles of the encoder is to extract the features important for image warping. The key roles of the decoder is to efficiently warp the given image.

In image warping, an image can only be warped by its frame where the details of the image is attached. This frame is referred to as a mesh. The manipulations necessary for warping are controlled by the mesh. Hence, it is crucial to set up a most suitable mesh for the warping algorithm used. 
In this conclusion, any significant experimental findings and new developments made in terms of algorithms and computer programs are summarized for each of the following key issues dealt in the thesis

(1) Image Warping Techniques

(2) Mesh Manipulation Techniques

(3) Facial Feature Detection Techniques

\section{Image Warping Techniques}

The mesh considered in this thesis was derived from the two separate theories on image warping. The two methods of warping are bilinear interpolation, and cubic spline interpolation. The spline is pixel manipulation scan line by scan line with the help of curve fitting, resulting in high quality warping. The bilinear interpolation method is based on the fitting of pixel data from a source rectangle to its destination rectangle by using the linear interpolation of $x$ and $y$-coordinates of the source rectangle, individually. Quality of the image is sacrificed in this method to achieve speed and grid flexibility. The speed is favored in the designed low bit-rate image coding system.

\section{Mesh Manipulation Techniques}

It was learned in mesh development that the simplicity of the mesh was important in the performance of the decoder. Preliminary work on grid structures used a complex grid, or more accurately a mask. The mask proved to be very flexible in simulating motion by using action units. However, the detailed mask requires accurate detection of the landmarks set on each of the facial parts, where as a rectangular mesh was manipulated by more crude feature information. The testing of each mesh algorithm for the rectangular grid proved to be a more effective in simulating facial motion than mask approach. The use of the rectangular grid is a better solution because it is much easier to control the mesh with these mesh algorithms rather than using action units. 
The rectangular mesh is expected to easily open and close the eyes and mouth, and rotate the head in three dimensions. The Attractor/Repellor technique was created to perform an opening and closing action. The range of motion is controlled by the variable $\mathrm{n}$. The tests showed that the useful range of grid motion requires $n$ between 1.0 and 2.5. The choice of $n$ depends on how spreading in the mesh should be localized and how evenly or unevenly grid lines should be spread. To control exact movement the gain factors $\alpha$ and $\beta$ are also needed. For direction of motion, two mass nodes per direction are seemingly best. More complex actions would require more mass nodes.

$\mathrm{X}-\mathrm{Y}$ motion for the head was easily realized since it is based on simple displacement, rotation, and scaling of grid nodes. Effective simulation of this type of movements is limited by the perspective of the reference frame and choice of the head's grid. For a picture of a person looking straight at the camera a movement of $-4^{\circ}$ to $+4^{\circ}$ looks natural. Beyond this range distortion is observable in the picture due to the rectangular shape of the grid.

Both X-Z and Y-Z rotation are based on the three-point approach for simulating motion. The outer edges of a face and an inner reference point can be used for unevenly separating the grid. Two parameters responsible for the motion, $a$ and $n$, control the motion of the inner reference and the degree of nonlinearity. $a$ is derived from the position of the reference point and is the main control for the decoding action. For a certain value of $n$, a wrap around effect occurs in the simulated rotation. Overlapping which was undesirable in the $A / R$ now benefits in rotation. However, the perspective of the person in the reference frame limits the degree of motion.

\section{Facial Feature Detection Techniques}

The objective of the encoder is to measure the parameters required for the decoder. The work done for the encoder was to prove whether extraction of such parameters from an image is possible. Information collected was able to detect eye and mouth movement, and head rotation. The methods of encoding for the eyes and mouth directly use measurements of the objects in the face. Head rotation is however inter- 
preted from the interframe measurements.

Eye and mouth measurements are specifically width and length measurements. The only motion considered by these measurements is limited to the two directions that satisfy the requirements of the $A / R$.

$\mathrm{X}-\mathrm{Y}$ head rotation measurement uses the method of pattern recognition. The pattern chosen is the outside of the head. By determining the best fit between two frames, the amount of image displacement and rotation was found.

$\mathrm{X}-\mathrm{Z}$ motion requires three points from the extracted head to keep track of X-Y motion. The best choice of points for deciding rotation is using a point on each side of the face, and the nose as the reference for direction of motion. Extraction of this information is always successful. Therefore, carrying out $\mathrm{X}-\mathrm{Z}$ motion is straightforward.

Y-Z motion uses the same principle as X-Z motion, but due to the inability to extract chin movement tests could not be compared to decoder requirements. Nonlinear scaling would have required the forehead, chin, and the nose as a reference. However, tests were conducted on the mouth, a point between the eyes, and the forehead with the nose as a reference. The movement of the points verified the need for a nonlinear function to simulate rotation.

The parameters discussed for each method of decoder motion are not the only parameters needed for describing facial motion of objects. Requirement for a low-bit rate facial image coding scheme must consider all possible parameters. The code structure requires that two distinct data types, overhead information and individual frame information. Overhead information is not sent in real-time because it contains image information, and the reference grid. Sending a standard NTSC picture or a CIF picture with the grid would take up to 30 seconds to transmission. Individual frame information includes the decoder parameter plus information on location of facial objects. At a 30 fps transmission frame information is equivalent to 21 kbaud. Therefore, this low bit-rate application is able to transmission over a telephone line. 


\subsection{Future Research}

There are many possibilities available in the further development of this project, as well other possible applications for this technology. The main interest is in the achievement of a real-time system, which is a transmission of images at $30 \mathrm{fps}$.

The work in developing the decoder and encoder has not been taken to completion. For a complete system, the encoder measurements must be explicitly converted in terms of the requirements of the decoder. This not a problem since the encoder and decoder work under the same principle to derive motion. The validity of the encoder information should be verified by producing a series of simulated video sequences. The comparison of the simulated sequence to the real sequence would provide a better feel for the capability of the method.

To improve the head rotation algorithms the rectangular head block must be fitted to the outside edge of the head. By doing this head simulation may appear more realistic because warping will be constrain to the person not the background. To apply the warping algorithm must use flexible polygon grids rather than rectangular ones.

To verify the system as operating as a low bit-rate image transmission system, a system using two computers one acting as the encoder, and the other the decoder needs to be developed. Algorithms (partially developed but not reported in this thesis) should be developed to pass information between the two systems via a modem or serial port connection. The coded structure of data that includes decoder information, and object location information must be also verified using such a system.

To reach the goal of a real-time system the work must go on in the development of special hardware. With the availability of moderately priced fast DSP microprocessors the development of a system that can meet the specifications is possible. The algorithms of image warping and edge detection are slow because of the repetitive multiplication and additions. This will be alleviated by a DSP processor. 
The encoder used in this thesis was considered as very basic. To improve the encoder some intelligence must be incorporated to give the computer greater understanding of the structure in the face. Object recognition and neural networks may offer such required intelligence. These methods have been proven very useful for applications in the area of pattern recognition.

Other future research could involve the use of voice recognition as a method for simultaneous voice transmissions so that applications such as voice mail can enjoy motion pictures of the person talking while receiving the message. 


\section{References}

[1] Rick Whiting, "Max Thruput on Dial-up Lines", Post to USENET Newsgroup comp.dcom.telecom, Internet Web Page: http://ww .dnai.com/ winske /maxthruput.txt, Jan 1996.

[2] "Wavelets offer better-than-JPEG compress at low cost", Computer Design, Jan. 1996: 63.

[3] Paul Ekman and Wallace.V.Friesen, Facial Action Coding System, Palo Alto, California: Consulting Psychologists Press Inc, 1978.

[4] Chang Choi,Kiyoharu Aizawa, Hiroshi Harashima, and Tsuyoshi Takebe, "Analysis and Synthesis of Facial Image Sequences in Model Based Image Coding", IEEE Transactions on Circuits and Systems for Video Technology, Vol 4, No. 3, June 1994.

[5] George Wolberg, Digital Image Warping, New York: IEEE Computer Society Press Monograph, 1990.

[6] William.K.Pratt, Digital Image Processing, 2nd ed., Toronto: John Wiley \& Sons Inc, 1991.

[7] Kevin.L.Gong and Lawrence.A.Rowe, "Parallel MPEG-1 Video Encoding", Computer Science Division-EECS, University of California, Berkeley, CA, May 1994.

[8] P.Ekman and E.Scherer, Approaches to Emotion, HillsDale, N.J: L.Erlbaum and Associates, 1984.

[9] Paul Ekman, Emotions of the Human Face, New York: Cambridge University Press, 1982. 
[10] Whoi-Yul Kim, Poras.T.Balsara, David.T.Harper, Jon Wong Park, "Hierarchy Embedded Differential Images for Progressive Transmission Using Lossless Compression, IEEE Transactions on Circuits and Systems for Video Technology, Vol 5, No. 1, 1995.

[11] Kunio Takaya, Charbel G. Tannous and Li Yuan, "Information Preserved Guided Scan Pixel Difference Coding for Medical Images", IEEE Wescanex 95 Conference,Communications, Power and Computing Proceedings, pp. 238-243, Vol 1, Winnipeg, Manitoba, 1995.

[12] Haibo $\mathrm{Li}$ and Rober Forchheimer, "Two-View Facial Movement Estimation", IEEE Transactions on Circuits and Systems for Video Technology, Vol 4, No. 3, 1994.

[13] Gary Faigin, The Artists Complete Guide to Facial Expressions, New York: Watson-Guptill Publications, 1990.

[14] J.N.Reddy, An Introduction to Finite Element method, New York: McGraw Hill, 1984.

[15] Arun.N.Netravali and Barry.G.Haskell, Digital Pictures Representation and Compression, New York: Plenium Press, 1988.

[16] Harley.R.Myler and Arthur.R.Weeks, The Pocket Handbook of Image Processing Algorithms in C, New Jersey: Prentice Hall, 1993.

[17] Harley.R.Myler and Arthur.R.Weeks, Computer Imaging Recipes in C, New Jersey: Prentice Hall, 1993.

[18] William.H.Press., et al.Numerical Recipes and C, 2nd ed., New York: Cambridge University press, 1992.

[19] Matlab: High Preformance Numeric computation and Visualization Software (users guide), Natick, MA: The MathWorks Inc, 1992. 
[20] Matlab: High Preformance Numeric computation and Visualization Software (reference guide), Natick, MA: The MathWorks Inc, 1992.

[21] Herbert Schildt, Using Turbo C, 2nd ed., Toronto: McGraw Hill, 1989.

[22] Jeffrey Weiss and Doug Schremp, "Putting Data on a Diet", IEEE Spectrum, August, 1993, pp. 36-39.

[23] "An Abundance of Video Format", IEEE SPECTRUM, Mar. 1992: 26-28.

[24] Summit Basu,Irfan Essa,Alex Pentland, "Motion Regularization for Model-Based Head Tracking", Perceptual Computing Section, The Media Laboratory, Massachetts Institute of Technology, Cambridge, MA, Jan. 1996.

[25] J.Spanier and K.Oldham, An Atlas of Functions, Washington: Hemisphere Pub. Corp, 1987.

[26] Quiring and Warfel, Head, Neck, and Trunk, Philidelphia: Lea \& Febiger, 1960.

[27] Leslie Lamport, LTEX User's Guide and Reference Manual, 2nd ed., Don Mills, ON: Addison-Wesley Publishing Company, 1994.

[28] Mark Nelson, The Data Compression Book, California: M \& T Books, 1992.

[29] Alvin.L.Liff, Color and Black and White Television Theory and Service, 2nd ed., Prentice Hall: New Jersey, 1985. 


\section{A. Software Algorithms}

This appendix contains brief description of the structure for each program contained in the diskette enclosed.

\section{FITTING ACTION UNIT MASK TO AN IMAGE: tri_fit.cpp}

INPUT: Base mesh.

1. Fit base mesh to image of "Todd" by sizing width and length.

2. Fit each of the following parts the same way as the head, left eyebrow, left eye, right eyebrow, right eye, nose, and mouth.

3. Check for overlaps.

4. Save resulting mesh.

ACTION UNIT SETTING PROGRAM: tri_au.cpp

INPUT: Fitted mask, triangle placement information.

1. Load input information.

2. Select Action Unit.

3. Select iterations.

4. Select direction.

6. Apply AU to mask using iterations, direction.

7. View each iteration and save the resulting mesh. 
INPUT:Source image,

Source, and Destination masks.

Triangle information.

1. Warp image with bilinear interpolation.

2. View results.

3. Save new image.

\section{CUBIC SPLINE WARP :cubic.cpp}

INPUT:Source Grid and Image

Destination Grid

Grid and Image Dimensions $T$ and $I n$

1. Do horizontal spline fitting of grid to create tables $T_{i}$ and $T_{s}$ of dimensions $T_{w} \times I n_{h}$.

2. Use the tables to warp each horizontal line in the source image and correct the image by resample_gen.

3. Do vertical spline fitting of the grid to create tables $T_{i}$ and $T_{d}$ of dimensions $I n_{w} \times T_{h}$.

4. Use the tables to warp and correct each vertical line in the image created by step 2 . 
DECODER : decoder.cpp

INPUT:Parameters for $A / R, X-Y$ rotation,

$\mathrm{X}-\mathrm{Z}$ rotation, Y-Z rotation and displacement.

1. Load Parameters.

2. Apply A/R parameters to the eyes.

3. Apply $A / R$ parameters to the mouth.

4. Rotate head in X-Y plane.

5. Rotate head in X-Z plane.

6. Rotate head in Y-Z plane.

7. Displace the head in $X$ and $Y$ directions.

8. Apply warping algorithm (Bilinear interpolation contained in this program). 
INPUT: Sequence of images of 'Claire'.

1. Sequence is repeated with frame 1 used as a reference for motion.

2. Load image.

3. Apply Average Amplitude Projection to find the head block.

4. Resize image to the size of the head block.

5. Find the average pixel of the image for the threshold filter.

6. Apply four gradient filters NE, SE, SW, NW.

7. Combine the filter's results.

8. Apply four more gradient filters N,S,E,W.

9. Combine these four filter's results.

10. Combine all eight filter results, then apply the threshold filter.

11. Apply Laplacian filter, if Gradient not used.

12. Extract singularities in the detected edges.

13. Extract outside edge of the head.

14. If the current frame is not 1 then find $X-Y$ motion (displacement and rotation) using pattern recognition.

15. Sweep the inside of the face to find facial objects.

16. If on frame 1 save objects else compare the objects to the objects in the previous frame. Then save the objects.

17. Find the mouth objects by using the reference frame mouth position or by the position of the nose. This object position will be used to resample pixel values around it and use a threshold filter to find a better edge of the mouth.

18. Measure the width and length of the mouth.

19. Find the eye objects by comparing positions from previous frames, or find it using the relative position to the nose.

20. Measure the widths and lengths of the eyes.

21. Find sides of the face accross from the nose, and the forehead. 